MIT-CTP-3726

UCSD/PTH 06-04

hep-ph/0605001

\title{
The Zero-Bin and Mode Factorization in Quantum Field Theory
}

\author{
Aneesh V. Manohar ${ }^{1}$ and Iain W. Stewart ${ }^{2}$ \\ ${ }^{1}$ Department of Physics, University of California at San Diego, La Jolla, CA 92093* \\ ${ }^{2}$ Center for Theoretical Physics, Laboratory for Nuclear Science, Massachusetts \\ Institute of Technology, Cambridge, MA 02139†
}

\begin{abstract}
We study a Lagrangian formalism that avoids double counting in effective field theories where distinct fields are used to describe different infrared momentum regions for the same particle. The formalism leads to extra subtractions in certain diagrams and to a new way of thinking about factorization of modes in quantum field theory. In non-relativistic field theories, the subtractions remove unphysical pinch singularities in box type diagrams, and give a derivation of the known pull-up mechanism between soft and ultrasoft fields which is required by the renormalization group evolution. In a field theory for energetic particles, the soft-collinear effective theory (SCET), the subtractions allow the theory to be defined with different infrared and ultraviolet regulators, remove double counting between soft, ultrasoft, and collinear modes, and give results which reproduce the infrared divergences of the full theory. Our analysis shows that convolution divergences in factorization formulæ occur due to an overlap of momentum regions. We propose a method that avoids this double counting, which helps to resolve a long standing puzzle with singularities in collinear factorization in QCD. The analysis gives evidence for a factorization in rapidity space in exclusive decays.
\end{abstract}

\footnotetext{
* Electronic address: amanohar@ucsd.edu

$\dagger$ Electronic address: iains@mit.edu
} 


\section{Contents}

I. Introduction 3

II. NRQCD and SCET 7

A. Comparison with the NRQCD Method of Regions 7

B. Summing Logarithms 11

III. Infrared Modes in NRQCD and SCET 12

IV. Zero-Bin Subtractions 17

V. Zero-bin Subtractions in NRQCD (Non-relativistic Processes) 21

A. NRQCD Soft Crossed-Box Graph 21

B. NRQCD Box Graph 24

C. Results for the Box and Crossed-Box at order $\mathcal{O}\left(v^{2}\right) \quad 26$

VI. Zero-bin Subtractions in SCET $_{I}$ (Inclusive Processes) 29

A. Onshell Integrals and a Cutoff Regulator for $B \rightarrow X_{s} \gamma$

B. Offshell Regulator with Dimensional Regularization for $B \rightarrow X_{s} \gamma$

C. Production of $n-\bar{n}$ jets 35

D. $\gamma^{*} \rightarrow q \bar{q} g$ : The Zero-bin at Tree Level and for Phase Space Integrals 37

VII. Zero-bin Subtractions in SCET $_{\text {II }}$ (Exclusive Processes) 42

A. A SCET II Loop Integral with Subtractions 46

1. Soft-Collinear Division with a Hard Cutoff Regulator 50

B. Dimensional Regularization Division in $\mathrm{SCET}_{\mathrm{II}}$ : General Discussion 53

1. Dimensional Regularization for the one-loop example 56

C. Zero-Bin Subtractions in Convolutions: General Discussion 60

D. The $\rho-\pi$ Form Factor at Large $Q^{2} \quad 66$

E. Factorization of $\zeta^{B \pi}(E)$ appearing in $B \rightarrow \pi \ell \bar{\nu}$ and $B \rightarrow \pi \pi$

VIII. Conclusion 76

A. Rapidity Cutoff Loop Integrals in $\mathrm{SCET}_{\mathrm{II}}$

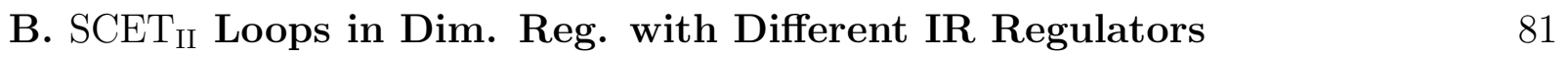

i) Three IR Masses, $\mathbf{m}_{\mathbf{1}}, \mathbf{m}_{\mathbf{2}}, \mathbf{m}_{\mathbf{3}}$

ii) Offshellness $\mathbf{p}^{\mathbf{2}}=-\mathbf{P}^{\mathbf{2}} \neq \mathbf{0}, \ell^{\mathbf{2}}=-\mathbf{L}^{\mathbf{2}} \neq \mathbf{0}$, with $\mathbf{m}_{\mathbf{1}} \neq \mathbf{0}$ and $\mathbf{m}_{\mathbf{2}, \mathbf{3}}=\mathbf{0}$

iii) The limit $\mathbf{m}_{\mathbf{1}} \rightarrow \mathbf{0}$ of cases i) and ii) 85

References 


\section{INTRODUCTION}

Many problems of interest in quantum field theory have several momentum scales, and are efficiently treated using effective field theory (EFT) methods. One constructs a sequence of effective field theories which focus on one scale at a time. This greatly simplifies the calculations, partly because new symmetries emerge, and partly because Feynman graphs in each effective theory are much simpler to evaluate than the multiscale integrals of the full theory. More recently, theoretical methods have been developed which allow one to analyze field theories with several small momentum scales which are coupled by the dynamics. In these theories, it becomes necessary to treat the coupled momentum scales simultaneously within a single effective theory, rather than sequentially in a series of several different effective theories. Examples of theories with coupled scales are the soft-collinear effective theory (SCET) for energetic particles [1, 2, 3, 4], and any non-relativistic theory where the kinetic energy is a relevant operator, examples being non-relativistic QED (NRQED) [5] and nonrelativistic QCD (NRQCD) [6, 7, 8, 9, 10].

We wish to discuss an issue in the separation of infrared (IR) regions which appears at first to be a technical subtlety, but turns out to have important physical ramifications. It results in a tiling theorem for IR modes in quantum field theory. In the examples we discuss, it has to do with the proper treatment of soft modes in NRQCD/NRQED and of collinear and soft modes in SCET. In NRQED, the photon field of the fundamental QED theory is replaced by two fields, describing soft and ultrasoft (usoft) photons with energies of order $m v$ and $m v^{2}$ respectively, where $v \ll 1$ is the typical fermion velocity in the non-relativistic bound state. The soft and usoft NRQED gauge fields are $A_{p}^{\mu}(x)$ and $A^{\mu}(x)$, where $p$ is a label momentum of order $m v$, and $k$, the Fourier transform of $x$, is of order $m v^{2}$ [8]. The two fields describe photons with momenta $p+k$ and $k$, respectively. In the special case that $p=0$ (the zero-bin), the soft photon becomes ultrasoft, and there is a double-counting of modes. To avoid double counting, the soft sector of the theory must have the additional constraint that $p \neq 0$. This paper explores the consequences of implementing zero-bin constraints for soft modes in NRQED/NRQCD, and the analog for collinear modes in SCET. In loop graphs, for example, the sum over soft intermediate states should be $\sum_{p \neq 0}$ rather than the conventional $\sum_{p}$. The difference, as we discuss in detail, is that conventional results have to be modified by zero-bin subtractions.

The zero-bin subtraction solves a number of problems in NRQCD and SCET, and also resolves the long standing puzzle of divergent convolutions in QCD factorization formulas. We discuss several applications:

1. Soft box graphs in NRQCD have unphysical pinch singularities in the energy integral,

$$
\int \frac{d k^{0}}{\left(k^{0}+i 0^{+}\right)\left(-k^{0}+i 0^{+}\right)} f\left(k^{0}\right),
$$


which make them ill-defined, even in dimensional regularization. In previous computations, it has been argued that these pinch singularities should be dropped in evaluating box graphs at any order in $v$ 9, 11, 12]. Pinch singularities are also a problem for the method of regions [13]. A direct application of the method of regions for $d^{4} k$ leads to ill-defined integrals, so it was defined to apply to NRQCD only after first doing the energy integrals. The zero-bin subtraction modifies the soft box graphs so that pinch singularities are absent, and the graphs are well defined.

2. The zero-bin subtraction automatically implements the previously studied pullup mechanism in NRQCD [14, 15], which was shown to be a necessary part of the definition of this type of theory with multiple overlapping low energy modes. Through the pullup, infrared (IR) divergences in soft diagrams are converted to ultraviolet (UV) divergences and contribute to anomalous dimensions.

3. There is a similar pullup mechanism at work in SCET for collinear diagrams. The anomalous dimensions of the SCET currents for endpoint $B \rightarrow X_{s} \gamma$ and $B \rightarrow X_{u} \ell \bar{\nu}$ were computed in Ref. [1, 2] from the $1 / \epsilon$ and $1 / \epsilon^{2}$ terms. Some of these terms in the collinear graphs are actually infrared divergences. The zero-bin subtraction converts these infrared divergences to ultraviolet divergences so that IR-logs in QCD can be resummed as UV-logs in the effective theory. This formally justifies the results used for anomalous dimensions in these computations, and in subsequent work for other processes with similar anomalous dimensions eg. [16, 17, 18, 19, 20, 21].

4. In high energy inclusive production such as $\gamma^{*} \rightarrow q \bar{q} g$, there is a potential double counting at the edges of the Dalitz plot in SCET, which is resolved by properly taking into account the zero-bin in both fully differential and partially integrated cross sections.

5. As a by-product of our analysis we give definitions for NRQCD and SCET that are independent of the UV and IR regulators. We also demonstrate a link between power counting and reproducing infrared divergences in the EFT. Exploiting this link, we demonstrate that a choice exists for the degrees of freedom in NRQCD and SCET which is complete, covering all infrared regions for a broad class of physical situations. For these cases no new modes are required at any order in $\alpha_{s}$ or in the power expansion.

6. In high energy exclusive production, such as $\gamma^{*} \rightarrow \pi \rho$ or $\gamma^{*} \rightarrow \pi \pi$, there are unphysical singularities in convolution integrals of some hard kernels with the light-cone wavefunctions $\phi_{\pi}(x)$. For example

$$
\int_{0}^{1} \mathrm{~d} x \frac{\phi_{\pi}(x)}{x^{2}},
$$


which is divergent at $x \rightarrow 0$ if $\phi_{\pi}(x)$ vanishes linearly as $x \rightarrow 0$. The same is true for exclusive light meson form factors at large $Q^{2}$, as well as processes like $B \rightarrow \pi \ell \bar{\nu}$ and $B \rightarrow \pi \pi$ for $E_{\pi} \gg \Lambda_{\mathrm{QCD}}$. The zero-bin subtraction removes the singularity, and induces a corresponding UV divergence. After renormalization we have a finite convolution with $\phi_{\pi}(x)$, and the kernel behaves as a distribution we call $\varnothing$ :

$$
\int_{0}^{1} \mathrm{~d} x \frac{\phi_{\pi}(x)}{x^{2}} \rightarrow \int_{0}^{1} \mathrm{~d} x \frac{\phi_{\pi}(x)}{\left(x^{2}\right)_{\varnothing}}=\int_{0}^{1} \mathrm{~d} x \frac{\phi_{\pi}(x)-\phi_{\pi}(0)-x \phi_{\pi}^{\prime}(0)}{x^{2}}+\tilde{\phi}_{\pi}<\infty .
$$

The $\tilde{\phi}_{\pi^{-}}$-type term involves a $\ln \left(E_{\pi}\right)$ and is induced by UV renormalization in rapidity space. These terms are discussed in the body of the paper. Thus using SCET finite amplitudes are obtained for apparently singular hard scattering kernels.

7. The zero-bin procedure gives insight into factorization formulas which separate modes in rapidity space rather than by scale separation in their invariant mass. For example, two hadrons both built of non-perturbative modes with $p^{2} \sim \Lambda_{\mathrm{QCD}}^{2}$ can have their modes factorize by being in different corners of phase space or rapidity. We discuss this by applying the zero-bin technique to the formulation of degrees of freedom in $\mathrm{SCET}_{\text {II }}$ with Wilson lines on the light-cone. The separation between soft and collinear regions is controlled by perturbation theory with dependence on a rapidity parameter.

Examples of processes where endpoint singularities in convolution integrals have been encountered include the pion form factor at large $Q^{2}$ and subleading twist [22], the $\rho-\pi$ form factor [23], the $B \rightarrow \pi \ell \bar{\nu}$ form factor [24], form factor terms in $B \rightarrow \pi \pi$ [25], the Pauli nucleon form factor $F_{2}$ [26], color-suppressed $B$-decays involving light isodoublets, such as $\bar{B}^{0} \rightarrow D^{0} \bar{K}^{0}$ and $\bar{B}^{0} \rightarrow D_{s} K^{-}$[27], and annihilation contributions in two-body $B$-decays [28, 29, 30]. Endpoint singularities also appear in non-exclusive processes such as semi-inclusive deep inelastic scattering at low transverse momentum [31, 32. When the zero-bin procedure is applied to these cases, individual pieces of the amplitudes exhibit dependence on a rapidity parameter.

In the work of Collins and Soper [33, 34], factorization formulas involving a rapidity parameter were derived for fragmentation in $e^{+} e^{-} \rightarrow A+B+X$ where $A$ and $B$ are hadrons. The separation of degrees of freedom in $\mathrm{SCET}_{\text {II }}$ gives finite amplitudes that appear to indicate that rapidity dependent factorization also occurs in other two-hadron processes, including purely exclusive ones. Our finite amplitudes are shown to be a direct consequence of defining the degrees of freedom in $\mathrm{SCET}_{\text {II }}$ carefully. We will not give a complete derivation of an exclusive rapidity factorization formula here, because in our analysis we will make the simplifying assumption that the renormalization of rapidity space effects and invariant mass effects can be carried out independently.

It is useful to have a physical understanding of why resolving the double counting issue also resolves the singularity problems. A hint comes from the fact that neither the pinch 
singularities or endpoint singularities are present in the full QCD computations, as pointed out for SCET in 35] in the context of $B \rightarrow \pi \ell \bar{\nu}$. For case 1) above, the soft pinch singularities are removed by the kinetic energy of the quarks, $1 /\left[k^{0}-\mathbf{k}^{2} /(2 m)\right]$. In case 7$)$ above, the endpoint singularities are soft limits of the full theory diagrams and are removed by $\Lambda_{\mathrm{QCD}}$, or in non-relativistic systems [36], by the binding energy. An improper interpretation of the singularities can occur by not being careful about taking double limits. In 1) the singularity arises from first taking $k^{0} \gg \mathbf{k}^{2} /(2 m)$ and then $k^{0} \rightarrow 0$. The $k^{0} \gg \mathbf{k}^{2} /(2 m)$ limit gives soft quarks which can not properly describe the potential-quark region where $k^{0} \sim \mathbf{k}^{2} /(2 m)$. Likewise, in 7) the endpoint singularity comes from first taking $k^{-} \gg k_{\perp}, k^{+}$and then taking $k^{-} \rightarrow 0$. The collinear particles obtained from $k^{-} \gg k_{\perp}, k^{+}$do not properly describe the soft particle region where $k^{-} \sim k_{\perp}, k^{+}$. To avoid double counting we must ensure that the soft quarks do not double count the potential region in case 1), and that the collinear quarks do not double count the soft region in 7). In the effective theory implementation of the expansion of QCD these singular limits are properly described by other degrees of freedom. Once we avoid the double counting, the unphysical singularities never appear because the potential limit of the soft quarks and the soft limit of the collinear quarks are rendered harmless. It should be emphasized that the pinch and endpoint singularities we are discussing are unphysical artifacts of certain approximations, and are reflected by double counting problem that must be fixed in the Effective Field Theory (EFT). They are not the same as the classification of physical IR divergences from the Landau Equations (see eg. [37, 38]) which go by similar names. The true IR structure of the full theory is properly reproduced by contributions from the full set of EFT degrees of freedom.

It is important to note that the zero-bin subtractions avoids double counting independent of the choice of UV and IR regulators in effective theory computations. Our implementation of zero-bin subtractions is unique up to possible finite scheme dependent contributions, and provides an explicit connection to methods which introduce hard factorization cutoffs. It also provides a definition of the modes in these effective theories independent of dimensional regularization (and with some work could be used for example to take the cutoff formulation of SCET described below and implement it on the lattice). Since the proper formulation of an EFT should not depend on the choice of IR regulator used in perturbative computations, this is not surprising. ${ }^{1}$ Physical results in QCD are IR finite with divergences removed by quantities like $\Lambda_{\mathrm{QCD}}$, binding energies, or cancellations between real and virtual diagrams, and the same is true in the EFT.

The outline of the paper is as follows. In section $\mathbb{I}$ we give a brief introduction to NRQCD and SCET which serve as our main examples. The tiling of IR regions with modes

\footnotetext{
${ }^{1}$ The choice of IR or UV regulators can make it more difficult to perform the power counting, for example by leading to integrals that are not homogeneous and require power counting violating counterterms to give back a power counting for the renormalized graphs.
} 
is discussed in section III, and the zero-bin subtraction is formulated in section IV. Our discussion is in the context of NRQCD and SCET, but is general enough to be readily adapted to other physical situations. In section $\nabla$ we give examples in NRQCD to show that the zero-bin removes pinch singularities, implies the pullup mechanism, and avoids double counting problems. In section $\nabla \mathbb{T}$ we give examples in $\mathrm{SCET}_{\mathrm{I}}$ which demonstrate the regulator independence of the zero-bin method, and the removal of double counting in loop integrals and in inclusive phase space computations. Finally, in section $V I T$ we give examples in $\mathrm{SCET}_{\mathrm{II}}$ which is formulated with zero-bin subtractions and only soft and collinear modes. The zero-bin subtraction resolves the endpoint singularity issue in exclusive processes to leave finite amplitudes, and require the introduction of a rapidity parameter. Conclusions are given in section VIII.

\section{NRQCD AND SCET}

NRQCD is an effective theory for non-relativistic quark-antiquark $(Q \bar{Q})$ bound states, where the typical relative fermion velocity, $v$, is small, $v \ll 1$. The relevant scales in NRQCD are the quark mass, $m$, momentum $p \sim m v$, and energy $E \sim m v^{2}$, with $E \ll p \ll m$. The energy and momentum are not independent; they are coupled via the quark equation of motion, $2 m E=p^{2}$.

SCET describes the interaction of energetic particles; examples include the inclusive decay $B \rightarrow X_{s} \gamma$ at large $E_{\gamma}$ via the partonic decay $b \rightarrow s \gamma$, inclusive jet production, or exclusive semileptonic decays such as $B \rightarrow \pi \ell \bar{\nu}$. It is convenient to orient the coordinate system in the direction of the energetic jet or hadron, for example by introducing null vectors $n=(1,0,0,1)$ and $\bar{n}=(1,0,0,-1)$, and use light-cone coordinates with $p^{+} \equiv n \cdot p, p^{-} \equiv \bar{n} \cdot p$ for any fourvector $p$. Energetic particles moving near the $n$ direction have momenta $p^{-} \sim Q, p^{+} \sim Q \lambda^{2}$ and $\mathbf{p}_{\perp} \sim Q \lambda$, where $\lambda \ll 1$ and $Q \gg \Lambda_{\mathrm{QCD}}$. $Q$ is the large energy scale, and is of order $m_{b}$

for $B$-decays. Often the choice $\lambda \sim \sqrt{\Lambda_{\mathrm{QCD}} / Q}$ is made for inclusive processes in $\mathrm{SCET}_{\mathrm{I}}$, but parametrically larger choices for this small parameter are allowed. For exclusive processes in $\mathrm{SCET}_{\mathrm{II}}$ we use $\eta$ rather than $\lambda$ for the expansion parameter to avoid confusion, since here $\eta \sim \Lambda_{\mathrm{QCD}} / Q$. The $p^{+}, p^{-}$and $\mathbf{p}_{\perp}$ scales are coupled by the on-shell condition $p^{+} p^{-}=\mathbf{p}_{\perp}^{2}$, and despite the hierarchy $p^{-} \gg p_{\perp} \gg p^{+}$, the effective theory must simultaneously deal with the low energy scales associated with $p_{\perp}$ and $p^{+}$.

\section{A. Comparison with the NRQCD Method of Regions}

One can evaluate Feynman integrals in NRQCD using the method of regions [13] (also called the threshold expansion), which divides up an integral into hard, soft, potential and usoft contributions based on scaling for the loop momentum. The idea is that in dimensional 
regularization the sum of these contributions exactly reproduces the full theory diagram, so

$$
A^{\text {full }}\left(p_{i}\right)=\prod_{j} \int d^{d} k_{j} F\left(p_{i}, k_{j}\right)=\sum_{\text {regions } \ell} \prod_{j} \int d^{d} k_{j} F^{(\ell)}\left(p_{i}, k_{j}\right) .
$$

Dimensional regularization is required here because there are cancellations between UV and IR divergences from different regions, which occur if $\epsilon_{\mathrm{IR}}=\epsilon_{\mathrm{UV}}=\epsilon$. Eq. (田) does not define an effective field theory, but it is sometimes taken as a way of defining EFT contributions to amplitudes, by demanding that in dimensional regularization each mode in the effective Lagrangian should reproduce a term from a region on the RHS. Although Eq. (四) is quite powerful, a few points must be treated carefully: i) The division of regions is gauge dependent. $^{2}$ ii) The requirement that scaleless integrals be set to zero, $1 / \epsilon_{\mathrm{UV}}-1 / \epsilon_{\mathrm{IR}}=0$, does not allow all UV divergences to be treated by counterterms, nor a verification that every IR divergence has a correspondence with the full theory. iii) In Eq. (丰) one must sum over all possible momentum routings in loops to determine the relevant regions (or consider the scaling of all combinations of loop momenta and external momenta). This is because it is individual propagators in the EFT that belong to a region rather than the loop momenta.

In the remainder of this section we will explore how the terms $\left(1 / \epsilon_{\mathrm{UV}}-1 / \epsilon_{\mathrm{IR}}\right)$ allow a residual freedom in associating amplitudes with degrees of freedom beyond that in Eq. (价. The treatment of these terms effects the correspondence of the EFT modes with physical regions. Lets consider a one-loop graph in NRQCD with contributions from different regions. The soft contribution depends on external soft scales such as the momentum transfer $\mathbf{r}$, and has the (schematic) form

$$
I_{\mathrm{soft}}=\frac{A}{\epsilon_{\mathrm{UV}}}+\frac{B}{\epsilon_{\mathrm{IR}}}+f(\mathbf{r}, \mu)
$$

As the momentum in the soft graphs vanishes, the graph matches on to an usoft diagram, with the (schematic) structure

$$
I_{\mathrm{usoft}}=-\frac{B}{\epsilon_{\mathrm{UV}}}+\frac{C}{\epsilon_{\mathrm{IR}}}+g(E, \mu),
$$

where the coefficient $B$ is the same as in Eq. (5). The usoft graph depends on external usoft scales such as the energy $E$. The IR divergences in the usoft sector are true IR divergences. They arise if one is computing an IR divergent quantity such as an on-shell Green's function, but cancel in measurable quantities such as physical scattering cross-sections and bound state energies.

The IR divergence in the soft graphs and the ultraviolet divergence in the usoft graph are at the intermediate scale $m v$ and cancel each other; they are not true divergences of the

\footnotetext{
${ }^{2}$ A well known example in NRQCD is applying the method of regions to potential and soft contributions. Another example is the division between $(E, \mathbf{p}) \sim(m, m)$ and a new region $(E, \mathbf{p}) \sim(m, m v)$ that shows up at fourth order in the $v$ expansion in Coulomb gauge.
} 

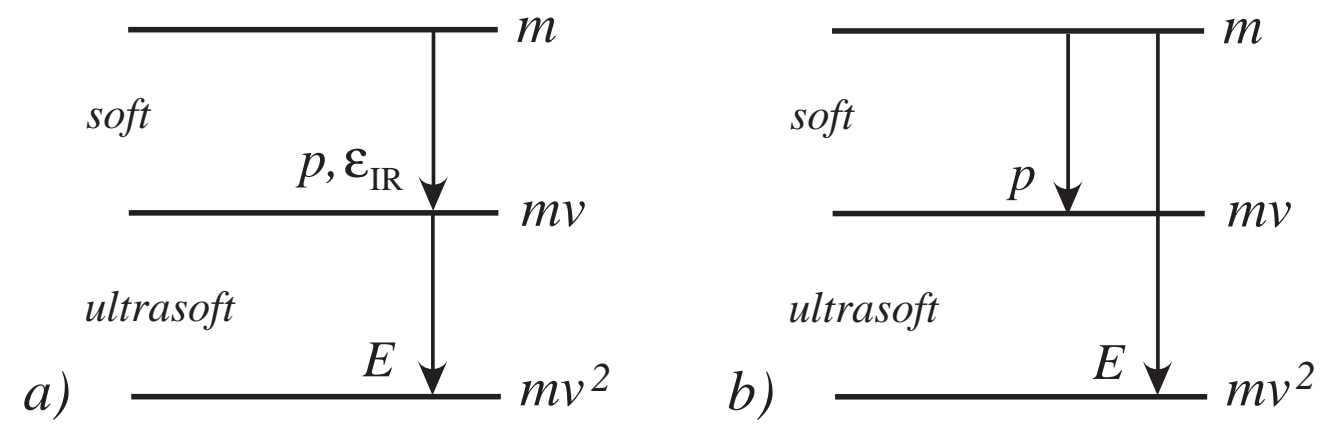

FIG. 1: Comparison of two setups for the soft and usoft contributions to an NRQCD Feynman graph. In a) the $1 / \epsilon$ divergences at the intermediate scale $m v$ cancel between the soft and usoft contributions. In b) there are no IR divergences at this intermediate scale.

theory. Since in the method of regions a rule is applied that scaleless integrals are set to zero, one is free to consider the $B / \epsilon$ terms canceling in the sum of Eqs. (5.,6) to give

$$
I_{\mathrm{soft}}+I_{\mathrm{usoft}}=\frac{A}{\epsilon_{\mathrm{UV}}}+\frac{C}{\epsilon_{\mathrm{IR}}}+f(\mathbf{r}, \mu)+g(E, \mu) .
$$

This interpretation leads to the picture shown in Fig. 17a, where the IR soft effects and UV ultrasoft effects meet at $m v$. Thus this particular mapping of the method of regions with effective theory amplitudes does not lead to simultaneously having degrees of freedom for soft and usoft gluons at a scale $\mu$, but instead in the renormalization group the soft contribution covers the momentum region between $m$ and $m v$ and the usoft contribution covers the region between $m v$ and $m v^{2}$.

In an effective field theory, the low energy effective Lagrangian is usually required to reproduce all the IR effects of the original theory from the start. In NRQCD, this plus a manifest power counting in $v$ requires that the effective theory include soft and usoft degrees of freedom at the same time to reproduce the dependence of the full theory on the momentum transfer $\mathbf{r} \sim m v$ and the energy $E \sim m v^{2}$, which are considered IR scales at $\mu=m$ where the effective Lagrangian is constructed, as found in Ref. [8]. This happens because the power counting links the scales $m v$ and $m v^{2}$. A similar result holds for SCETthe SCET Lagrangian at the hard scale $Q$ must simultaneously include collinear and usoft degrees of freedom to correctly reproduce the IR behavior of QCD with a power counting in $\lambda$ [1, 2]. In the effective theory, counterterms must be added for all $1 / \epsilon_{\mathrm{UV}}$ divergences, including those from scaleless integrals as is familiar from the study of HQET [39, 40, 41]. The counterterm structure can not depend on the choice of IR regulator.

The above line of reasoning gives the picture Fig. 1]b, rather than Fig. 1 $1 \mathrm{a}$. In NRQCD this picture has been implemented in the past by a procedure referred to as a pullup mechanism [14, 15]. One modifies Fig. 11a by pulling up the usoft modes to the scale $m$, and then subtracting the usoft contribution from the soft contribution in the region between $m$ and $m v$. The picture now looks like Fig. 1 $1 \mathrm{~b}$. This modification leaves the usoft contribution 
Eq. (6) with the same form, but it must now be included in the theory between the scales $m$ and $m v^{2}$. The usoft integral in the region between $m$ and $m v$ has the form

$$
I_{0}=-\frac{B}{\epsilon_{\mathrm{UV}}}+\frac{B}{\epsilon_{\mathrm{IR}}}
$$

so it corresponds to a $\left(1 / \epsilon_{\mathrm{UV}}-1 / \epsilon_{\mathrm{IR}}\right)$ that we are free to move between amplitudes from the point of view of Eq. (田). When Eq. (8) is subtracted from Eq. (5) it gives the modified soft contribution

$$
I_{\mathrm{soft}}=\frac{A}{\epsilon_{\mathrm{UV}}}+\frac{B}{\epsilon_{\mathrm{UV}}}+f(\mathbf{r}, \mu)
$$

The IR divergent parts of the soft graphs have been removed. There is no need for any cancellation of divergences between the soft and usoft degrees of freedom, and they can be renormalized separately. This is important, because it is known that the soft and usoft degrees of freedom have independent coupling constants, and the cancellation of divergences between the two can be problematic in renormalization group improved perturbation theory. One of the consequences of the pullup mechanism is that the anomalous dimensions in the soft sector are given by Eq. (9), and are proportional to $A+B$. As a short cut one can compute the anomalous dimension from the original form in Eqs. (5,6) by treating the IR divergence as though it were a UV divergence in Eq. (5), and taking the UV divergence in Eq. (6) to be at the hard scale. This is the procedure that has been followed in previous NRQCD and NRQED computations [8, 10, 14, 15, 42, 43, 44, 45, and is known to be necessary to correctly reproduce the high-order logarithmic terms in Lamb shifts and hyperfine splittings which are determined from independent fixed order QED computations. We will see that the zero-bin subtraction automatically gives the final result implied by the pullup mechanism, so that the soft integrals with the zero-bin subtraction have the form in Eq. (9), rather than that in Eq. (5). Thus the zero-bin formulation no longer requires implementing a pullup by hand.

An alternative setup has been explored in Ref. 446, 47, 48, 49, which keeps Fig. 17a but postpones obtaining power counting in $v$. In this setup one starts by matching onto a purely soft theory with a power counting in $1 / m$ and constrains states to $E=0$ so the IR divergences are properly reproduced. The power counting in $v$ is not yet manifest in this theory. The soft theory is then matched at a soft cutoff scale $\Omega_{s}$ onto an usoft theory, pNRQCD, where $E \neq 0$ and the velocity power counting is restored. The key is to maintain $\Omega_{s}$ as a variable in the usoft theory so that when required it can be run down from the scale $m$ including the required correlation with the usoft scale. While this setup seems to differ from the pullup, in several cases it has been shown that both methods give equivalent final answers for observables with log summation.

Note that so far an analogous setup that would avoid simultaneously introducing usoft and collinear modes does not exist for $\mathrm{SCET}_{\mathrm{I}}$. It is known that one can avoid simultaneously 
introducing hard-collinear $p^{2} \sim Q \Lambda_{\mathrm{QCD}}$ and collinear $p^{2} \sim \Lambda_{\mathrm{QCD}}^{2}$ modes, by matching $\mathrm{SCET}_{\mathrm{I}}$ onto $\mathrm{SCET}_{\mathrm{II}}$ as discussed in Ref. [35]. This postpones obtaining the final power counting until one matches onto $\mathrm{SCET}_{\mathrm{II}}$, and the (hard-collinear)-(collinear) setup is similar to the soft-usoft setup in pNRQCD. Just as in pNRQCD one must in general maintain the matching scale as a free parameter with this method of matching $\mathrm{SCET}_{\mathrm{I}}$ onto $\mathrm{SCET}_{\mathrm{II}}$. Alternatively, one can match QCD directly onto $\mathrm{SCET}_{\mathrm{II}}$ [4, 50].

\section{B. Summing Logarithms}

The dynamical relations $p^{2}=2 m E$ and $p^{+} p^{-}=\mathbf{p}_{\perp}^{2}$ have implications for the summation of logarithms using renormalization group evolution. In NRQCD and NRQED, one must simultaneously run from $m \rightarrow p$ and $m \rightarrow E$ in the soft and usoft sectors of the theory [8]. This is implemented in practice by using the velocity renormalization group [8]. Graphs in the theory are evaluated using two different $\mu$ parameters, $\mu_{S}$ for soft and potential loops, and $\mu_{U}$ for usoft loops. One then sets $\mu_{S}=m \nu, \mu_{U}=m \nu^{2}$, and runs from $\nu=1$ to $\nu=v$. This procedure is also referred to as one-stage or correlated running [51, and corresponds to Fig. 1 $1 \mathrm{~b}$. In NRQED, this correlated running is required to correctly compute the $\alpha^{8} \ln ^{3} \alpha$ Lamb shift and $\alpha^{7} \ln ^{2} \alpha$ hyperfine splittings for Hydrogen and positronium, as well as the $\alpha^{3} \ln ^{2} \alpha$ positronium widths 443, 51]. It has also been shown to be necessary to properly implement counterterms in subdivergences at three-loops [44, 52]. In some cases, correlated running is not essential, and one can follow an alternative procedure called twostage or uncorrelated running, in which $\mu_{S}$ is scaled from $m$ to $m v$, and $\mu_{U}$ from $m v$ to $m v^{2}$, corresponding to Fig. [a. ${ }^{3}$ The summation of logarithms for the $1 / \mathrm{m}^{2}$ QCD potentials can be done with or without the correlated running [42, 47, 53]; both methods give the same result.

So far no examples where correlated running is essential have been encountered in SCET. ${ }^{4}$ This does not mean that correlated running is not necessary in SCET. In NRQED, correlated running is first required for computations of recoil corrections at order $v^{3}\left(m_{e} / m_{p}\right.$ terms in the Lamb shift), because it is at this order that the potential and usoft divergences are tied together. Until this order, both correlated and uncorrelated running give the same result, and it is the Lamb shift computation which shows that, in general, one should use correlated running. Thus, it is very likely that only the running of subleading factorization formulas in SCET will demonstrate the manner in which correlated running is required.

\footnotetext{
${ }^{3}$ See Ref. [51] for the precise relation between these two methods. The anomalous dimensions have different definitions in the two approaches, so the single $\ln$ terms agree. There is a difference only for $\ln ^{2}$ and higher terms if the anomalous dimension does not factor into separate soft/potential and usoft pieces.

${ }^{4}$ See the discussion in Ref. [18] on the equivalence of correlated and uncorrelated running at leading order for $B \rightarrow X_{s} \gamma$ and $e^{+} e^{-} \rightarrow J / \psi X$.
} 


\section{INFRARED MODES IN NRQCD AND SCET}

In its region of validity, an effective field theory needs to systematically reproduce the IR structure of the full theory order by order in its power expansion. In both NRQCD and SCET, a strict interpretation of this requirement makes it necessary to include distinct fields for different moment regions of the same physical particle. These distinct fields have different power counting. Multiple gluon fields were first introduced in Ref. [54 for potential and usoft gluons in NRQCD, with energy and momentum scaling of order $\left(E \sim m v^{2}, p \sim m v\right)$ and $\left(E \sim m v^{2}, p \sim m v^{2}\right)$, respectively. We will not introduce fields for potential gluons since they are not propagating degrees of freedom. In NRQCD it is also necessary to introduce soft gluons with momentum scaling $(E \sim m v, p \sim m v)$ [13, 55]. We use the NRQCD Lagrangians defined as in 9, 54] with potential quarks and usoft gluons/quarks, and also soft quarks. We treat soft gluon vertices with a soft-HQET effective Lagrangian, rather than integrating out the soft quarks as in Ref. [54]. At two-loops and beyond it is important to keep track of the $i 0^{+}$in the soft quark propagators $1 /\left(v \cdot k+i 0^{+}\right)$and leaving them in an action facilitates this. In SCET one requires both collinear and usoft gluons in a theory often called SCET $\mathrm{I}$ or collinear and soft gluons in a theory called SCET II . We use the Lagrangians from Ref. [4] for these theories.

One might expect that introducing multiple fields for the same particle would lead to double-counting problems. In constructing effective theories, one needs to know not only the power counting for the degrees of freedom, but also understand the range of scales for which these modes are included in the effective Lagrangian. If degrees of freedom overlap in some region of momentum space, an understanding of how their definitions avoid double counting is necessary. In the effective theories we study, loop integrals are dominated by external momenta by construction, so the power counting guarantees that fields give contributions that can overlap only in UV or IR limits. ${ }^{5}$ As a simple toy example consider Fig. 2. We imagine that there are two relevant momentum coordinates $p_{1}$ and $p_{2}$, and that physically there are four interesting sets of momenta labeled $q_{a}, q_{b}, q_{c}$, and $q_{d}$ which could be set kinematically or by bound state dynamics. The hard cutoffs $\Lambda_{1}$ and $\Lambda_{2}$ distinguish the momentum regions dominated by these $q$ 's.

Consider first the simplified case where we ignore the $p_{2}$ axis, and only have $q_{a}$ and $q_{b}$. This situation applies to many physical problems, including that of integrating out massive particles like the $W$-boson or $b$-quark. Here $q_{b}$ denotes hard fluctuations that are integrated out into Wilson coefficients $C$, while $q_{a}$ denotes low energy IR modes. To simplify the renormalization group evolution and leave symmetries unbroken, it is convenient to trade $\Lambda_{1}$ for a scaleless regulator such as dimensional regularization. The low energy theory has

\footnotetext{
${ }^{5}$ The statement actually holds for any renormalization procedure that respects the power counting, or at worst requires power counting violating counterterms.
} 


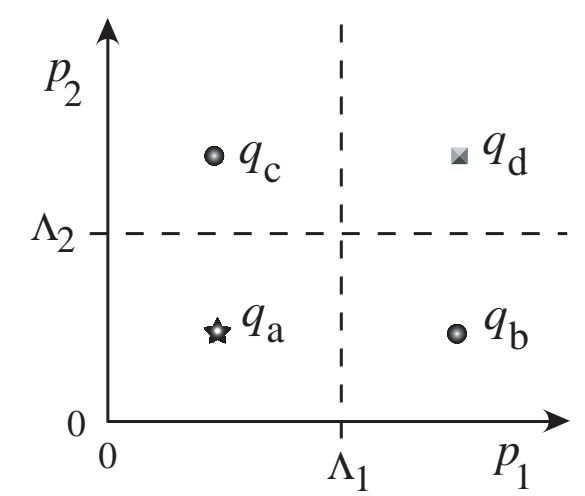

FIG. 2: Toy model to illustrate the scales captured by an effective theory with multiple low energy modes (here $q_{a}, q_{b}$, and $q_{c}$ ).

$C=C\left(q_{b}, \mu\right)$ where $\mu$ is the dimensional regularization parameter. The effective theory for $q_{a}$ with a scaleless regulator takes $\Lambda_{1} \rightarrow \infty$, and thus overlaps with the hard region of momentum space, but only through ultraviolet effects. In the effective theory this double counting is removed by UV counterterms $\propto 1 / \epsilon$, as well as through finite terms in the Wilson coefficients.

Now consider both the $p_{1}$ and $p_{2}$ axis. Here $q_{d}$ denotes hard fluctuations, and $q_{a}, q_{b}$, and $q_{c}$ are all low energy modes because they border a region where one or both of $p_{1,2}$ can be zero. Using dimensional regularization for each of these modes effectively takes $\Lambda_{1,2} \rightarrow \infty$ for $q_{a}$; $\Lambda_{1} \rightarrow 0, \Lambda_{2} \rightarrow \infty$ for $q_{b}$; and $\Lambda_{2} \rightarrow 0, \Lambda_{1} \rightarrow \infty$ for $q_{c}$. Again there is double counting in the UV which is taken care of by counterterms. However, there is now also a double counting in the IR. For example, taking $\Lambda_{1} \rightarrow 0$ for $q_{b}$ runs into the region for $q_{a}$. The $\Lambda_{1} \rightarrow 0$ limit is necessary for any scaleless regulator. In fact this limit is actually important physically, because we would like to define $q_{b}$ without reference to $q_{a}$ in order for it to be possible that contributions from $q_{a}$ and $q_{b}$ can factorize into independent well defined objects. This double counting in the IR is removed by the pullup mechanism, which as we will demonstrate, is a consequence of a proper treatment of the zero-momentum bin, namely $p_{2}=0$ for $q_{c}$ and $p_{1}=0$ for $q_{b}$. In pure dimensional regularization these zero-bin contributions amount to a correct interpretation of terms $\propto 1 / \epsilon_{\mathrm{UV}}-1 / \epsilon_{\mathrm{IR}}$. For example, $1 / \epsilon_{\mathrm{UV}}$ terms must be canceled by counterterms while $1 / \epsilon_{\mathrm{IR}}$ terms match up with IR divergences from the full theory.

We now turn to realistic effective theories and their zero-bin's. For NRQCD the modes are shown in Fig. 3a. The hard scale is $E \sim m$ or $\mathbf{p} \sim m$. We have propagating soft and usoft gluons/light quarks with power counting $E \sim \mathbf{p} \sim m v$ and $E \sim \mathbf{p} \sim m v^{2}$ respectively, and potential heavy quarks with $E \sim m v^{2}$ and $\mathbf{p} \sim m v$, and soft heavy quarks with $E \sim \mathbf{p} \sim m v$. Hard cutoffs $\Lambda, \Lambda_{1}$, and $\Lambda_{2}$ have been introduced to facilitate the discussion. Here $\Lambda \sim m$ is an ultraviolet scale below which one uses the effective theory, and $\Lambda_{1,2}$ divide up the low energy modes. These cutoffs will be removed exactly as in the toy example above. First consider the soft and usoft gluons. In a theory with both present, the energy/momentum 


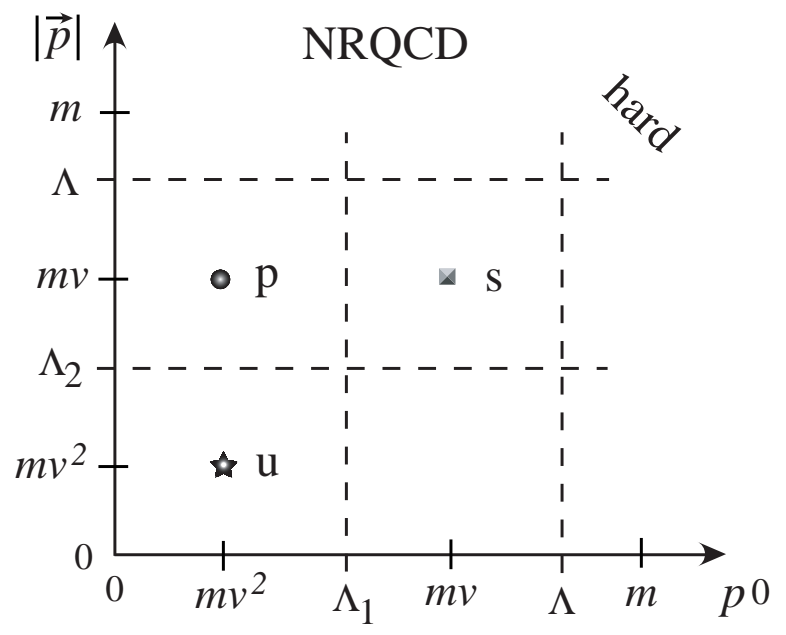

FIG. 3: a) Scales and momentum modes for nonrelativistic field theories like NRQCD. Here s, p, and $u$ denote soft, potential, and usoft respectively.

regions we want them to cover are

$$
\begin{array}{lrl}
\text { soft gluons: } & \Lambda \gg m v & \gtrsim \Omega^{s}, \\
\text { usoft gluons: } & \Lambda \gg m v^{2} \gtrsim \Omega^{u},
\end{array}
$$

where $\Omega^{s}$ and $\Omega^{u}$ are soft and usoft scales denoted by the box and star in Fig. 3 . These scales are usually set by external variables such as the momentum transfer or energy of the quarks, or by the nonperturbative scale $\Lambda_{\mathrm{QCD}}$. In order to reproduce all possible IR effects associated with $E \sim m v^{2}$, the EFT necessarily must have usoft modes just below the UV scale $\Lambda$. The division in Eq. (10) also implies that the UV divergences associated with soft and usoft modes contribute to the same anomalous dimension. This setup corresponds to the result obtained from the same limits for the cutoffs $\Lambda_{1,2}$ as discussed in the toy model above. Now double counting must be avoided in the region between $\Lambda$ and $\Omega^{s}$, between the IR of the soft gluons and the ultraviolet of the usoft gluons (i.e. when virtual momenta for the soft gluons becomes comparable to virtual momenta for the usoft gluons in loops).

A line of reasoning that gets close to seeing how this is achieved is to start with soft gluons over the interval $\Lambda \gg m v \sim \Lambda_{1}$ and usoft gluons over $\Lambda_{1} \gg m v^{2} \sim \Omega^{u}$, where we set $\Lambda_{2}=\Lambda_{1}$. Here $\Lambda_{1}$ is considered as an intermediate factorization scale [46, 47, 48]. Next one adds a pullup contribution to the usoft gluons and simultaneously subtracts the same contribution from the soft gluons as described in Refs. [14, 15]. This pulls the upper limit $\Lambda_{1}$ for the usoft gluons all the way up to the scale $\Lambda$, while at the same time avoiding double counting between $m$ and $m v$. The pre-pullup setup is shown in Fig. 17a and post-pullup in Fig. [1b. Taking into account the zero-bin gives the post-pullup setup. With the pullup, the contributions from soft and usoft gluons are now as in Eq. (10). If the cutoffs had been swapped for a scaleless regulator, this would amount to a proper interpretation of $1 / \epsilon$ poles as discussed in Ref. [15]. 
In this paper we show that Eq. (10) is obtained automatically by carefully considering the zero-bin in the NRQCD effective Lagrangian. The usoft gluons are defined all the way up to $\Lambda$, and the subtraction for the soft gluons is associated with properly removing their zero momentum bin. Thus the effective Lagrangian gives the full procedure for the evaluation of Feynman graphs and is decoupled from the additional choice of which regulators to use. With the zero-bin taken into account there is no longer a need to implement a separate pullup.

A second type of division between potential and soft IR modes in NRQCD is also shown in Fig. Ba. In this case the distinction is solely in the energy variable $p^{0}$. Potential gluons are not propagating degrees of freedom since they have $p^{0} \psi \mathbf{p}$, and so potential gluon fields should not be introduced (they would have problems with gauge invariance for example). The matching of soft gluons onto four-quark operators with potential coefficients can be thought of in a similar manner to integrating out a massive particle [56]. On the other hand a zero-bin subtraction is necessary to distinguish soft and potential quarks. Their momentum regions and propagators are

$$
\begin{array}{lll}
\text { soft quarks: } \quad\left(p^{0}: \Lambda>p^{0} \sim m v>\Omega^{s}\right) ; & \text { propagator: } \frac{i}{\left[p^{0}+i 0^{+}\right]}, \\
\text {potential quarks: } \quad\left(p^{0}: \Lambda>p^{0} \sim m v^{2}>\Omega^{u}\right) ; \quad \text { propagator: } \frac{i}{\left[p^{0}-\frac{\mathbf{p}^{2}}{2 m}+i 0^{+}\right]} .
\end{array}
$$

Without the zero-bin, double counting occurs when $p^{0} \rightarrow 0$ in the soft propagator, and this reveals itself through the presence of pinch singularities in soft loop diagrams with quarks and antiquarks, which have the form

$$
\int \mathrm{d} p^{0} \frac{1}{\left[p^{0}+i 0^{+}\right]\left[-p^{0}+i 0^{+}\right] \cdots} .
$$

Here we will show that the zero-bin subtraction removes all pinch singularities in the soft regime. At the same time it avoids double counting of soft and potential contributions. It is useful to recall that the same is not true in the method of regions [13], where the method used to avoid the pinch singularities is to consider the expansion only after doing the $p^{0}$ integral by contours.

Note that in NRQCD there is no issue of a possible potential-usoft overlap for quarks since propagating usoft quarks are light quarks and therefore of a different flavor from the potential quarks.

So far the discussion was for NRQCD, but similar logic holds for $\mathrm{SCET}_{\mathrm{I}}$. In this case the hard scale is set by $Q$ and the expansion parameter is $\lambda$. Below the scale $Q$ we have usoft and collinear gluons with power counting $\left(p^{+}, p^{-}, p^{\perp}\right) \sim Q\left(\lambda^{2}, \lambda^{2}, \lambda^{2}\right)$ and $Q\left(\lambda^{2}, 1, \lambda\right)$ respectively. These gluons cover the regions

$$
\begin{array}{ll}
\text { usoft gluons: } \quad\left(p^{-}: \Lambda \gg Q \lambda^{2} \gtrsim \Omega^{u}\right) ; \quad\left(p^{\perp}: \Lambda \gg Q \lambda^{2} \gtrsim \Omega^{u}\right), \\
\text { collinear gluons: } \quad\left(p^{-}: \Lambda>Q \lambda^{0} \gtrsim \Omega_{-}^{c}\right) ; \quad\left(p^{\perp}: \Lambda \gg Q \lambda \gtrsim \Omega_{\perp}^{c}\right),
\end{array}
$$



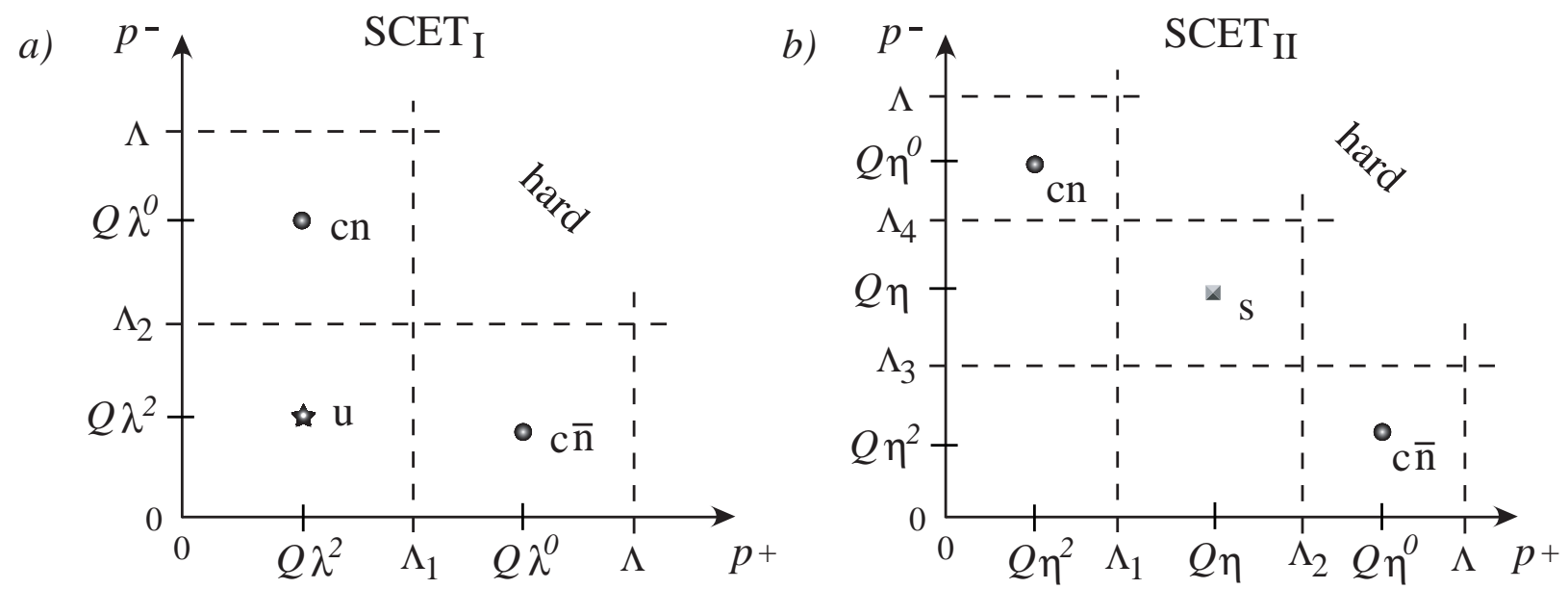

FIG. 4: a) Scales and momentum modes for $\mathrm{SCET}_{\mathrm{I}}$. Here $c n, c \bar{n}$, and $u$ denote collinear- $n$, collinear- $\bar{n}$, and usoft modes respectively and $\lambda \sim \sqrt{\Lambda_{\mathrm{QCD}} / Q}$. b) Scales and momentum modes for $\operatorname{SCET}_{\mathrm{II}}$. Here $c n, c \bar{n}$, and $s$ denote collinear- $n$, collinear- $\bar{n}$, and soft modes respectively and $\eta \sim \Lambda_{\mathrm{QCD}} / Q$.

and a common region for $p^{+}: \Lambda \gg Q \lambda^{2} \gtrsim \Omega^{u}$. These regions are denoted by a circle and star in Fig. Ga, where we have also included a second collinear region $c \bar{n}$ for later convenience. In Eq. (13) we have used a common UV scale $\Lambda$ and a common usoft IR scale $\Omega^{u}$ in the and $\perp$ components which have power counting $\sim \lambda^{2}$. In $\mathrm{SCET}_{\mathrm{I}}$ we see that we must avoid double counting in the region $\Lambda$ to $\Omega_{-}^{c}$ for the $p^{-}$momenta, and the region $\Lambda$ to $\Omega_{\perp}^{c}$ for the $p^{\perp}$ momenta. ${ }^{6}$ As in NRQCD, this is achieved by a proper treatment of the zero momentum bin for collinear fields (which implements a pullup in SCET).

Finally, we can consider the theory $\mathrm{SCET}_{\mathrm{II}}$ which has soft and collinear gluons with power counting $\left(p^{+}, p^{-}, p^{\perp}\right) \sim Q(\eta, \eta, \eta)$ and $Q\left(\eta^{2}, 1, \eta\right)$ respectively, for $\eta \sim \Lambda_{\mathrm{QCD}} / Q$. Here the regions are

$$
\begin{array}{lll}
\text { soft gluons: } & \left(p^{-}: \Lambda \gg Q \eta \gtrsim \Omega^{s}\right) ; & \left(p^{+}: \Lambda \gg Q \eta \gtrsim \Omega^{s}\right), \\
\text { collinear gluons: } & \left(p^{-}: \Lambda>Q \eta^{0} \gtrsim \Omega_{-}^{c}\right) ; & \left(p^{+}: \Lambda \gg Q \eta^{2} \gtrsim \Omega_{+}^{c}\right),
\end{array}
$$

and for convenience a common region for $p^{\perp}: \Lambda \gg Q \eta \gtrsim \Omega^{s}$. Both soft and collinear modes describe non-perturbative fluctuations close to the mass shell, $p^{2} \sim Q^{2} \eta^{2} \sim \Lambda_{\mathrm{QCD}}^{2}$. Interactions between these modes are offshell [4] by an amount, $p_{h c}^{2} \sim Q^{2} \eta$, and dependence on this momentum is integrated out, appearing in the coefficient functions for mixed softcollinear operators. Here double counting in SCET II occurs when a collinear momentum overlaps the soft region, and when a soft momentum overlaps the collinear region. This case differs from our discussion of NRQCD and SCET $_{I}$ because here the overlapping modes have

\footnotetext{
${ }^{6}$ For typographical convenience we use $\Omega_{-}$although a superscript, $\Omega^{-}$, would be more appropriate.
} 
the same $p^{2}$, but differ in their rapidity $y$, or more conveniently their value of

$$
\zeta_{p}=e^{2 y}=\frac{p^{-}}{p^{+}}
$$

The $n$-collinear modes have $\zeta_{p} \gg 1$, the soft modes have $\zeta_{p} \sim 1$, and the $\bar{n}$-collinear modes have $\zeta_{p} \ll 1$. Consider a process for which $\bar{n}$-collinear modes are irrelevant. Double counting is avoided by a proper treatment of the zero-bins: the " $p^{-}=0$ bin" for $n$-collinear modes and the " $p^{+}=0$ bin" for soft modes. However, here double counting of a physical IR region in QCD requires a correlated change in the + and - momenta: $p^{-}$gets small while $p^{+}$ gets big for collinear, and $p^{-}$gets big while $p^{+}$gets small for soft. The implementation of zero-bin's in $\mathrm{SCET}_{\mathrm{II}}$ is discussed further in section VII.

The treatment of the zero-bin ensure that the double counting is removed in the infrared, and that the overlap in the ultraviolet is properly handled by renormalization in the effective theory irrespective of the choice of regulator. If dimensional regularization is used to regulate both the IR and UV, then the added contributions are scaleless loop integrals that appear to be zero. In logarithmically divergent integrals, this occurs because the integral is the difference of UV and IR divergences, $0=1 / \epsilon_{\mathrm{UV}}-1 / \epsilon_{\mathrm{IR}}$, since there is only one $\epsilon$. If these added contributions are ignored, then one must be careful to properly interpret the divergences as UV or IR. This conversion of IR to UV divergences has been used implicitly in much of the NRQCD and SCET literature. However, if one wants to fully understand the physical significance of certain divergences or use another regulator in the UV or IR then explicitly including the subtractions discussed here is necessary.

\section{ZERO-BIN SUBTRACTIONS}

We start by reviewing how the relevant momentum scales are separated in the effective theory using labeled fields. In NRQCD, one first removes the large mass $m$ of the quark (and antiquark) from the problem just as in heavy quark effective theory (HQET) [57. The total momentum of the quark, $P^{\mu}$, is written as the sum $P^{\mu}=m \mathrm{v}^{\mu}+q^{\mu}$, where $\mathrm{v}^{\mu} \equiv(1,0,0,0)$. This subtracts $m$ from all the energies. The residual momentum $q^{\mu}$ is much smaller than $m$, and contains the non-relativistic energy $E \sim m v^{2}$ and momentum $p \sim m v$ of the particle, where $v$ is a scaling parameter of order the typical relative velocity between the heavy quarks. This mixes different powers of $v$. As shown in Ref. [8], it is useful to make a further division of $q^{\mu}, q^{\mu}=p^{\mu}+k^{\mu}$, where $p^{\mu}$ is of order $m v$, and $k^{\mu}$ is of order $m v^{2}$. This second separation allows the power counting in $v$ to be manifest in the effective theory. The break-up of $q^{\mu}$ is shown schematically in Fig. 5a. One breaks momentum space into a discrete variable $\mathbf{p}$ of order $m v$, and a continuous variable $k^{\mu}$ of order $m v^{2}$. Often $k$ is referred to as the residual momentum. The discrete label $\mathbf{p}$ does not have a time-component for quarks, since the energy is of order $m v^{2}$. The entire $q^{\mu}$ momentum space is covered by integrating over $k^{\mu}$ and summing over the labels p. Quarks are described by fields $\psi_{\mathbf{p}}(x)$, with an explicit label 

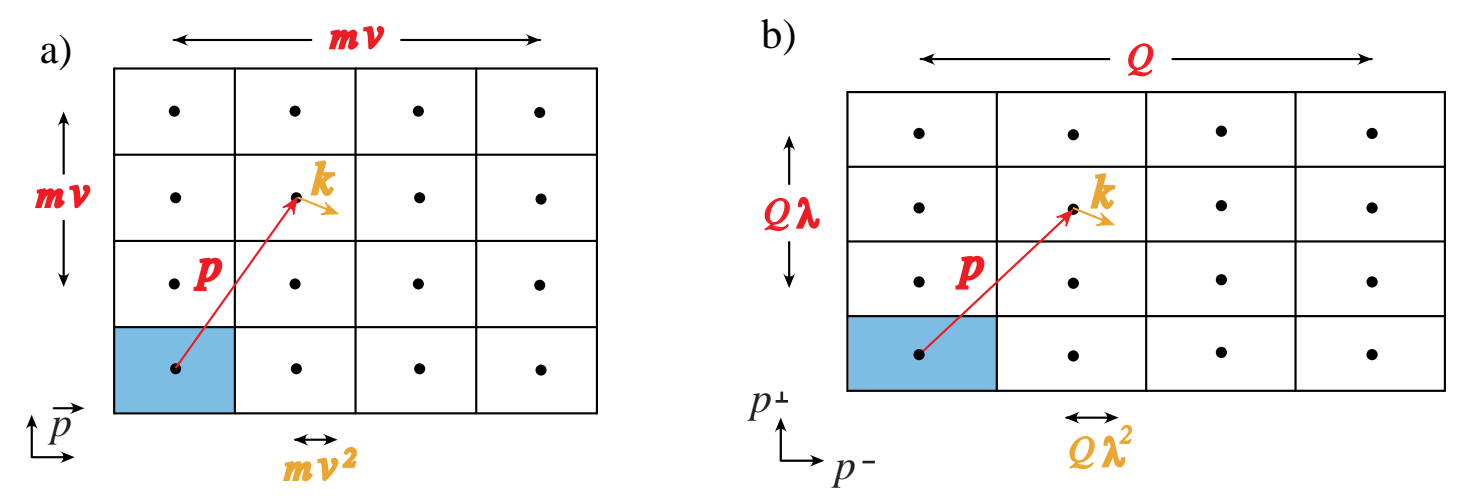

FIG. 5: Label and Residual momenta for a) NRQCD quarks and b) SCET I. In both cases $p$ denotes a large momentum, and labels a particular box, whereas $k$ is a small momentum, and gives the final momentum location relative to the reference momentum point in the box labeled by $p$.

$\mathbf{p}$, and the momentum $k^{\mu}$ is the Fourier transform of $x$. Similarly, soft gluons with energy and momentum of order $m v$ are described by gauge fields $A_{p}^{\mu}(x)$ with a four-vector label $p^{\mu}$. Ultrasoft gluons with energy and momentum of order $m v^{2}$ are described by gauge fields $A^{\mu}(x)$. Those unfamiliar with how the field theory with label and residual momenta works are referred to Ref. [54 or the example in the next section.

An analogous procedure was applied to SCET in Ref. [2, [3]. In SCET, one breaks up the collinear momentum into a label and residual momentum, $P^{\mu}=p^{\mu}+k^{\mu}$. The label $p$ contains the $Q$ and $Q \lambda$ pieces of the momentum, and the Fourier transform of the coordinate $x$ is the $Q \lambda^{2}$ part of the momentum $k$. Unlike NRQCD, the collinear interactions can still change the large label momentum $\sim Q$. In a theory referred to as $\mathrm{SCET}_{\mathrm{I}}$, one has collinear quark and gluon fields $\xi_{n, p}(x)$ and $A_{n, p}^{\mu}(x)$, where the label $p$ has minus and $\perp$ components, and usoft quark fields $A^{\mu}(x)$ which have energy and momentum of order $Q \lambda^{2}$. The $\mathrm{SCET}_{\mathrm{I}}$ decomposition is shown schematically in Fig. 㗘. In a theory called $\mathrm{SCET}_{\mathrm{II}}$ one has collinear quarks and gluons, $\xi_{n, p}, A_{n, p}^{\mu}$ with $p^{-}$label momenta, and soft quarks and gluons, $q_{s, k}, A_{s, k}^{\mu}$ with $k^{+}$label momenta. Here soft fields have plus-momenta much bigger than their collinear counterparts, and collinear fields have minus-momenta larger than their soft counterparts.

The quark and soft gluon fields in NRQCD and the collinear quark and gluon fields in SCET will be referred to as labeled fields. As is clear from Fig. 国, labeled fields must have a non-zero value for their label, i.e. they must be outside the zero-bin. Otherwise, they cover the same momentum region as the usoft fields. This is implemented at the level of the effective theory Lagrangian by requiring that all labeled fields in the Lagrangian have a nonzero value for the label. Terms in the effective theory Lagrangian have sums over the field labels, and all such sums are over non-zero values of the labels. One then has to carefully derive the rules for effective theory graphs including this constraint on the labels.

In loop integrals, one finds expressions which involve a sum over labels and an integral over the residual momentum, which can be converted to an integral over the entire momentum 
space [8],

$$
\sum_{p} \int \mathrm{d} k \longrightarrow \int \mathrm{d} p
$$

as is clear from Fig. 5. Here the label $p$ denotes a generic label, such as $\mathbf{p}$ for NRQCD quarks, $\left(\bar{n} \cdot p, \mathbf{p}_{\perp}\right)$ for collinear SCET quarks, etc. One residual momentum is removed for each sum over a label momentum. ${ }^{7}$ Any remaining residual momenta appear as integrals in their own right, providing a proper implementation of the multipole expansion. ${ }^{8}$ In

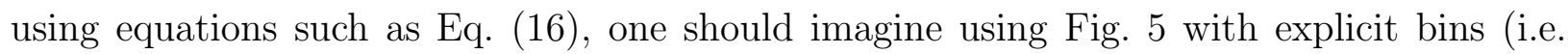
hard cutoffs) for the $k$ integrals. Once the final expressions are derived, one can evaluate the integrals in dimensional regularization, treating the bin sizes as infinite as discussed in section [II]. Eq. (16) is only true if we sum over all $p$, including $p=0$. The replacement in Eq. (16) is what we will call the result for the naive integral, generically denoted with a tilde, $\tilde{I}$.

We can finally formulate the zero-bin subtractions mentioned at the beginning of this article. The sum on $p$ in Eq. (16) is over $p \neq 0$ because the effective Lagrangian terms are a sum over $p \neq 0$. The restriction $p \neq 0$ in Eq. (16) modifies the right hand side. In a Feynman graph, let $F\left(\left\{p_{i}\right\},\left\{k_{i}\right\}\right)$ be the integrand, including all the momentum conserving $\delta$-functions and label preserving Kronecker- $\delta$ 's at the vertices, where $i$ runs over all the internal propagators. When integrating over a function $F$, the correct form of Eq. (16) is actually

$$
\sum_{\vee\left\{p_{i} \neq 0\right\}} \int \prod_{i} \mathrm{~d} k_{i} F\left(\left\{p_{i}\right\},\left\{k_{i}\right\}\right) \longrightarrow \int \prod_{i} \mathrm{~d} p_{i}\left[F\left(\left\{p_{i}\right\}\right)-\sum_{j \in U} F_{j}^{\mathrm{sub}}\left(\left\{p_{i}\right\}\right)\right] .
$$

On the l.h.s. the sum is over all label momenta avoiding the zero bins, $p_{i}=0$. On the r.h.s. we integrate $p_{i}$ over all of momentum space and the second term subtracts the contributions from regions $j \in U$ where one or more $p_{i}$ vanish. The set of such regions $U$ can be broken up into $U_{i}$ where $p_{i}=0, U_{i j}$ where $p_{i}=0$ and $p_{j}=0$, etc. The subtractions over $U$ are defined iteratively by first subtracting over each $U_{i}$, then adding back $U_{i j}$, subtracting $U_{i j k}$, etc. Note that since the $p_{i} \neq 0$ constraint comes from the fields in the Lagrangian, it is implemented at the level of propagators in a graph, i.e. for each internal line, not for each loop momentum. Once the momentum conserving delta functions are accounted for the subtractions are implemented at the level of the full integrand. ${ }^{9}$

\footnotetext{
${ }^{7}$ From the reparameterization invariance [58] in splitting $p+k$ it is equivalent to think of this as first fixing the lattice of $p$ 's and adding the integrals over $k$, or as using the freedom in the choice of $p$ 's to fix $k$ and then extending the sum over $p$ 's to an integral.

${ }^{8}$ See 59 for why this is relevant to power counting in NRQCD.

${ }^{9}$ If there are less sums over label momenta $p_{i}$ than integrals $d k_{i}$ then these extra integrals over $k$ 's will appear on the RHS with corresponding dependence in $F$. These extra $k$ 's are momenta that are truly small for the physical process, see Ref. [54]. For simplicity this complication was suppressed in writing Eq. (17), since it is not the most important aspect.
} 
Each of the $p_{i} \neq 0$ terms in Eq. (17) represent the full label on a field, which for example will be a four-vector for soft gluons in NRQCD, $q^{\mu}$, and the $q^{\mu}=q^{-} n^{\mu} / 2+q_{\perp}^{\mu}$ components for a collinear quark in $\mathrm{SCET}_{\mathrm{I}}$. The proper subtraction integrand $F^{\text {sub }}$ is obtained from $F$ by assigning a scaling to all the $p_{i}$ appropriate for the zero-bin region, and expanding in powers of the momentum which vanishes in the zero-bin (as we scale towards this region with the power counting parameter in the sense of a standard OPE). For example, if we sum over $q^{\mu} \neq 0$ for a soft momentum (i.e. order $m v$ ) in NRQCD, then we define $F^{\text {sub }}$ by an expansion of the integrand $F$ in powers of $q^{\mu}$ by assuming that $q^{\mu} \sim v^{2}$, i.e. by assuming, for the purposes of the expansion, that $q^{\mu}$ is usoft. This subtraction is done at the level of the integrand. The expansion is done to high enough order that the resulting integrand $F-F^{\text {sub }}$ vanishes in the zero-bin, i.e. vanishes as $q^{\mu} \rightarrow 0$.

Note that there is a freedom to define a scheme which leaves a finite integrand in the scaling limit since this just moves finite pieces around between the matching and matrix elements. We will use the scheme where all singularities are removed but not finite pieces. Also note that in a given subtraction some terms will be power divergences which in dimensional regularization are set to zero. This implies that if we had subtracted additional polynomial pieces they would not change the result of loop graphs in dimensional regularization since they integrate to zero.

To show that the RHS of Eq. (17) provides the proper implementation of the zero-bin independent of imposing hard momentum cutoffs we use a logic similar to section III in discussing Figs. 24. The $\sum_{p \neq 0}$ can be turned into a full integral if we add the $p=0$ bin, but we must subtract it again. The subtraction term has only an integral over residual momentum, but when we send the hard cutoffs on the sides of the zero-bin to $\infty$ the subtraction is also integrated over all momentum. Thus we end up subtracting terms derived from the scaling limit of the original integrand integrated over all of momentum space. The full integrand on the r.h.s. of Eq. (17) ensures that we do not double count the zerobin because the integrand vanishes when the loop momentum is sent towards the zero-bin momenta. This ensures there is no double counting in the IR. Any double counting in the UV is taken care of by Wilson coefficients and renormalization as usual.

It is worth emphasizing that the result in Eq. (17) applies equally well to the use of scaleless regulators like dimensional regularization, and to the case where hard Wilsonian cutoffs are applied to distinguish modes. For the Wilsonian case, consider the cutoffs as $\theta$-functions multiplying the integrand. In this situation the regulator ensures that the integrand is zero in the scaling limit so the subtraction terms all turn out to be zero, and the naive replacement in Eq. (16) with the cutoffs gives the correct answer.

The result in Eq. (17) applies to NRQCD or SCET or any other quantum field theory of this type. It is necessary to avoid double counting the zero-bin momenta which correspond to different degrees of freedom in the effective theory. It provides a means for tiling the infrared regions of a quantum field theory with different degrees of freedom while avoiding 
double counting. In the following sections we explore the difference between Eqs. (16) and (17), and its consequences, with the help of several examples.

Before leaving the general discussion it is worth emphasizing that Eq. (17) together with the definition of the NRQCD and SCET degrees of freedom given in Figs. 3 and 4 give complete coverage of all momentum regions where IR divergences can occur. We used multiple degrees of freedom to cover these IR regions because this facilitates setting up the proper EFT power counting expansion. In general one can look at combining regions together to describe a larger region with only a single degree of freedom. Doing so comes at the expense of making the power counting expansion difficult to formulate. In many cases it is actually unknown how to formulate the EFT expansion when regions are combined, thus necessitating multiple modes. We see that in general, the concepts of i) an EFT having a complete set of degrees of freedom to reproduce all IR divergences, and ii) the EFT having a valid power counting expansion, are tied together. We use this freedom to define NRQCD and SCET to cover the IR regions with our chosen degrees of freedom, so they reproduce the IR divergences. In this case proving that these EFT's are complete is equivalent to proving that their power counting expansions do not break down at any order. Demonstrating this is easier, since the power counting can only break down if we have missed a relevant operator at leading order in the expansion of some observable. All subleading operators are treated as insertions and do not upset the power counting. Thus, we see that constructing a complete EFT is equivalent to identifying the proper physical degrees of freedom in the leading order action, which is related to identifying the set of physical processes for which the EFT applies.

\section{ZERO-BIN SUBTRACTIONS IN NRQCD (NON-RELATIVISTIC PRO- CESSES)}

In this section we consider examples of the use of Eq. (17) for non-relativistic field theories. The results are quite general, applying whether the non-relativistic particles are quarks, nucleons, ions, or quasi-particles. The fields generating the potential are different in these cases, but the same momentum regions are important. To be definite we use a gauge theory, and so take our examples from non-relativistic QCD (NRQCD).

\section{A. NRQCD Soft Crossed-Box Graph}

It is helpful to consider a concrete example to study the consequences of Eq. (17) — we will start with the crossed-box graph in NRQCD. The full theory integral contains hard, soft, and usoft contributions, and we examine the soft crossed-box graph in the effective theory shown in Fig. 6. Here $\mathbf{p}_{1}$ and $\mathbf{p}_{2}$ are the external momenta, with $\mathbf{r}=\mathbf{p}_{2}-\mathbf{p}_{1}$ the

momentum transfer, and $2 m E=\mathbf{p}_{1}^{2}=\mathbf{p}_{2}^{2}$. The momentum of all the particles has been denoted as (label energy, residual energy; label momentum, residual momentum), and the 


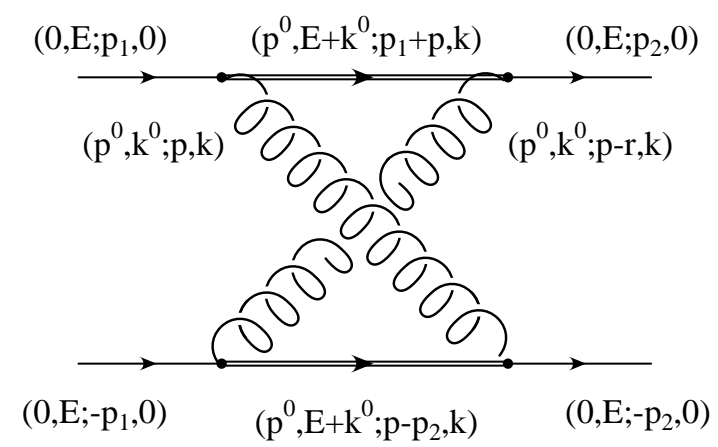

FIG. 6: Soft crossed-box graph in the effective theory. The zigzag lines are soft gluons, the double lines are soft quarks, and the single lines are potential quarks. For each line we show (label energy, residual energy; label momentum, residual momentum).

external particles have been chosen to have zero residual momentum.

In Feynman gauge the propagator for a soft gluon with momentum $\left(p^{0}, k^{0} ; \mathbf{p}, \mathbf{k}\right)$ is $1 /\left[\left(p^{0}\right)^{2}-\mathbf{p}^{2}+i 0^{+}\right]$, and for a soft quark with momentum $\left(p^{0}, k^{0} ; \mathbf{p}, \mathbf{k}\right)$ is $1 /\left[p^{0}+i \epsilon\right]$. We neglect overall factors such as color Casimirs and coupling constants, and focus on the integral for this graph:

$$
I_{S}^{\text {cross }}=\sum_{p^{0} \neq 0, p^{\mu} \neq 0, p^{\mu} \neq(0, \mathbf{r})} \int \frac{d^{D} k}{(2 \pi)^{D}} \frac{1}{p^{0}+i 0^{+}} \frac{1}{p^{0}+i 0^{+}} \frac{1}{\left(p^{0}\right)^{2}-\mathbf{p}^{2}+i 0^{+}} \frac{1}{\left(p^{0}\right)^{2}-(\mathbf{p}-\mathbf{r})^{2}+i 0^{+}}
$$

These soft propagators do not depend on the residual momentum components and so the propagator takes different values at each grid site in Fig. 3, but the same constant value for all the points in each box. Thus there is no change to the integrand when the label and residual momenta are combined into a continuous integration using Eq. (16), which gives

$$
\tilde{I}_{S}^{\text {cross }}=\int \frac{d^{D} p}{(2 \pi)^{D}} \frac{1}{p^{0}+i 0^{+}} \frac{1}{p^{0}+i 0^{+}} \frac{1}{\left(p^{0}\right)^{2}-\mathbf{p}^{2}+i 0^{+}} \frac{1}{\left(p^{0}\right)^{2}-(\mathbf{p}-\mathbf{r})^{2}+i 0^{+}} .
$$

However, Eq. ([19) still includes the zero-bin contribution, which must be subtracted out. A more careful analysis taking account of the zero-bin for each soft particle propagator, and instead using Eq. (17) implies that the value of the soft crossed-box graph is not Eq. (19) but rather

$$
I_{S}^{\text {cross }}=\tilde{I}_{S}^{\text {cross }}-I_{1}^{\text {cross }}-I_{2}^{\text {cross }}
$$

where the subtractions are: (a) $I_{1}$ from the region $\left(p^{0}=0, \mathbf{p}=0\right)$ and (b) $I_{2}$ from the region $\left(p^{0}=0, \mathbf{p}=\mathbf{r}\right)$ :

$$
\begin{aligned}
I_{1}^{\text {cross }} & =\int \frac{d^{D} p}{(2 \pi)^{D}} \frac{1}{p^{0}+i 0^{+}} \frac{1}{p^{0}+i 0^{+}} \frac{1}{\left(p^{0}\right)^{2}-\mathbf{p}^{2}+i 0^{+}} \frac{1}{-(\mathbf{r})^{2}+i \epsilon}, \\
I_{2}^{\text {cross }} & =\int \frac{d^{D} p}{(2 \pi)^{D}} \frac{1}{p^{0}+i 0^{+}} \frac{1}{p^{0}+i 0^{+}} \frac{1}{-\mathbf{r}^{2}+i 0^{+}} \frac{1}{\left(p^{0}\right)^{2}-(\mathbf{p}-\mathbf{r})^{2}+i 0^{+}} .
\end{aligned}
$$




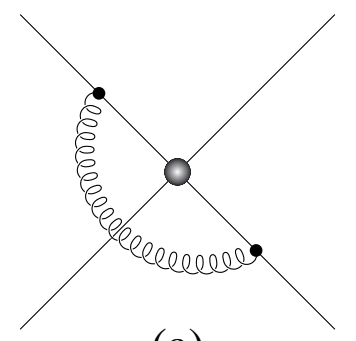

(a)

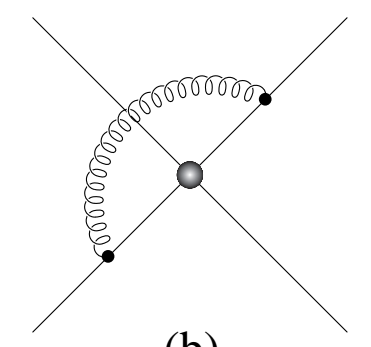

(b)

FIG. 7: Ultrasoft corrections to potential scattering related to the crossed box graph.

By the shift symmetry in $\mathbf{p}$ we have $I_{1}^{\text {cross }}=I_{2}^{\text {cross }}$.

The $I_{1}^{\text {cross }}$ subtraction comes from the region where the $\left(p^{0}, k^{0} ; \mathbf{p}, \mathbf{k}\right)$ gluon is usoft. Similarly, the $I_{2}^{\text {cross }}$ subtraction comes from the region where the other gluon becomes usoft. Subtractions from the region where the quark is potential $\left(p^{0}=0\right)$ vanish, as do the doublesubtractions where the regions for $I_{1,2}$ overlap with $p^{0}=0$. This is because for the crossed box all the $p^{0}$ poles are on the same side of the contour of integration.

The subtraction $I_{1}^{\text {cross }}$ avoids double counting the usoft graph shown in Fig. T(a) and similarly $I_{2}^{\text {cross }}$ avoids double counting the usoft graph Fig. $\mathbb{Z}(\mathrm{b})$. The usoft graphs depend on external usoft variables such as the energy. If these are set to zero, then the integral Fig. U(a) is $I_{1}^{\text {cross }}$, including the omitted color factors. The reason that $I_{1}^{\text {cross }}$ does not depend on external usoft variables, but Fig. U(a) could, is because $I_{1}^{\text {cross }}$ is obtained by considering a soft graph, and then taking its usoft limit. The effective field theory Feynman rules require that all usoft momentum be expanded out while considering soft diagrams. Thus $I_{1}^{\text {cross }}$ is the same as Fig. 7(a) with the external usoft variables expanded out.

Prior to the subtraction the soft crossed-box integral in dimensional regularization is

$$
\tilde{I}_{S}^{\text {cross }}=-\frac{i}{4 \pi^{2} \mathbf{r}^{2}}\left[\frac{1}{\epsilon_{\mathrm{IR}}}+\ln \left(\frac{\mu^{2}}{\mathbf{r}^{2}}\right)\right] .
$$

The total subtraction $I_{1}^{\text {cross }}+I_{2}^{\text {cross }}$ gives

$$
I_{1}^{\text {cross }}+I_{2}^{\text {cross }}=-\frac{i}{4 \pi^{2} \mathbf{r}^{2}}\left[\frac{1}{\epsilon_{\mathrm{IR}}}-\frac{1}{\epsilon_{\mathrm{UV}}}\right],
$$

so the final result for the crossed-box integral is

$$
I_{S}^{\text {cross }}=\tilde{I}_{S}^{\text {cross }}-I_{1}^{\text {cross }}-I_{2}^{\text {cross }}=-\frac{i}{4 \pi^{2} \mathbf{r}^{2}}\left[\frac{1}{\epsilon_{\mathrm{UV}}}+\ln \left(\frac{\mu^{2}}{\mathbf{r}^{2}}\right)\right] .
$$

The subtractions have converted the $1 / \epsilon_{\mathrm{IR}}$ divergence in $\tilde{I}_{S}^{\text {cross }}$ into a $1 / \epsilon_{\mathrm{UV}}$ ultraviolet divergence. In Ref. [14, 52], it was argued that $1 / \epsilon_{\mathrm{IR}}$ divergences in soft graphs should be converted to ultraviolet divergences by a pullup mechanism and included in the computation of anomalous dimensions. We see that the zero-bin subtraction automatically implements this conversion. An important feature for NRQCD is that the soft graph defined with the 


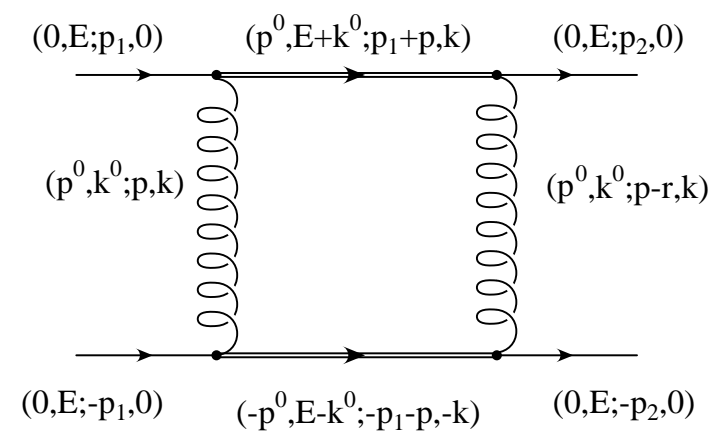

FIG. 8: Soft box graph in the effective theory. The zigzag lines are soft gluons, the double lines are soft quarks, and the single lines are potential quarks. For each line we show (label energy, residual energy; label momentum, residual momentum).

zero-bin subtraction is infrared finite, and has a well-defined renormalized value independent of any cancellation with usoft graphs.

A similar conversion from infrared to ultraviolet divergences also occurs for collinear graphs in $\mathrm{SCET}_{\mathrm{I}}$ as we show in section VI.

\section{B. NRQCD Box Graph}

The zero-bin subtraction has another important consequence - it gets rid of pinch singularities. Consider the soft box graph in NRQCD, shown in Fig. 8, and follow the same

procedure as for the crossed-box graph. The only difference from the crossed-box is the replacement

$$
\frac{1}{p^{0}+i 0^{+}} \frac{1}{p^{0}+i 0^{+}} \rightarrow \frac{1}{p^{0}+i 0^{+}} \frac{1}{-p^{0}+i 0^{+}}
$$

in Eqs. (18 21) due to the change in momentum routing through the antiquark line. The integrals analogous to those in Eqs. (19,21) are

$$
\begin{aligned}
\tilde{I}_{S}^{\text {box }} & =\int \frac{d^{D} p}{(2 \pi)^{D}} \frac{1}{p^{0}+i 0^{+}} \frac{1}{-p^{0}+i 0^{+}} \frac{1}{\left(p^{0}\right)^{2}-\mathbf{p}^{2}+i 0^{+}} \frac{1}{\left(p^{0}\right)^{2}-(\mathbf{p}-\mathbf{r})^{2}+i 0^{+}} \\
I_{1}^{\mathrm{box}} & =\int \frac{d^{D} p}{(2 \pi)^{D}} \frac{1}{p^{0}+i 0^{+}} \frac{1}{-p^{0}+i 0^{+}} \frac{1}{\left(p^{0}\right)^{2}-\mathbf{p}^{2}+i 0^{+}} \frac{1}{-(\mathbf{r})^{2}+i \epsilon} \\
I_{2}^{\text {box }} & =\int \frac{d^{D} p}{(2 \pi)^{D}} \frac{1}{p^{0}+i 0^{+}} \frac{1}{-p^{0}+i 0^{+}} \frac{1}{-\mathbf{r}^{2}+i 0^{+}} \frac{1}{\left(p^{0}\right)^{2}-(\mathbf{p}-\mathbf{r})^{2}+i 0^{+}}
\end{aligned}
$$

where the usoft subtractions $I_{1,2}^{\text {box }}$ are for $\left(p^{0}=0, \mathbf{p}=0\right),\left(p^{0}=0, \mathbf{p}=\mathbf{r}\right)$ respectively. In addition one also has a potential subtraction for $p^{0}=0$,

$$
I_{3}^{\text {box }}=\int \frac{d^{D} p}{(2 \pi)^{D}} \frac{1}{p^{0}+i 0^{+}} \frac{1}{-p^{0}+i 0^{+}} \frac{1}{-\mathbf{p}^{2}+i 0^{+}} \frac{1}{-(\mathbf{p}-\mathbf{r})^{2}+i 0^{+}} .
$$


Now this $p^{0}=0$ subtraction overlaps with the usoft subtractions, so we have to add back the double subtractions, the $\left(p^{0}=0\right)$ limit of $I_{1,2}^{\text {box }}$ :

$$
\begin{aligned}
I_{4}^{\text {box }} & =\int \frac{d^{D} p}{(2 \pi)^{D}} \frac{1}{p^{0}+i 0^{+}} \frac{1}{-p^{0}+i 0^{+}} \frac{1}{-\mathbf{p}^{2}+i 0^{+}} \frac{1}{-\mathbf{r}^{2}+i 0^{+}} \\
I_{5}^{\mathrm{box}} & =\int \frac{d^{D} p}{(2 \pi)^{D}} \frac{1}{p^{0}+i 0^{+}} \frac{1}{-p^{0}+i 0^{+}} \frac{1}{-\mathbf{r}^{2}+i 0^{+}} \frac{1}{-(\mathbf{p}-\mathbf{r})^{2}+i 0^{+}}
\end{aligned}
$$

The complete expression for the soft box graph is

$$
I_{S}^{\mathrm{box}}=\tilde{I}_{S}^{\mathrm{box}}-I_{1}^{\mathrm{box}}-I_{2}^{\mathrm{box}}-I_{3}^{\mathrm{box}}+I_{4}^{\mathrm{box}}+I_{5}^{\mathrm{box}}
$$

Both $\tilde{I}_{S}^{\text {box }}$ and $I_{3}^{\text {box }}$ have pinch singularities in the $p^{0}$ integral, from the poles at $p^{0}= \pm i 0^{+}$, and are ill-defined. However, for the result in the effective theory, we don't need the separate integrals, but only the difference $\tilde{I}_{S}^{\text {box }}-I_{3}^{\text {box }}$, which has no pinch. We have

$$
\begin{aligned}
\tilde{I}_{S}^{\text {box }}-I_{3}^{\text {box }}= & \int \frac{d^{D} p}{(2 \pi)^{D}} \frac{1}{p^{0}+i 0^{+}} \frac{1}{-p^{0}+i 0^{+}} \times \\
& \left\{\frac{1}{\left(p^{0}\right)^{2}-\mathbf{p}^{2}+i 0^{+}} \frac{1}{\left(p^{0}\right)^{2}-(\mathbf{p}-\mathbf{r})^{2}+i 0^{+}}-\frac{1}{\mathbf{p}^{2}+i 0^{+}} \frac{1}{(\mathbf{p}-\mathbf{r})^{2}+i 0^{+}}\right\}
\end{aligned}
$$

One can evaluate the $p^{0}$ integral in Eq. (30) using contour integration. The result is the same as doing Eq. (19) by contours and dropping the pinch pole at $p^{0}=0$, since the integrand of Eq. (30) has no $p^{0}$ pole and the subtraction term does not introduce new poles in $p^{0}$. This prescription for the soft box graph is what was used in Refs. [9, 11, 12, 42, 49, 60], but we now see how the effective theory automatically gives this result. ${ }^{10}$

The double subtractions $I_{4}^{\text {box }}$ and $I_{5}^{\text {box }}$ remove the pinch poles at $p^{0}=0$ for the subtractions $I_{1}^{\text {box }}$ and $I_{2}^{\text {box }}$ respectively, so that $I_{1}^{\text {box }}-I_{4}^{\text {box }}$ and $I_{2}^{\text {box }}-I_{5}^{\text {box }}$ are free of pinch singularities. This justifies ignoring the $p^{0}=0$ pole in the calculation of these integrals. The $I_{1,2}^{\text {box }}$ usoft subtractions convert the infrared divergences in $\tilde{I}_{S}^{\text {box }}$ into ultraviolet divergences, just as they did for the crossed-box. Prior to the subtraction the soft box integral in dimensional regularization is

$$
\tilde{I}_{S}^{\mathrm{box}}-I_{3}^{\mathrm{box}}=\frac{i}{4 \pi^{2} \mathbf{r}^{2}}\left[\frac{1}{\epsilon_{\mathrm{IR}}}+\ln \left(\frac{\mu^{2}}{\mathbf{r}^{\mathbf{2}}}\right)\right] .
$$

The total subtraction is

$$
I_{1}^{\mathrm{box}}-I_{4}^{\mathrm{box}}+I_{2}^{\mathrm{box}}-I_{5}^{\mathrm{box}}=\frac{i}{4 \pi^{2} \mathbf{r}^{2}}\left[\frac{1}{\epsilon_{\mathrm{IR}}}-\frac{1}{\epsilon_{\mathrm{UV}}}\right]
$$

$\overline{10}$ At one-loop there are many prescriptions that lead to the same result, that the pinch is dropped in the contour integration. Examples include split dimensional regularization and the principal value prescription. The formula in Eq. (17) can be applied at any order, and makes adopting a prescription moot. 
so the final result for the box integral is

$$
I_{S}^{\mathrm{box}}=\tilde{I}_{S}^{\mathrm{box}}-I_{1}^{\mathrm{box}}-I_{2}^{\mathrm{box}}-I_{3}^{\mathrm{box}}+I_{4}^{\mathrm{box}}+I_{5}^{\mathrm{box}}=\frac{i}{4 \pi^{2} \mathbf{r}^{2}}\left[\frac{1}{\epsilon_{\mathrm{UV}}}+\ln \left(\frac{\mu^{2}}{\mathbf{r}^{\mathbf{2}}}\right)\right] .
$$

The subtractions have converted the $1 / \epsilon_{\mathrm{IR}}$ divergence in $\tilde{I}_{S}^{\text {box }}$ into a $1 / \epsilon_{\mathrm{UV}}$ ultraviolet divergence just like the cross-box. The zero-bin subtraction has removed the infrared divergences and the pinch singularities, since these regions are properly taken care of by usoft and potential graphs, respectively. The properly defined soft box graph is infrared finite, and has no pinch singularity.

The use of Eq. (17) with the zero-bin subtraction works at higher orders as well. One can check explicitly that the subtractions remove the pinch singularities in the double box. If the loop momenta for the two single-box subgraphs are called $p$ and $\ell$, the subtracted double-box is given by subtracting the region where $p^{0}=0$ and where $\ell^{0}=0$, and adding back the region where $p^{0}=\ell^{0}=0$. This gives an expression for the double box which is free of both single and double pinch singularities. We have checked that the subtracted three gluon exchange graphs give the correct contribution to the two-loop static potential [61] in Feynman gauge.

The standard computation of the Coulomb potential at $\mathcal{O}\left(\alpha_{s}^{2}\right)$ in QCD is free of IR singularities for a different reason, because the IR divergences in the box and crossed-box cancel against the vertex and wavefunction diagrams. With the zero-bin subtractions, the scaleless soft vertex and wavefunction diagrams are set to zero, and the box and cross-box together with the non-Abelian vacuum polarization and Y-graphs give the complete IR finite answer. At this order there is also no overall usoft contribution to this four point function, and the same is true for the Coulomb potential at two loop order [61, 62]. At three loops the zero-bin subtraction removes the ADM singularity in the Coulomb potential [15, 63, 64]. A one-loop example at $\mathcal{O}\left(v^{2}\right)$ where the subtractions do not cancel in the sum of diagrams is discussed in the next section.

\section{Results for the Box and Crossed-Box at order $\mathcal{O}\left(v^{2}\right)$}

In this section, we study the soft box and crossed-box graphs to second order in the $v$ expansion, i.e. to the same order as the spin-orbit, Darwin and tensor-force contributions to the $Q \bar{Q}$ potential. At order $v^{2}$, the naive soft box and crossed-box have IR divergences and there are also non-trivial contributions from usoft diagrams. We will summarize results for these graphs to illustrate how the zero-bin subtractions work. This example also illustrates a case where $F_{j}^{\text {sub }}$ in Eq. (17) involves a series of terms.

Prior to any subtractions, the necessary diagrams are simply given by all quark-antiquark scattering diagrams that are derived using the HQET Lagrangian up to $1 / m^{2}$. The full integrands are lengthy and we refer the reader to Refs. [9, 42]. After using standard tricks 
to trivialize the numerator momenta we are left with the basic integrals

$$
J(\alpha, \beta)=\sum_{p \in Z^{c}} \int \frac{d^{D} k}{(2 \pi)^{D}} \frac{1}{\left(p^{0}+i 0^{+}\right)^{\alpha}\left(-p^{0}+i 0^{+}\right)^{\beta}} \frac{1}{\left[\left(p^{0}\right)^{2}-\mathbf{p}^{2}+i 0^{+}\right]\left[\left(p^{0}\right)^{2}-(\mathbf{p}-\mathbf{r})^{2}+i 0^{+}\right]},
$$

where $Z^{c}=\left\{p^{0} \neq 0, p^{\mu} \neq 0, p^{\mu} \neq(0, \mathbf{r})\right\}$. The subtractions that account for $p^{0} \neq 0$ and remove the pinch singularity from the first two denominators involve $\delta$ derivatives of the second two denominators where $\delta$ is the nearest integer $\leq(\alpha+\beta)$. For this particular computation this is equivalent to ignoring these poles in the contour integral.

As in the previous section the removal of the remaining constraints is similar, with or without the pinches, so we will consider the case $\beta=0$ for simplicity. The naive integral and its subtractions for $\left\{p^{\mu} \neq(0, \mathbf{r}), p^{\mu} \neq 0\right\}$ respectively are

$$
\begin{aligned}
\tilde{J}(\alpha, 0) & =\int \frac{d^{D} p}{(2 \pi)^{D}} \frac{1}{\left(p^{0}+i 0^{+}\right)^{\alpha}\left[\left(p^{0}\right)^{2}-\mathbf{p}^{2}+i 0^{+}\right]\left[\left(p^{0}\right)^{2}-(\mathbf{p}-\mathbf{r})^{2}+i 0^{+}\right]}, \\
J_{1}(\alpha, 0) & =\int \frac{d^{D} p}{(2 \pi)^{D}} \frac{1}{\left(p^{0}+i 0^{+}\right)^{\alpha}\left[\left(p^{0}\right)^{2}-(\mathbf{p}-\mathbf{r})^{2}+i 0^{+}\right]} \sum_{k=0}^{\alpha-2} \frac{[-2(\mathbf{p}-\mathbf{r}) \cdot \mathbf{r}]^{k}}{\left[-\mathbf{r}^{2}\right]^{k+1}}, \\
J_{2}(\alpha, 0) & =\int \frac{d^{D} p}{(2 \pi)^{D}} \frac{1}{\left(p^{0}+i 0^{+}\right)^{\alpha}\left[\left(p^{0}\right)^{2}-\mathbf{p}^{2}+i 0^{+}\right]} \sum_{k=0}^{\alpha-2} \frac{[-2 \mathbf{p} \cdot \mathbf{r}]^{k}}{\left[-\mathbf{r}^{2}\right]^{k+1}} .
\end{aligned}
$$

where in $J_{1}$ and $J_{2}$ we have dropped terms that are obviously zero. Note that here removing the zero-bin requires a series of subtractions obtained from expanding the naive integrand about the zero-bin values. By translation invariance in dimensional regularization the two subtraction integrals are equal, $J_{1}(\alpha, 0)=J_{2}(\alpha, 0)$.

For $\alpha=1$, the subtractions are zero, $J_{1}=J_{2}=0$, and $\tilde{J}(1,0)$ is finite. For any other odd $\alpha$, both the naive integral and subtractions give zero. For even $\alpha \leq 0, \tilde{J}(\alpha, 0)$ is UV divergent and the subtractions give zero. Finally for even $\alpha \geq 2$ the base integral is IR divergent and the subtractions convert this to a UV divergence for $J(\alpha, 0)=\tilde{J}(\alpha, 0)-J_{1}(\alpha, 0)-J_{2}(\alpha, 0)$. As an example, consider $\alpha=4$. The naive integral is

$$
\tilde{J}(4,0)=\frac{i}{16 \pi^{2}}\left(-\frac{16}{3 \mathbf{r}^{4}}\right)\left[\frac{1}{\epsilon_{\mathrm{IR}}}+\ln \left(\frac{\mu^{2}}{\mathbf{r}^{2}}\right)+2\right],
$$

and the subtractions $J_{1}=J_{2}$ are given by

$$
\begin{aligned}
J_{2}(4,0) & =\int \frac{d^{D} p}{(2 \pi)^{D}} \frac{1}{\left(p^{0}+i 0^{+}\right)^{4}\left[\left(p^{0}\right)^{2}-\mathbf{p}^{2}+i 0^{+}\right]}\left[-\frac{1}{\mathbf{r}^{2}}-\frac{2 \mathbf{p} \cdot \mathbf{r}}{\mathbf{r}^{4}}-\frac{4(\mathbf{p} \cdot \mathbf{r})^{2}}{\mathbf{r}^{6}}\right] \\
& =0+0+\frac{i}{16 \pi^{2}}\left(-\frac{16}{3 \mathbf{r}^{4}}\right)\left[\frac{1}{\epsilon_{\mathrm{IR}}}-\frac{1}{\epsilon_{\mathrm{UV}}}\right] .
\end{aligned}
$$

Thus the full integral is $J(4,0)=\tilde{J}(4,0)-2 J_{2}(4,0)$ giving

$$
J(4,0)=\frac{i}{16 \pi^{2}}\left(-\frac{16}{3 \mathbf{r}^{4}}\right)\left[\frac{1}{\epsilon_{\mathrm{UV}}}+\ln \left(\frac{\mu^{2}}{\mathbf{r}^{2}}\right)+2\right],
$$


which does not have an IR pole. The same is true for all even $\alpha \geq 2$.

Lets consider the sum of all order $v^{2}$ NRQCD soft exchange diagrams (boxes, cross-boxes, and triple gluon graphs), and the vertex and wavefunction graphs, all computed in Feynman gauge with equal mass quarks and antiquarks. Using the naive $\tilde{J}$ integrands we find ${ }^{11}$

$$
\begin{aligned}
\tilde{S}_{\text {exchange }}= & \frac{i \alpha_{s}^{2}}{m^{2}}\left\{\left[C_{1}(1 \otimes 1)-\frac{C_{d}}{4}\left(T^{A} \otimes T^{A}\right)\right]\left(\frac{1}{\epsilon_{\mathrm{UV}}}+\frac{4}{3 \epsilon_{\mathrm{IR}}}\right)\right. \\
& +C_{A}\left(T^{A} \otimes T^{A}\right)\left[\left(\frac{13}{4 \epsilon_{\mathrm{UV}}}+\frac{1}{3 \epsilon_{\mathrm{IR}}}\right)+\frac{\left(\mathbf{p}^{2}+\mathbf{p}^{\prime 2}\right)}{2 \mathbf{r}^{2}}\left(-\frac{5}{3 \epsilon_{\mathrm{UV}}}-\frac{14}{3 \epsilon_{\mathrm{IR}}}\right)\right) \\
& \left.\left.+\Lambda\left(\frac{1}{2 \epsilon_{\mathrm{UV}}}+\frac{3}{\epsilon_{\mathrm{IR}}}\right)+\mathbf{S}^{2}\left(-\frac{11}{18 \epsilon_{\mathrm{UV}}}+\frac{2}{3 \epsilon_{\mathrm{IR}}}\right)+\mathrm{T}\left(-\frac{1}{36 \epsilon_{\mathrm{UV}}}+\frac{1}{6 \epsilon_{\mathrm{IR}}}\right)\right]\right\}, \\
\tilde{S}_{\text {vertex+w.fn. }}= & \frac{i \alpha_{s}^{2}}{m^{2}}\left(T^{A} \otimes T^{A}\right)\left\{\frac{4}{3}\left(C_{F}-\frac{C_{A}}{2}\right)-2 C_{A}\left(\frac{\mathbf{p}^{2}+\mathbf{p}^{\prime 2}}{2 \mathbf{r}^{2}}-\frac{\mathbf{S}^{2}}{3}-\frac{3 \Lambda}{2}-\frac{\mathrm{T}}{12}\right)\right\} \\
& \times\left(\frac{1}{\epsilon_{\mathrm{UV}}}-\frac{1}{\epsilon_{\mathrm{IR}}}\right)
\end{aligned}
$$

for the pole structure. We used the notation in Ref. [9] where in $S U\left(N_{c}\right)$ the color coefficients $C_{1}=\left(N_{c}^{2}-1\right) /\left(4 N_{c}^{2}\right), C_{d}=N_{c}-4 / N_{c}, C_{A}=N_{C}, C_{F}=\left(N_{c}^{2}-1\right) /\left(2 N_{c}\right)$, there are two color structures $(1 \otimes 1)$ and $\left(T^{A} \otimes T^{A}\right)$, and $\Lambda, \mathbf{S}^{2}$, and $\mathrm{T}$ are spin and momentum dependent structures,

$$
\mathbf{S}=\frac{\sigma_{1}+\sigma_{2}}{2}, \quad \Lambda=-i \frac{\mathbf{S} \cdot\left(p^{\prime} \times p\right)}{\mathbf{r}^{2}}, \quad T=\sigma_{1} \cdot \sigma_{2}-\frac{3 \mathbf{r} \cdot \sigma_{1} \mathbf{r} \cdot \sigma_{2}}{\mathbf{r}^{2}} .
$$

The sum of diagrams with naive integrands is

$$
\begin{aligned}
\tilde{S}=\frac{i \alpha_{s}^{2}}{m^{2}}\left\{C_{1}(1 \otimes 1)\left(\frac{1}{\epsilon_{\mathrm{UV}}}+\frac{4}{3 \epsilon_{\mathrm{IR}}}\right)+\left(T^{A} \otimes T^{A}\right)\left[\frac{4 C_{F}}{3}\left(\frac{1}{\epsilon_{\mathrm{UV}}}-\frac{1}{\epsilon_{\mathrm{IR}}}\right)-C_{d}\left(\frac{1}{4 \epsilon_{\mathrm{UV}}}+\frac{1}{3 \epsilon_{\mathrm{IR}}}\right)\right]\right. \\
+C_{A}\left(T^{A} \otimes T^{A}\right)\left[\left(\frac{31}{12 \epsilon_{\mathrm{UV}}}+\frac{1}{\epsilon_{\mathrm{IR}}}\right)+\frac{\left(\mathbf{p}^{2}+\mathbf{p}^{\prime 2}\right)}{2 \mathbf{r}^{2}}\left(-\frac{11}{3 \epsilon_{\mathrm{UV}}}-\frac{8}{3 \epsilon_{\mathrm{IR}}}\right)\right) \\
\left.\left.+\Lambda\left(\frac{7}{2 \epsilon_{\mathrm{UV}}}\right)+\mathbf{S}^{2}\left(\frac{1}{18 \epsilon_{\mathrm{UV}}}\right)+\mathrm{T}\left(\frac{5}{36 \epsilon_{\mathrm{UV}}}\right)\right]\right\} .
\end{aligned}
$$

After subtracting the zero-bin contributions to get the proper integrals $J(\alpha, \beta)$ we find

$$
\begin{aligned}
S=\frac{i \alpha_{s}^{2}}{m^{2}}\left\{C_{1}(1 \otimes 1)\left(\frac{7}{3 \epsilon_{\mathrm{UV}}}\right)-C_{d}\left(T^{A} \otimes T^{A}\right)\left(\frac{7}{12 \epsilon_{\mathrm{UV}}}\right)+C_{A}\left(T^{A} \otimes T^{A}\right)\left[\left(\frac{43}{12 \epsilon_{\mathrm{UV}}}\right)\right.\right. \\
\left.\left.+\frac{\left(\mathbf{p}^{2}+\mathbf{p}^{\prime 2}\right)}{2 \mathbf{r}^{2}}\left(\frac{-19}{3 \epsilon_{\mathrm{UV}}}\right)+\Lambda\left(\frac{7}{2 \epsilon_{\mathrm{UV}}}\right)+\mathbf{S}^{2}\left(\frac{1}{18 \epsilon_{\mathrm{UV}}}\right)+\mathrm{T}\left(\frac{5}{36 \epsilon_{\mathrm{UV}}}\right)\right]\right\} .
\end{aligned}
$$

\footnotetext{
${ }^{11}$ For the purpose of this example we have set Wilson coefficients in the HQET Lagrangian to their tree level values. For the summation of logs in Refs. [42, 47, 53], their renormalization group evolution of course had to be kept.
} 
At $\mathcal{O}\left(v^{2}\right)$ there are also UV divergences from the usoft graphs which can be found from Ref. 42]. Using dimensional regularization for both the UV and the IR they give:

$$
U=\frac{i \alpha_{s}^{2}}{m^{2}}\left(\frac{1}{\epsilon_{\mathrm{UV}}}-\frac{1}{\epsilon_{\mathrm{IR}}}\right)\left\{\frac{4 C_{1}}{3}(1 \otimes 1)+\left[C_{A}-\frac{4 C_{F}}{3}-\frac{C_{d}}{3}-\frac{8 C_{A}}{3} \frac{\left(\mathbf{p}^{2}+\mathbf{p}^{\prime 2}\right)}{2 \mathbf{r}^{2}}\right]\left(T^{A} \otimes T^{A}\right)\right\} .
$$

We see explicitly that the usoft graphs have UV divergences which match up with the fake IR divergences from the unsubtracted soft computation in $\tilde{S}$. The true soft computation gives an IR finite result and the usoft contribution exactly matches the IR divergences in the full theory computation, see Ref. [9]. To interpret the UV divergence in Eq. (43) as occurring at the hard scale, it is crucial to make the zero-bin subtractions to avoid double counting in the soft region.

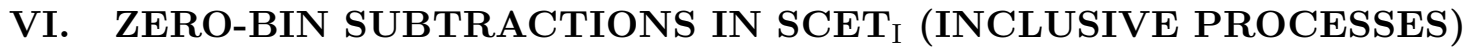

SCET is another theory with correlated scales, and with multiple fields for the same particle. As a result, one expects the zero-bin subtraction to also apply in this theory (yielding a pullup here too). In this part, we consider examples of the application of Eq. (17) to $\mathrm{SCET}_{\mathrm{I}}$ which has collinear fields appropriate for the description of perturbative energetic jets, and non-perturbative usoft fields. ${ }^{12}$ The new feature of $\mathrm{SCET}_{\mathrm{I}}$ is the appearance of double-logarithmic divergences at one-loop order. In the following discussion we show how the zero-bin subtraction works in this case.

We first consider the heavy to light vertex diagram in Fig. 9, which appears in processes such as the $b \rightarrow s \gamma$ transition magnetic moment operator needed to compute inclusive $B \rightarrow X_{s} \gamma$ decays. In section VIA we work on-shell with finite cutoffs and demonstrate that the infrared divergences of the full theory are reproduced in $\mathrm{SCET}_{\mathrm{I}}$. The subtraction from Eq. (17) in the collinear diagram is required for this to be true. To demonstrate that Eq. (17) is independent of the choice of ultraviolet and infrared regulator, in section VIB we consider the more standard choice of dimensional regularization with an offshellness infrared regulator, and explain how the zero-bin subtraction works for this case. In section VIO we treat the example of the current relevant for inclusive two-jet production where we have collinear fields in two directions, $n$ and $\bar{n}$, and usoft fields. The $\sum_{p \neq 0}$ are also important at tree level and for phase space integration as demonstrated by the $\gamma^{*} \rightarrow q \bar{q} g$ example that we take up in section $\nabla T D$.

\footnotetext{
12 These collinear modes are sometimes called hard-collinear, and the usoft modes are then called soft.
} 

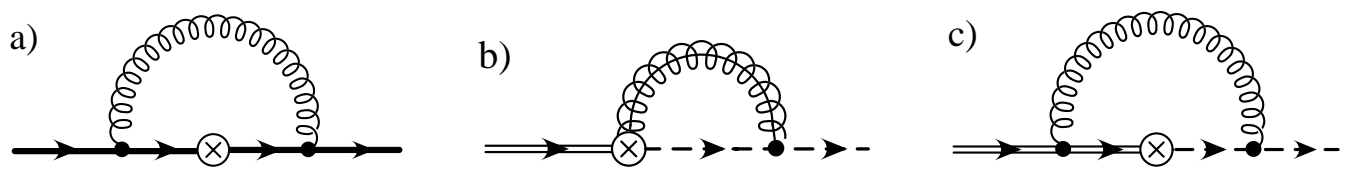

FIG. 9: Heavy-to-light vertex diagrams. a) full theory, b) SCET collinear graph, and c) SCET usoft graph.

\section{A. Onshell Integrals and a Cutoff Regulator for $B \rightarrow X_{s} \gamma$}

For $b \rightarrow s \gamma$ at lowest order in $\Lambda_{\mathrm{QCD}} / E_{\gamma}$ we have the SCET current [2]

$$
J^{(0)}=C(\omega)\left[\left(\bar{\xi}_{n} W\right)_{\omega} \Gamma h_{v}\right] .
$$

After making the decoupling field redefinition [4] on the collinear fields this becomes

$$
J^{\prime(0)}=C(\omega)\left[\left(\bar{\xi}_{n} W\right)_{\omega} \Gamma\left(Y^{\dagger} h_{v}\right)\right] .
$$

We start by making use of the current in Eq. (44) and will discuss the equivalence of using (45) at the end of this section. The incoming heavy quark momentum is $p_{b}^{\mu}=m v^{\mu}$ with $v^{2}=1$ and the outgoing light quark momentum is $p^{\mu}=p_{-} n^{\mu} / 2$ where $n^{2}=0$ and $p_{-}=\bar{n} \cdot p$. Both the incoming and outgoing quarks are taken onshell.

In this section we first demonstrate the effect of the pullup on collinear diagrams, prior to making a specific choice of regulator. We then use infrared cutoffs $\Omega_{\perp}$ and $\Omega_{-}$on $p_{\perp}$ and $p_{-}$in both the full and effective theories, so that the loop momenta are restricted to the region $q_{\perp}^{2} \geq \Omega_{\perp}^{2}$ and $q_{-}^{2} \geq \Omega_{-}^{2}$. For the usoft graphs, we use a ultraviolet cutoff $\Lambda_{-}$, while for the collinear graphs, we use an ultraviolet cutoff $\Lambda_{\perp}$. Hard cutoffs make the computation of anomalous dimensions more difficult and in more generic diagrams would require gauge violating (and power counting violating) counterterms to restore these symmetries. Our focus is on showing how the IR divergences are reproduced for a particular example where these problems do not occur, so for the purpose of this computation these issues are not a concern.

The part of the full theory diagram in Fig. 9a with the double logarithmic infrared divergence involves the integral

$$
I_{\text {full }}^{b \rightarrow s \gamma}=\int \frac{d^{D} q}{(2 \pi)^{D}} \frac{4 p_{b} \cdot p}{\left(q^{2}+i 0^{+}\right)\left(q^{2}+2 p_{b} \cdot q+i 0^{+}\right)\left(q^{2}+2 p \cdot q+i 0^{+}\right)},
$$

which is ultraviolet finite. Taking $p_{b}=m_{b}(n+\bar{n}) / 2$ and $0<p^{-}<m_{b}$, we use the identity $d^{D} q=d(n \cdot q) d(\bar{n} \cdot q) d^{n} q_{\perp} / 2$ to write the measure in light-cone variables, where here the exponent $n=D-2$ is the dimension of the $\perp$-space, not to be confused with the light-like 
vector $n^{\mu}$. Next we perform the $n \cdot q$ integral by contours. There are poles for

$$
\begin{array}{ll}
n \cdot q & =-\frac{q_{\perp}^{2}}{\bar{n} \cdot q}-i 0^{+} \operatorname{sign}(\bar{n} \cdot q), \quad n \cdot q=-\frac{\left(q_{\perp}^{2}+m_{b} \bar{n} \cdot q\right)}{\bar{n} \cdot q+m_{b}}-i 0^{+} \operatorname{sign}\left(\bar{n} \cdot q+m_{b}\right), \\
n \cdot q & =-\frac{q_{\perp}^{2}}{\bar{n} \cdot q+\bar{n} \cdot p}-i 0^{+} \operatorname{sign}(\bar{n} \cdot q+\bar{n} \cdot p),
\end{array}
$$

which gives three poles above the axis for $q^{-}<-m_{b}$, one moving below in the region $-m_{b}<q^{-}<-p_{-}$, two below and one above for $-p^{-}<q^{-}<0$, and all below for $q^{-}>0$. Thus only the middle two regions contribute. We will drop the integral over the interval $-m<\bar{n} \cdot q<-p_{-}$, since it is finite in the UV and IR. This gives

$$
I_{\text {full }}^{b \rightarrow s \gamma}=\frac{i}{2 \pi} \int_{-p_{-}}^{0} d \bar{n} \cdot q \frac{d^{n} q_{\perp}}{(2 \pi)^{n}} \frac{\bar{n} \cdot q}{\left[q_{\perp}^{2}+(\bar{n} \cdot q)^{2}\right]\left(q_{\perp}^{2}\right)}+\text { finite }
$$

The usoft graph in $\mathrm{SCET}_{\mathrm{I}}$ is shown in Fig. 9c and just has an integral over residual momentum. It is important to recall that the leading order SCET Lagrangian involves a momentum space multipole expansion for the residual momentum [2], so that only the residual $n \cdot k$ momentum appears in the collinear quark propagator. The integral is

$$
I_{\mathrm{us}}^{b \rightarrow s \gamma}=\int \frac{d^{D} k}{(2 \pi)^{D}} \frac{1}{\left(k^{2}+i 0^{+}\right)\left(v \cdot k+i 0^{+}\right)\left(n \cdot k+i 0^{+}\right)}=\frac{i}{2 \pi} \int_{-\infty}^{0} d \bar{n} \cdot k \frac{d^{n} k_{\perp}}{(2 \pi)^{n}} \frac{\bar{n} \cdot k}{\left[k_{\perp}^{2}+(\bar{n} \cdot k)^{2}\right]\left(k_{\perp}^{2}\right)} .
$$

For the collinear graph, Fig. 9b, we have the label loop momentum $\tilde{q}^{\mu}=\bar{n} \cdot q n^{\mu} / 2+q_{\perp}^{\mu}$ and residual loop momentum $q_{r}^{\mu}$, and we will denote $q^{\mu}=\tilde{q}^{\mu}+n \cdot q_{r} \bar{n}^{\mu} / 2$. The original integral is

$$
I_{\mathrm{C}}^{b \rightarrow s \gamma}=\sum_{\tilde{q} \neq 0, \tilde{q} \neq-\tilde{p}} \int \frac{d^{D} q_{r}}{(2 \pi)^{D}} \frac{2 \bar{n} \cdot(q+p)}{\left(\bar{n} \cdot q+i 0^{+}\right)\left(q^{2}+2 p \cdot q+i 0^{+}\right)\left(q^{2}+i 0^{+}\right)} .
$$

Eq. (17) is used to take into account the subtractions from the zero-bins. For $\tilde{q} \neq 0$ we examine the scaling $\tilde{q}^{\mu} \sim \lambda^{2}$, for which case the loop-measure scales as $\left[\lambda^{8}\right]$ and combines with the integrand to give: $\left[\lambda^{8}\right] /\left[\left(\lambda^{2}\right)\left(\lambda^{2}\right)\left(\lambda^{4}\right)\right] \sim \lambda^{0}$, so there is a non-trivial subtraction for this region. For $\tilde{q} \neq-\tilde{p}$ we examine $q^{-}+p^{-} \sim \lambda^{2}, q^{\perp} \sim \lambda^{2}$ and have: $\left[\lambda^{8}\right] /\left[\left(\lambda^{0}\right)\left(\lambda^{4}\right)\left(\lambda^{2}\right)\right] \sim \lambda^{2}$, so this zero-bin can be ignored. When we combine the sum over label momentum with the integral over residual momentum we get the naive result $\tilde{I}_{C}$ and a subtraction $I_{0}$ from Eq. (17):

$$
\begin{aligned}
\tilde{I}_{\mathrm{C}}^{b \rightarrow s \gamma} & =\int \frac{d^{D} q}{(2 \pi)^{D}} \frac{2 \bar{n} \cdot(q+p)}{\left(\bar{n} \cdot q+i 0^{+}\right)\left(q^{2}+2 p \cdot q+i 0^{+}\right)\left(q^{2}+i 0^{+}\right)}=\frac{i}{2 \pi} \int_{-p^{-}}^{0} d \bar{n} \cdot q \frac{d^{n} q_{\perp}}{(2 \pi)^{n}} \frac{\bar{n} \cdot(q+p)}{(\bar{n} \cdot q)(\bar{n} \cdot p)\left(q_{\perp}^{2}\right)} \\
I_{0}^{b \rightarrow s \gamma} & =\int \frac{d^{D} q}{(2 \pi)^{D}} \frac{2 \bar{n} \cdot p}{\left(\bar{n} \cdot q+i 0^{+}\right)\left(n \cdot q \bar{n} \cdot p+i 0^{+}\right)\left(q^{2}+i 0^{+}\right)}=\frac{i}{2 \pi} \int_{-\infty}^{0} d \bar{n} \cdot q \frac{d^{n} q_{\perp}}{(2 \pi)^{n}} \frac{\bar{n} \cdot p}{(\bar{n} \cdot q)(\bar{n} \cdot p)\left(q_{\perp}^{2}\right)},(51)
\end{aligned}
$$

Here the zero-bin subtraction $I_{0}^{b \rightarrow s \gamma}$ is obtained from the $q^{\mu} \rightarrow Q \lambda^{2}$ scaling limit of the $I_{C}^{b \rightarrow s \gamma}$ integrand and avoids double counting for the usoft region of momentum space. To double 
logarithmic accuracy the $\bar{n} \cdot q$ in the numerator of $\tilde{I}_{C}^{b \rightarrow s \gamma}$ can be dropped so

$$
I_{\mathrm{C}}^{b \rightarrow s \gamma}=\tilde{I}_{\mathrm{C}}^{b \rightarrow s \gamma}-I_{0}^{b \rightarrow s \gamma}=\frac{i}{2 \pi} \int_{-p^{-}}^{-\infty} d \bar{n} \cdot q \frac{d^{n} q_{\perp}}{(2 \pi)^{n}} \frac{1}{(\bar{n} \cdot q)\left(q_{\perp}^{2}\right)}+\ldots
$$

We see that the subtraction integral changes an infrared divergence in $I_{C}$ at $\bar{n} \cdot q=0$ into a ultraviolet divergence for $\bar{n} \cdot q \rightarrow-\infty$. Since we can see this at the level of the integrand it is obviously independent of the choice of ultraviolet and infrared regulators.

With the prescribed cutoff regulators and $\perp$-spacetime dimension $n=2$, these integrals can be evaluated to give

$$
\begin{aligned}
I_{\text {full }}^{b \rightarrow s \gamma} & =\frac{i}{8 \pi^{2}}\left[\operatorname{Li}_{2}\left(\frac{-\Omega_{\perp}^{2}}{\Omega_{-}^{2}}\right)+\ln \left(\frac{\Omega_{-}}{p^{-}}\right) \ln \left(\frac{\Omega_{-} p^{-}}{\Omega_{\perp}^{2}}\right)\right]+\ldots, \\
I_{\mathrm{us}}^{b \rightarrow s \gamma} & =\frac{i}{8 \pi^{2}}\left[\operatorname{Li}_{2}\left(\frac{-\Omega_{\perp}^{2}}{\Omega_{-}^{2}}\right)+\ln \left(\frac{\Omega_{-}}{\Lambda_{-}}\right) \ln \left(\frac{\Omega_{-} \Lambda_{-}}{\Omega_{\perp}^{2}}\right)\right], \\
\tilde{I}_{\mathrm{C}}^{b \rightarrow s \gamma} & =\frac{i}{8 \pi^{2}}\left[-\ln \left(\frac{\Omega_{\perp}^{2}}{\Lambda_{\perp}^{2}}\right) \ln \left(\frac{\Omega_{-}}{p^{-}}\right)\right]+\ldots, \\
I_{0}^{b \rightarrow s \gamma} & =\frac{i}{8 \pi^{2}}\left[-\ln \left(\frac{\Omega_{\perp}^{2}}{\Lambda_{\perp}^{2}}\right) \ln \left(\frac{\Omega_{-}}{\Lambda_{-}}\right)\right] .
\end{aligned}
$$

The full result for the collinear graph is therefore

$$
I_{\mathrm{C}}^{b \rightarrow s \gamma}=\tilde{I}_{\mathrm{C}}^{b \rightarrow s \gamma}-I_{0}^{b \rightarrow s \gamma}=\frac{i}{8 \pi^{2}}\left[-\ln \left(\frac{\Omega_{\perp}^{2}}{\Lambda_{\perp}^{2}}\right) \ln \left(\frac{\Lambda_{-}}{p^{-}}\right)\right]+\ldots,
$$

and we see that the zero-bin subtraction $I_{0}^{b \rightarrow s \gamma}$ has converted an IR divergence $\ln \left(\Omega_{-}\right)$for the $q^{-}$variable in $\tilde{I}_{C}^{b \rightarrow s \gamma}$ into a UV divergence, $\ln \left(\Lambda_{-}\right)$. The sum of the $\mathrm{SCET}_{\mathrm{I}}$ effective theory contributions gives

$$
\begin{aligned}
I_{\mathrm{us}}^{b \rightarrow s \gamma}+I_{\mathrm{C}}^{b \rightarrow s \gamma}= & \frac{i}{8 \pi^{2}}\left[\operatorname{Li}_{2}\left(\frac{-\Omega_{\perp}^{2}}{\Omega_{-}^{2}}\right)+\ln \left(\frac{\Omega_{-}}{p^{-}}\right) \ln \left(\frac{\Omega_{-} p^{-}}{\Omega_{\perp}^{2}}\right)+\ln ^{2}\left(\frac{\Lambda_{\perp}}{p^{-}}\right)-\ln ^{2}\left(\frac{\Lambda_{\perp}}{\Lambda_{-}}\right)\right] \\
& +\ldots
\end{aligned}
$$

The first two terms on the r.h.s. contain the infrared divergences and exactly reproduce these divergences in the full theory result $I_{\text {full }}^{b \rightarrow s \gamma}$. Furthermore, the last two terms in Eq. (55) depend only on the ultraviolet cutoffs and the large label momentum $p^{-}$and can be compensated by a counterterm for the current in $\mathrm{SCET}_{\mathrm{I}}$. If $I_{0}^{b \rightarrow s \gamma}$ in Eq. (54) had been left out, then we would not properly reproduce the IR divergences in the full theory result. Furthermore, without $I_{0}^{b \rightarrow s \gamma}$, the ultraviolet cutoff dependent term would have cross terms $\ln \left(\Lambda_{-}\right) \ln \left(\Omega_{\perp}^{2}\right)$ and $\ln \left(\Lambda_{\perp}^{2}\right) \ln \left(\Omega_{-}\right)$and it would not be possible to cancel the cutoff dependence by a counterterm independent of the IR regulator.

The above calculation was performed for the current $J^{(0)}$ in Eq. (44). Since our regulator leaves all external lines onshell we obtain exactly the same results if we had started with the current $J^{\prime(0)}$ in Eq. (45), which is obtained after making a field redefinition involving the 
Wilson line $Y$. Since we work onshell the two forms of the current are equivalent, and the Feynman rule from the Wilson line $Y$ give exactly the same integral in Eq. (49). Thus our implementation of a cutoff IR regulator does not destroy the eikonal factorization embodied by the field redefinitions involving the Wilson line $Y$. This property of the field theory is not maintained with the offshellness IR regulator which we consider in the next section. This should be considered as a fault of this IR regulator as pointed out in Ref. [65. In Ref. [65] an energy dependent gluon mass regulator was studied which also preserves the field redefinition. ${ }^{13}$

\section{B. Offshell Regulator with Dimensional Regularization for $B \rightarrow X_{s} \gamma$}

We now repeat the calculation of the effective theory diagrams in the previous section but keep $p^{2} \neq 0$ to regulate the infrared and use dimensional regularization for the ultraviolet, $D=4-2 \epsilon$. The full theory integral is

$$
I_{\text {full }}^{b \rightarrow s \gamma}=\int \frac{d^{D} q}{(2 \pi)^{D}} \frac{4 p_{b} \cdot p}{\left(q^{2}+i 0^{+}\right)\left(q^{2}+2 p_{b} \cdot q+i 0^{+}\right)\left[(q+p)^{2}+i 0^{+}\right]} .
$$

The SCET integrals are

$$
\begin{aligned}
I_{\mathrm{us}}^{b \rightarrow s \gamma} & =\int \frac{d^{D} k}{(2 \pi)^{D}} \frac{1}{\left(k^{2}+i 0^{+}\right)\left(v \cdot k+i 0^{+}\right)\left(n \cdot k+p^{2} / \bar{n} \cdot p+i 0^{+}\right)} \\
\tilde{I}_{\mathrm{C}}^{b \rightarrow s \gamma} & =\int \frac{d^{D} q}{(2 \pi)^{D}} \frac{2 \bar{n} \cdot(q+p)}{\left(\bar{n} \cdot q+i 0^{+}\right)\left[(q+p)^{2}+i 0^{+}\right]\left(q^{2}+i 0^{+}\right)}, \\
I_{0}^{b \rightarrow s \gamma} & =\int \frac{d^{D} q}{(2 \pi)^{D}} \frac{2 \bar{n} \cdot p}{\left(\bar{n} \cdot q+i 0^{+}\right)\left(n \cdot q \bar{n} \cdot p+p^{2}+i 0^{+}\right)\left(q^{2}+i 0^{+}\right)} .
\end{aligned}
$$

Again, one can see that as $\bar{n} \cdot q \rightarrow 0$ the difference $\tilde{I}_{C}^{b \rightarrow s \gamma}-I_{0}^{b \rightarrow s \gamma}$ does not have an infrared divergence from this region. However in $I_{C}$ alone, there is an infrared divergence from this region that is not regulated by $p^{2} \neq 0$. It is regulated by dimensional regularization, and so

${ }^{13}$ Ref. 65] also argued that the $\bar{n} \cdot k \rightarrow 0$ divergence must be treated as a UV in the EFT since it comes from angles opposite to the collinear direction. The renormalizability properties of field theory only appear for large momenta, and the zero-bin turns this divergence into a true UV divergence. One must be careful about the distinction between angles for particle and antiparticle poles when determining that the $\bar{n} \cdot k \rightarrow 0$ divergence is IR. 
contributes to the $1 / \epsilon$ singular terms. Evaluating the above integrals we find,

$$
\begin{aligned}
I_{\mathrm{full}}^{b \rightarrow s \gamma} & =-\frac{i}{16 \pi^{2}}\left[\ln ^{2}\left(\frac{-p^{2}}{[\bar{n} \cdot p]^{2}}\right)\right]+\ldots, \\
I_{\mathrm{us}}^{b \rightarrow s \gamma} & =-\frac{i}{16 \pi^{2}}\left[\frac{1}{\epsilon_{\mathrm{UV}}^{2}}+\frac{2}{\epsilon_{\mathrm{UV}}} \ln \left(\frac{\mu \bar{n} \cdot p}{-p^{2}}\right)+2 \ln ^{2}\left(\frac{\mu \bar{n} \cdot p}{-p^{2}}\right)\right]+\ldots, \\
\tilde{I}_{\mathrm{C}}^{b \rightarrow s \gamma} & =-\frac{i}{16 \pi^{2}}\left[-\frac{2}{\epsilon_{\mathrm{IR}} \epsilon_{\mathrm{UV}}}-\frac{2}{\epsilon_{\mathrm{IR}}} \ln \left(\frac{\mu^{2}}{-p^{2}}\right)-\ln ^{2}\left(\frac{\mu^{2}}{-p^{2}}\right)+\left(\frac{2}{\epsilon_{\mathrm{IR}}}-\frac{2}{\epsilon_{\mathrm{UV}}}\right) \ln \left(\frac{\mu}{\bar{n} \cdot p}\right)\right]+\ldots, \\
I_{0}^{b \rightarrow s \gamma} & =-\frac{i}{16 \pi^{2}}\left[\left(\frac{2}{\epsilon_{\mathrm{UV}}}-\frac{2}{\epsilon_{\mathrm{IR}}}\right)\left\{\frac{1}{\epsilon_{\mathrm{UV}}}+\ln \left(\frac{\mu^{2}}{-p^{2}}\right)-\ln \left(\frac{\mu}{\bar{n} \cdot p}\right)\right\}\right],
\end{aligned}
$$

where we have distinguished between ultraviolet and infrared divergences. Here we see that the zero-bin contribution $I_{0}^{b \rightarrow s \gamma}$ is responsible for canceling IR divergences in $\tilde{I}_{\mathrm{C}}^{b \rightarrow s \gamma}$ that were not regulated by the offshellness,

$$
I_{\mathrm{C}}^{b \rightarrow s \gamma}=\tilde{I}_{\mathrm{C}}^{b \rightarrow s \gamma}-I_{0}^{b \rightarrow s \gamma}=-\frac{i}{16 \pi^{2}}\left[-\frac{2}{\epsilon_{\mathrm{UV}}^{2}}-\frac{2}{\epsilon_{\mathrm{UV}}} \ln \left(\frac{\mu^{2}}{-p^{2}}\right)-\ln ^{2}\left(\frac{\mu^{2}}{-p^{2}}\right)\right]+\ldots
$$

Therefore the sum of the $\mathrm{SCET}_{\mathrm{I}}$ contributions gives

$$
I_{\mathrm{us}}^{b \rightarrow s \gamma}+I_{\mathrm{C}}^{b \rightarrow s \gamma}=-\frac{i}{16 \pi^{2}}\left[-\frac{1}{\epsilon_{\mathrm{UV}}^{2}}-\frac{2}{\epsilon_{\mathrm{UV}}} \ln \left(\frac{\mu}{\bar{n} \cdot p}\right)-2 \ln ^{2}\left(\frac{\mu}{\bar{n} \cdot p}\right)+\ln ^{2}\left(\frac{-p^{2}}{[\bar{n} \cdot p]^{2}}\right)\right]+\ldots
$$

The last term reproduces the infrared structure of the full theory result. The first two terms are canceled by a counterterm. The third term contributes a finite contribution to the hard Wilson coefficient of the heavy-to-light current in matching onto the full theory. Again we see that the contribution from $I_{0}^{b \rightarrow s \gamma}$ is necessary in order for the infrared divergences in the full and effective theories to match up. The ellipses in Eq. (60) denote 1/ $\epsilon$, single log, and finite terms that we have not bothered to display in the quoted results, but which have the same desired properties.

The computation of SCET anomalous dimensions in Ref. [2] and all subsequent papers used the entire $1 / \epsilon$ divergent terms in $\tilde{I}_{C}^{b \rightarrow s \gamma}$ to compute the anomalous dimension. As we have shown above, some of these divergences are, in fact, infrared divergences. The pullup mechanism is needed to convert these into ultraviolet divergences which can then be canceled by local counterterms in the effective theory, and so properly contribute to the anomalous dimension.

One can see the problem with having $\tilde{I}_{C}^{b \rightarrow s \gamma}$ and no subtraction term in another way. Consider adding a very small gluon mass to the collinear calculation, where $m^{2} \ll p^{2}$. The gluon mass can only affect the IR, and we should obtain the same form for the $1 / \epsilon_{\mathrm{UV}}$ divergences if we expand in $m^{2}$ before or after the integration. If we consider expanding after the integration, then performing the $q^{+}$integral by contours followed by the $q_{\perp}$ integration 
gives

$$
\begin{aligned}
& \tilde{I}_{\mathrm{C}}^{b \rightarrow s \gamma}=\frac{2 i \Gamma(\epsilon) \mu^{2 \epsilon}}{16 \pi^{2}} \int_{-p^{-}}^{0} \frac{d q^{-}}{q^{-}}\left(m^{2}+\frac{p^{2} q^{-}}{p^{-}}\right)^{-\epsilon}\left(1+\frac{q^{-}}{p^{-}}\right)^{-\epsilon} \\
& I_{0}^{b \rightarrow s \gamma}=\frac{2 i \Gamma(\epsilon) \mu^{2 \epsilon}}{16 \pi^{2}} \int_{-\infty}^{0} \frac{d q^{-}}{q^{-}}\left(m^{2}+\frac{p^{2} q^{-}}{p^{-}}\right)^{-\epsilon}
\end{aligned}
$$

Thus with $m^{2} \neq 0$ the IR singularity at $q^{-} \rightarrow 0$ is no longer regulated by dimensional regularization in $\tilde{I}_{C}^{b \rightarrow s \gamma}$ or $I_{0}^{b \rightarrow s \gamma}$, however the difference $I_{C}^{b \rightarrow s \gamma}=\tilde{I}_{C}^{b \rightarrow s \gamma}-I_{0}^{b \rightarrow s \gamma}$ remains well defined. Here the $1 / \epsilon$ terms in $\tilde{I}_{\mathrm{C}}^{b \rightarrow s \gamma}$ do not give the correct counterterm structure even if we set $\epsilon_{\mathrm{IR}}=\epsilon_{\mathrm{UV}}$. Letting $q^{-}=-x p^{-}$gives

$$
\begin{aligned}
I_{\mathrm{C}}^{b \rightarrow s \gamma} & =\frac{-2 i \Gamma(\epsilon) \mu^{2 \epsilon}}{16 \pi^{2}}\left[\int_{0}^{1} \frac{d x}{x}(1-x)^{-\epsilon}\left(m^{2}-x p^{2}\right)^{-\epsilon}-\int_{0}^{\infty} \frac{d x}{x}\left(m^{2}-x p^{2}\right)^{-\epsilon}\right] \\
& =\frac{-2 i \Gamma(\epsilon) \mu^{2 \epsilon}}{16 \pi^{2}}\left[\frac{\pi^{2} \epsilon}{6}-\int_{1}^{\infty} \frac{d x}{x}\left(m^{2}-x p^{2}\right)^{-\epsilon}\right] \\
& ==-\frac{i}{16 \pi^{2}}\left[-\frac{2}{\epsilon_{\mathrm{UV}}^{2}}-\frac{2}{\epsilon_{\mathrm{UV}}} \ln \left(\frac{\mu^{2}}{-p^{2}}\right)-\ln ^{2}\left(\frac{\mu^{2}}{-p^{2}}\right)-2 \mathrm{Li}_{2}\left(\frac{-m^{2}}{p^{2}}\right)+\ldots\right],
\end{aligned}
$$

and in the limit $m^{2} \ll p^{2}$ this reproduces Eq. (59). Thus with the zerobin subtractions the $1 / \epsilon_{\mathrm{UV}}$ divergences in the effective theory are independent of the choice of IR regulator.

Note that in this section it was crucial to use the current $J^{(0)}$ in order that taking $p^{2} \neq 0$ provides the same IR regulator in the full and effective theories. For the SCET current $J^{\prime(0)}$ this is no longer possible. Working with this current, only onshell IR regulators should be considered. This happens because the field redefinitions involving $Y$ 's modify the LO collinear Lagrangian, rather than just subleading terms, and are therefore sensitive to regulation of the propagator.

\section{Production of $n-\bar{n}$ jets}

As another example of the zero-bin subtractions in $\mathrm{SCET}_{\mathrm{I}}$ we consider the one-loop diagrams contributing to two jet production, $\gamma^{*} \rightarrow q \bar{q}$. The degrees of freedom required in $\mathrm{SCET}_{\mathrm{I}}$ are those pictured in Fig. Ha. In this case we have subtractions for both the $n$ collinear and $\bar{n}$-collinear fields, which ensure that they do not overlap with the usoft region. The leading order $\mathrm{SCET}_{\mathrm{I}}$ current is 66

$$
J^{(0)}=C\left(\omega, \omega^{\prime}\right)\left(\bar{\xi}_{n} W_{n}\right)_{\omega} \gamma^{\mu}\left(W_{\bar{n}}^{\dagger} \xi_{\bar{n}}\right)_{\omega^{\prime}}
$$

If we make the decoupling field redefinition [4] which encodes the eikonal coupling to all collinear quarks and gluons then $J^{(0)}$ becomes $J^{\prime(0)}=C\left(\omega, \omega^{\prime}\right)\left(\bar{\xi}_{n} W_{n}\right)_{\omega} Y_{n}^{\dagger} Y_{\bar{n}} \gamma^{\mu}\left(W_{\bar{n}}^{\dagger} \xi_{\bar{n}}\right)_{\omega^{\prime}}$. Below we work with the current $J^{(0)}$ since we will use an offshellness IR regulator.

The one-loop vertex graphs are shown in Fig. 10. Wavefunction graphs are not shown, but in Feynman gauge the collinear gluon wavefunction renormalization for a collinear quark is 

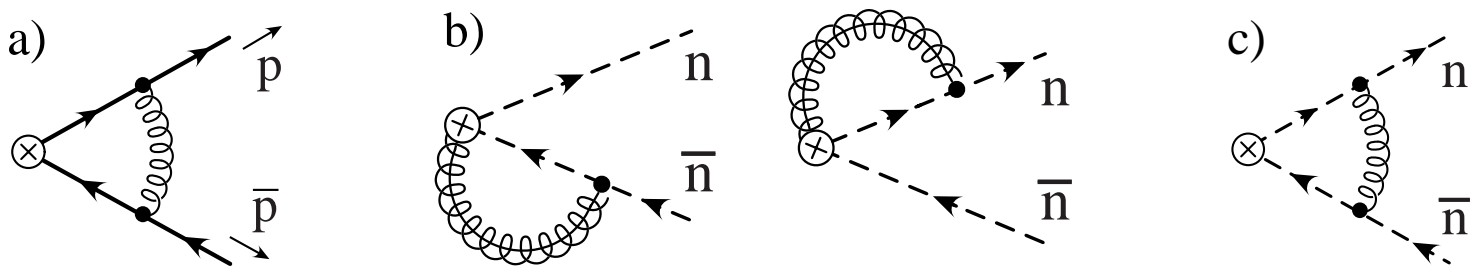

FIG. 10: Vertex diagrams for $n-\bar{n}$ production. a) full theory, b) SCET I collinear graphs, and c) $\mathrm{SCET}_{\mathrm{I}}$ usoft graph.

equal to the full theory result and the usoft gluon contribution vanishes. Working to double logarithmic order and breaking up the collinear terms into the naive result and subtractions the relevant integrals are

$$
\begin{aligned}
I_{\text {full }}^{\text {prod }} & =\int \frac{d^{D} q}{(2 \pi)^{D}} \frac{-4 p \cdot \bar{p}}{\left[q^{2}+i 0^{+}\right]\left[(q-\bar{p})^{2}+i 0^{+}\right)\left[(q+p)^{2}+i 0^{+}\right]}, \\
I_{\text {usoft }}^{\text {prod }} & =\int \frac{d^{D} q}{(2 \pi)^{D}} \frac{2(\bar{n} \cdot p)(-n \cdot \bar{p})}{\left[q^{2}+i 0^{+}\right]\left[\bar{n} \cdot p n \cdot q+p^{2}+i 0^{+}\right]\left[-n \cdot \bar{p} \bar{n} \cdot q+\bar{p}^{2}+i 0^{+}\right)}, \\
\tilde{I}_{n}^{\text {prod }} & =\int \frac{d^{D} q}{(2 \pi)^{D}} \frac{2 \bar{n} \cdot p}{\left[\bar{n} \cdot q+i 0^{+}\right]\left[(q+p)^{2}+i 0^{+}\right]\left[q^{2}+i 0^{+}\right]}, \\
I_{n 0}^{\text {prod }} & =\int \frac{d^{D} q}{(2 \pi)^{D}} \frac{2 \bar{n} \cdot p}{\left[\bar{n} \cdot q+i 0^{+}\right]\left[\bar{n} \cdot p n \cdot q+p^{2}+i 0^{+}\right]\left[q^{2}+i 0^{+}\right]}, \\
\tilde{I}_{\bar{n}}^{\text {prod }} & =\int \frac{d^{D} q}{(2 \pi)^{D}} \frac{2(-n \cdot \bar{p})}{\left[n \cdot q+i 0^{+}\right]\left[(q-\bar{p})^{2}+i 0^{+}\right]\left[q^{2}+i 0^{+}\right]}, \\
I_{\bar{n} 0}^{\text {prod }} & =\int \frac{d^{D} q}{(2 \pi)^{D}} \frac{2(-n \cdot \bar{p})}{\left[n \cdot q+i 0^{+}\right]\left[-n \cdot \bar{p} \bar{n} \cdot q+\bar{p}^{2}+i 0^{+}\right]\left[q^{2}+i 0^{+}\right]} .
\end{aligned}
$$

We have kept offshellnesses, $p^{2} \neq 0$ and $\bar{p}^{2} \neq 0$ to regulate the IR and will use dimensional regularization for the UV. Much like the heavy-to-light computation, this does not regulate all the IR divergences in the naive collinear integrands, $\tilde{I}_{n}^{\text {prod }}$ and $\tilde{I}_{\bar{n}}^{\text {prod }}$. Note that we took $\bar{n} \cdot(p+q) \rightarrow \bar{n} \cdot p$ in the numerator of the collinear graphs since we only examine the double logarithms and have made a corresponding approximation in $I_{\text {full }}^{\text {prod }}$. Evaluating the full theory integral we find ${ }^{14}$

$$
I_{\text {full }}^{\text {prod }}=-\frac{i}{8 \pi^{2}} \ln \left(\frac{p^{2}}{Q^{2}}\right) \ln \left(\frac{\bar{p}^{2}}{Q^{2}}\right)+\ldots
$$

The usoft loop graph in the effective theory gives

$$
I_{\mathrm{usoft}}^{\mathrm{prod}}=-\frac{i}{8 \pi^{2}}\left\{\frac{1}{\epsilon_{\mathrm{UV}}^{2}}+\frac{1}{\epsilon_{\mathrm{UV}}} \ln \left(\frac{\mu n \cdot \bar{p}}{\bar{p}^{2}}\right)+\frac{1}{\epsilon_{\mathrm{UV}}} \ln \left(\frac{\mu \bar{n} \cdot p}{-p^{2}}\right)+\frac{1}{2} \ln ^{2}\left[\frac{\bar{p}^{2}\left(-p^{2}\right)}{(\mu \bar{n} \cdot p)(\mu n \cdot \bar{p})}\right]+\ldots\right\} \cdot(60
$$

\footnotetext{
${ }^{14}$ In the computation of the cross-section in the full theory the $\ln \left(p^{2}\right)$ IR divergences are canceled by analogous IR divergences in the bremsstrahlung graphs.
} 
For the $n$-collinear naive integral and subtraction we find

$$
\begin{aligned}
& \tilde{I}_{n}^{\text {prod }}=-\frac{i}{16 \pi^{2}}\left[-\frac{2}{\epsilon_{\mathrm{IR}} \epsilon_{\mathrm{UV}}}-\frac{2}{\epsilon_{\mathrm{IR}}} \ln \left(\frac{\mu^{2}}{-p^{2}}\right)-\ln ^{2}\left(\frac{\mu^{2}}{-p^{2}}\right)+\left(\frac{2}{\epsilon_{\mathrm{IR}}}-\frac{2}{\epsilon_{\mathrm{UV}}}\right) \ln \left(\frac{\mu}{\bar{n} \cdot p}\right)\right]+\ldots, \\
& I_{n 0}^{\text {prod }}=-\frac{i}{16 \pi^{2}}\left[\left(\frac{2}{\epsilon_{\mathrm{UV}}}-\frac{2}{\epsilon_{\mathrm{IR}}}\right)\left\{\frac{1}{\epsilon_{\mathrm{UV}}}+\ln \left(\frac{\mu^{2}}{-p^{2}}\right)-\ln \left(\frac{\mu}{\bar{n} \cdot p}\right)\right\}\right]
\end{aligned}
$$

so the full $n$-collinear result is

$$
I_{n}^{\text {prod }}=\tilde{I}_{n}^{\text {prod }}-I_{n 0}^{\text {prod }}=-\frac{i}{8 \pi^{2}}\left[-\frac{1}{\epsilon_{\mathrm{UV}}^{2}}-\frac{1}{\epsilon_{\mathrm{UV}}} \ln \left(\frac{\mu^{2}}{-p^{2}}\right)-\frac{1}{2} \ln ^{2}\left(\frac{\mu^{2}}{-p^{2}}\right)\right]+\ldots
$$

Just as in the $b \rightarrow s \gamma$ example the subtraction terms remove the $1 / \epsilon_{\mathrm{IR}}$ poles, and the IR in the complete collinear integral is regulated by the offshellness. The ellipses denote $1 / \epsilon$, single log, and finite terms that we have not bothered to display in the quoted results. The results for the $\bar{n}$-collinear terms are similar

$$
\begin{aligned}
\tilde{I}_{\bar{n}}^{\text {prod }} & =-\frac{i}{16 \pi^{2}}\left[-\frac{2}{\epsilon_{\mathrm{IR}} \epsilon_{\mathrm{UV}}}-\frac{2}{\epsilon_{\mathrm{IR}}} \ln \left(\frac{\mu^{2}}{-\bar{p}^{2}}\right)-\ln ^{2}\left(\frac{\mu^{2}}{-\bar{p}^{2}}\right)+\left(\frac{2}{\epsilon_{\mathrm{IR}}}-\frac{2}{\epsilon_{\mathrm{UV}}}\right) \ln \left(\frac{\mu}{n \cdot \bar{p}}\right)\right]+\ldots, \\
I_{\bar{n} 0}^{\text {prod }} & =-\frac{i}{16 \pi^{2}}\left[\left(\frac{2}{\epsilon_{\mathrm{UV}}}-\frac{2}{\epsilon_{\mathrm{IR}}}\right)\left\{\frac{1}{\epsilon_{\mathrm{UV}}}+\ln \left(\frac{\mu^{2}}{-\bar{p}^{2}}\right)-\ln \left(\frac{\mu}{n \cdot \bar{p}}\right)\right\}\right] \\
I_{\bar{n}}^{\text {prod }} & =\tilde{I}_{\bar{n}}^{\text {prod }}-I_{\bar{n} 0}^{\text {prod }}=-\frac{i}{8 \pi^{2}}\left[-\frac{1}{\epsilon_{\mathrm{UV}}^{2}}-\frac{1}{\epsilon_{\mathrm{UV}}} \ln \left(\frac{\mu^{2}}{-\bar{p}^{2}}\right)-\frac{1}{2} \ln ^{2}\left(\frac{\mu^{2}}{-\bar{p}^{2}}\right)\right]+\ldots
\end{aligned}
$$

Adding up the SCET integrals, $I_{\text {scet }}^{\text {prod }}=I_{\text {usoft }}^{\text {prod }}+I_{\bar{n}}^{\text {prod }}+I_{n}^{\text {prod }}$, we find

$$
\begin{aligned}
I_{\mathrm{scet}}^{\mathrm{prod}} & =-\frac{i}{8 \pi^{2}}\left\{-\frac{1}{\epsilon_{\mathrm{UV}}^{2}}-\frac{1}{\epsilon_{\mathrm{UV}}} \ln \left(\frac{\mu^{2}}{-Q^{2}}\right)-\frac{1}{2} \ln ^{2}\left(\frac{\mu^{2}}{-\bar{p}^{2}}\right)-\frac{1}{2} \ln ^{2}\left(\frac{\mu^{2}}{-p^{2}}\right)+\frac{1}{2} \ln ^{2}\left(\frac{-\bar{p}^{2} p^{2}}{\mu^{2} Q^{2}}\right)\right\} \\
& =-\frac{i}{8 \pi^{2}}\left[-\frac{1}{\epsilon_{\mathrm{UV}}^{2}}-\frac{1}{\epsilon_{\mathrm{UV}}} \ln \left(\frac{\mu^{2}}{-Q^{2}}\right)-\frac{1}{2} \ln ^{2}\left(\frac{\mu^{2}}{-Q^{2}}\right)+\ln \left(\frac{\bar{p}^{2}}{Q^{2}}\right) \ln \left(\frac{p^{2}}{Q^{2}}\right)\right] .
\end{aligned}
$$

In the last line, the first two terms are removed by a counterterm in $\overline{\mathrm{MS}}$ and the third term contributes to the Wilson coefficient $C^{\text {prod }}$ in the one-loop matching, see Ref. [19]. The fourth term exactly reproduces the IR divergences in the full theory result.

In the above computation there was an interplay between the usoft loop and the $n$ and $\bar{n}$ collinear loops which combine to reproduce the IR of the full theory. The exact way in which these IR divergences combine depends on the choice of IR regulator as we saw in the $b \rightarrow s \gamma$ example. Again we see that the zero-bin subtractions are important to correctly reproduce the IR divergences once we distinguish between $\epsilon_{\mathrm{UV}}$ and $\epsilon_{\mathrm{IR}}$.

\section{D. $\gamma^{*} \rightarrow q \bar{q} g$ : The Zero-bin at Tree Level and for Phase Space Integrals}

In this section we show how the zero-bin is kept track of in tree level computations. One has to consider it even at tree level because in SCET multiple fields are present for 


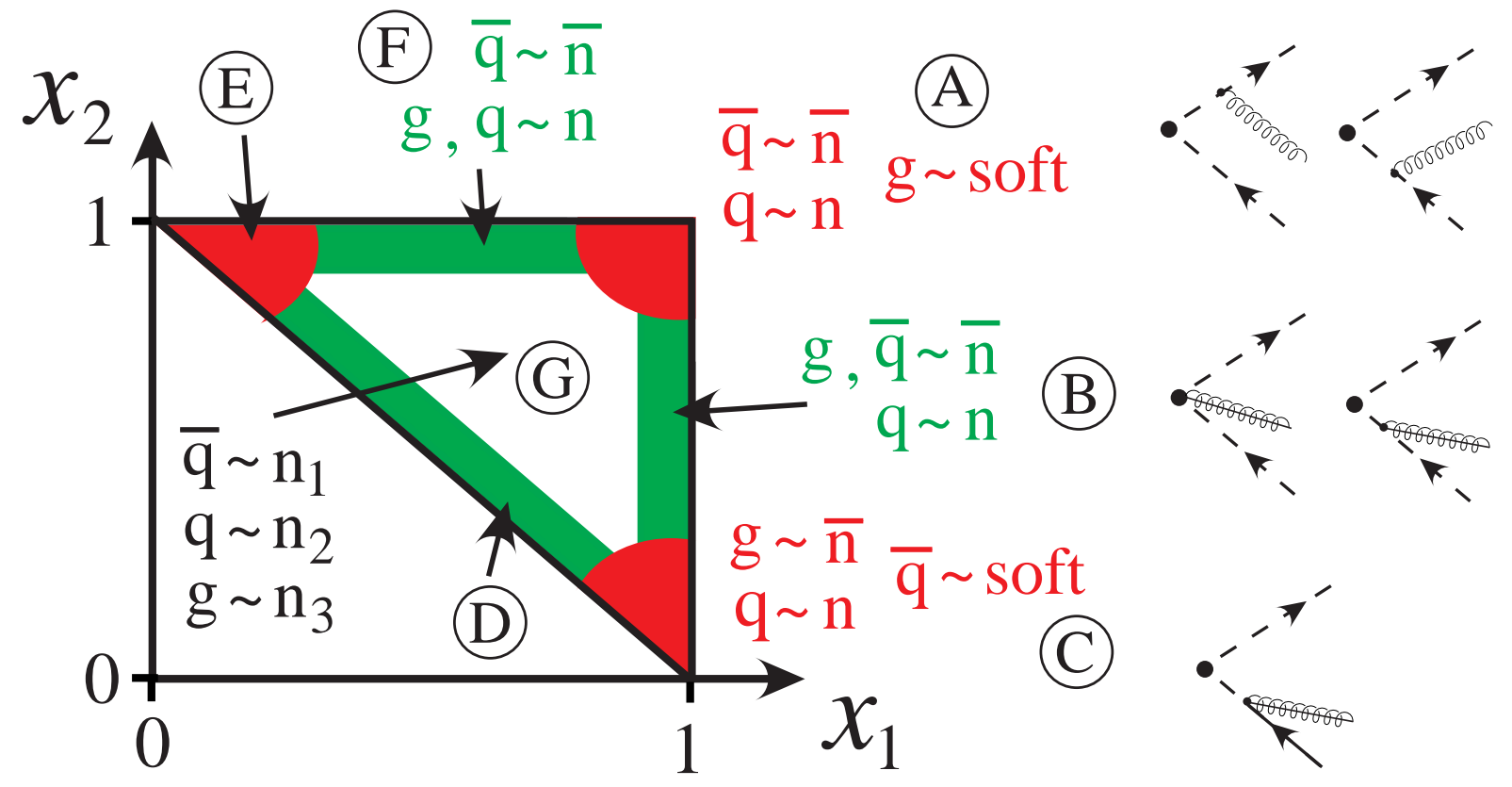

FIG. 11: Regions $\mathrm{R}=\mathrm{A}-\mathrm{G}$ for $\gamma^{*} \rightarrow q \bar{q} g$ where the particles become usoft and collinear. For regions A,B,C the graphs in SCET are shown. The soft regions are slightly exaggerated for visibility.

the same physical particle. We will demonstrate how double counting is avoided in fully differential cross sections and how the zero-bin subtraction affects phase space integrations. In the context of proving factorization in Drell-Yan, subtractions which avoid overcounting in phase space regions have been considered in Ref. [67].

Consider the high energy process $\gamma^{*}(q) \rightarrow q\left(p_{1}\right) \bar{q}\left(p_{2}\right) g\left(p_{3}\right)$ in the rest frame of the $\gamma^{*}$ with $q^{2}=Q^{2} \gg \Lambda_{\mathrm{QCD}}^{2}$. This is a basic ingredient in two-jet and three-jet production, which were considered in SCET in Refs. [68, 69, 70]. We take the full theory production current, $J=\bar{\psi} \gamma^{\mu} \psi$. The external lines have $p_{i}^{2}=0$ and we define dimensionless momentum fractions $x_{i}=2 q \cdot p_{i} / q^{2}$ so that momentum conservation reads $2=x_{1}+x_{2}+x_{3}$. Computing the phase space integrals in $(4-2 \epsilon)$-dimensions, the standard full theory cross section from the bremsstrahlung graphs is

$$
\frac{1}{\sigma_{0}} \frac{d \sigma_{\text {full }}}{d x_{1} d x_{2}}=\frac{C_{F} \alpha_{s}}{2 \pi} \frac{\mu^{2 \epsilon}}{q^{2 \epsilon}} \frac{1}{\Gamma(1-\epsilon)\left(x_{1}+x_{2}-1\right)^{\epsilon}} \frac{x_{1}^{2}+x_{2}^{2}-\epsilon\left(2-x_{1}-x_{2}\right)^{2}}{\left(1-x_{1}\right)^{1+\epsilon}\left(1-x_{2}\right)^{1+\epsilon}}
$$

where $\sigma_{0}$ is the Born cross section in dimensional regularization, $\sigma_{0}=\left(4 \pi \alpha^{2} / Q^{2}\right) \sum_{f} e_{f}^{2}+$ $\mathcal{O}(\epsilon)$ with a sum over the quark charges $e_{f}=2 / 3$ or $-1 / 3$.

Taking $x_{1}$ and $x_{2}$ as the independent variables we have the phase space shown in Fig. 11 . In SCET the different regions of the phase space plot are described by distinct EFT diagrams 
with the particles being created by either a collinear or usoft field. The relevant limits are

$$
\begin{array}{lll}
\mathrm{A}: x_{1,2} \rightarrow 1, & \mathrm{~B}: x_{1} \rightarrow 1, x_{2} \sim x, & \mathrm{C}: x_{1} \rightarrow 1, x_{2} \rightarrow 0, \\
\mathrm{D}: x_{1}+x_{2} \rightarrow 1, x_{1,2} \sim x, & \mathrm{E}: x_{1} \rightarrow 0, x_{2} \rightarrow 1, & \mathrm{~F}: x_{2} \rightarrow 1, x_{1} \sim x, \\
\mathrm{G}: x_{1}, x_{2}, x_{1}+x_{2} \sim x, & &
\end{array}
$$

where $x$ denotes generic values not near the two ends. In Fig. 11 the SCET graphs for regions A,B,C are shown. We will compute the sum of the square of SCET diagrams for each region and compare them with the full theory result for the double differential cross section and a single differential cross section. In the $\gamma^{*}$ rest frame with $2 q^{\mu}=Q n^{\mu}+Q \bar{n}^{\mu}$ there is still a rotational freedom in the $\perp$-plane which we can fix in performing the calculations. For $\mathrm{A}, \mathrm{B}, \mathrm{C}$ we take $p_{1}^{\perp}=0$, while for $\mathrm{E}, \mathrm{F}$ it is more convenient to take $p_{2}^{\perp}=0$.

Computing these SCET graphs with the phase space integrals in dimensional regularization we find cross sections in each of the regions $R$

$$
\frac{d \sigma_{R}}{\sigma_{0} d x_{1} d x_{2}}=\frac{C_{F} \alpha_{s}}{2 \pi} \frac{1}{\Gamma(1-\epsilon)\left(1-x_{1}\right)^{\epsilon}\left(1-x_{2}\right)^{\epsilon}\left(x_{1}+x_{2}-1\right)^{\epsilon}}\left|\mathcal{A}_{R}\left(x_{1}, x_{2}\right)\right|^{2} .
$$

Here $\mathcal{A}_{R}$ is the amplitude in region $R$ divided by $Z=2 g \sqrt{2(1-\epsilon)}$. From the tree level diagrams we find

$$
\begin{aligned}
\left.\left|\mathcal{A}_{A}\right|^{2}\right|_{\substack{x_{1} \neq 0 \\
x_{2} \neq 1-x_{1}}} & =\frac{1}{Z^{2}} \sum_{\text {spins }}\left|\bar{u}_{n}\left(p_{1}\right)\left\{\frac{-g n \cdot \varepsilon}{n \cdot p_{3}}+\frac{g \bar{n} \cdot \varepsilon}{\bar{n} \cdot p_{3}}\right\} \gamma_{\perp}^{\mu} T^{A} v_{\bar{n}}\left(p_{2}\right)\right|^{2} \\
& =\frac{2}{\left(1-x_{1}\right)\left(1-x_{2}\right)}, \\
\left.\left|\mathcal{A}_{B}\right|^{2}\right|_{\substack{x_{1} \neq 0 \\
x_{2} \neq 1 \\
x_{2} \neq 1-x_{1}}} & =\frac{1}{Z^{2}} \sum_{\text {spins }}\left|\bar{u}_{n}\left(p_{1}\right)\left\{\frac{-g n \cdot \varepsilon}{n \cdot p_{3}} \gamma_{\perp}^{\mu}+\frac{g n \cdot\left(p_{3}-p_{2}\right)}{\left(p_{3}-p_{2}\right)^{2}} \gamma_{\perp}^{\mu}\left(\bar{n} \cdot \varepsilon+\frac{\not \perp p h}{n \cdot p_{2}}\right)\right\} T^{A} v_{\bar{n}}\left(p_{2}\right)\right|^{2} \\
& =\frac{1+x_{2}^{2}}{\left(1-x_{1}\right)\left(1-x_{2}\right)}-\frac{\epsilon\left(1-x_{2}\right)}{\left(1-x_{1}\right)}, \\
\left.\left|\mathcal{A}_{C}\right|^{2}\right|_{\substack{x_{1} \neq 0 \\
x_{2} \neq 1}} & =\frac{1}{Z^{2}} \sum_{\text {spins }}\left|\bar{u}_{n}\left(p_{1}\right)\left\{\left(\frac{g \not h \not \perp}{2 \bar{n} \cdot p_{1}}\right) \gamma_{\perp}^{\mu}-\gamma_{\perp}^{\mu}\left(\frac{g \not h \notin \perp}{2 \bar{n} \cdot p_{1}}\right)\right\} T^{A} v\left(p_{2}\right)\right|^{2} \\
& =\frac{1-\epsilon}{\left(1-x_{1}\right)}
\end{aligned}
$$


and

$$
\begin{aligned}
& \left.\left|\mathcal{A}_{D}\right|^{2}\right|_{\substack{x_{1}+x_{2} \neq 2 \\
x_{1,2} \neq 0}}=\frac{1+\left(x_{1}-x_{2}\right)^{2}}{2 x_{1} x_{2}}+\mathcal{O}(\epsilon), \\
& \left.\left|\mathcal{A}_{E}\right|^{2}\right|_{\substack{x_{1} \neq 1-x_{2} \\
x_{2} \neq 0}}=\frac{1-\epsilon}{\left(1-x_{2}\right)}, \\
& \left.\left|\mathcal{A}_{F}\right|^{2}\right|_{\substack{x_{1} \neq 1-x_{2} \\
x_{1} \neq 1 \\
x_{2} \neq 0}}=\frac{1+x_{1}^{2}}{\left(1-x_{1}\right)\left(1-x_{2}\right)}-\frac{\epsilon\left(1-x_{1}\right)}{\left(1-x_{2}\right)}, \\
& \left.\left|\mathcal{A}_{G}\right|^{2}\right|_{\substack{x_{1}+x_{2} \neq 1 \\
x_{1,2} \neq 1}}=\frac{x_{1}^{2}+x_{2}^{2}}{\left(1-x_{1}\right)\left(1-x_{2}\right)}+\mathcal{O}(\epsilon) .
\end{aligned}
$$

Here $u_{n}, u_{\bar{n}}$, and $u$ are an $n$-collinear spinor, an $\bar{n}$-collinear spinor, and an usoft spinor respectively, all with relativistic normalization. For the regions A, B, C, we explicitly show the amplitudes that follow from the SCET Feynman diagrams in Fig. 11 and include $\epsilon$-dependent terms in the results. The amplitudes for A and B follow from the LO Lagrangians and LO SCET production current in Eq. (63). The result for $\mathrm{C}$ requires an insertion of the subleading Lagrangian $\mathcal{L}_{\xi q}^{(1)}=\bar{q}_{u s} W_{\bar{n}}^{\dagger} i g \not_{\perp}^{\bar{n}} \xi_{\bar{n}}+$ h.c. [71], where the field strength $i g \not_{\perp}^{\bar{n}}=\left[i \bar{n} \cdot D_{\bar{n}}^{c}, i D_{\perp}^{\bar{n}}\right]$. In Eq. (73) we have translated the zero-bin restrictions on the large momenta of collinear particles to restrictions on $x_{1}$ and $x_{2}$ as shown on the RHS of the equations.

It should be obvious from the form of $\mathcal{A}_{A}-\mathcal{A}_{G}$ that one can not simply add the SCET diagrams to reproduce the doubly differential cross section in Eq. (71). The point is that the effective theory results do not overlap, as made explicit by the sums which exclude the zero-bins, and constrain the valid region of phase space. Given an $x_{1}$ and $x_{2}$, only one of the effective theory expressions is relevant. It is straightforward to determine which one once we specify parametric definitions of the scaling limits in Eq. (72), and pick values for $x_{1}$ and $x_{2} .{ }^{15}$ The SCET diagrams in this region reproduce the full theory double differential cross section order by order in the expansion. Thus, it is crucial to take the zero-bin into account even at tree level in order to avoid double counting.

Often we would like to deal with a less differential cross section which involves integrating over kinematic variables. In this case we should implement the zero-bin subtractions in the phase space integrals using Eq. (17) to avoid double counting when combining regions. As an example of the zero-bin subtractions in phase space integrals we consider the $\gamma^{*} \rightarrow q \bar{q} g$ cross section $d \sigma / d x_{1}$ for fixed $x_{1}=1-\delta$ with $\delta \sim \lambda^{2}$ and nonzero. In the full theory the single differential cross section for $x_{1} \rightarrow 1$ is obtained by integrating Eq. (71) over $1-x_{1}<x_{2}<1$

15 The particles in the final state can be treated as observed, by a measurement of the final state. For each final state particle, one can assign a label $p$ and residual momentum $k$ as given by the binning of momentum space, and classify particles as collinear or usoft depending on whether $p \neq 0$. 
and expanding about $\delta$.

$$
\begin{aligned}
\left.\frac{1}{\sigma_{0}} \frac{d \sigma_{\mathrm{brem}}^{\text {full }}}{d x_{1}}\right|_{x_{1} \rightarrow 1} & =\frac{C_{F} \alpha_{s}}{\pi} \frac{\mu^{2 \epsilon}}{q^{2 \epsilon}} \frac{1}{\left(1-x_{1}\right)^{1+\epsilon} \Gamma(1-\epsilon)} K^{\text {full }}, \\
K^{\text {full }} & =\left[-\frac{1}{\epsilon_{\mathrm{IR}}}-\frac{3}{4}+\mathcal{O}(\delta)\right]
\end{aligned}
$$

where the IR divergence was regulated by dimensional regularization. To reproduce this result from the SCET computation requires adding contributions from regions $\mathrm{A}, \mathrm{B}$, and $\mathrm{C}$. By using Eq. (16) we can add these contributions and still integrate over the full phase space in $x_{2}$. For each region we can also expand the prefactor in Eq. (73) in the appropriate manner without effecting the LO results. Since $x_{1}$ is fixed we need not worry about subtractions involving this variable. Overlap occurs from $\left|\mathcal{A}_{B}\right|^{2}$ with region $\mathrm{C}$ if $x_{2}=0$ and with region A if $x_{2}=1$, so we find two zero-bin subtractions for these contributions. We multiply by $\left(1-x_{1}\right)^{1+\epsilon}$ to give the same normalization as Eq. (76). The regions with a soft particle are unsubtracted and give

$$
\begin{aligned}
& K^{A}=\left(1-x_{1}\right)^{1+\epsilon} \int_{1-x_{1}}^{1} d x_{2} \frac{2}{\left(1-x_{1}\right)^{1+\epsilon}\left(1-x_{2}\right)^{1+\epsilon}}=-\frac{1}{\epsilon_{\mathrm{IR}}}+\mathcal{O}(\delta), \\
& K^{C}=\left(1-x_{1}\right)^{1+\epsilon} \int_{1-x_{1}}^{1} d x_{2} \frac{1-\epsilon}{\left(1-x_{1}\right)^{1+\epsilon}\left(x_{1}+x_{2}-1\right)^{1+\epsilon}}=\frac{1}{2}+\mathcal{O}(\delta) .
\end{aligned}
$$

For region $\mathrm{B}$ with three collinear particles the naive contribution and its two subtractions $\left(x_{2}=1, x_{2}=0\right)$ are

$$
\begin{aligned}
& \tilde{K}^{B}=\left(1-x_{1}\right)^{1+\epsilon} \int_{1-x_{1}}^{1} d x_{2} \frac{\left[\left(1-x_{2}\right)^{2}(1-\epsilon)+2 x_{2}\right]}{\left(1-x_{1}\right)^{1+\epsilon}\left(1-x_{2}\right)^{1+\epsilon} x_{2}^{\epsilon}}=-\frac{1}{\epsilon_{\mathrm{IR}}}-\frac{3}{4}+\mathcal{O}(\delta), \\
& K_{1}^{B}=\left(1-x_{1}\right)^{1+\epsilon} \int_{1-x_{1}}^{1} d x_{2} \frac{2}{\left(1-x_{1}\right)^{1+\epsilon}\left(1-x_{2}\right)^{1+\epsilon}}=-\frac{1}{\epsilon_{\mathrm{IR}}}+\mathcal{O}(\delta) \\
& K_{2}^{B}=\left(1-x_{1}\right)^{1+\epsilon} \int_{1-x_{1}}^{1} d x_{2} \frac{1-\epsilon}{\left(1-x_{1}\right)^{1+\epsilon} x_{2}^{\epsilon}}=\frac{1}{2}+\mathcal{O}(\delta)
\end{aligned}
$$

For the collinear integral we therefore find

$$
K^{B}=\tilde{K}^{B}-K_{1}^{B}-K_{2}^{B}=-\frac{5}{4}+\mathcal{O}(\delta) .
$$

This result is IR finite as expected from the fact that the IR divergence comes from the soft region A in Fig. 11 and not from the collinear region B.

Adding the contributions from the three regions we find

$$
K^{A}+K^{B}+K^{C}=-\frac{1}{\epsilon_{\mathrm{IR}}}-\frac{3}{4},
$$

in agreement with the result for $K^{\text {full }}$ at this order. Thus with the zero-bin subtractions the sum of SCET diagrams reproduces the expected result for the $1 /\left(1-x_{1}\right)$ bremsstrahlung term in the cross section $d \sigma / d x_{1}$ as $x_{1} \rightarrow 1$. 

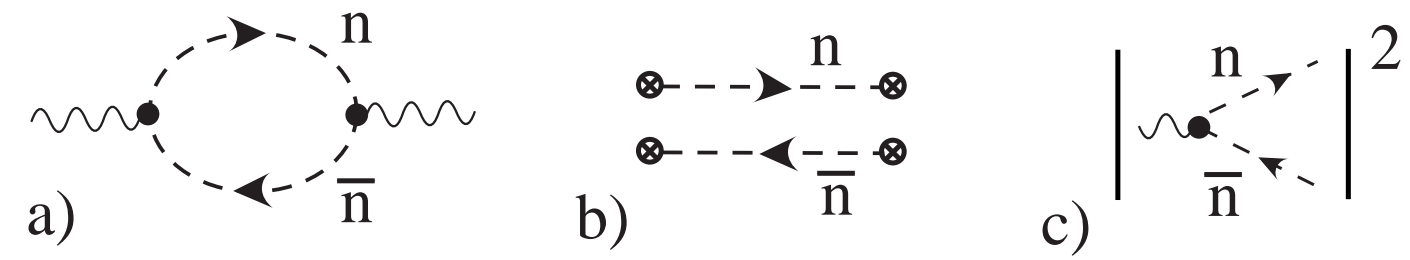

FIG. 12: Contraction of fields in the time ordered product of LO SCET currents, $T\left\{J^{(0)}, J^{(0) \dagger}\right\}$.

Finally, we briefly remark as to whether we could have turned the phase space computation into the imaginary part of a loop graph that we already know how to deal with from zero-bin examples in previous sections. One might think that the total cross section can be obtained by computing a forward scattering loop diagram in SCET and taking the imaginary part. However in some cases the optical theorem must be applied with care due to the momentum scaling of different types of SCET fields. A simple example is the Born cross section for $\gamma^{*} \rightarrow q \bar{q}$ which we can consider computing from $\operatorname{Im}\left[i \int d^{4} x \exp (-i q \cdot x)\left\langle 0\left|J(0) J^{\dagger}(x)\right| 0\right\rangle\right]$. In the full theory there is a contribution with hard loop momentum and the imaginary part contributes to the total- $\sigma$. In the SCET we are focusing on corners of phase space like back-to-back jets in the $n$ and $\bar{n}$ directions. The product of LO currents $J^{(0)}$ allows for a loop with usoft momentum, shown in Fig. 12a, without violating momentum conservation. However this graph evaluates to zero due to the multipole expansion on collinear lines,

$$
\int \frac{d^{D} k}{(2 \pi)^{D}} \frac{1}{(n \cdot(k+p))(\bar{n} \cdot(k+p)}=0 .
$$

The product of currents does give a nonzero contribution, just not from the imaginary part of this usoft loop in SCET. Instead, the SCET fields give the imaginary part of two propagators as in Fig. 12b, yielding an integrand for the phase space integral that is accurate in the desired phase space region for each line. Because the matrix element factorizes into a product of two matrix elements, Fig. 12 $\mathrm{b}$ is not a disconnected contribution that can be discarded. This reduces the problem back to squaring the current, as depicted in Fig. 12 c. It also gives a hint as to why the derivation of factorization formulas from SCET is more predictive than requiring a strict $\mathrm{OPE}$ in QCD, much as for diagrammatic factorization [38, 72, 73, 74].

\section{ZERO-BIN SUBTRACTIONS IN SCET II (EXCLUSIVE PROCESSES)}

In this section, we consider an SCET with degrees of freedom which are suitable for describing exclusive QCD processes with both energetic and soft hadrons. This theory is usually called $\mathrm{SCET}_{\mathrm{II}}$, and contains fields that describe nonperturbative collinear and soft momenta as pictured in the $p^{+}-p^{-}$plane shown in Fig. $13 .{ }^{16}$ It is also necessary to include

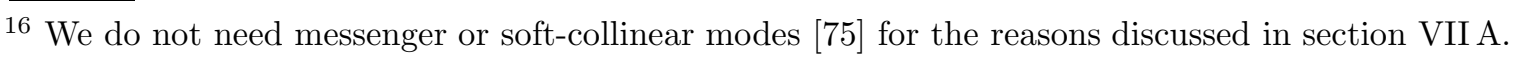




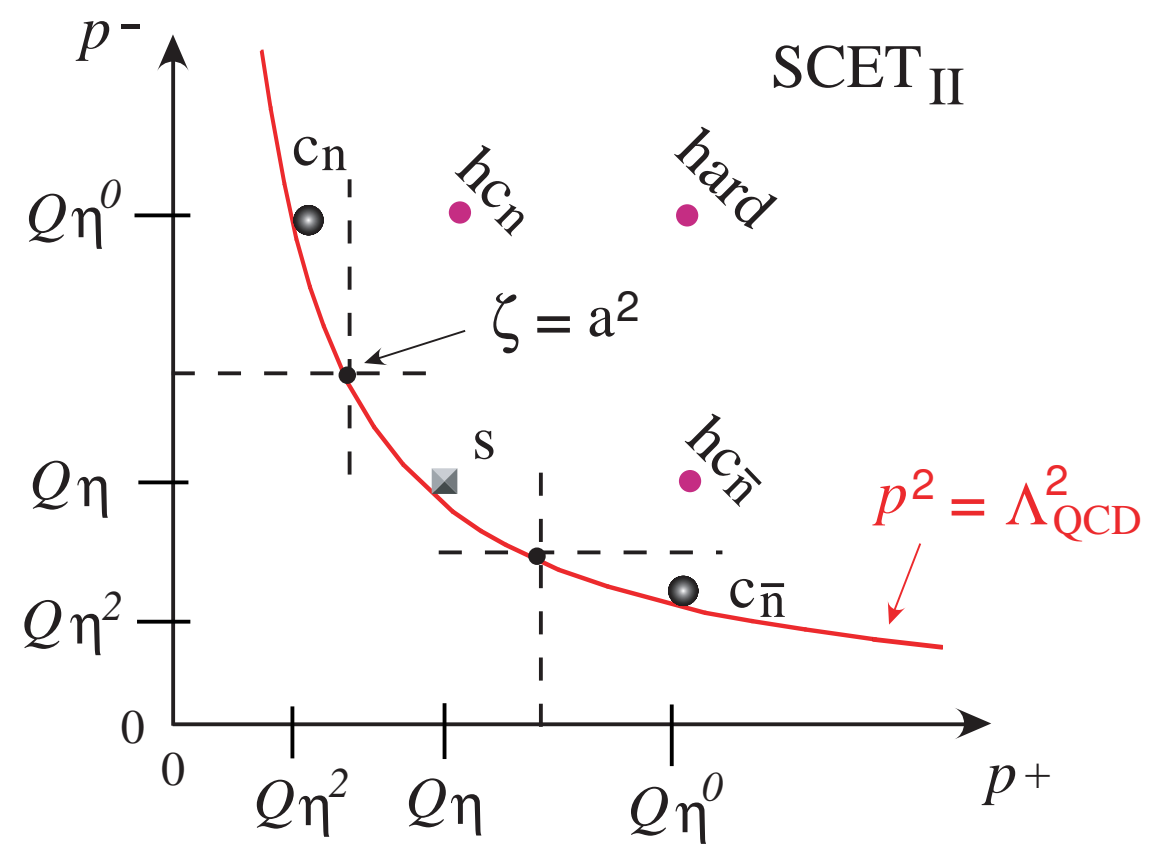

FIG. 13: Degrees of freedom and momentum regions for $\mathrm{SCET}_{\mathrm{II}}$ which describe non-perturbative fluctuations with $p^{2} \sim \Lambda_{\mathrm{QCD}}^{2}$. The modes include $n$-collinear $\left(c_{n}\right)$, soft $(s)$, and when applicable also $\bar{n}$-collinear $\left(c_{\bar{n}}\right)$. In perturbation theory these modes extend all the way in to zero-momenta. For QCD the solid (red) curve represents the region where infrared divergences are rendered finite by $\Lambda_{\mathrm{QCD}}$. Also shown (in pink) are three regions of perturbative momenta, two with hard-collinear momenta $\left(h c_{n}, h c_{\bar{n}}\right)$ and one where the momenta are hard.

these momentum regions when considering mixed inclusive and exclusive processes. We begin with a discussion of the ways in which SCET $_{\text {II }}$ differs from the SCET $_{I}$ and NRQCD examples discussed previously.

In perturbation theory with massless particles, physical IR divergences occur as $p^{2} \rightarrow$ 0 either with collinear scaling $\left(p^{+}, p^{-}\right) \sim Q\left(\eta^{2}, 1\right)$ or soft scaling $\left(p^{+}, p^{-}\right) \sim Q(\eta, \eta)$ for small dimensionless power counting parameters $\eta$ and a large momentum scale $Q$. This is well known from the study of the Landau equations and use of the Coleman-Norton theorem [72, 76]. In QCD, IR divergences either cancel between diagrams or are cutoff by the nonperturbative effects that generate confinement at a scale $p^{2} \sim \Lambda_{\mathrm{QCD}}^{2}$. In Fig. 13 we show the confinement scale by a red solid line. To formulate the power counting for these non-perturbative momenta, we take $\eta \sim \Lambda_{\mathrm{QCD}} / Q$. The collinear and soft fields represent distinct IR sectors as given by their momentum scaling, and together cover all approaches to the solid (red) curve in the $p^{+}-p^{-}$plane. ${ }^{17}$ The different sectors are separated by perturbative rapidity gaps.

${ }^{17}$ In this paper we do not consider processes which have important contributions from potential momenta for forward $n-\bar{n}$ scattering, $k^{+} k^{-} \ll k_{\perp}^{2}$, which are sometimes referred to as Glauber modes. 
The distinction between soft and collinear regions can be made with the variable

$$
\zeta_{p}=\frac{p^{-}}{p^{+}}
$$

which provides a measure along the solid red curve in Fig. 13. For the different regions we have:

$$
\begin{array}{rlrl}
n \text {-collinear: } & & \zeta_{p} & \sim \eta^{-2} \gg 1, \\
\text { soft: } & & \zeta_{p} \sim \eta^{0} \sim 1, \\
\bar{n} \text {-collinear: } & & \zeta_{p} & \sim \eta^{2} \ll 1 .
\end{array}
$$

Thus to avoid double counting, we must make sure that in the variable $\zeta_{p}$ the $n$-collinear mode does not double count the soft mode and vice-versa, and also that the $\bar{n}$-collinear mode does not double count the soft mode and vice-versa, etc. Note that the variable $\zeta_{p}$ provides a way of distinguishing the modes and at the same time allows us to maintain the boost-inversion symmetry [75, [77]. The boost-inversion symmetry allows one to swap the soft and $n$-collinear fields, etc. when setting up the modes for the description of a physical process.

As discussed in Ref. [4], momentum conservation strongly constrains the form of softcollinear interactions in $\mathrm{SCET}_{\mathrm{II}}$. Adding a soft mode $p_{s} \sim Q(\eta, \eta, \eta)$ to a collinear mode $p_{c} \sim Q\left(\eta^{2}, 1, \eta\right)$, which both have $p^{2} \sim Q^{2} \eta^{2}$, gives an offshell hard-collinear momentum $p_{h c}=p_{s}+p_{c}$ with $p_{h c}^{2} \sim Q^{2} \eta$. Thus all physical interaction Lagrangians and operators in $\mathrm{SCET}_{\text {II }}$ will have $\geq 2$ soft fields and $\geq 2$ collinear fields. As long as double counting (and divergent convolutions) are avoided, we can group like fields together in gauge invariant products to obtain factorized amplitudes at any order in the power expansion in $\eta$. In some cases, one can more directly prove that the convolution integrals converge [78, and for these cases it is less important to be careful about the zero-bins.

We will show that avoiding double counting in $\mathrm{SCET}_{\mathrm{II}}$ involves zero-bin subtractions similar to the previous sections, with the added complication associated with ensuring that regions in $\zeta_{p}$ are treated correctly. Due to UV divergences in rapidity, this requires a regularization method. It also requires extra renormalization parameters for the insertion of any operator that connects soft and collinear fields, which we denote by $\mu_{+}$and $\mu_{-}{ }^{18} \mathrm{In}$ dimensional regularization, the parts of the $\mathrm{SCET}_{\mathrm{II}}$ action that are purely soft or purely collinear have the standard $\mu^{2 \epsilon}$ multiplying couplings, so all factors of $\alpha_{s}$ are $\alpha_{s}(\mu)$. The factors of $\mu_{+}$and $\mu_{-}$only occur from mixed soft-collinear operators. We will show below how $\mu_{ \pm}$appear in dimensional regularization, and also with a cutoff regulator. The $\mu_{+}$and $\mu_{-}$parameters are tied together with the usual $\mu$ by the dynamics of factorization, which,

${ }^{18}$ For typographical convenience we use $\mu_{-}$and $\mu_{+}$although superscripts would be more appropriate. 
independent of the UV and IR regulators, gives

$$
\mu^{2}=\mu_{+} \mu_{-}
$$

Under an RPI-III transformation on the basis vectors, $n \rightarrow e^{\alpha} n$ and $\bar{n} \rightarrow e^{-\alpha} \bar{n}$ [79 (a longitudinal boost on coordinates and fields), $\mu_{+}$behaves like a momentum $p^{+}=n \cdot p$ and $\mu_{-}$behaves like $p^{-}=\bar{n} \cdot p$. Furthermore $\zeta_{p}$ scales under a RPI-III transformation. These boosts correspond to a universal shift of all degrees of freedom along the solid red curve in Fig. 13, and thus do not change the fact that having distinguished between modes using $\zeta_{p}$ in one frame we also avoid double counting in any other frame. For a process with only soft and $n$-collinear modes, the boost-inversion symmetry allows us to interchange the role of these modes [75]. In Fig. 13, we boost to lower $p^{-}$and increase $p^{+}$, so that the $c_{n}$ overlaps the $s$, and the $s$ overlaps the $c_{\bar{n}}$. We then switch our definition of plus and minus, $p^{+} \leftrightarrow p^{-}$, with the overall outcome that $c_{n} \leftrightarrow s$. Differentiating between modes using the variable $\zeta_{p}$ keeps them distinct throughout this process.

The basic structure that we have in mind for a factorization formula in $\mathrm{SCET}_{\mathrm{II}}$ is

$$
\int d k^{+} d k^{-} d p^{-} d p^{+} J\left(k^{ \pm}, p^{ \pm}, \mu_{ \pm}, \mu_{ \pm}^{\prime}\right) \phi_{n}\left(p^{-}, \mu_{-}, \mu^{2}\right) \phi_{s}\left(k^{+}, k^{-}, \mu_{+}, \mu_{-}^{\prime}\right) \phi_{\bar{n}}\left(p^{+}, \mu_{+}^{\prime}, \mu^{2}\right),
$$

where $J$ contains perturbative contributions from both hard-collinear and hard momenta (as shown by the solid pink dots in Fig. 13). For cases where only the $n$-collinear and soft modes are relevant, we have the slightly simpler form

$$
\int d k^{+} d p^{-} J\left(k^{+}, p^{-}, \mu_{+}, \mu_{-}\right) \phi_{n}\left(p^{-}, \mu_{-}, \mu^{2}\right) \phi_{s}\left(k^{+}, \mu_{+}, \mu^{2}\right)
$$

with $J$ purely hard-collinear. If only the $n$-collinear and $\bar{n}$-collinear modes are relevant, we have

$$
\int d k^{+} d p^{-} J\left(p^{+}, p^{-}, \mu_{-}, \mu_{+}^{\prime}\right) \phi_{n}\left(p^{-}, \mu_{-}, \mu^{2}\right) \phi_{\bar{n}}\left(p^{+}, \mu_{+}^{\prime}, \mu^{2}\right)
$$

with $J$ having hard momenta. The idea is that due to the separation of degrees of freedom in rapidity space the distributions can depend on $\mu_{ \pm}$. This dependence is similar to that for fragmentation functions in Ref. [33], 34]. The meaning of the $\mu_{+}$and $\mu_{-}$variables in the distribution functions is described further below in section VII below Eq. (129). The presence of the $\mu_{ \pm}$parameters allows us to formulate the non-perturbative matrix elements that give $\phi_{n}$ and $\phi_{s}$ as boost invariant objects. This evades an argument made in Ref. [77] that no IR regulator will allow a boost invariant factorization of soft and collinear modes in SCET $_{\text {II }}$. Our proposed factorization formula differs from the conclusion of non-factorization in Refs. [77, 78, 80]. The effects due to $\mu_{-}$and $\mu_{+}$are actually not IR sensitive: they denote a choice we have to distinguish the IR regions. They behave like the dimensional regularization parameter $\mu$ in that we can compute the dependence on these parameters in perturbation theory because of the large rapidity gaps. 
The soft and collinear modes in $\mathrm{SCET}_{\text {II }}$ generate the physical hadron states in the effective theory, with each mode generating the physical states in its sector. The two sectors are separated by a perturbatively large rapidity gap, so we do not need to consider hadrons made of both modes. Since near the mass-shell $p^{+} \sim p_{\perp}^{2} / p^{-}$, the rapidity scaling in Eq. (83) gives gaps in $p_{\perp} / E$ of spacing $\Lambda_{\mathrm{QCD}} / Q$. If we try to generate a hadron with an interpolating field built from soft and collinear fields, such as one collinear antiquark and one soft quark, then there are no physical non-perturbative poles by momentum conservation. Thus, the Hilbert space of states in the soft and collinear sectors are individually complete.

In section VII A we begin by discussing a loop integral in $\mathrm{SCET}_{\text {II }}$ taking into account the zero-bins. Our first one-loop example uses a hard cutoff regulator, in subsection VIIA 1. In section VIIB we formulate the separation of soft and collinear modes using dimensional regularization, and repeat the one-loop example in subsection VIIB1. In section VIIC we give a general discussion on how the zero-bin subtractions work on singular hard kernels to give what we call $\varnothing$-distributions (the complete definition can be found in this section). In section VIID we apply this formalism to obtain a result for the $\gamma^{*} \rho \rightarrow \pi$ form factor at large $Q^{2}$ which is free from convolution endpoint singularities. In section VIIE we discuss the so-called "soft" form factor for $B \rightarrow \pi$ transitions, $\zeta^{B \pi}(E)$, to argue that $\mathrm{SCET}_{\text {II }}$ yields a result in terms of individual $B$ and $\pi$ distribution functions.

\section{A. A SCET $\mathrm{II}$ Loop Integral with Subtractions}

As our first $\mathrm{SCET}_{\mathrm{II}}$ example, we consider a one-loop integral for the process " $B \rightarrow \gamma \ell \bar{\nu}$ " with $E_{\gamma} \gg \Lambda_{\mathrm{QCD}}$ but using scalar quarks and gluons. The LO factorization formula for the full QCD process was considered in Refs. [81, 82, 83, 84] using SCET. It involves $n$-hardcollinear fields and soft fields, but does not suffer from the subtleties in $\mathrm{SCET}_{\mathrm{II}}$ we wish to address. The toy example with scalars was considered in Ref. [78], where it was pointed out that this process with scalar quarks does not factor (naively) into a product of scalar and collinear terms, due to endpoint divergences which connect the soft and collinear matrix elements. This issue only shows up at subleading order for fermions.

In Ref. [77, 80] it was independently concluded that the convolution divergences encountered in these situations spoil factorization. The analysis was based on a different IR regulator, implemented with an offshellness, and adding to $\mathrm{SCET}_{\mathrm{II}}$ a so-called messenger or soft-collinear IR regulator mode which has $p^{2} \sim \Lambda_{\mathrm{QCD}}^{3} / Q$. Any long distance colored interaction in $\mathrm{SCET}_{\mathrm{II}}$ would violate confinement in $\mathrm{QCD}$ and therefore be forbidden, but in perturbation theory one is free to introduce modes with $p^{2}$ below $\Lambda_{\mathrm{QCD}}^{2}$ if they facilitate the regulation of IR divergences. ${ }^{19}$ The fact that messenger modes should be considered as

${ }^{19}$ In particular, any mode that would leave both soft and collinear modes onshell must have $p^{2} \ll \Lambda_{\mathrm{QCD}}^{2}$ by a parametric amount [75]. In QCD a physical IR cutoff is provided by confinement which eliminates any 
part of the IR regulator was discussed in Ref. [65], where it was shown that they are absent with an energy dependent gluon mass IR regulator, but that a common dependence on this regulator still appears in the soft and collinear matrix elements. ${ }^{20}$ Messenger modes were also absent with the calculations using analytic regulators in Ref. [78].

With our definition of modes in $\mathrm{SCET}_{\mathrm{II}}$ in Fig. 13, messenger modes are not needed. If,

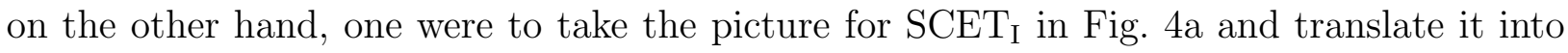
modes for $\mathrm{SCET}_{\mathrm{II}}$ in a one-to-one correspondence, then the usoft mode in $\mathrm{SCET}_{\mathrm{I}}$ becomes a messenger mode for $\mathrm{SCET}_{\mathrm{II}}$ [75]. So the $\mathrm{SCET}_{\mathrm{I}}$ mode decomposition seems to want a messenger mode in $\mathrm{SCET}_{\mathrm{II}}$. The fault here is with the translation, which required both a boost and a scale transformation. There is nothing wrong with the boost, but QCD is not scale invariant. Due to the presence of $\Lambda_{\mathrm{QCD}}$, translating the IR tiling of modes in $\mathrm{SCET}_{\mathrm{I}}$ into a tiling of IR modes for $\mathrm{SCET}_{\mathrm{II}}$, gives modes which hide the physical situation, in particular the perturbative split of the hadronic physics in rapidity space. In our definition of $\mathrm{SCET}_{\mathrm{II}}$ the IR regions that show up in perturbation theory and were described by the messenger mode in Ref. [75] are absorbed into the soft and collinear fields.

In considering scalar " $B \rightarrow \gamma \ell \bar{\nu}$ " we are really treating a subleading contribution to the physical process with fermions where the photon comes from fragmentation of $q \bar{q} \rightarrow \gamma$ or from a subleading contribution to the direct $\gamma$-production. In the fragmentation case we will have a non-perturbative soft distribution associated with the initial state $B, \phi_{s}\left(k^{+}\right)$, and a non-perturbative collinear distribution for the $\gamma$ associated with the fragmentation, $\phi_{n}\left(p^{-}\right)$. To factorize this physical process we must consider the imaginary part of the forward scattering, since due to the probabilistic interpretation for the fragmentation function, there will be a factorization formula for the decay rate but not for the amplitude. Examples of one loop diagrams in the full theory and $\mathrm{SCET}_{\mathrm{II}}$ are shown in Fig. 14. The $\mathrm{SCET}_{\text {II }}$ graphs in Fig. 14b correspond to direct production, while those in Fig. 14c are fragmentation. The $\mathrm{SCET}_{\text {II }}$ factorization allows these two effects to be distinguished.

In the full scalar theory we have a charged scalar "b-quark" with field $\phi^{b}$, charged light scalar "u-quark", $\phi$, and neutral "gluon" and "photon" fields $\phi^{g}$ and $\phi^{\gamma}$ respectively. The "weak" current and interaction terms are

$$
J_{\text {full }}^{\text {weak }}=G \phi^{\dagger} \phi^{b}, \quad \quad \mathcal{L}_{\text {full }}^{\text {int }}=g \phi^{g} \phi^{\dagger} \phi+e \phi^{\gamma} \phi^{\dagger} \phi,
$$

such modes 85] as they would physically correspond to colored degrees of freedom propagating between color singlet bound states that have already hadronized. They have been termed hyper-confining modes by Rothstein [86]. The soft-collinear messenger mode considered in Refs. [75, 77, 80] are in the hyperconfining category.

${ }^{20}$ Note that in general matching computations between QCD and $\mathrm{SCET}_{\mathrm{II}}$ can be a bit tricky because one must be sure that ones choice of IR regulator is treating the Hilbert space of full QCD in exactly the same way as the Hilbert space of $\mathrm{SCET}_{\mathrm{II}}$. An example of this type that we encountered in $\mathrm{SCET}_{\mathrm{I}}$ was our discussion of the field redefined current $J^{\prime(0)}$ in $b \rightarrow s \gamma$ in sections $\mathrm{VIA}$ and VIB. For SCET $\mathrm{II}$ further discussion of this point is given in Appendix B. 


\section{a) full theory graphs}
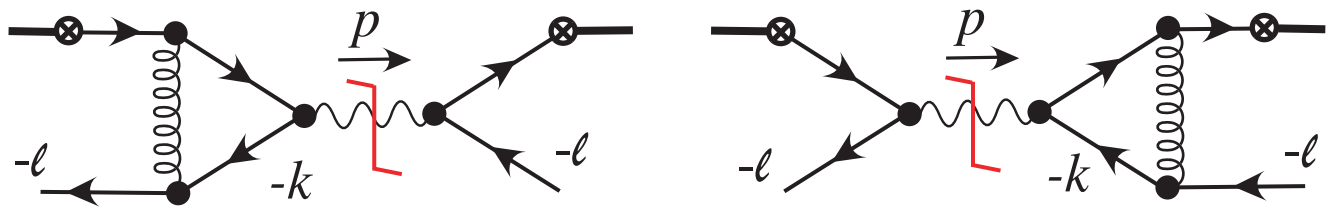

b) SCET $_{\text {II }}$ soft graphs
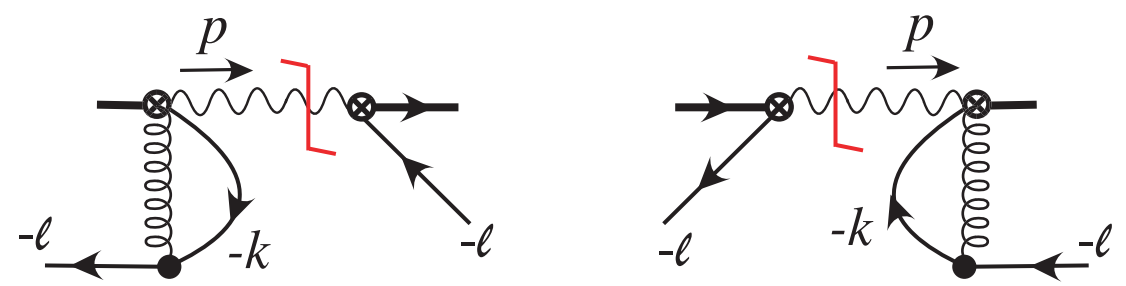

\section{c) SCET $_{\text {II }}$ collinear graphs}
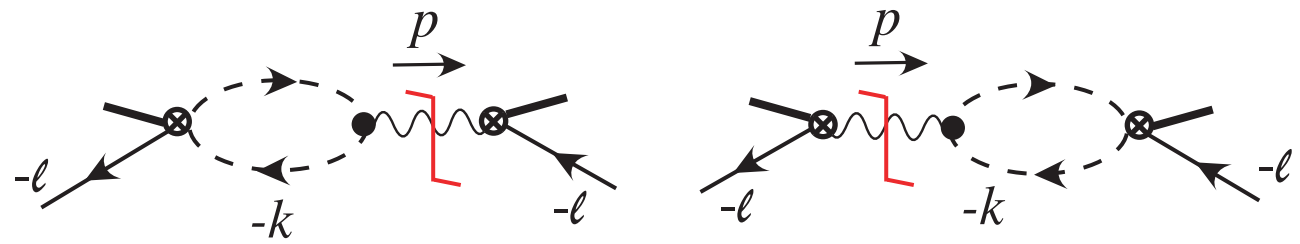

FIG. 14: One loop cut-graphs for the $\gamma$-fragmentation contribution to $B \rightarrow \gamma \ell \bar{\nu}$ in the full and effective theories. The $\otimes$ 's denote an insertion of the weak current and the leptons are not shown. The graphs in a) are in full QCD, while the graphs in b) and c) are in SCET II . For b) we have soft propagators, while for $\mathrm{c}$ ) the propagators are collinear (dashed).

with standard kinetic terms for $\phi_{b}$ with mass $m_{b}$, and for the massless charged and neutral scalars. Here the coupling $G$ tracks the current, and $g$ and $e$ are coupling constants of mass dimension one. In $\mathrm{SCET}_{\mathrm{II}}$ the leading order Lagrangian is split into soft and collinear fields,

$$
\begin{aligned}
\mathcal{L}_{\mathrm{II}}^{(0)} & =2 m_{b} \phi_{v}^{b \dagger} i v \cdot \partial \phi_{v}^{b}+\phi_{s}^{\dagger}(i \partial)^{2} \phi_{s}+\frac{1}{2} \phi_{s}^{g}(i \partial)^{2} \phi_{s}^{g}+g\left[\phi_{s, \ell-k}^{g} \phi_{s, \ell}^{\dagger} \phi_{s, k}\right] \\
& +\phi_{n}^{\dagger}(i \partial)^{2} \phi_{n}+\frac{1}{2} \phi_{n}^{\gamma}(i \partial)^{2} \phi_{n}^{\gamma}+\frac{1}{2} \phi_{n}^{g}(i \partial)^{2} \phi_{n}^{g}+g\left[\phi_{n, p-q}^{g} \phi_{n, p}^{\dagger} \phi_{n, q}\right]+e\left[\phi_{n, p-q}^{\gamma} \phi_{n, p}^{\dagger} \phi_{n, q}\right],
\end{aligned}
$$

where $\phi_{v}^{b}$ is a scalar HQET field [58], the $\phi_{s}$ and $\phi_{n}$ are soft and collinear massless "quarks", and $\phi_{s}^{g}, \phi_{n}^{g}$, and $\phi_{n}^{\gamma}$ are scalars for the "gauge" fields. The collinear fields have label momenta $p^{-} \sim \eta^{0}$ and residual momenta $p_{r}^{\perp} \sim \eta$ and $p_{r}^{+} \sim \eta^{2}$, and the soft fields have label momenta $k^{+} \sim \eta$ and residual momenta $k_{r}^{\perp} \sim \eta$ and $k_{r}^{-} \sim \eta$. Writing out the label and residual terms explicitly in the kinetic terms we would have $\left(i \partial^{\mu}\right) \phi_{s} \rightarrow\left(\bar{n}^{\mu} \mathcal{P} / 2+i \partial_{r}^{\mu}\right) \phi_{s, \ell}$, $\left(i \partial^{\mu}\right) \phi_{n} \rightarrow\left(n^{\mu} \overline{\mathcal{P}} / 2+i \partial_{r}^{\mu}\right) \phi_{n, p}$ etc., with the standard treatment of leading and subleading terms. Demanding $\mathcal{L}^{(0)} \sim \eta^{0}$ the power counting in $\eta$ is

$$
\phi_{v}^{b} \sim \eta^{3 / 2}, \quad \phi_{s} \sim \phi_{n} \sim \eta, \quad \phi_{n}^{\gamma} \sim \eta, \quad \phi_{s}^{g} \sim \phi_{n}^{g} \sim \eta .
$$


The leading order currents we will need are

$$
\begin{aligned}
O_{\mathrm{II}}^{(0 a)} & =\sum_{p^{-}, \ell^{+} \neq 0} \frac{J^{(0 a)}}{\left(\bar{n} \cdot p n \cdot \ell-i 0^{+}\right)}\left[\phi_{s,-\ell}^{\dagger} \phi_{v}^{b} \phi_{n, p}^{\gamma}\right], \\
O_{\mathrm{II}}^{(0 b)} & =\sum_{p^{-}, \ell^{+}, k^{+} \neq 0} \frac{J^{(0 b)}}{\left(\bar{n} \cdot p n \cdot \ell-i 0^{+}\right)\left(\bar{n} \cdot p n \cdot k-i 0^{+}\right)}\left[\phi_{s,-k}^{\dagger} \phi_{v}^{b} \phi_{n, p}^{\gamma} \phi_{s, \ell-k}^{g}\right], \\
O_{\mathrm{II}}^{(0 c)} & =\sum_{p^{-}, \ell^{+}, q^{-} \neq 0} \frac{J^{(0 c)}}{\left(\bar{n} \cdot p n \cdot \ell-i 0^{+}\right)\left(\bar{n} \cdot q n \cdot \ell-i 0^{+}\right)}\left[\phi_{s,-\ell}^{\dagger} \phi_{v}^{b} \phi_{n, p-q}^{\dagger} \phi_{n,-q}\right],
\end{aligned}
$$

where $\bar{n} p \sim \eta^{0}$ while $n \ell \sim n \cdot k \sim \eta$. Note that the operators $O_{\mathrm{II}}^{(0 b)}$ and $O_{\mathrm{II}}^{(0 c)}$ include restrictions on the label sums with $n \cdot \ell \neq 0$ and $\bar{n} \cdot p \neq 0$ respectively. In these bins the $1 /(\bar{n} \cdot p n \cdot \ell)$ factor would be a collinear or soft propagator in $\mathrm{SCET}_{\mathrm{II}}$, and these bins are taken into account by time-ordered products with subleading $\mathrm{SCET}_{\mathrm{II}}$ Lagrangians as discussed in Ref. [27.

Note that in scalar $\mathrm{SCET}_{\mathrm{II}}$, we can construct leading order operators with additional scalar fields since the extra powers of $\eta$ are compensated by $1 /(\bar{n} \cdot p n \cdot \ell)$ factors and the mass dimension is compensated by the dimension of the couplings. In our perturbative example only the currents shown are needed (plus counterterm operators). A non-perturbative treatment would require additional terms. In the scalar theory, matching tree level graphs gives $J^{(0 a)}=e G, J^{(0 b)}=e g G$, and $J^{(0 c)}=g^{2} G$. In dimensional regularization, the currents are modified in a manner described in section VIIB.

In the gauge theory with fermions, the full set of operators for $B \rightarrow \gamma e \bar{\nu}$ would be determined by gauge invariance and leading order matching. The current analogous to $O_{\mathrm{II}}^{(0 a)}$ is LO, while the currents analogous to $O_{\mathrm{II}}^{(0 b, 0 c)}$ are suppressed by one power of $\eta$. The non-perturbative treatment is simpler in gauge theories since it is constrained by more

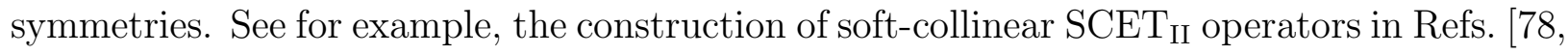
87, 88.

A goal of this section is to demonstrate that although there is not a simple factorization for the scalar $B \rightarrow \gamma e \bar{\nu}$ process of the form $J(\mu) \otimes \phi_{n}(\mu) \otimes \phi_{s}(\mu)$, there appears to be a more involved factorization which contains terms of the form

$$
\int d k^{+} d p^{-} J\left(k^{+}, p^{-}, \mu_{+}, \mu_{-}\right) \phi_{n}\left(p^{-}, \mu_{-}, \mu^{2}\right) \phi_{s}\left(k^{+}, \mu_{+}, \mu^{2}\right) .
$$

Here $J$ is a perturbative jet function, and the $\phi$ 's are non-perturbative, with $\phi_{n}$ given by a matrix element of collinear fields and $\phi_{s}$ given by a matrix element of soft fields. In Eq. (92) we have two additional scale parameters $\mu_{+}$and $\mu_{-}$in $\mathrm{SCET}_{\mathrm{II}}$ (or two factorization scales in a more traditional language in full QCD). We will demonstrate that these scales are connected in a specific way to the standard renormalization scale

$$
\mu_{-} \mu_{+}=\mu^{2}
$$

Although our conjecture about the existence of a factorization formula valid to all orders in $\alpha_{s}$ differs from Refs. [77, 78, 80], the structure of the result is also different from standard 
factorization formulas in the literature. Because we do not have the identity $\mu_{+}=\mu_{-}=\mu$, there is not a simple factorization for the amplitude for this process, in agreement with the conclusions in Refs. [77, 78, 80]. The perturbative formula that we find for the observable process, Eq. (86), has all the desired properties of a factorization formula, including correctly reproducing IR divergences (from the zero-bin subtractions), finite convolution integrals (from the zero-bin and renormalization), and distinct matrix elements for the soft and collinear objects.

In the context of the analytic IR regulator used in [78], it was pointed out that there was an interesting cancellation between IR divergences in soft and collinear diagrams. Our observation is that this cancellation has to do with avoiding double counting just like the zero-bin subtractions, rather than having to do with reproducing IR divergences in QCD. For physical observables, like the forward scattering graphs, the method for avoiding the double counting is computable, and can be handled in perturbation theory. It results in regularization parameters $\mu_{+}$and $\mu_{-}$which encode the coupling between soft and collinear modes with a simple correlation. Our results turn situations which were previously plagued by the unphysical convolution endpoint singularities, into manageable finite amplitudes, which one can then try to arrange into a predictive factorization formula. Since the divergent effects are computable they do not spoil many of the nice features obtained in simpler QCD factorization formulas.

\section{Soft-Collinear Division with a Hard Cutoff Regulator}

We begin by considering a hard cutoff, $a$, between the soft and collinear modes, as indicated in Fig. 13. For a loop momentum $k^{\mu}$ we define $\zeta_{k}=\bar{n} \cdot k / n \cdot k=k^{-} / k^{+}$as discussed near Eq. (15). Only the magnitude of the rapidity variable, $\left|\zeta_{k}\right|$, is relevant for distinguishing the soft and collinear modes. Switching variables from $\left\{k^{+}, k^{-}\right\}$to $\left\{k^{+}, \zeta_{k}\right\}$ gives $d k^{-}=\left|k^{+}\right| d \zeta_{k}$ when we integrate over $-\infty<k^{+}<\infty$ and $-\infty<\zeta_{k}<\infty$, so the loop integral is no longer analytic in $k^{+}$but remains analytic in $\zeta_{k}$, and likewise if we switch to $\left\{k^{-}, \zeta_{k}\right\}$. When imposing hard cutoffs we need to avoid the physical poles, which can be accomplished using cutoffs in Euclidean space after Wick rotation. For variables $\left\{k^{+}, \zeta_{k}\right\}$ the Wick rotation $k^{-} \rightarrow i k^{-}$is equivalent to $\zeta_{k}=i \zeta_{k}^{\prime}$, while for $\left\{k^{-}, \zeta_{k}\right\}$ the Wick rotation

$k^{+} \rightarrow i k^{+}$gives $\zeta_{k}=-i \zeta_{k}^{\prime}$. In our examples Wick rotation about the origin suffices, and the poles in complex $\zeta_{k}^{\prime}$ occur along the imaginary axis in the first and third quadrants. We take cutoffs

$$
\begin{aligned}
\text { soft: } & -a^{2} \leq \zeta_{k}^{\prime} \leq a^{2}, \\
\text { collinear: } & -a^{2} \geq \zeta_{k}^{\prime} \quad \text { or } \quad \zeta_{k}^{\prime} \geq a^{2} .
\end{aligned}
$$

As mentioned above we only require $n$-collinear and soft fields in $\mathrm{SCET}_{\mathrm{II}}$ for the example in this section and so are free to include the entire $\zeta_{k}^{\prime} \lesssim 1$ region in the soft modes. (For more 


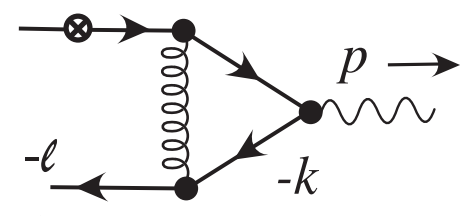

a)

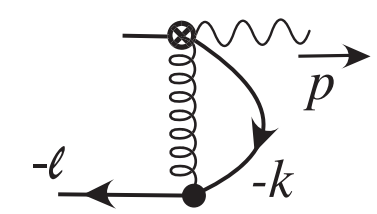

b)

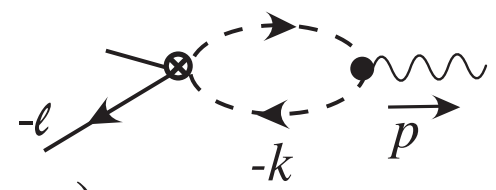

c)

FIG. 15: Graphs with scalar propagators as a toy model for the $\gamma$-fragmentation contribution to $B \rightarrow \gamma \ell \bar{\nu}$ with fermions. The $\otimes$ denotes the weak current and the leptons are not shown. Graph a) is in full QCD, graph b) has an insertion of $O_{\mathrm{II}}^{(0 b)}$ and a loop with soft fields in $\mathrm{SCET}_{\mathrm{II}}$, and graph c) has an insertion of $O_{\mathrm{II}}^{(0 c)}$ and a collinear loop (with dashed propagators).

complicated problems the region $\zeta_{k}^{\prime} \sim \eta^{2}$ would need to be disentangled for the $\bar{n}$-collinear modes.) We can take $a^{2} \sim \eta$, and note that under an RPI-III transformation on $n$ and $\bar{n}$ [79 (a longitudinal boost) that $a^{2}$ behaves like a $\left(p^{-}\right)^{2}$ momentum just like $\zeta_{k}$ does. Also note that the power counting scaling which fixes the soft and collinear components only depends on $\left|\zeta_{k}\right|$ or $\left|\zeta_{k}^{\prime}\right|$ and so does not care about the Wick rotation.

For simplicity we consider the same diagram as discussed in Ref. [78] which is shown in Fig. 15a. Unlike Ref. [78, we do not analyze this graph with the method of regions. Instead we consider the diagram in full scalar field theory (Fig. 15a) and the corresponding diagrams in scalar $\mathrm{SCET}_{\mathrm{II}}$ (Fig. 15b,c). The difference of the two results gives a matching contribution, and allows us to check that the full theory IR divergences are correctly reproduced with zero-bin subtractions implemented in $\mathrm{SCET}_{\mathrm{II}}$. It also allows us to discuss the factorization formula in Eq. (86).

For simplicity we will leave off the prefactor $i e g^{2} G /\left(p^{-} \ell^{+}\right)$in quoting results for graphs in this subsection. For the full theory diagram, we have the integral

$$
I_{\text {full }}^{\text {scalar }}=\int \frac{d^{D} k}{(2 \pi)^{D}} \frac{1}{\left[(k-\ell)^{2}+i 0^{+}\right]\left[k^{2}+i 0^{+}\right]\left[(k-p)^{2}+i 0^{+}\right]} .
$$

Evaluating this onshell with $p^{\mu}=p^{-} n^{\mu} / 2, \ell^{\mu}=\ell^{+} \bar{n}^{\mu} / 2$, so that $p^{2}=\ell^{2}=0$, we have

$$
I_{\text {full }}^{\text {scalar }}=\frac{-i}{16 \pi^{2}\left(p^{-} \ell^{+}\right)}\left[\frac{1}{\epsilon_{\mathrm{IR}}^{2}}-\frac{1}{\epsilon_{\mathrm{IR}}} \ln \left(\frac{p^{-} \ell^{+}}{\mu^{2}}\right)+\frac{1}{2} \ln ^{2}\left(\frac{p^{-} \ell^{+}}{\mu^{2}}\right)-\frac{\pi^{2}}{12}\right] .
$$

Here the IR divergences are regulated by dimensional regularization. For the soft and collinear graphs in Fig. 15b,c we find

$$
\begin{aligned}
I_{\mathrm{soft}}^{\mathrm{scalar}} & =\sum_{k^{+} \neq 0} \int \frac{d^{D} k_{r}}{(2 \pi)^{D}} \frac{1}{\left[k^{2}-n \cdot \ell \bar{n} \cdot k+i 0^{+}\right]\left[k^{2}+i 0^{+}\right]\left[-\bar{n} \cdot p n \cdot k+i 0^{+}\right]}, \\
I_{\mathrm{nc}}^{\mathrm{scalar}} & =\sum_{k^{-} \neq 0} \int \frac{d^{D} k_{r}^{\prime}}{(2 \pi)^{D}} \frac{1}{\left[-n \cdot \ell \bar{n} \cdot k+i 0^{+}\right]\left[k^{2}+i 0^{+}\right]\left[k^{2}-\bar{n} \cdot p n \cdot k+i 0^{+}\right]},
\end{aligned}
$$

where in both cases the first two terms are the displayed propagators, and the last factor comes from the non-local vertex which emits the scalar soft or collinear fields in $\mathrm{SCET}_{\mathrm{II}}$. 
To compute the EFT graphs we implement the hard cutoff in Eq. (94) to regulate UV effects in the effective theory diagrams. With this regulator the zero-bin subtractions are automatically zero since they are outside the region of integration. The hard cutoffs are theta functions in the integrand so they give identically zero for the integrand evaluated in the subtraction regions. Therefore with this regulator the full integrals are given by the naive replacement in Eq. (16). We discuss in detail the calculation of the SCET II diagrams in Appendix A. For the soft graph the result is

$$
\begin{aligned}
I_{\mathrm{soft}}^{\mathrm{scalar}}=\tilde{I}_{\mathrm{soft}}^{\mathrm{scalar}} & =\frac{-i}{16 \pi^{2}\left(p^{-} \ell^{+}\right)}\left[\frac{1}{2 \epsilon_{\mathrm{IR}}^{2}}-\frac{1}{\epsilon_{\mathrm{IR}}} \ln \left(\frac{\ell^{+} a}{\mu}\right)+\ln ^{2}\left(\frac{\ell^{+} a}{\mu}\right)-\frac{\pi^{2}}{16}\right] \\
& =\frac{-i}{16 \pi^{2}\left(p^{-} \ell^{+}\right)}\left[\frac{1}{2 \epsilon_{\mathrm{IR}}^{2}}-\frac{1}{\epsilon_{\mathrm{IR}}} \ln \left(\frac{\ell^{+}}{\mu_{+}}\right)+\ln ^{2}\left(\frac{\ell^{+}}{\mu_{+}}\right)-\frac{\pi^{2}}{16}\right],
\end{aligned}
$$

where we defined $\mu_{+}=\mu / a$. Note that since $a$ boosts like a minus-momentum, $\mu_{+}$behaves like a plus-momentum, and the result in Eq. (98) is RPI-III invariant. For the collinear graph the result is

$$
\begin{aligned}
I_{\mathrm{cn}}^{\text {scalar }}=\tilde{I}_{\mathrm{cn}}^{\text {scalar }} & =\frac{-i}{16 \pi^{2}\left(p^{-} \ell^{+}\right)}\left[\frac{1}{2 \epsilon_{\mathrm{IR}}^{2}}-\frac{1}{\epsilon_{\mathrm{IR}}} \ln \left(\frac{p^{-}}{a \mu}\right)+\ln ^{2}\left(\frac{p^{-}}{a \mu}\right)-\frac{\pi^{2}}{16}\right], \\
& =\frac{-i}{16 \pi^{2}\left(p^{-} \ell^{+}\right)}\left[\frac{1}{2 \epsilon_{\mathrm{IR}}^{2}}-\frac{1}{\epsilon_{\mathrm{IR}}} \ln \left(\frac{p^{-}}{\mu_{-}}\right)+\ln ^{2}\left(\frac{p^{-}}{\mu_{-}}\right)-\frac{\pi^{2}}{16}\right],
\end{aligned}
$$

where we defined $\mu_{-}=a \mu$. Here $\mu_{-}$behaves like a minus-momentum and the result in Eq. (99) is also RPI-III invariant. The soft and collinear regularization parameters $\mu_{ \pm}$ defined in the computation of Eqs. (98) and (99) obey the anticipated relation,

$$
\mu_{+} \mu_{-}=\mu^{2}
$$

where the $a$ dependence cancels out in this product. Moreover, with $a^{2} \sim \eta$ we find that with $\mu^{2}$ at the matching scale, $\mu^{2} \sim Q \Lambda_{\mathrm{QCD}}$, one can still take $\mu_{+} \gtrsim \Lambda_{\mathrm{QCD}}$ and $\mu_{-} \sim Q$. Thus we can simultaneously minimize the logarithms in the $\mathrm{SCET}_{\mathrm{II}}$ matrix elements, which in our perturbative computation are represented by $I_{\text {soft }}^{\text {scalar }}$ and $I_{\mathrm{cn}}^{\text {scalar }}$.

Adding the two SCET $\mathrm{II}$ graphs, $I_{\mathrm{s}+\mathrm{cn}}^{\text {scalar }}=I_{\mathrm{s}}^{\text {scalar }}+I_{\mathrm{cn}}^{\text {scalar }}$, we find

$$
\begin{aligned}
I_{\mathrm{s}+\mathrm{cn}}^{\mathrm{scalar}} & =\frac{-i}{16 \pi^{2}\left(p^{-} \ell^{+}\right)}\left[\frac{1}{\epsilon_{\mathrm{IR}}^{2}}-\frac{1}{\epsilon_{\mathrm{IR}}} \ln \left(\frac{p^{-} \ell^{+}}{\mu_{-} \mu_{+}}\right)+\ln ^{2}\left(\frac{p^{-}}{\mu_{-}}\right)+\ln ^{2}\left(\frac{\ell^{+}}{\mu_{+}}\right)-\frac{\pi^{2}}{8}\right] \\
& =\frac{-i}{16 \pi^{2}\left(p^{-} \ell^{+}\right)}\left[\frac{1}{\epsilon_{\mathrm{IR}}^{2}}-\frac{1}{\epsilon_{\mathrm{IR}}} \ln \left(\frac{p^{-} \ell^{+}}{\mu^{2}}\right)+\ln ^{2}\left(\frac{p^{-}}{\mu_{-}}\right)+\ln ^{2}\left(\frac{\ell^{+}}{\mu_{+}}\right)-\frac{\pi^{2}}{8}\right] .
\end{aligned}
$$

We see that with the relation $\mu^{2}=\mu_{+} \mu_{-}$, the $1 / \epsilon_{\mathrm{IR}}$ poles agree exactly with the full theory expression in Eq. (96) as required. To match the full and effective calculations we set $\mu^{2}=\mu_{+} \mu_{-}$and subtract to find

$$
\begin{aligned}
I_{\text {matching }}^{\text {scalar }} & =\frac{-i}{16 \pi^{2}\left(p^{-} \ell^{+}\right)}\left[+\frac{1}{2} \ln ^{2}\left(\frac{p^{-} \ell^{+}}{\mu_{-} \mu_{+}}\right)-\ln ^{2}\left(\frac{p^{-}}{\mu_{-}}\right)-\ln ^{2}\left(\frac{\ell^{+}}{\mu_{+}}\right)+\frac{\pi^{2}}{24}\right] \\
& =\frac{-i}{16 \pi^{2}\left(p^{-} \ell^{+}\right)}\left[-\frac{1}{2} \ln ^{2}\left(\frac{p^{-} \mu_{+}}{\mu_{-} \ell^{+}}\right)+\frac{\pi^{2}}{24}\right] .
\end{aligned}
$$


Here the $\ln ^{2}\left(p^{-} \mu_{+} / \ell^{+} \mu_{-}\right)$contributes $p^{-}$and $\ell^{+}$dependence to the jet function $J\left(\ell^{+}, p^{-}, \mu_{+}, \mu_{-}\right)$at one loop. To minimize the large logarithms in the matching calculation in Eq. (102) we take $\mu^{2}=\mu_{-} \mu_{+}$to be of order the hard-collinear scale, and take $\mu_{+} / \mu_{-} \sim \eta$. Since the matching result in Eq. (102) depends on $\mu_{+} / \mu_{-}$it depends on $a$, which is not surprising. Here $a$ ensures there is no double counting between the soft and collinear modes in the IR, but $a$ also changes the behavior of the collinear and soft modes in the ultraviolet. This change is compensated by the perturbative Wilson coefficient, and in perturbation theory the sum of these contributions reproduce the full theory result.

The result in Eq. (102) is shown for illustration only, since a complete matching calculation for scalar $B \rightarrow \gamma \ell \bar{\nu}$ requires a computation of all diagrams, not just the one diagram that we considered. For example, one should also compute graphs with the scalar gluon attached to the $b$-quark line, and wavefunction renormalization type diagrams in both the full and effective theories.

\section{B. Dimensional Regularization Division in $\mathrm{SCET}_{\mathrm{II}}$ : General Discussion}

In this section, we discuss the use of dimensional regularization for the UV divergences and the separation of soft and collinear modes. This regulator makes higher order computations more feasible and preserves gauge symmetry. We also expect that it will make it easier to compute anomalous dimensions and sum logarithms using renormalization group techniques, although we do not address these features here. Finally, it is useful to consider dimensional regularization in order to compare how the separation of modes in rapidity space appears with a different regulator.

Since the standard application of dimensional regularization is boost invariant, it does not provide the ability to distinguish modes in rapidity space. This also means that in general, divergences in the rapidity will not be regulated by standard dimensional regularization. For an insertion of a mixed soft-collinear operator, we can regulate the rapidity space in dimensional regularization by scaling out factors of the label operators from the Wilson coefficients. To implement Fig. 13 in dimensional regularization, the correct form of the operators are

$$
\begin{aligned}
& J\left(p_{j}^{-}, k_{j}^{+}\right)\left[\left(\bar{q}_{s} S\right)_{k_{1}^{+}} \Gamma_{s}\left(S^{\dagger} q_{s}\right)_{k_{2}^{+}}\right]\left[\left(\bar{\xi}_{n} W\right)_{p_{1}^{-}} \Gamma_{n}\left(W^{\dagger} \xi_{n}\right)_{p_{2}^{-}}\right] \\
& \stackrel{\text { dim.reg. }}{\longrightarrow} J\left(p_{j}^{-}, k_{j}^{+}, \mu_{ \pm}\right) \mu^{2 \epsilon}\left[\left(\bar{q}_{s} S\right)_{k_{1}^{+}} \frac{\left|\mathcal{P}^{\dagger}\right| \epsilon}{\mu_{+}^{\epsilon}} \Gamma_{s} \frac{|\mathcal{P}|^{\epsilon}}{\mu_{+}^{\epsilon}}\left(S^{\dagger} q_{s}\right)_{k_{2}^{+}}\right]\left[\left(\bar{\xi}_{n} W\right)_{p_{1}^{-}} \frac{\left|\overline{\mathcal{P}}^{\dagger}\right| \epsilon}{\mu_{-}^{\epsilon}} \Gamma_{n} \frac{|\overline{\mathcal{P}}|^{\epsilon}}{\mu_{-}^{\epsilon}}\left(W^{\dagger} \xi_{n}\right)_{p_{2}^{-}}\right] \\
& \quad=J\left(p_{j}^{-}, k_{j}^{+}, \mu_{ \pm}, \mu^{2}\right) \mu^{2 \epsilon}\left[\frac{\left|k_{1}^{+} k_{2}^{+}\right|^{\epsilon}}{\mu_{+}^{2 \epsilon}}\left(\bar{q}_{s} S\right)_{k_{1}^{+}} \Gamma_{s}\left(S^{\dagger} q_{s}\right)_{k_{2}^{+}}\right]\left[\frac{\left|p_{1}^{-} p_{2}^{-}\right|^{\epsilon}}{\mu_{-}^{2 \epsilon}}\left(\bar{\xi}_{n} W\right)_{p_{1}^{-}} \Gamma_{n}\left(W^{\dagger} \xi_{n}\right)_{p_{2}^{-}}\right] .
\end{aligned}
$$

Here the label operator $\mathcal{P}$ gives the plus momentum from soft fields, and the label operator $\overline{\mathcal{P}}$

gives the minus momentum from collinear fields. The momenta subscripts occur for products of quark fields and Wilson lines, $\left(S^{\dagger} q_{s}\right)_{k^{+}}=\delta\left(k^{+}-\mathcal{P}\right)\left(S^{\dagger} q_{s}\right),\left(W^{\dagger} \xi_{n}\right)_{p^{-}}=\delta\left(p^{-}-\overline{\mathcal{P}}\right)\left(W^{\dagger} \xi_{n}\right)$, 
which ensures that the momenta are gauge invariant and that the gauge symmetry is not spoiled by the factors of $|\overline{\mathcal{P}}|^{\epsilon},|\mathcal{P}|^{\epsilon}$, etc. The absolute values ensure that we raise a positive physical momentum to the $\epsilon$ power, and thus do not modify the cut structure of matrix elements. ${ }^{21} J$ is the Wilson coefficient jet function.

This rescaling will allow us to properly distinguish the soft and collinear modes in dimensional regularization without imposing a hard cutoff to implement the division in Fig. 13. This modification of the current is not done to solve a problem in the IR - it is the zero-bin subtractions for the soft and collinear fields which will ensure that there is no IR double counting. The zero-bin subtraction terms are integrated over all space, which introduces new UV divergences in rapidity space, and in Eq. (103) the factors of $|\overline{\mathcal{P}}|^{\epsilon}$ etc. are necessary to regulate these UV divergences. If one thinks of splitting the full loop integral $I$ into a naive part $\tilde{I}$ and a subtraction part $I_{0}$, then $\tilde{I}$ has an IR rapidity divergence, while $I_{0}$ has both UV and IR divergences. The IR divergences cancel in $I=\tilde{I}-I_{0}$, so the rapidity divergence in $I$ is pure $\mathrm{UV}$. We will see that these remaining UV divergences can be removed by counterterms.

Before giving the rules for constructing Eq. (103), let us consider how it should be used. When we do a collinear loop involving an insertion of this operator we expand in $\left(p^{-} / \mu_{-}\right)^{\epsilon}$, but DO NOT expand the $\left(k^{+} / \mu_{+}\right)^{\epsilon}$ factors, and we do the opposite for a soft loop. This dimensional regularization rule is forced on us in any field theory with a multipole expansion, and $\mathrm{SCET}_{\mathrm{II}}$ has a multipole expansion between components of the soft and collinear momenta. The rule was discussed in Ref. [53] for NRQCD in examples involving mixed usoft-soft loops. In general one does not expand matrix elements of the soft fields in $D$ dimensions when doing the collinear loops and one does not expand matrix elements of the collinear fields when doing soft loops. The factors of $\left(p^{-} / \mu_{-}\right)^{\epsilon}$ and $\left(k^{+} / \mu_{+}\right)^{\epsilon}$ should be thought of as being associated with the renormalized coupling function $J$, just like a factor of $\mu^{\epsilon}$ is associated to the strong coupling $g(\mu)$. For purely collinear or purely soft operators we apply dimensional regularization in the usual manner. The only place that $\mu_{ \pm}$ appear is in the insertion of a mixed soft-collinear operator. All purely soft operators and purely collinear operators only have $\mu^{\epsilon}$ factors, and so all couplings are $\alpha_{s}(\mu)$. The couplings do not dependent explicitly on $\mu_{ \pm}$. In multiloop diagrams one can carry out the standard renormalization procedure first, and leave to the end the rapidity renormalization for the final loop involving the soft-collinear vertex.

${ }^{21}$ Recall that the labels are positive for particles, and negative for antiparticles [3]. Combining both particles and antiparticles into a single field distinguished by the sign of the label simplifies the formulation of the effective theory. One could instead have used separate fields for the particles and antiparticles, in which case the antiparticle field could also be chosen to have a positive label. The absolute values in $|\mathcal{P}|^{\epsilon}$ mean that we are using the momentum of the particle, which is unambiguous, rather than the label on the field, which is convention dependent. Due to the zero-bin conditions $p_{i}^{-} \neq 0$ and $k_{i}^{+} \neq 0$, there is no problem at the origin. 
Lets consider how we determined the powers of $\mu_{+}, \mu_{-}$and $\mu$ for Eq. (103). In dimensional regularization, factors of $\mu^{\epsilon}$ appear from ensuring that coupling constants in the action are dimensionless. Demanding that this is the case for $J$ gives the $\mu^{2 \epsilon}$ factor. To determine the factors of $\mu_{ \pm}$we must examine the scaling of fields in the operator along the solid red curve in Fig. 13. An RPI-III transformation scales all modes by a common amount and separate invariance under this transformation demands we introduce a $1 / \mu_{+}$to compensate each $\mathcal{P}$, and a $1 / \mu_{-}$for each $\overline{\mathcal{P}}$. Charge conjugation requires the same power for quarks and antiquarks, and the boost-inversion symmetry [75, 77] requires the same power be used for the soft and collinear fields. We can also demand rapidity invariance under small individual scalings of the soft and collinear sectors. This will determine the power of the label parameters, $|\overline{\mathcal{P}}|^{\epsilon}$ and $|\mathcal{P}|^{\epsilon}$. The power is related to the space-time dimension because in doing this rapidity scaling we demand that the invariant mass $p^{2}$ remains homogeneous. As an example consider a scaling by $\beta>0: \chi_{n, p^{-}}(0) \rightarrow \chi_{n, \beta p^{-}}(0)=\beta^{-\epsilon} \chi_{n, p^{-}}(0)$, where here $x=0$ as in the soft-collinear operator. To derive the $\beta^{-\epsilon}$ factor write the quark field $\chi_{n, \beta p^{-}}(0)=\delta\left(\beta p^{-}-\overline{\mathcal{P}}\right) \chi_{n}(0)=\int d^{d} k \delta\left(\beta p^{-}-k^{-}\right) \delta\left(k^{2}\right) \theta\left(k^{-}\right) a_{k}$, and then shift $k^{-} \rightarrow \beta k^{-}$ and $k_{\perp}^{2} \rightarrow \beta k_{\perp}^{2}$ (to keep $k^{2}$ homogeneous). This $\beta^{-\epsilon}$ factor from the transformation of $\chi_{n, p^{-}}$ is exactly canceled by the $|\overline{\mathcal{P}}|^{\epsilon} \rightarrow|\overline{\mathcal{P}}|^{\epsilon} \beta^{\epsilon}$ factor acting on this field. The symmetry of the problem dictates that we need one such factor for each collinear field. Repeating these arguments with a scaling parameter $\beta^{\prime}$ in the soft-sector determines the $|\mathcal{P}|^{\epsilon}$ terms.

These arguments determine the proper operator for the dimensional regularization computations in $\mathrm{SCET}_{\mathrm{II}}$, with an example shown in Eq. (103). The Wilson coefficient $J$ has non-trivial $\mu_{-}$and $\mu_{+}$dependence which cancels the dependence on these parameters in the matrix element of the effective theory operator order by order in $\alpha_{s}(\mu)$. Thus we see that factorizing the soft and collinear modes in $\mathrm{SCET}_{\mathrm{II}}$ also requires introducing $\mu_{+}$and $\mu_{-}$, just like with our cutoff regulator.

It is interesting to compare the regulator introduced in Eq. (103) with the use of analytic regulators used for collinear computations in Refs. [78, 89, 90]. Much like an analytic regulator, the result in Eq. (103) modifies the power of a momentum dependent factor in the integrand. It is used to regulate divergences that are not handled by dimensional regularization, which is also the motivation for introducing an analytic regulator. However, unlike the use of analytic regulators, Eq. (103) is gauge invariant, defines the modification at the operator level, and does not modify the power of the propagators in the EFT. Furthermore, as already emphasized, with our zero-bin setup this regulator is needed for divergences in the UV, rather than the IR. Since these divergences arise due to the separation of momentum fractions in hard scattering kernels and collinear operators, we anticipate that the addition of a power of the momentum fraction for each labeled field will regulate UV rapidity divergences in a general situation. 


\section{Dimensional Regularization for the one-loop example}

We now repeat the computation in the last section with dimensional regularization for the UV. The loop integral in Eq. (95) has IR divergences for $k \rightarrow 0, k \rightarrow \ell^{+}$, and $k \rightarrow p^{-}$. We take one of the propagator lines to have an infinitesimal mass $m^{2}$ to regulate these IR divergences. For simplicity we will leave off the prefactor $i e g^{2} G /\left(p^{-} \ell^{+}\right)$in quoting results. For the full theory diagram we have

$$
\begin{aligned}
I_{\text {full }}^{\text {scalar }} & =\int \frac{d^{D} k}{(2 \pi)^{D}} \frac{1}{\left[(k-\ell)^{2}+i 0^{+}\right]\left[k^{2}-m^{2}+i 0^{+}\right]\left[(k-p)^{2}+i 0^{+}\right]} \\
& =\frac{-i}{16 \pi^{2}\left(p^{-} \ell^{+}\right)}\left[\frac{1}{2} \ln ^{2}\left(\frac{m^{2}}{p^{-} \ell^{+}}\right)+\frac{\pi^{2}}{3}\right] .
\end{aligned}
$$

Here $m^{2}$ regulates the IR divergences in a manner similar to the solid red curve in Fig. 13. Other choices of IR regulator can be made, and in Appendix B we repeat the computations done in this section with i) factors $m_{2}^{2}, m_{1}^{2}$, and $m_{3}^{2}$ in the three propagators in Eq. (104), and ii) taking $p^{2} \neq 0$ and $\ell^{2} \neq 0$ in Eq. (104). The choice $m_{1}=0$ is also discussed in Appendix B, but makes the matching more complicated.

The LO mixed soft-collinear $\mathrm{SCET}_{\mathrm{II}}$ currents in dimensional regularization include the UV rapidity regulation factors, and are

$$
\begin{aligned}
O_{\mathrm{II}}^{(0 a)} & =\frac{J^{(0 a)}}{\left(\bar{n} \cdot p n \cdot \ell-i 0^{+}\right)}\left[\phi_{s,-\ell}^{\dagger} \phi_{v}^{b} \frac{\left|\ell^{+}\right|^{\epsilon}}{\mu_{+}^{\epsilon}}\right]\left[\phi_{n, p}^{\gamma} \frac{\left|p^{-}\right|^{\epsilon}}{\mu_{-}^{\epsilon}}\right], \\
O_{\mathrm{II}}^{(0 b)} & =\frac{J^{(0 b)}}{\left(\bar{n} \cdot p n \cdot \ell-i 0^{+}\right)\left(\bar{n} \cdot p n \cdot k-i 0^{+}\right)}\left[\phi_{s,-k}^{\dagger} \phi_{v}^{b} \phi_{s, \ell-k}^{g} \frac{\left.\left|\ell^{+}-k^{+}\right|\right|^{\epsilon}\left|k^{+}\right|}{\mu_{+}^{2 \epsilon}}\right]\left[\phi_{n, p}^{\gamma} \frac{\left|p^{-}\right|^{\epsilon}}{\mu_{-}^{\epsilon}}\right], \\
O_{\mathrm{II}}^{(0 c)} & =\frac{J^{(0 c)}}{\left(\bar{n} \cdot p n \cdot \ell-i 0^{+}\right)\left(\bar{n} \cdot q n \cdot \ell-i 0^{+}\right)}\left[\phi_{s,-\ell}^{\dagger} \phi_{v}^{b} \frac{\left|\ell^{+}\right|^{\epsilon}}{\mu_{+}^{\epsilon}}\right]\left[\phi_{n, p-q}^{\dagger} \phi_{n,-q} \frac{\left.\left|p^{-}-q^{-}\right|\right|^{\epsilon} \mid q^{-}}{\mu_{-}^{2 \epsilon}}\right],
\end{aligned}
$$

where we have suppressed the sums over label momenta shown in Eq. (91), and in general the $J^{(i)}$ are functions of the label momenta $\left(p^{-}, \ell^{+}, k^{+}, \ldots\right)$. Using the currents $O_{\mathrm{II}}^{(0 b)}$ and $O_{\mathrm{II}}^{(0 c)}$ for the soft and collinear graphs in Figs. 15b,c respectively we have

$$
\begin{aligned}
I_{\mathrm{soft}}^{\text {scalar }} & =\sum_{k^{+} \neq 0} \int \frac{d^{D} k_{r}}{(2 \pi)^{D}} \frac{\mu^{2 \epsilon}}{\left[k^{2}-\ell^{+} k^{-}+i 0^{+}\right]\left[k^{2}-m^{2}+i 0^{+}\right]\left[-p^{-} k^{+}+i 0^{+}\right]} \frac{\left|k^{+}\right| \epsilon\left|k^{+}-\ell^{+}\right| \epsilon}{\mu_{+}^{2 \epsilon}}, \\
I_{\mathrm{cn}}^{\text {scalar }} & =\sum_{k^{-} \neq 0} \int \frac{d^{D} k_{r}^{\prime}}{(2 \pi)^{D}} \frac{\mu^{2 \epsilon}}{\left[-\ell^{+} k^{-}+i 0^{+}\right]\left[k^{2}-m^{2}+i 0^{+}\right]\left[k^{2}-p^{-} k^{+}+i 0^{+}\right]} \frac{\left|k^{-}\right| \epsilon\left|k^{-}-p^{-}\right|^{\epsilon}}{\mu_{-}^{2 \epsilon}} .
\end{aligned}
$$

Here the $k^{+} \neq 0$ and $k^{-} \neq 0$ conditions denote the overlap regions where the soft integration variable becomes collinear and the collinear integration variable becomes soft, as in Fig. 13. The sums over $k^{+} \neq 0$ and $k^{-} \neq 0$ ensure that the $\left[-p^{-} k^{+}\right]$and $\left[-\ell^{+} k^{-}\right]$propagators never get small. By examining the scaling, we find that no subtraction is necessary for $k^{+} \neq \ell^{+}$ and $k^{-} \neq p^{-}$here, so though present, these restrictions were not shown. Eq. (17) tells 
us that unlike the $\mathrm{SCET}_{\mathrm{I}}$ computations and the $\mathrm{SCET}_{\mathrm{II}}$ cutoff computation, here we have zero-bin subtractions for both the soft and collinear diagrams. These will ensure that we do not get spurious singularities from the $\left[-p^{-} k^{+}\right]$and $\left[-\ell^{+} k^{-}\right]$propagators. The naive integrals and subtraction integrals are

$$
\begin{aligned}
\tilde{I}_{\text {soft }}^{\text {scalar }} & =\int \frac{d^{D} k}{(2 \pi)^{D}} \frac{\mu^{2 \epsilon}}{\left[k^{2}-\ell^{+} k^{-}+i 0^{+}\right]\left[k^{2}-m^{2}+i 0^{+}\right]\left[-p^{-} k^{+}+i 0^{+}\right]} \frac{\left|k^{+}\right| \epsilon\left|k^{+}-\ell^{+}\right|^{\epsilon}}{\mu_{+}^{2 \epsilon}}, \\
I_{0 \text { soft }}^{\text {scalar }} & =\int \frac{d^{D} k}{(2 \pi)^{D}} \frac{\mu^{2 \epsilon}}{\left[-\ell^{+} k^{-}+i 0^{+}\right]\left[k^{2}-m^{2}+i 0^{+}\right]\left[-p^{-} k^{+}+i 0^{+}\right]} \frac{\left|k^{+}\right| \epsilon\left|k^{+}-\ell^{+}\right|^{\epsilon}}{\mu_{+}^{2 \epsilon}}, \\
\tilde{I}_{\text {cn }}^{\text {scalar }} & =\int \frac{d^{D} k}{(2 \pi)^{D}} \frac{\mu^{2 \epsilon}}{\left[-\ell^{+} k^{-}+i 0^{+}\right]\left[k^{2}-m^{2}+i 0^{+}\right]\left[k^{2}-p^{-} k^{+}+i 0^{+}\right]} \frac{\left|k^{-}\right| k^{-}-\left.p^{-}\right|^{\epsilon}}{\mu_{-}^{2 \epsilon}}, \\
I_{0 \mathrm{cn}}^{\text {scalar }} & =\int \frac{d^{D} k}{(2 \pi)^{D}} \frac{\left|k^{-}\right| \epsilon\left|k^{-}-p^{-}\right| \epsilon}{\mu_{-}^{2 \epsilon}} .
\end{aligned}
$$

Note that we must keep the $m^{2}$ dependence in the subtraction integrals to properly avoid double counting the zero-bin regions in the differences $\tilde{I}_{\text {soft }}^{\text {scalar }}-I_{0 \text { soft }}^{\text {scalar }}$ and $\tilde{I}_{\text {cn }}^{\text {scalar }}-I_{0 \mathrm{cn}}^{\text {scalar }}$, which from Eq. (17) give the result for $I_{\text {soft }}^{\text {scalar }}$ and $I_{\text {cn }}^{\text {scalar }}$ respectively.

For the soft graph we do the $k^{-}$integral by contours. Due to the pole structure this restricts the $k^{+}$-integration to the region $0<k^{+}<\ell^{+}$. The $k_{\perp}$ integral is then done. For the soft subtraction integral we follow the same procedure which this time leaves the integration region $0<k^{+}<\infty$. We find

$$
\begin{aligned}
\tilde{I}_{\mathrm{soft}}^{\text {scalar }} & =\frac{-i \Gamma(\epsilon) \mu^{2 \epsilon}}{16 \pi^{2}\left(p^{-} \ell^{+}\right)} \int_{0}^{\ell^{+}} \frac{d k^{+}}{k^{+}}\left[\frac{\left(\ell^{+}-k^{+}\right) m^{2}}{\ell^{+}}\right]^{-\epsilon}\left|\frac{k^{+}\left(k^{+}-\ell^{+}\right)}{\mu_{+}^{2}}\right|^{\epsilon} \\
& =\frac{-i \Gamma(\epsilon)}{16 \pi^{2}\left(p^{-} \ell^{+}\right)}\left(\frac{m^{2}}{\mu^{2}}\right)^{-\epsilon}\left(\frac{\ell^{+}}{\mu_{+}}\right)^{2 \epsilon} \frac{1}{\epsilon_{\mathrm{IR}}}, \\
I_{0 \mathrm{soft}}^{\text {scalar }} & =\frac{-i \Gamma(\epsilon) \mu^{2 \epsilon}}{16 \pi^{2}\left(p^{-} \ell^{+}\right)} \int_{0}^{\infty} \frac{d k^{+}}{k^{+}}\left(m^{2}\right)^{-\epsilon}\left|\frac{k^{+}\left(k^{+}-\ell^{+}\right)}{\mu_{+}^{2}}\right|^{\epsilon} \\
& =\frac{-i \Gamma(\epsilon)}{16 \pi^{2}\left(p^{-} \ell^{+}\right)}\left(\frac{m^{2}}{\mu^{2}}\right)^{-\epsilon}\left(\frac{\ell^{+}}{\mu_{+}}\right)^{2 \epsilon}\left(\left\{\frac{1}{\epsilon_{\mathrm{IR}}}-\frac{\pi^{2} \epsilon}{6}\right\}+\left\{-\frac{1}{2 \epsilon_{\mathrm{UV}}}-\frac{\pi^{2} \epsilon}{6}\right\}\right) .
\end{aligned}
$$

In the last line the first $\{\cdots\}$ factor comes from the integral over $0<k^{+}<\ell^{+}$, and the second from $\ell^{+}<k^{+}<\infty$. Computing the full soft integral in Eq. (106), $I_{\text {soft }}^{\text {scalar }}=\tilde{I}_{\text {soft }}^{\text {scalar }}-I_{0 \text { soft }}^{\text {scalar }}$,

$$
\begin{aligned}
& I_{\mathrm{soft}}^{\mathrm{scalar}}= \frac{-i \Gamma(\epsilon)}{16 \pi^{2}\left(p^{-} \ell^{+}\right)}\left(\frac{m^{2}}{\mu^{2}}\right)^{-\epsilon}\left(\frac{\ell^{+}}{\mu_{+}}\right)^{2 \epsilon}\left(\frac{1}{2 \epsilon_{\mathrm{UV}}}+\frac{\pi^{2} \epsilon}{3}\right) \\
&=\frac{-i}{16 \pi^{2}\left(p^{-} \ell^{+}\right)}\left[\frac{1}{2 \epsilon_{\mathrm{UV}}^{2}}+\frac{1}{\epsilon_{\mathrm{UV}}} \ln \left(\frac{\ell^{+}}{\mu_{+}}\right)-\frac{1}{2 \epsilon_{\mathrm{UV}}} \ln \left(\frac{m^{2}}{\mu^{2}}\right)+\ln ^{2}\left(\frac{\ell^{+}}{\mu_{+}}\right)+\frac{3 \pi^{2}}{8}\right. \\
&\left.\quad+\frac{1}{4} \ln ^{2}\left(\frac{m^{2}}{\mu^{2}}\right)-\ln \left(\frac{m^{2}}{\mu^{2}}\right) \ln \left(\frac{\ell^{+}}{\mu_{+}}\right)\right] .
\end{aligned}
$$

Much like the examples in $\mathrm{SCET}_{\mathrm{I}}$ the zero-bin subtraction integral $I_{0 \text { soft }}^{\text {scalar }}$ cancels the IR singularity in the $k^{+}$integration in $\tilde{I}_{\text {soft }}^{\text {scalar }}$ and replaces it by a UV divergence. 
For the collinear integrals, we do the contour integration in $k^{+}$which restricts the remaining integration region in $k^{-}$. For the naive and subtraction integrals we find

$$
\begin{aligned}
\tilde{I}_{\mathrm{cn}}^{\text {scalar }} & =\frac{-i \Gamma(\epsilon) \mu^{2 \epsilon}}{16 \pi^{2}\left(p^{-} \ell^{+}\right)} \int_{0}^{p^{-}} \frac{d k^{-}}{k^{-}}\left[\frac{\left(p^{-}-k^{-}\right) m^{2}}{p^{-}}\right]^{-\epsilon}\left|\frac{k^{-}\left(k^{-}-p^{-}\right)}{\mu_{-}^{2}}\right|^{\epsilon} \\
I_{0 \mathrm{cn}}^{\text {scalar }} & =\frac{-i \Gamma(\epsilon) \mu^{2 \epsilon}}{16 \pi^{2}\left(p^{-} \ell^{+}\right)} \int_{0}^{\infty} \frac{d k^{-}}{k^{-}}\left(m^{2}\right)^{-\epsilon}\left|\frac{k^{-}\left(k^{-}-p^{-}\right)}{\mu_{-}^{2}}\right|^{\epsilon},
\end{aligned}
$$

which for our example, are the same integrals as for the soft loops but with $\ell^{+} \rightarrow p^{-}$and $\mu_{+} \rightarrow \mu_{-}$. Thus for the complete collinear result in Eq. (106), $I_{\text {cn }}^{\text {scalar }}=\tilde{I}_{\text {cn }}^{\text {scalar }}-I_{0 \mathrm{cn}}^{\text {scalar }}$, we find

$$
\begin{aligned}
I_{\mathrm{cn}}^{\mathrm{scalar}=} & \frac{-i \Gamma(\epsilon)}{16 \pi^{2}\left(p^{-} \ell^{+}\right)}\left(\frac{m^{2}}{\mu^{2}}\right)^{-\epsilon}\left(\frac{p^{-}}{\mu_{-}}\right)^{2 \epsilon}\left(\frac{1}{2 \epsilon_{\mathrm{UV}}}+\frac{\pi^{2} \epsilon}{3}\right) \\
= & \frac{-i}{16 \pi^{2}\left(p^{-} \ell^{+}\right)}\left[\frac{1}{2 \epsilon_{\mathrm{UV}}^{2}}+\frac{1}{\epsilon_{\mathrm{UV}}} \ln \left(\frac{p^{-}}{\mu_{-}}\right)-\frac{1}{2 \epsilon_{\mathrm{UV}}} \ln \left(\frac{m^{2}}{\mu^{2}}\right)+\ln ^{2}\left(\frac{p^{-}}{\mu_{-}}\right)+\frac{3 \pi^{2}}{8}\right. \\
& \left.+\frac{1}{4} \ln ^{2}\left(\frac{m^{2}}{\mu^{2}}\right)-\ln \left(\frac{m^{2}}{\mu^{2}}\right) \ln \left(\frac{p^{-}}{\mu_{-}}\right)\right] .
\end{aligned}
$$

The results in Eqs. (109) and (111) have $1 / \epsilon_{\mathrm{UV}} \ln \left(\mathrm{m}^{2}\right)$ divergences, terms that did not appear in our example with a rapidity cutoff, and are simply artifacts of the dimensional regularization setup. These divergences arise from the fact that the UV collinear divergences induced by the zero-bin subtraction are multiplicative over all loops and propagators. They are canceled by a special type of counterterm that is proportional to the renormalized distribution function at the origin, $\phi(0, \mu) / \epsilon_{\mathrm{UV}}$. The presence of these operators is discussed further in section VIIG below. Here we have one such counterterm current for the soft loop and one for the collinear loop

$$
\begin{aligned}
O_{\mathrm{II}}^{(0 d)} & =\left.\sum_{p^{-}, \ell^{+} \neq 0} \frac{J^{(0 d)}}{\left[\left(\bar{n} \cdot p n \cdot \ell-i 0^{+}\right) \bar{n} \cdot p\right]}\left[\phi_{s,-k}^{\dagger} \phi_{v}^{b} \phi_{s, \ell}^{g}\right]\left[\phi_{n, p}^{\gamma}\right]\right|_{k^{+} \rightarrow 0}, \\
O_{\mathrm{II}}^{(0 e)} & =\left.\sum_{p^{-}, \ell^{+} \neq 0} \frac{J^{(0 e)}}{\left[\left(\bar{n} \cdot p n \cdot \ell-i 0^{+}\right) n \cdot \ell\right]}\left[\phi_{s,-\ell}^{\dagger} \phi_{v}^{b}\right]\left[\phi_{n, p}^{\dagger} \phi_{n,-q}\right]\right|_{q^{-} \rightarrow 0}
\end{aligned} .
$$

These correspond to counterterms for operators which give a $\phi(0, \mu)$, corresponding to the initial $B$ meson, and a $\phi(0, \mu)$ for the quark part of the final photon wavefunction. Note that the limit $k^{+} \rightarrow 0$ and $q^{-} \rightarrow 0$ is done at the end.

The necessary counterterm coefficients for the results in Eqs. (109) and (111) are

$$
\delta J^{(0 d)}=\frac{e g G}{2 \epsilon_{\mathrm{UV}}}, \quad \delta J^{(0 e)}=\frac{e g G}{2 \epsilon_{\mathrm{UV}}} .
$$

At one loop these operator generate graphs similar to the diagrams in Fig. 15]b,c. Using the same IR mass regulator, pulling out the same prefactor as the other diagrams, and 
performing the standard UV renormalization of the operators in $\overline{\mathrm{MS}}$ prior to multiplying by the rapidity counterterm gives

$$
\begin{aligned}
& I_{\mathrm{ct} 0 \mathrm{~d}}^{\mathrm{scalar}}=\left(\frac{1}{2 \epsilon_{\mathrm{UV}}}\right) \frac{i}{16 \pi^{2}\left(p^{-} \ell^{+}\right)}\left[-\ln \left(\frac{m^{2}}{\mu^{2}}\right)\right], \\
& I_{\mathrm{ct} 0 \mathrm{e}}^{\mathrm{scalar}}=\left(\frac{1}{2 \epsilon_{\mathrm{UV}}}\right) \frac{i}{16 \pi^{2}\left(p^{-} \ell^{+}\right)}\left[-\ln \left(\frac{m^{2}}{\mu^{2}}\right)\right] .
\end{aligned}
$$

Due to our choice of $\delta J^{(0 d, 0 e)}$ these counterterm diagrams exactly cancel the $1 / \epsilon_{\mathrm{UV}} \ln \left(m^{2}\right)$ terms in the collinear and soft loops. In dimensional regularization, this type of counterterm operator is quite important to the rapidity renormalization, as we discuss in the next section. In particular in going beyond perturbation theory, these same type of counterterms are required to cancel UV divergences in the convolution over the non-perturbative matrix element.

Adding the soft, collinear, and $I_{\mathrm{ct} 0 \mathrm{~d}}^{\text {scalar }}, I_{\mathrm{ct} 0 \mathrm{e}}^{\text {scalar }}$ counterterms we find the $\mathrm{SCET}_{\mathrm{II}}$ result

$$
\begin{aligned}
I_{\mathrm{soft}+\mathrm{cn}}^{\mathrm{scalar}}= & \frac{-i}{16 \pi^{2}\left(p^{-} \ell^{+}\right)}\left[\frac{1}{2} \ln ^{2}\left(\frac{m^{2}}{\mu^{2}}\right)-\ln \left(\frac{m^{2}}{\mu^{2}}\right) \ln \left(\frac{p^{-}}{\mu_{-}}\right)-\ln \left(\frac{m^{2}}{\mu^{2}}\right) \ln \left(\frac{\ell^{+}}{\mu_{+}}\right)\right. \\
& \left.+\frac{1}{\epsilon_{\mathrm{UV}}^{2}}+\frac{1}{\epsilon_{\mathrm{UV}}} \ln \left(\frac{p^{-} \ell^{+}}{\mu_{-} \mu_{+}}\right)+\ln ^{2}\left(\frac{p^{-}}{\mu_{-}}\right)+\ln ^{2}\left(\frac{\ell^{+}}{\mu_{+}}\right)+\frac{3 \pi^{2}}{4}\right] \\
= & \frac{-i}{16 \pi^{2}\left(p^{-} \ell^{+}\right)}\left[\frac{1}{2} \ln ^{2}\left(\frac{m^{2}}{p^{-} \ell^{+}}\right)-\ln \left(\frac{m^{2}}{\mu^{2}}\right) \ln \left(\frac{\mu^{2}}{\mu_{-} \mu_{+}}\right)\right. \\
& \left.+\frac{1}{\epsilon_{\mathrm{UV}}^{2}}+\frac{1}{\epsilon_{\mathrm{UV}}} \ln \left(\frac{p^{-} \ell^{+}}{\mu_{-} \mu_{+}}\right)+\ln ^{2}\left(\frac{p^{-}}{\mu_{-}}\right)+\ln ^{2}\left(\frac{\ell^{+}}{\mu_{+}}\right)-\frac{1}{2} \ln ^{2}\left(\frac{p^{-} \ell^{+}}{\mu^{2}}\right)+\frac{3 \pi^{2}}{4}\right] .
\end{aligned}
$$

The effective theory still has UV divergences shown on the second line of Eq. (115). These divergences occur because of the separation of $\zeta_{p}$ momenta. The remaining UV divergences are canceled by a counterterm for the jet function coefficient $J^{(0 a)}$. Putting back the prefactor, we find the counterterm

$$
\delta J^{(0 a)}=\frac{e g^{2} G}{16 \pi^{2}\left(p^{-} \ell^{+}\right)}\left[-\frac{1}{\epsilon_{\mathrm{UV}}^{2}}-\frac{1}{\epsilon_{\mathrm{UV}}} \ln \left(\frac{p^{-} \ell^{+}}{\mu_{-} \mu_{+}}\right)\right] .
$$

As is familiar from $\mathrm{SCET}_{\mathrm{I}}$, the counterterm depends on logs involving $\mu$ 's. Thus, finally, the renormalized EFT result is

$$
\begin{aligned}
I_{\mathrm{soft}+\mathrm{cn}}^{\text {scalar }}=\frac{-i}{16 \pi^{2}\left(p^{-} \ell^{+}\right)} & {\left[\frac{1}{2} \ln ^{2}\left(\frac{m^{2}}{p^{-} \ell^{+}}\right)-\ln \left(\frac{m^{2}}{\mu^{2}}\right) \ln \left(\frac{\mu^{2}}{\mu_{-} \mu_{+}}\right)\right.} \\
+ & \left.\ln ^{2}\left(\frac{p^{-}}{\mu_{-}}\right)+\ln ^{2}\left(\frac{\ell^{+}}{\mu_{+}}\right)-\frac{1}{2} \ln ^{2}\left(\frac{p^{-} \ell^{+}}{\mu^{2}}\right)+\frac{3 \pi^{2}}{4}\right] .
\end{aligned}
$$

Comparing the first two terms in Eq. (117) with the full theory result in Eq. (104) we see that the IR divergences are exactly reproduced if and only if

$$
\mu^{2}=\mu_{+} \mu_{-}
$$


Thus again this condition follows from the dynamics. It is interesting to note that the $\ln \left(p^{-} \ell^{+}\right) \ln \left(m^{2}\right)$ divergence is reproduced independent of the power of $|\overline{\mathcal{P}}|$ and $|\mathcal{P}|$ used in Eq. (103), but that the $\ln ^{2}\left(m^{2}\right)$ term is only reproduced for the power $\epsilon$, which was derived in section VIIB. Using $\mu^{2}=\mu_{+} \mu_{-}$, the difference of the remaining finite terms gives a contribution to the one-loop matching

$$
\begin{aligned}
I_{\text {match }}^{\text {scalar }} & =\frac{-i}{16 \pi^{2}\left(p^{-} \ell^{+}\right)}\left[\frac{1}{2} \ln ^{2}\left(\frac{p^{-} \ell^{+}}{\mu_{-} \mu_{+}}\right)-\ln ^{2}\left(\frac{p^{-}}{\mu_{-}}\right)-\ln ^{2}\left(\frac{\ell^{+}}{\mu_{+}}\right)-\frac{5 \pi^{2}}{12}\right] \\
& =\frac{-i}{16 \pi^{2}\left(p^{-} \ell^{+}\right)}\left[-\frac{1}{2} \ln ^{2}\left(\frac{p^{-} \mu_{+}}{\mu_{-} \ell^{+}}\right)-\frac{5 \pi^{2}}{12}\right] .
\end{aligned}
$$

From Eq. (119) we see that the jet function will be a non-trivial function of $\mu_{+}$and $\mu_{-}$ (and thus $\mu^{2}=\mu_{+} \mu_{-}$) whose $\mu$-dependences will cancel against dependence on these variables in the $\mathrm{SCET}_{\mathrm{II}}$ matrix elements. The matching coefficient arises from integrating out perturbative effects associated with $p^{-} l^{+}$as well as perturbative effects responsible for the rapidity gap between the soft and collinear modes, and therefore has a different structure than what would be obtained if only the former were integrated out. In Appendix B we verify that the same result for $I_{\text {match }}^{\text {scalar }}$ is obtained when we regulate the IR divergences in the full and effective theories with three non-equal masses, $m_{1,2,3}$, or when we keep the $\ell^{2}$ and $p^{2}$ offshell. The result should be the same because the matching only depends on the UV regulator which we keep the same in these computations. Note that in Eq. (102) we used a different UV regulator than in Eq. (119), which explains why the $\pi^{2}$ terms differ.

The results in Eq. (116) and (119) are shown for illustration only, since of course, the complete anomalous dimension and matching calculations require a computation of all diagrams, not just the one diagram that we considered here for illustration.

\section{Zero-Bin Subtractions in Convolutions: General Discussion}

The remaining application of subtractions in $\mathrm{SCET}_{\mathrm{II}}$ will be for factorization formulas which appear to suffer from singular convolutions at the level of tree level matching. Much like the example discussed in $\mathrm{SCET}_{\mathrm{I}}$, in $\mathrm{SCET}_{\mathrm{II}}$ we must avoid the zero-bin in hard scattering kernels defined by tree level matching. Doing so removes the double counting problem and renders singular convolutions finite. Here we only deal with the rapidity renormalization, so we make the simplifying assumption that the standard UV divergences have already been taken care of, and do not interfere with the steps carried out here.

We will use dimensional regularization to separate the modes in rapidity space as in Eq. (103). To see why the convolution integrals are always finite, let us consider the vacuum to pion matrix element of a hard scattering kernel $J\left(p_{i}^{-}, \mu_{-}, \mu^{2}\right)$ and a twist-2 collinear operator in $\operatorname{SCET}_{\mathrm{II}}$ that gives the light-cone distribution function $\phi_{\pi}(x, \mu)$. For simplicity 
we will not write the $\mu$-dependences for $J$ and $\phi_{\pi}$ below. ${ }^{22}$ This leaves the matrix element

$$
\begin{aligned}
A_{\pi} & =\sum_{p_{1,2}^{-} \neq 0} \int d p_{1 r}^{-} d p_{2 r}^{-} J\left(p_{1}^{-}, p_{2}^{-}\right)\left\langle\pi_{n}\left(p_{\pi}\right)\left|\left(\bar{\xi}_{n} W\right)_{p_{1}^{-}} \not h \gamma_{5}\left(W^{\dagger} \xi_{n}\right)_{-p_{2}^{-}}\right| 0\right\rangle\left|\frac{p_{1}^{-} p_{2}^{-}}{\mu_{-}^{2}}\right|^{\epsilon} \\
& =-i f_{\pi} \sum_{p_{1,2}^{-} \neq 0} \int d p_{1 r}^{-} d p_{2 r}^{-} J\left(p_{1}^{-}, p_{2}^{-}\right) \delta\left(\bar{n} \cdot p_{\pi}-p_{1}^{-}-p_{2}^{-}\right) \phi_{\pi}\left(x_{1}, x_{2}\right)\left|\frac{p_{1}^{-} p_{2}^{-}}{\mu_{-}^{2}}\right|^{\epsilon} \\
& =-i f_{\pi} \bar{n} \cdot p_{\pi}\left(\frac{\bar{n} \cdot p_{\pi}}{\mu_{-}}\right)^{2 \epsilon} \sum_{x_{1,2} \neq 0} \int d x_{1 r} d x_{2 r} J\left(x_{1}, x_{2}\right) \delta\left(1-x_{1}-x_{2}\right) \phi_{\pi}\left(x_{1}, x_{2}\right)\left|x_{1} x_{2}\right|^{\epsilon},
\end{aligned}
$$

where we switched to dimensionless variables $x_{1,2}$ via $p_{1}^{-}=x_{1} \bar{n} \cdot p_{\pi}$ and $p_{2}^{-}=x_{2} \bar{n} \cdot p_{\pi}$, and in the second line we inserted the standard definition of the twist- 2 distribution function

$$
\left\langle\pi_{n}^{+}\left(p_{\pi}\right)\left|\bar{u}_{n, p_{1}^{-}} \not h \gamma_{5} d_{n,-p_{2}^{-}}\right| 0\right\rangle=-i f_{\pi} \delta\left(\bar{n} \cdot p_{\pi}-p_{1}^{-}-p_{2}^{-}\right) \phi_{\pi}\left(x_{1}, x_{2}, \mu\right),
$$

where the $\delta$ function gives conservation of momentum. Now suppose that we computed $J$ at tree level (by a matching computation) and found that $J\left(x_{1}, x_{2}\right)=1 /\left(p_{1}^{-}\right)^{2}=1 /\left[\left(\bar{n} \cdot p_{\pi}\right)^{2} x_{1}^{2}\right]$. If we were not careful about the $x_{1} \neq 0$ condition, this would lead to a singular convolution integral as in Eq. (2). The zero-bin subtraction formula in Eq. (17) tells us to impose the momentum conserving $\delta$-functions carrying through all zero-bin constraints. Since the $x_{2^{-}}$ integration is not singular, there are no zero-bin subtractions for $x_{2} \neq 0$ and we can combine the sum over label $x_{2}$ momenta and integral over residual $x_{2 r}$ momenta back into a integral over all $x_{2}$ momenta using Eq. (16):

$$
\begin{aligned}
A_{\pi} & =-i \frac{f_{\pi}}{\bar{n} \cdot p_{\pi}}\left(\frac{\bar{n} \cdot p_{\pi}}{\mu_{-}}\right)^{2 \epsilon} \sum_{x_{1} \neq 0} \int d x_{1 r} d x_{2} \frac{1}{\left(x_{1}\right)^{2}} \delta\left(1-x_{1}-x_{2}\right) \phi_{\pi}\left(x_{1}, x_{2}\right)\left|x_{1} x_{2}\right|^{\epsilon} \\
& =-i \frac{f_{\pi}}{\bar{n} \cdot p_{\pi}}\left(\frac{\bar{n} \cdot p_{\pi}}{\mu_{-}}\right)^{2 \epsilon} \sum_{x_{1} \neq 0} \int d x_{1 r} \frac{1}{\left(x_{1}\right)^{2}} \theta\left(1-x_{1}\right) \theta\left(x_{1}\right) \hat{\phi}_{\pi}\left(x_{1}\right)\left|x_{1}\left(1-x_{1}\right)\right|^{\epsilon} .
\end{aligned}
$$

where $\bar{x}_{1}=1-x_{1}$. If there had been zero-bin subtractions for $x_{2}$ they would carry through as additional zero-bin subtractions at $x_{1} \neq 1$ after removing the $\delta$-function. In the last line we set $\phi_{\pi}\left(x_{1}, \bar{x}_{1}\right)=\theta\left(1-x_{1}\right) \theta\left(x_{1}\right) \hat{\phi}_{\pi}\left(x_{1}\right)$ to make the support of the non-perturbative distribution function explicit. To turn the final sum over labels and integral over residual momenta into an integral over $x_{1}$, there will be zero-bin subtractions from Eq. (17). The subtraction acts on the integrand including the $\theta$-functions, but just as in our perturbative analysis, it does not act on the $\left|x_{1}\left(1-x_{1}\right)\right|^{\epsilon}$ factor. The expansion for $x_{1} \neq 0$ is from the right, about $x_{1}=0^{+}$, since this is how the variable scales towards the zero-bin region:

$$
\begin{aligned}
\theta\left(1-x_{1}\right) \theta\left(x_{1}\right) \hat{\phi}_{\pi}\left(x_{1}\right)= & \theta\left(x_{1}\right)\left[\hat{\phi}_{\pi}(0)+x_{1} \hat{\phi}_{\pi}^{\prime}(0)+\frac{x_{1}^{2}}{2} \hat{\phi}_{\pi}^{\prime \prime}(0)+\ldots\right] \\
& +\theta\left(x_{1}\right) \hat{\phi}_{\pi}(0)\left[\delta\left(1-x_{1}\right)+\ldots\right]
\end{aligned}
$$

${ }^{22}$ Note that integer powers of the $p_{i}^{-}$can be moved from $J$ to the collinear operator by inserting powers of $\overline{\mathcal{P}}$, but that our analysis is independent of this freedom. 
In the set of terms obtained on the first line, the $\theta\left(1-x_{1}\right)$ disappears in the series so the support of the $x_{1}$ integration for the subtraction terms differs from that for the naive integral. This is the same as what we saw in our perturbation theory example in Eq. (110), where the naive integral was integrated over $k^{-} \in\left[0, p^{-}\right]$, ie. $x_{1} \in[0,1]$, but the the subtraction integral was integrated over $k^{-} \in[0, \infty]$, ie. $x_{1} \in[0, \infty]$. In the last line in Eq. (123), the terms are all zero (or finite subtractions) for the cases considered here, and therefore these terms do not contribute for our choice of zero-bin scheme as discussed in section $\mathbb{I V}$.

Lets make the standard assumption for the twist-2 distribution that $\phi_{\pi}(0)=0$. Then using Eq. (17), the result for $A_{\pi}$ is

$$
A_{\pi}=\frac{-i f_{\pi}}{\bar{n} \cdot p_{\pi}}\left(\frac{p_{\pi}^{-}}{\mu_{-}}\right)^{2 \epsilon} \int d x_{1} \frac{\theta\left(x_{1}\right)}{\left(x_{1}\right)^{2}}\left[\theta\left(1-x_{1}\right) \hat{\phi}_{\pi}\left(x_{1}\right)-x_{1} \hat{\phi}_{\pi}^{\prime}(0)\right]\left|x_{1}\left(1-x_{1}\right)\right|^{-\epsilon},
$$

where as usual only the subtraction needed to remove the singular term was kept. Next we split the integration into a finite integral $x_{1} \in[0,1]$ where the factor of $\left|x_{1}\left(1-x_{1}\right)\right|^{\epsilon}$ can be set to 1 , and the integral of the subtraction term over $x_{1} \in[1, \infty]$ where the $\epsilon$ dependent term is needed

$$
\begin{aligned}
A_{\pi} & =-i \frac{f_{\pi}}{\bar{n} \cdot p_{\pi}}\left(\frac{\bar{n} \cdot p_{\pi}}{\mu_{-}}\right)^{2 \epsilon}\left\{\int_{0}^{1} d x_{1} \frac{\phi_{\pi}\left(x_{1}\right)-x_{1} \phi_{\pi}^{\prime}(0)}{\left(x_{1}\right)^{2}}-\int_{1}^{\infty} d x_{1} \frac{x_{1}^{\epsilon}\left(x_{1}-1\right)^{\epsilon}}{\left(x_{1}\right)^{2}}\left[x_{1} \phi_{\pi}^{\prime}(0)\right]\right\} \\
& =-i \frac{f_{\pi}}{\bar{n} \cdot p_{\pi}}\left(\frac{\bar{n} \cdot p_{\pi}}{\mu_{-}}\right)^{2 \epsilon}\left\{\int_{0}^{1} d x_{1} \frac{\phi_{\pi}\left(x_{1}\right)-x_{1} \phi_{\pi}^{\prime}(0)}{\left(x_{1}\right)^{2}}+\frac{1}{2 \epsilon_{\mathrm{UV}}} \phi_{\pi}^{\prime}(0)\right\} .
\end{aligned}
$$

Here terms of $\mathcal{O}(\epsilon)$ have been dropped.

Eq. (125) is UV divergent, but we must still add to it the pion matrix element of the counterterm operator. This operator is determined by the UV counterterms that are necessary to renormalize our original operator, and can be derived in perturbation theory with any desired external states. Carrying out a one-loop computation with external quark states and using our perturbative kernel $J=1 /\left(p_{1}^{-}\right)^{2}$ we find the counterterm operator

$$
O_{c t}^{[1]}=\left.C_{c t}^{[1]} \int d p_{2}^{-}\left[\frac{\partial}{\partial p_{1}^{-}}-\frac{\partial}{\partial\left(p_{1}^{-}+p_{2}^{-}\right)}\right]\left(\bar{\xi}_{n} W\right)_{p_{1}^{-}} \not h \gamma_{5}\left(W^{\dagger} \xi_{n}\right)_{-p_{2}^{-}}\right|_{p_{1}^{-} \rightarrow 0},
$$

with a counterterm coefficient $\delta C_{c t}^{[1]}=-1 /\left(2 \epsilon_{\mathrm{UV}}\right)$. The derivative with respect to $\left(p_{1}^{-}+p_{2}^{-}\right)$ removes surface terms. In the vacuum to pion matrix element, they would result from a $d / d p_{1}^{-}$of the $\delta$-function in Eq. (121) if we had left out the $d / d\left(p_{1}^{-}+p_{2}^{-}\right)$. At tree level with quarks the matrix element of this operator vanishes — one obtains $\delta^{\prime}\left(p^{-}\right)$factors and the quark states have non-zero $p^{-}$momenta. The vacuum to pion matrix element of $O_{c t}^{[1]}$ gives

$$
\begin{aligned}
A_{\pi}^{c t 1} & =-\left.\frac{1}{2 \epsilon_{\mathrm{UV}}} \int d p_{2}^{-}\left[\frac{d}{d p_{1}^{-}}+\frac{d}{d p_{\pi}^{-}}\right]\left\langle\pi_{n}\left(p_{\pi}\right)\left|\left(\bar{\xi}_{n} W\right)_{p_{1}^{-}} \not h \gamma_{5}\left(W^{\dagger} \xi_{n}\right)_{-p_{2}^{-}}\right| 0\right\rangle\right|_{p_{1}^{-} \rightarrow 0} \\
& =\left.\frac{i f_{\pi}}{2 \epsilon_{\mathrm{UV}} p_{\pi}^{-}} \int d p_{2}^{-} \delta\left(\bar{n} \cdot p_{\pi}-p_{1}^{-}-p_{2}^{-}\right) \phi_{\pi}^{(1,0)}\left(x_{1}, x_{2}, \mu\right)\right|_{p_{1}^{-} \rightarrow 0} \\
& =i \frac{f_{\pi}}{\bar{n} \cdot p_{\pi}} \frac{1}{2 \epsilon_{\mathrm{UV}}} \phi_{\pi}^{\prime}(0),
\end{aligned}
$$


where the superscript $(1,0)$ indicates a derivative with respect to the first argument. We should also include the matrix element of the finite part of the counterterm operator in Eq. (126) 91, which gives

$$
A_{\pi}^{c t 2}=-i \frac{f_{\pi}}{\bar{n} \cdot p_{\pi}} C_{c t}^{[1]}\left(\mu_{-}\right) \phi_{\pi}^{\prime}(0) .
$$

Adding the $A_{\pi}^{c t 1}$ term to Eq. (125), the UV divergence cancels, and sending $\epsilon \rightarrow 0$ we obtain the finite result

$$
\begin{aligned}
A_{\pi}+A_{\pi}^{c t 1} & =-i \frac{f_{\pi}}{\bar{n} \cdot p_{\pi}}\left\{\int_{0}^{1} d x_{1} \frac{\phi_{\pi}\left(x_{1}, \mu\right)-x_{1} \phi_{\pi}^{\prime}(0, \mu)}{\left(x_{1}\right)^{2}}+\phi_{\pi}^{\prime}(0, \mu) \ln \left(\frac{\bar{n} \cdot p_{\pi}}{\mu_{-}}\right)\right\} \\
& \equiv-i \frac{f_{\pi}}{\bar{n} \cdot p_{\pi}} \int_{0}^{1} d x_{1} \frac{\phi_{\pi}\left(x_{1}, \mu, \mu_{-}\right)}{\left(x_{1}^{2}\right)_{\varnothing}} .
\end{aligned}
$$

As indicated, performing the steps outlined from Eq. (120) to (129) defines the $\varnothing$-distribution in dimensional regularization with our renormalization scheme. The $\mu_{-}$in the distribution, $\phi\left(x_{1}, \mu, \mu_{-}\right)$is a short hand for the dependence on the $\ln \left(\mu_{-}\right)$in the first line of Eq. (129). Once again, in Eq. (129) the zero-bin subtraction has converted an IR divergence into a UV divergence - the naive IR divergence in the convolution has been converted into a UV divergence for the operator in Eq. (121), which is canceled by the operator renormalization counterterm in Eq. (126). Essentially the $\varnothing$-distribution notation on a variable, $(x)_{\varnothing}$ indicates that we have a sum over labels $x \neq 0$, and do an integral over residuals $d x_{r}$, together with applying the rapidity renormalization procedure outlined above for the UV divergences. The $\mu_{-}$dependence in Eq. (129) is canceled by $C_{c t}^{[1]}\left(\mu_{-}\right)$[91] in Eq. (128).

For other cases, the steps in determining the result for the $\varnothing$-distribution are the same as in our example; however it should be clear that the final result will depend on how singular the perturbative kernel is, as well as the endpoint properties of the non-perturbative function that the $\varnothing$-distribution is acting on. In particular, if the starting kernel was not singular there would be no zero-bin subtractions and we would obtain the naive result for the convolution that one finds without the $\varnothing$-distribution. Note that if we had implemented a hard cutoff as in section VIIA 1 rather than dimensional regularization, then lower limits, like $x \geq \delta$, would be induced on the convolution integrals, together with compensating $\delta$ dependence in the jet functions.

For illustration, we consider a few other cases in dimensional regularization that are quite common and which appear in the examples in the next section. First consider a distribution $\phi_{\pi}^{p}\left(x_{1}, x_{2}, \mu\right)$ that does not vanish at its endpoints, integrated against a kernel $J=1 /\left(\bar{n} \cdot p_{\pi} p_{1}^{-}\right)$. For the analog of Eq. (121) we take the matrix element of the operator to give $-i f_{\pi} \mu_{\pi} \delta\left(\bar{n} \cdot p_{\pi}-p_{1}^{-}-p_{2}^{-}\right) \phi_{\pi}^{p}\left(x_{1}, x_{2}, \mu\right)$ where $\mu_{\pi}=m_{\pi}^{2} /\left(m_{u}+m_{d}\right)$, using Eq. (142) 
below with $n \leftrightarrow \bar{n}$. The steps leading up to Eq. (122) are very similar, giving

$$
\begin{aligned}
B_{\pi} & =-i \frac{f_{\pi} \mu_{\pi}}{\bar{n} \cdot p_{\pi}}\left(\frac{\bar{n} \cdot p_{\pi}}{\mu_{-}}\right)^{2 \epsilon} \sum_{x_{1} \neq 0} \int d x_{1 r} \frac{1}{\left(x_{1}\right)} \theta\left(1-x_{1}\right) \theta\left(x_{1}\right) \hat{\phi}_{\pi}^{p}\left(x_{1}\right)\left|x_{1}\left(1-x_{1}\right)\right|^{\epsilon} \\
& =-i \frac{f_{\pi} \mu_{\pi}}{\bar{n} \cdot p_{\pi}}\left(\frac{\bar{n} \cdot p_{\pi}}{\mu_{-}}\right)^{2 \epsilon} \int d x_{1} \frac{\theta\left(x_{1}\right)}{\left(x_{1}\right)}\left[\theta\left(1-x_{1}\right) \hat{\phi}_{\pi}^{p}\left(x_{1}\right)-\hat{\phi}_{\pi}^{p}(0)\right]\left|x_{1}\left(1-x_{1}\right)\right|^{\epsilon} \\
& =-i \frac{f_{\pi} \mu_{\pi}}{\bar{n} \cdot p_{\pi}}\left(\frac{\bar{n} \cdot p_{\pi}}{\mu_{-}}\right)^{2 \epsilon}\left\{\int_{0}^{1} d x_{1} \frac{\phi_{\pi}^{p}\left(x_{1}\right)-\phi_{\pi}^{p}(0)}{x_{1}}-\int_{1}^{\infty} d x_{1} \frac{x_{1}^{\epsilon}\left(x_{1}-1\right)^{\epsilon}}{x_{1}} \phi_{\pi}^{p}(0)\right\} \\
& =-i \frac{f_{\pi} \mu_{\pi}}{\bar{n} \cdot p_{\pi}}\left(\frac{\bar{n} \cdot p_{\pi}}{\mu_{-}}\right)^{2 \epsilon}\left\{\int_{0}^{1} d x_{1} \frac{\phi_{\pi}^{p}\left(x_{1}\right)-\phi_{\pi}^{p}(0)}{x_{1}}+\frac{1}{2 \epsilon_{\mathrm{UV}}} \phi_{\pi}^{p}(0)\right\} .
\end{aligned}
$$

For the zero-bin subtraction in the second line we kept the first term in the analog of the expansion in Eq. (123). Here the counterterm operator is

$$
O_{c t}^{[0]}=\left.C_{c t}^{[0]} \int d p_{2}^{-}\left(\bar{\xi}_{n} W\right)_{p_{1}^{-}} \frac{\not h \gamma_{5}}{2}\left\{\frac{1}{\overline{\mathcal{P}}^{2}}\left(i \mathcal{D}_{n}^{\perp}\right)-\left(i \mathcal{D}_{n}^{\perp}\right)^{\dagger} \frac{1}{\overline{\mathcal{P}}^{\dagger}}\right\}\left(W \xi_{n}\right)_{-p_{2}^{-}} \frac{1}{\overline{\mathcal{P}}^{\dagger}}\right|_{p_{1}^{-} \rightarrow 0},
$$

with $\delta C_{c t}=-1 /\left(2 \epsilon_{\mathrm{UV}}\right)$. The tree level quark matrix element of this operator vanishes. Eq. (131) with $\delta C_{c t}$ gives a vacuum to pion matrix element

$$
\begin{aligned}
B_{\pi}^{c t 1} & =\left.\frac{i f_{\pi} \mu_{\pi}}{2 \epsilon_{\mathrm{UV}} p_{\pi}^{-}} \int d p_{2}^{-}\left[\delta\left(\bar{n} \cdot p_{\pi}-p_{1}^{-}-p_{2}^{-}\right) \phi_{\pi}^{p}\left(x_{1}, x_{2}, \mu\right)\right]\right|_{p_{1}^{-} \rightarrow 0} \\
& =\frac{i f_{\pi} \mu_{\pi}}{\bar{n} \cdot p_{\pi}} \frac{1}{2 \epsilon_{\mathrm{UV}}} \phi_{\pi}^{p}(0) .
\end{aligned}
$$

This term cancels the UV divergence in Eq. (130) to leave the finite result

$$
\begin{aligned}
B_{\pi}+B_{\pi}^{c t 1} & =-i \frac{f_{\pi} \mu_{\pi}}{\bar{n} \cdot p_{\pi}}\left\{\int_{0}^{1} d x_{1} \frac{\phi_{\pi}^{p}\left(x_{1}, \mu\right)-\phi_{\pi}^{p}(0, \mu)}{x_{1}}+\ln \left(\frac{\bar{n} \cdot p_{\pi}}{\mu_{-}}\right) \phi_{\pi}^{p}(0, \mu)\right\} \\
& \equiv-i \frac{f_{\pi} \mu_{\pi}}{\bar{n} \cdot p_{\pi}} \int_{0}^{1} d x_{1} \frac{\phi_{\pi}^{p}\left(x_{1}, \mu, \mu_{-}\right)}{\left(x_{1}\right)_{\varnothing}}
\end{aligned}
$$

As indicated the result in the first line defines the $\varnothing$-distribution for this case. Again we should add to this the matrix element of $O_{c t}^{[0]}$ with the $C_{c t}^{[0]}\left(\mu_{-}\right)$coefficient.

We also will need results for distributions like $\phi_{\pi}^{p}$ and $\phi_{\pi}$ but with zero-bin subtractions 
at both ends of the integration regions

$$
\begin{aligned}
\int d x & d y \frac{\phi_{\pi}^{p}(x, y)}{x_{\varnothing} y_{\varnothing}} \delta(1-x-y) \\
= & \left(\frac{\bar{n} \cdot p_{\pi}}{\mu_{-}}\right)^{2 \epsilon} \sum_{x \neq 0, y \neq 0} \int d x_{r} d y_{r} \frac{\phi_{\pi}^{p}(x, y)}{x y} \delta(1-x-y)|x y|^{\epsilon}+\text { c.t. } \\
= & \left(\frac{\bar{n} \cdot p_{\pi}}{\mu_{-}}\right)^{2 \epsilon} \sum_{x \neq 0,1} \int d x_{r} \frac{\hat{\phi}_{\pi}^{p}(x)}{x(1-x)} \theta(x) \theta(1-x)|x(1-x)|^{\epsilon}+\text { c.t. } \\
= & \int_{0}^{1} d x\left\{\frac{\hat{\phi}_{\pi}^{p}(x)}{x(1-x)}-\frac{\hat{\phi}_{\pi}^{p}(0)}{x}-\frac{\hat{\phi}_{\pi}^{p}(1)}{1-x}\right\} \\
& -\left(\frac{\bar{n} \cdot p_{\pi}}{\mu_{-}}\right)^{2 \epsilon}\left\{\int_{1}^{\infty} d x \frac{\hat{\phi}_{\pi}^{p}(0) x^{\epsilon}(x-1)^{\epsilon}}{x}+\int_{-\infty}^{0} d x \frac{\hat{\phi}_{\pi}^{p}(1) x^{\epsilon}(1-x)^{\epsilon}}{1-x}\right\}+\text { c.t. } \\
= & \int_{0}^{1} d x\left\{\frac{\phi_{\pi}^{p}(x)}{x(1-x)}-\frac{\phi_{\pi}^{p}(0)}{x}-\frac{\phi_{\pi}^{p}(1)}{1-x}\right\}+\ln \left(\frac{\bar{n} \cdot p_{\pi}}{\mu_{-}}\right)\left[\phi_{\pi}^{p}(0)+\phi_{\pi}^{p}(1)\right]
\end{aligned}
$$

and

$$
\begin{aligned}
\int d x & d y \frac{\phi_{\pi}(x, y)}{\left(x^{2}\right)_{\varnothing}\left(y^{2}\right)_{\varnothing}} \delta(1-x-y) \\
= & \left(\frac{\bar{n} \cdot p_{\pi}}{\mu_{-}}\right)^{2 \epsilon} \sum_{x \neq 0,1} \int d x_{r} \frac{\hat{\phi}_{\pi}(x)}{x^{2}(1-x)^{2}} \theta(x) \theta(1-x)|x(1-x)|^{\epsilon}+\text { c.t. } \\
= & \int_{0}^{1} d x\left\{\frac{\hat{\phi}_{\pi}^{p}(x)}{x^{2}(1-x)^{2}}-\frac{\hat{\phi}_{\pi}^{\prime}(0)}{x}+\frac{\hat{\phi}_{\pi}^{\prime}(1)}{1-x}\right\} \\
& -\left(\frac{\bar{n} \cdot p_{\pi}}{\mu_{-}}\right)^{2 \epsilon}\left\{\int_{1}^{\infty} d x \frac{\hat{\phi}_{\pi}^{\prime}(0) x^{\epsilon}(x-1)^{\epsilon}}{x}-\int_{-\infty}^{0} d x \frac{\hat{\phi}_{\pi}^{\prime}(1) x^{\epsilon}(1-x)^{\epsilon}}{1-x}\right\}+\text { c.t. } \\
= & \int_{0}^{1} d x\left\{\frac{\phi_{\pi}(x)}{x^{2}(1-x)^{2}}-\frac{\phi_{\pi}^{\prime}(0)}{x}+\frac{\phi_{\pi}^{\prime}(1)}{1-x}\right\}+\ln \left(\frac{\bar{n} \cdot p_{\pi}}{\mu_{-}}\right)\left[\phi_{\pi}^{\prime}(0)-\phi_{\pi}^{\prime}(1)\right] .
\end{aligned}
$$

In the last line the sign for $\phi_{\pi}^{\prime}(1)$ appears because we differentiate with respect to $x$ rather than $1-x$. The notation + c.t. indicates the matrix element of the counterterm operators that cancel the $1 / \epsilon_{\mathrm{UV}}$ divergences leaving only the logarithm. The dependence of the results on $\mu$ was suppressed, and terms with counterterm coefficients, $C_{c t}^{[0]}\left(\mu_{-}\right)\left[\phi_{\pi}^{p}(0)+\phi_{\pi}^{p}(1)\right]$ and $C_{c t}^{[1]}\left(\mu_{-}\right)\left[\phi_{\pi}^{\prime}(0)-\phi_{\pi}^{\prime}(1)\right]$ should be added to these amplitudes 91 .

The final matrix elements in Eqs. (129, 133, 134, 135) have a linear $\ln \left(\mu_{-}\right)$dependence which comes from the action of the $\varnothing$-distribution. The coefficient of these logs is independent of the power taken for the $x^{\epsilon}$ type factors, though the analysis in section VIIB dictates that the $\epsilon$-power should be used. The $\ln \left(\mu_{-}\right)$dependence will be canceled order by order in $\alpha_{s}(\mu)$ by $\ln \left(\mu_{-}\right)$dependence in the perturbative kernel $J\left(\mu_{-}\right)$including the coefficients $C_{c t}\left(\mu_{-}\right)$of the counterterm operators. Just as in our scalar loop example in sections VII A 1 and VII B 1, when we consider the resulting factorization formula at the matching scale the $\mu_{+}$and $\mu_{-}$ dependence will cancel out between logs in the coefficient functions and those in the $\mu_{ \pm}$ dependent hadronic distributions, where $\mu^{2}=\mu_{+} \mu_{-}$. 


\section{The $\rho-\pi$ Form Factor at Large $Q^{2}$}

In this section we consider $\gamma^{*} \rho \rightarrow \pi$ as an example of a process with convolution integrals that appear to be divergent, but are tamed in our formulation of $\mathrm{SCET}_{\mathrm{II}}$ using the procedure in section VIIC. The QCD dynamics are described by the $\rho-\pi$ form factor

$$
\left\langle\pi^{+}\left(p^{\prime}\right)\left|\bar{q} \gamma^{\nu} q\right| \rho^{+}\left(p, \varepsilon^{\perp}\right)\right\rangle=i \epsilon^{\nu \alpha \beta \lambda} p_{\alpha} p_{\beta}^{\prime} \varepsilon_{\lambda}^{\perp} F_{\rho \pi}\left(q^{2}\right),
$$

with $\varepsilon^{\perp}$ the transverse rho polarization vector. At large $Q^{2}=-q^{2}$ the form factor $F_{\rho \pi}\left(q^{2}\right)$ was studied in detail in Ref. [23], and also was discussed in [92]. The pion form factor $F_{\pi \pi}\left(q^{2}\right)$ and leading proton form factor $F_{p p}\left(q^{2}\right)$ both scale as $1 / Q^{2}$ at large $Q^{2}$, however the form factor $F_{\rho \pi}\left(q^{2}\right)$ scales as $1 / Q^{4}$. This results from an additional $1 / Q$ suppression of the QCD matrix element shown in Eq. (136) relative to the pion and proton cases.

In SCET, the two LO currents $J_{j}^{(0)}$ that mediate any $\gamma^{*} M_{1} \rightarrow M_{2}$ transition with light quarks were derived in Ref. 66]. Using isospin and working in the Breit frame with incoming momentum transfer $q^{\mu}=Q \bar{n}^{\mu} / 2-Q n^{\mu} / 2$, the incoming partons are $n$-collinear and outgoing partons are $\bar{n}$-collinear. The LO matching of the QCD vector current onto SCET is

$$
\begin{aligned}
& J^{\nu} \rightarrow \frac{n^{\nu}+\bar{n}^{\nu}}{Q^{3}} \int d \omega_{j}\left[C_{1}\left(\mu, \omega_{j}\right) J_{1}^{(0)}\left(\mu, \omega_{j}\right)+C_{2}\left(\mu, \omega_{j}\right) J_{2}^{(0)}\left(\mu, \omega_{j}\right)\right], \\
& J_{j}^{(0)}=\left[\bar{\chi}_{n, \omega_{1}} \Gamma_{j} \chi_{n, \omega_{2}}\right]\left[\bar{\chi}_{\bar{n}, \omega_{3}} \Gamma_{j}^{\prime} \chi_{\bar{n}, \omega_{4}}\right] \text {, }
\end{aligned}
$$

and involves $n$-collinear isodoublet $\chi_{n}$ and $\bar{n}$-collinear isodoublet $\chi_{\bar{n}}$ fields in $\mathrm{SCET}_{\mathrm{II}}$. Since the $\rho$ and $\pi$ have opposite charge conjugation, the only relevant matrix from Ref. [66] for our two isotriplet mesons is

$$
\Gamma_{2} \otimes \Gamma_{2}^{\prime}=\frac{1}{4}\left[\left(Q_{u}+Q_{d}\right)\left(\tau^{a} \otimes \tau^{a}\right)\left[\not h \otimes \not h+\not h \gamma_{5} \otimes \not h \gamma^{5}\right] .\right.
$$

From Eq. (138) we see that there is no $\gamma_{\perp}$, so at this order the $\rho_{\perp}-\pi$ transition is forbidden. This is a reflection of the helicity structure of the factorization [23, 92, 92] and occurs despite the existence of matrix elements at leading power for the relevant hadronic states:

$$
\begin{aligned}
\left\langle 0\left|\bar{d}_{n,-y} \not h \gamma_{\perp}^{\mu} u_{n, x}\right| \rho_{n}^{+}\left(p, \varepsilon_{\perp}\right)\right\rangle & =f_{\rho}^{T} \bar{n} \cdot p \varepsilon_{\perp}^{\mu} \delta(1-x-y) \phi_{\rho_{\perp}}(\mu, x, y), \\
\left\langle\pi_{\bar{n}}^{+}\left(p^{\prime}\right)\left|\bar{u}_{\bar{n}, u} \not h \gamma_{5} d_{\bar{n},-v}\right| 0\right\rangle & =-i f_{\pi} n \cdot p^{\prime} \delta(1-u-v) \phi_{\pi}(\mu, u, v) .
\end{aligned}
$$

These twist-2 matrix elements will be useful below. The notation $u_{n, x}$ denotes a gauge invariant product of a Wilson line and quark with momentum fraction $x$ along the quark arrow, $u_{n, x}=\delta(x-\overline{\mathcal{P}} / \bar{n} \cdot p) W^{\dagger} \xi_{n}^{(u)}$. Similarly, $\bar{d}_{n,-y}=\bar{\xi}_{n}^{(d)} W \delta\left(y+\overline{\mathcal{P}}^{\dagger} / \bar{n} \cdot p\right)$ etc. Finally, the $\delta(1-x-y)$ factors on the r.h.s. of Eq. (139) comes from momentum conservation in the matrix element. When implementing the $\varnothing$-distribution we start with expressions containing $\delta$ functions, like those shown in Eq. (139). This is useful for cases where there are simultaneous zero-bin subtractions in variables appearing in the $\delta$-function. 
Once operators suppressed by $1 / Q$ are considered, the $\rho_{\perp}^{+} \rightarrow \pi^{+}$transition is allowed [23]. In $\mathrm{SCET}_{\mathrm{II}}$ the relevant operators can be constructed from products of the bilinear $\mathcal{O}\left(\eta^{3}\right)$ operators from Ref. 93.

$$
\begin{aligned}
P_{ \pm}(u, v) & =\left[\bar{u}_{\bar{n}} \frac{\not h}{2}\left(\mathcal{D}_{\bar{n}}^{\perp}\right)^{\dagger}\right]_{u} \frac{\gamma_{5}}{\mathcal{P}^{\dagger}} d_{\bar{n},-v} \pm \bar{u}_{\bar{n}, u} \frac{\gamma_{5}}{\mathcal{P}}\left[\left(\not \mathcal{D}_{\bar{n}}^{\perp}\right) \frac{\not h}{2} d_{\bar{n}}\right]_{-v}, \\
V_{1 \pm}^{\mu}(x, y) & =\left[\bar{d}_{n} \frac{\not h}{2}\left(\mathcal{D}_{\bar{n}}^{\perp}\right)^{\dagger}\right]_{-y} \frac{\gamma^{\mu}}{\overline{\mathcal{P}}^{\dagger}} u_{n, x} \pm \bar{d}_{n,-y} \frac{\gamma^{\mu}}{\overline{\mathcal{P}}}\left[\left(i \mathcal{D}_{\bar{n}}^{\perp}\right) \frac{\not h}{2} u_{n}\right]_{x}, \\
V_{2 \pm}^{\mu}(x, y) & =\left[\bar{d}_{n} \frac{\not h}{2}\left(i \mathcal{D}_{\bar{n}}^{\perp \mu}\right)^{\dagger}\right]_{-y} \frac{1}{\overline{\mathcal{P}}^{\dagger}} u_{n, x} \pm \bar{d}_{n,-y} \frac{1}{\overline{\mathcal{P}}}\left[\left(i \mathcal{D}_{\bar{n}}^{\perp \mu}\right) \frac{\not h}{2} d_{n}\right]_{x}, \\
T_{3}^{\mu \nu}(u, v, w) & =\bar{u}_{\bar{n}, u} \frac{\not h \gamma_{\perp}^{\mu}}{2}\left(i g \mathcal{B}_{\bar{n} \perp}^{\nu}\right)_{-w} d_{\bar{n},-v}, \\
V_{3}^{\mu}(x, y, z) & =\bar{d}_{n,-y} \frac{\not h}{2}\left(i g \mathcal{B}_{n \perp}^{\mu}\right)_{z} u_{n, x}, \\
A_{3}^{\mu}(x, y, z) & =\bar{d}_{n,-y} \frac{\not h \gamma_{5}}{2}\left(i g \mathcal{B}_{n \perp}^{\mu}\right)_{z} u_{n, x},
\end{aligned}
$$

where $\overline{\mathcal{P}}=\bar{n}_{\alpha} \mathcal{P}^{\alpha}, \mathcal{P}=n_{\alpha} \mathcal{P}^{\alpha}, i g \mathcal{B}_{n \perp}^{\mu}=\left[1 / \overline{\mathcal{P}} W_{n}^{\dagger}\left[i \bar{n} \cdot D_{n}, i D_{n \perp}^{\mu}\right] W_{n}\right], i \mathcal{D}_{n}^{\perp \mu}=\mathcal{P}_{\perp}^{\mu}+i g \mathcal{B}_{n \perp}^{\mu}$, and $\left(i g \mathcal{B}_{n \perp}^{\mu}\right)_{z}=\delta(z-\overline{\mathcal{P}} / n \cdot p) i g \mathcal{B}_{n \perp}^{\mu}$. As indicated, in this section we use a rescaling with respect to the momentum carried by the state in order to make the delta functions acting on the fields dimensionless. This rescaling hides the process independence of the operators, but makes the results simpler to present. Note that we have used slightly different notation for the operators than Ref. 93], with relations $P_{+}(u, v)=\mathcal{P}^{\mathrm{LPW}}(\vec{\omega}), P_{-}(u, v)=\tilde{\mathcal{P}}^{\mathrm{LPW}}(\vec{\omega})$, etc. We also included one less power of $1 / \overline{\mathcal{P}}$ in the three-body operators.

For the two-body operators we have matrix elements 93.

$$
\begin{aligned}
\left\langle\pi_{\bar{n}}^{+}\left|P_{+}(u, v)\right| 0\right\rangle & =-i f_{\pi} \mu_{\pi} \delta(1-u-v) \phi_{\pi}^{p}(\mu, u, v), \\
\left\langle\pi_{\bar{n}}^{+}\left|P_{-}(u, v)\right| 0\right\rangle & =\frac{-i f_{\pi} \mu_{\pi}}{6} \delta(1-u-v) \phi_{\pi}^{\prime \sigma}(\mu, u, v), \\
\left\langle 0\left|V_{1+}^{\mu}(x, y)\right| \rho_{n \perp}^{+}(\varepsilon)\right\rangle & =f_{\rho} m_{\rho} \varepsilon_{\perp}^{\mu} \delta(1-x-y) g_{\rho_{\perp}}^{(v)}(\mu, x, y), \\
\left\langle 0\left|V_{1-}^{\mu}(x, y)\right| \rho_{n \perp}^{+}(\varepsilon)\right\rangle & =\frac{f_{\rho} m_{\rho} \varepsilon_{\perp}^{\mu}}{4} \delta(1-x-y) g_{\rho_{\perp}}^{(a) \prime}(\mu, x, y) \\
& =\frac{f_{\rho} m_{\rho} \varepsilon_{\perp}^{\mu}}{2(y-x)} \delta(1-x-y) g_{\rho_{\perp}}^{(A)}(\mu, x, y),
\end{aligned}
$$

where $\mu_{\pi}=m_{\pi}^{2} /\left(m_{u}+m_{d}\right)$ and for later convenience we switch from the distribution $g_{\rho_{\perp}}^{(a) \prime}$ to a distribution $g_{\rho_{\perp}}^{(A)}$. Other matrix elements are related to these by simple operator relations in SCET. For the three-body operators the matrix elements are 93. 

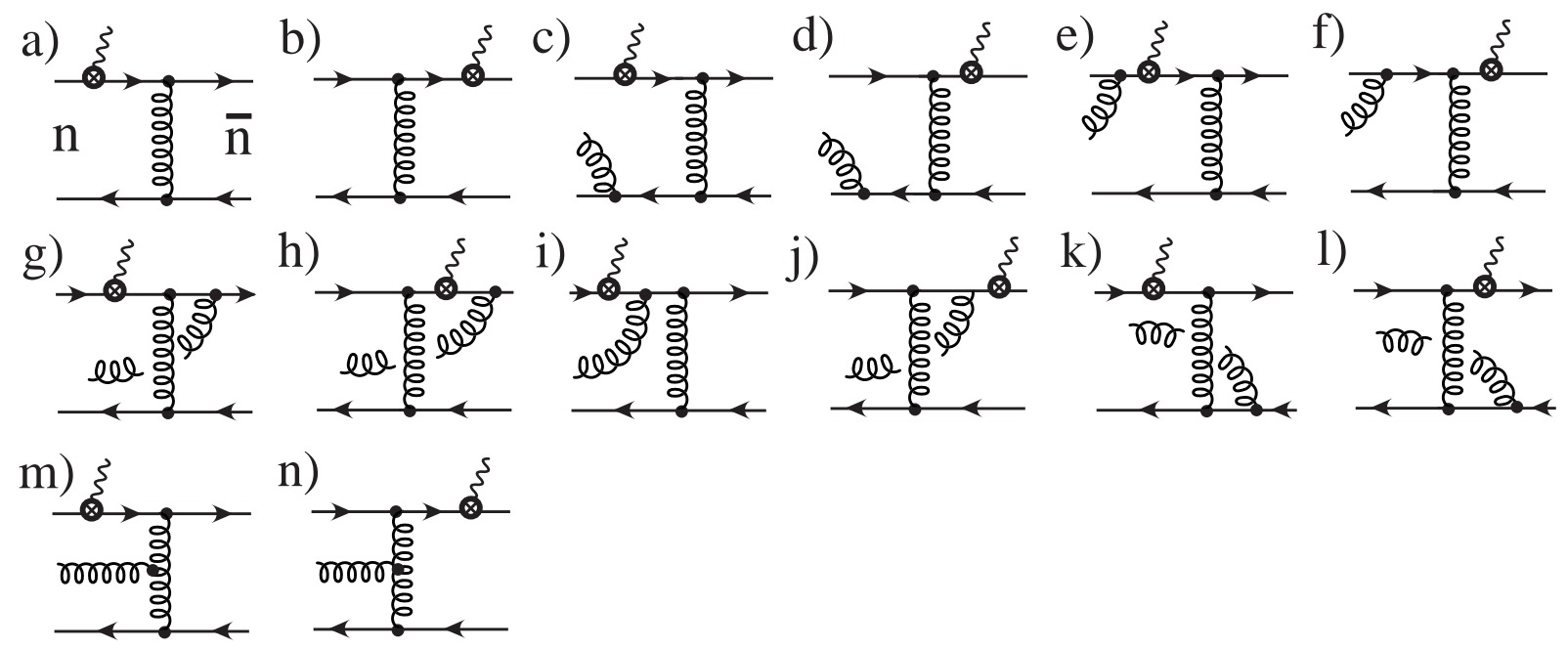

FIG. 16: Graphs for matching onto the $\rho-\pi$ electromagnetic form factor. To obtain the full set of diagrams one must add graphs with the gluon exiting to the right (the left-right mirror images of c) through $\mathrm{n}$ ) not flipping the direction of the arrows). Then to this entire set one must add the graphs with the current insertion on the other quark line (which can be obtained by charge conjugation).

$$
\begin{gathered}
\left\langle\pi_{\bar{n}}^{+}\left(p^{\prime}\right)\left|T_{3}^{\mu \nu}(u, v, w)\right| 0\right\rangle=n \cdot p^{\prime} f_{3 \pi} \epsilon_{\perp}^{\mu \nu} \frac{\delta(1-u-v-w)}{2 w} \phi_{3 \pi}(\mu, u, v, w), \\
\left\langle 0\left|V_{3}^{\mu}(x, y, z)\right| \rho_{n \perp}^{+}(p, \varepsilon)\right\rangle=-\bar{n} \cdot p f_{3 \rho}^{V} \varepsilon_{\perp}^{\mu} \frac{\delta(1-x-y-z)}{2 z} \phi_{3 \rho}^{V}(\mu, x, y, z), \\
\left\langle 0\left|A_{3}^{\mu}(x, y, z)\right| \rho_{n \perp}^{+}(p, \varepsilon)\right\rangle=i \bar{n} \cdot p f_{3 \rho}^{A} \epsilon_{\perp}^{\mu \nu} \varepsilon_{\nu}^{\perp} \frac{\delta(1-x-y-z)}{2 z} \phi_{3 \rho}^{A}(\mu, x, y, z),
\end{gathered}
$$

where $\epsilon_{\perp}^{\mu \nu}=\epsilon^{\mu \nu \alpha \beta} \bar{n}_{\alpha} n_{\beta} / 2$ (which switches sign under $n \leftrightarrow \bar{n}$ ). In comparing to Ref. 93] note that we took $\left(f_{V} m_{V} \mathcal{V}\right)^{\mathrm{LPW}}=-f_{\rho}^{3 V} \phi_{\rho}^{3 V}$ and $\left(f_{V} m_{V} \mathcal{A}\right)^{\mathrm{LPW}}=f_{3 \rho}^{A} \phi_{3 \rho}^{A}$ which agrees with the notation in Ref. [23]. To compare with the other notation in Ref. [23], note that $\left(\varphi_{\pi}^{A}\right)^{\mathrm{CZ}}=$ $\phi_{\pi} / 2,\left(\varphi_{\pi}^{p}\right)^{\mathrm{CZ}}=\phi_{\pi}^{p} / 2,\left(\varphi_{3 \pi}\right)^{\mathrm{CZ}}=\phi_{3 \pi},\left(\varphi_{\rho}^{T}\right)^{\mathrm{CZ}}=\phi_{\rho_{\perp}} / 2,\left(\varphi_{\rho}^{V, \perp}\right)^{\mathrm{CZ}}=g_{\rho_{\perp}}^{(v)} / 2,\left(\varphi_{\rho}^{A, \perp}\right)^{\mathrm{CZ}}=$ $(1-2 z) g_{\rho_{\perp}}^{(a) \prime}(z) / 4=g_{\rho_{\perp}}^{(A)}(z) / 2$.

To connect the operators in Eq. (140) to the process $\gamma^{*} \rho_{\perp}^{+} \rightarrow \pi^{+}$we must match the full theory diagrams shown in Fig. 16 onto $\mathrm{SCET}_{\mathrm{II}}$. The graphs in Fig. 16a-b must be expanded to NLO. The graphs in Fig. 16c-f can be obtained from graphs a) and b) by using the tree level relation between the QCD and $\mathrm{SCET}_{\mathrm{II}}$ fields in place of the lowest order spinor in the LO part of these diagrams:

$$
\psi=W\left[1+\frac{1}{\overline{\mathcal{P}}} i \not_{n \perp} \frac{\not h}{2}\right]\left(W^{\dagger} \xi_{n}\right)
$$

Graphs g) through n) require separate computations.

In matching these graphs onto SCET $_{\text {II }}$ we can make different assumptions for the scaling of the external lines. We work in the Breit frame and take the interpolating field for the 
incoming $\rho$ and outgoing $\pi$ to be built purely out of $n$ and $\bar{n}$ collinear fields respectively. The contribution that matches onto the operators in Eqs. (139,140) will have two $n$-collinear quark fields and two $\bar{n}$-collinear quark fields with or without an extra $n$ or $\bar{n}$ collinear gluon. In addition $\mathrm{SCET}_{\mathrm{II}}$ has graphs where one or more of the above fields simultaneously become soft. Although we can formulate operators with soft fields in $\mathrm{SCET}_{\mathrm{II}}$, they do not contribute to the $\rho_{\perp}-\pi$ form factor in this frame. The zero-bin subtractions ensures that we will not double count the region of momentum space that these other operators correctly describe. Following Ref. [35] we note that we do not need to consider interpolating fields for hadrons built out of mixed soft and collinear components. These interpolating fields do not have nonperturbative poles as discussed earlier. Furthermore, in the Breit frame, an interpolating field that is purely soft would only be needed for a different physical process than the one we are considering (and would correspondingly require different current operators).

To simplify the presentation we define

$$
\delta_{x y}=\delta(1-x-y), \quad \delta_{x y z}=\delta(1-x-y-z)
$$

Using the computation of the tree level graphs done in Ref. [23], but including the $\sum_{p^{ \pm} \neq 0}$ terms with $\varnothing$-distributions as described in the previous section, gives ${ }^{23}$

$$
\begin{aligned}
& F_{\rho \pi}\left(Q^{2}\right)=\frac{4 \pi \alpha_{s}(\mu)}{27 Q^{4}} \int d x \int d y \int d z \int d u \int d v \int d w\left\{4 f_{\rho}^{T} f_{\pi} \mu_{\pi} \frac{\delta_{x y} \delta_{u v} \phi_{\rho_{\perp}}(x, y) \phi_{\pi}^{p}(u, v)}{\left(y^{2}\right)_{\varnothing} v_{\varnothing}}\right. \\
& +f_{\rho}^{V} m_{\rho} f_{\pi} \delta_{x y} \delta_{u v}\left[\frac{g_{\rho_{\perp}}^{(v)}(x, y) \phi_{\pi}(u, v)}{x_{\varnothing} y_{\varnothing}\left(v^{2}\right)_{\varnothing}}+\frac{g_{\rho_{\perp}}^{(A)}(x, y) \phi_{\pi}(u, v)}{4 x_{\varnothing} y_{\varnothing}\left(u^{2}\right)_{\varnothing}\left(v^{2}\right)_{\varnothing}}\right]+\frac{f_{\rho}^{3 A} f_{\pi}}{4} \delta_{u v} \delta_{x y z} \phi_{\pi}(u, v) \phi_{3 \rho}(x, y, z) \\
& \times\left[\frac{8}{\left(\bar{y}^{2} x\right)_{\varnothing} v_{\varnothing}}+\frac{2}{(\bar{z} z y)_{\varnothing} v_{\varnothing}}-\frac{9}{\left(\bar{y}^{2} x\right)_{\varnothing}\left(v^{2}\right)_{\varnothing}}-\frac{1}{(\bar{z} z x)_{\varnothing}\left(v^{2}\right)_{\varnothing}}-\frac{1}{\left(z \bar{y}^{2}\right)_{\varnothing}\left(v^{2}\right)_{\varnothing}}\right] \\
& -f_{\rho}^{T} f_{3 \pi} \phi_{3 \pi}(u, v, w) \phi_{\rho_{\perp}}(x, y) \delta_{u v w} \delta_{x y}\left[\frac{9}{2\left(\bar{u}^{2} v\right)_{\varnothing}\left(y^{2}\right)_{\varnothing}}+\frac{1}{2\left(\bar{u}^{2} w\right)_{\varnothing}\left(y^{2}\right)_{\varnothing}}+\frac{1}{(\bar{u} v w)_{\varnothing} y_{\varnothing}}\right] \\
& + \text { D-terms }\},
\end{aligned}
$$

where $\phi_{3 \rho}=\phi_{\rho}^{3 A}-f_{\rho}^{3 V} / f_{\rho}^{3 A} \phi_{\rho}^{3 V}$. The "+ D-terms" factor indicates that at this order we must also include the renormalized coefficients $D_{i}\left(\mu_{+}, \mu_{-}\right)$just like the coefficient $C_{c t}^{[1]}\left(\mu_{-}\right)$ in Eq. (128). Here they multiply terms with $\phi_{\rho_{\perp}}^{\prime}(1), g_{\rho_{\perp}}^{(A)}(0), g_{\rho_{\perp}}^{(A)}(1), g_{\rho_{\perp}}^{(v)}(0), g_{\rho_{\perp}}^{(v)}(1), \phi_{\pi}^{\prime}(0)$, $\phi_{\pi}^{\prime}(1)$, and $\phi_{\pi}^{p}(1)$. The $\mu$-dependence of the distributions is suppressed for brevity. The range for the integrations are determined by the theta functions in the non-perturbative distributions, which have support from $[0,1]$ in their respective momentum fraction variables. Any variable denoted with a bar is one minus itself, $\bar{x}=1-x$, etc. For the three-body distributions we will have two convolution integrals left after using the $\delta$-functions, and the

${ }^{23}$ Note that we have not independently verified the calculations in Ref. [23]. 
$\varnothing$-distribution must in general be treated as two-dimensional. We indicated this in Eq. (146) by having the $\varnothing$ subscript act on the product of three-body momentum fractions. The action of $\varnothing$ in these cases can be determined by the same steps used in section VIIG. Due to the $\varnothing$-distributions the result in Eq. (146) is finite, independent of assumptions about the non-perturbative distribution functions.

It is possible to study the $\mu_{ \pm}$dependence of the result in Eq. (146). To do so one adopts some endpoint behavior for the distributions, and can make the action of the $\varnothing$ distributions from the tree level jet functions explicit. A common assumption for the scaling behavior of the above distribution functions near their endpoints is $\phi_{\pi}(x) \sim x \bar{x}, \phi_{\rho_{\perp}}(u) \sim u \bar{u}$, $g_{\rho_{\perp}}^{(v)}(x) \sim 1, g_{\rho_{\perp}}^{(a)}(x) \sim x \bar{x}$ (so $\left.g_{\rho_{\perp}}^{(A)}(x) \sim 1\right), \phi_{\pi}^{p}(u) \sim 1, \phi_{3 \rho}(x, y) \sim x y z^{2}, \phi_{3 \pi}(u, v) \sim u v w^{2}$. With this scaling behavior all integrals over three body distributions converge without zerobin subtractions, and we can evaluate the two-body $\varnothing$-distributions explicitly using the formulas worked out in section VII . For the rapidity logarithms in the distributions we then get $\ln \left(\bar{n} \cdot p_{\rho} / \mu_{-}\right)=\ln \left(Q / \mu_{-}\right)$and $\ln \left(n \cdot p_{\pi} / \mu_{+}\right)=\ln \left(Q / \mu_{+}\right)$. The $\mu_{+}$and $\mu_{-}$dependence in these logarithms is canceled by the $\mu_{ \pm}$dependence of the $D_{i}\left(\mu_{ \pm}\right)$Wilson coefficients.

In this section we showed that the $\mathrm{SCET}_{\mathrm{II}}$ zero-bin subtractions together with UV renormalization yield a finite answer for the $\rho-\pi$ form factor at large $Q^{2}$, given in Eq. (146). Due to the separation in rapidity, the result has additional dependence on $\ln (Q)$ beyond that in the hard scattering kernel. The appearance of these logarithms is controlled by the powers of momentum fractions in the hard scattering kernel and the endpoint behavior of the distributions. At the matching scale $\mu_{+} \gtrsim \Lambda_{\mathrm{QCD}}$ and $\mu_{-} \sim Q$ and there are no large logarithms. As we scale $\mu^{2}$ towards $\Lambda_{\mathrm{QCD}}^{2}$ keeping $\mu^{2}=\mu_{+} \mu_{-}$, large logarithms may be generated. The dependence on this large $\log$ is computable, up to its normalization which is fixed by non-perturbative parameters. These parameters are determined once we adopt a model for the light-cone distribution functions.

\section{E. Factorization of $\zeta^{B \pi}(E)$ appearing in $B \rightarrow \pi \ell \bar{\nu}$ and $B \rightarrow \pi \pi$}

In this section, we study the implications of our results for $B \rightarrow \pi \ell \bar{\nu}$ and the related process $B \rightarrow \pi \pi$. For small pion energy $E$, this process is dominated by the $B^{*}$ pole and can be studied in chiral perturbation theory [94, 95]. For $E \lesssim 1 \mathrm{GeV}$ this process is also amenable to HQET and lattice QCD simulations [96, 97]. For large $E$, namely $E^{2} \gg E \Lambda_{\mathrm{QCD}} \gg \Lambda_{\mathrm{QCD}}^{2}$, the process is expected to factor into the convolution of perturbative and non-perturbative contributions.

The exploration of factorization in QCD for the process $B \rightarrow \pi \ell \bar{\nu}$ has a rich history. In Ref. [25], exclusive QCD factorization techniques were applied to the $B \rightarrow \pi$ and $B \rightarrow$ $\pi \pi$ transitions to examine the hard scattering contributions. Endpoint divergences as in Eq. (22) were encountered. Ref. [98] argued that the soft endpoint contributions dominate,

and obey heavy quark symmetry relations. This was extended to a bigger class of large 
energy symmetry relations in Ref. [99] in the context of an EFT known as LEET [100] that was later shown to be inconsistent. Factorization of soft and collinear regions was studied in Ref. [24], including Sudakov suppression of soft contributions, and a result for the hard parts was obtained in terms of leading twist $B$ and $\pi$ distributions. $B \rightarrow \pi \ell \bar{\nu}$ decays were studied with light-cone sum rules in Refs. [101, 102, 103], and in Ref. [103, a definition of the soft and hard contributions was given using the so-called local duality approximation. The soft part dominated numerically. In Ref. [104], it was shown that terms with endpoint divergences could be absorbed into a soft form factor without spoiling the large energy symmetry relations, and $\alpha_{s}$ contributions which spoil the relations were evaluated. A dominant non-factorizable soft form factor is an important ingredient in the power counting used by BBNS in discussing factorization for $B \rightarrow \pi \pi$ [105. ${ }^{24}$ In formulating SCET in Ref. [2], the leading order low energy current operators for the soft $B \rightarrow \pi$ form factor were derived, and their hard Wilson coefficients were computed at $\mathcal{O}\left(\alpha_{s}\right)$. Ref. 108 studied $B \rightarrow \pi$ form factors with threshold and $k_{\perp}$ resummations, argued that soft contributions are Sudakov suppressed in this framework, pointed out the importance of the $p^{2} \sim m_{b} \Lambda_{\mathrm{QCD}}$ scale, and obtained a factorized result given by individual $B$ and $\pi$ wavefunctions with $k_{\perp}$ 's. The ability to completely factorize the amplitude into individual $B$ and $\pi$ distributions depending on $k_{\perp}$ is an important ingredient in the pQCD approach to $B \rightarrow \pi \pi$ [30].

More recently, $\mathrm{SCET}_{\mathrm{I}}$ and $\mathrm{SCET}_{\mathrm{II}}$ have been used to analyze $B \rightarrow \pi \ell \bar{\nu}$. Refs. [35, 109] pointed out that the interpolating field for the pion is purely collinear, and that the LO terms therefore necessarily involve time-ordered products of currents and subleading Lagrangians in $\mathrm{SCET}_{\mathrm{I}}$. Thus, in the complete set of operator contributions, leading and subleading currents both contribute to the LO form factor. The hard-scattering and soft form factor contributions can be defined as subsets of these time-ordered products with $J^{(1)}$ and $J^{(0)}$, and it was shown that the factorization of the hard-scattering contributions $J^{(1)}$ involve a hard function for the scale $m_{b}^{2}$, and also a distinct jet function for the scale $m_{b} \Lambda_{\mathrm{QCD}}$. In Refs. [75, 87], factorization in $\mathrm{SCET}_{\mathrm{II}}$ was further investigated, and it was argued that soft-collinear messenger contributions spoil the possibility of further factorization of the soft $B \rightarrow \pi$ form factor $J^{(0)}$ terms. In Ref. [78], the implications of endpoint singularities for factorization in SCET were investigated. It was shown that the hard-scattering contributions are finite to all orders in $\alpha_{s}$. It was also argued that endpoint singularities spoil possible factorization of the soft form factor terms below the scale $m_{b} \Lambda_{\mathrm{QCD}}$. In Ref. 80], the complete set of $\operatorname{SCET}_{\text {II }}$ operators for the soft $B \rightarrow \pi$ form factor contribution were determined, including three body operators. In Refs. [110] a factorization formula for $B \rightarrow \pi \pi$ was investigated in SCET and it was pointed out that the same universal jet function occurs as in the $B \rightarrow \pi \ell \bar{\nu}$ process (for a precursor see [111]).

The above results can be summarized by the following formula for the relevant $B \rightarrow \pi$

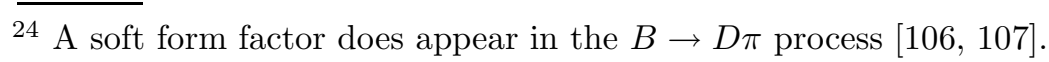


form factor, derived from a systematic expansion in $E \gg \Lambda_{\mathrm{QCD}}$ :

$$
\begin{aligned}
f_{+}(E) & =T^{(+)}(E) \zeta^{B \pi}(E)+\int_{0}^{1} d z C_{J}^{(+)}(z, E) \zeta_{J}^{B \pi}(z, E) \\
& =T^{(+)}(E) \zeta^{B \pi}(E)+N_{0} \int_{0}^{1} d z \int_{0}^{1} d x \int_{0}^{\infty} d k_{+} C_{J}^{(+)}(z, E) J\left(z, x, k_{+}, E\right) \phi_{\pi}(x) \phi_{B}^{+}\left(k_{+}\right) .
\end{aligned}
$$

Our notation follows Refs. [109, 110, so $N_{0}=f_{B} f_{\pi} m_{B} /\left(4 E^{2}\right)$. The hard functions $T^{(+)}$and $C_{J}^{(+)}$are perturbative at $\mu^{2} \sim m_{b}^{2}$, the jet function $J$ is perturbative at $\mu^{2} \sim E \Lambda_{\mathrm{QCD}}$, and $\phi_{\pi}$ and $\phi_{B}^{+}$are leading twist distributions, which are non-perturbative. Finally, $\zeta^{B \pi}(E)=$ $\left(m_{b}^{1 / 2} / E^{2}\right) \hat{\zeta}^{B \pi}(E)$ is the unfactorized soft form factor with $\hat{\zeta}^{B \pi}(E)$ containing dependence on the scales $E \Lambda_{\mathrm{QCD}}$ and $\Lambda_{\mathrm{QCD}}^{2}$. At lowest order in $\alpha_{s}\left(m_{b}\right)$ at the hard scale $T^{(+)}=1, C_{J}^{(+)}=1$, and $f_{+}(E)=\zeta^{B \pi}(E)+\zeta_{J}^{B \pi}(E)$, where $\zeta_{J}^{B \pi}(E)=\int d z \zeta_{J}^{B \pi}(z, E)$. If we also work to order $\alpha_{s}(\mu)$ at the intermediate jet scale, $\mu^{2} \sim E \Lambda_{\mathrm{QCD}}$, we can expand further, and Eq. (147) gives

$$
f_{+}(E)=\zeta^{B \pi}(E)+\frac{f_{B} f_{\pi} m_{B}}{4 E^{2}} \frac{4 \pi \alpha_{s}(\mu)}{9}\left(\frac{2 E}{m_{B}}+\frac{2 E}{m_{b}}-1\right) \int_{0}^{1} d x \frac{\phi_{\pi}(x)}{x} \int_{0}^{\infty} d k^{+} \frac{\phi_{B}\left(k^{+}\right)}{k^{+}} .
$$

In this result the $\zeta^{B \pi}(E)$ term is left unexpanded since its factorization at scales $\mu^{2} \lesssim E \Lambda_{\mathrm{QCD}}$ is (so far) unknown. In the remainder of this section we will use the zero-bin in $\mathrm{SCET}_{\text {II }}$ to demonstrate that $\zeta^{B \pi}(E)$ can be factorized further into products of twist-two and twist-three $\pi$ and $B$ distribution functions.

In $\mathrm{SCET}_{\mathrm{I}}$ the $\zeta^{B \pi}(E)$ term is defined by

$$
\begin{aligned}
\left\langle\pi_{n}\left(p_{\pi}^{-}\right)\left|T_{0}+T_{3}+T_{4}+T_{5}+T_{6}\right| B_{v}\right\rangle & =\bar{n} \cdot p_{\pi} C\left(p_{\pi}^{-}\right) \zeta^{B \pi}\left(p_{\pi}^{-}\right) \\
& =2 E C(E) \zeta^{B \pi}(E)
\end{aligned}
$$

The $T_{i}$ are time-ordered products of subleading Lagrangians with the leading scalar heavyto-light current $J^{(0)}=\sum_{p^{-}} C\left(p^{-}\right)\left(\bar{\xi}_{n} W\right)_{p^{-}}\left(Y^{\dagger} h_{v}\right)$. Their definitions are 109, 110

$$
\begin{array}{ll}
T_{0}=i \int d^{4} y T J^{(0)}(0) \mathcal{L}_{\xi q}^{(1)}(y), & T_{3}=i \int d^{4} y T J^{(0)}(0) \mathcal{L}_{\xi q}^{(2 b)}(y), \\
T_{4}=i \int d^{4} y T J^{(0)}(0) \mathcal{L}_{\xi q}^{(2 a)}(y), & T_{5}=i^{2} \int d^{4} y d^{4} z T J^{(0)}(0) \mathcal{L}_{\xi \xi}^{(1)}(y) \mathcal{L}_{\xi \xi}^{(1)}(z), \\
T_{6}=i^{2} \int d^{4} y d^{4} z T J^{(0)}(0) \mathcal{L}_{\xi q}^{(1)}(y) \mathcal{L}_{c g}^{(1)}(z), &
\end{array}
$$

and the presence of only $J^{(0)}$ guarantees that $\zeta^{B \pi}(E)$ satisfies the symmetry relations of Ref. [99]. The momentum conserving $\delta$-function collapse the matrix element of the $T_{i}$ 's into a simple product. The momentum $p_{\pi}^{-} \sim m_{b}$ is large, and $p_{\pi}^{-}=2 E$ up to small power suppressed $m_{\pi}$ dependent terms.

The tree level matching onto $\mathrm{SCET}_{\mathrm{II}}$ for $J^{(0)}$ currents was carried out in Ref. [80]. They 
found the position space operators

$$
\begin{aligned}
& O_{1}^{(P)}=g^{2}\left[\bar{\chi}(0) \frac{\not h \gamma_{5}}{2} \chi(s \bar{n})\right]\left[\overline{\mathcal{Q}}_{s}(t n) \frac{\not h h \gamma_{5}}{4} \mathcal{H}(0)\right], \\
& O_{2}^{(P)}=g^{2}\left[\bar{\chi}(0) \frac{\not h \gamma_{5}}{2} i \not_{\perp} \chi(s \bar{n})\right]\left[\overline{\mathcal{Q}}_{s}(t n) \frac{\not h \gamma_{5}}{2} \mathcal{H}(0)\right], \\
& O_{3}^{(P)}=g^{2}\left[\bar{\chi}(0) \frac{\not h \gamma_{5}}{2} \mathcal{A}_{c \perp}(r \bar{n}) \chi(s \bar{n})\right]\left[\overline{\mathcal{Q}}_{s}(t n) \frac{\not h \gamma_{5}}{2} \mathcal{H}(0)\right], \\
& O_{4}^{(P)}=g^{2}\left[\bar{\chi}(0) \frac{\not h \gamma_{5}}{2} \chi(s \bar{n})\right]\left[\overline{\mathcal{Q}}_{s}(t n) \mathcal{A}_{s \perp}(\text { un }) \frac{\not h \gamma_{5}}{2} \mathcal{H}(0)\right],
\end{aligned}
$$

where the notation is defined in Ref. [80]. The time-ordered product $T_{0}$ contributes to the matching onto operators with a gluon field strength. They also had a fifth operator, $\mathrm{O}_{5}$, involving a time-ordered product with soft-collinear messenger fields. In our setup the infrared regions associated to the messenger fields are covered by the soft and collinear fields so this fifth operator should not be included. With labeled fields the operators are

$$
\begin{aligned}
O_{1} & =\left[\left(\bar{\xi}_{n} W\right)_{u} \frac{\not h \gamma_{5}}{2}\left(W^{\dagger} \xi_{n}\right)_{-v}\right]\left[\left(\bar{q}_{s} S\right)_{-k_{1}} \frac{\not h h \gamma_{5}}{4}\left(S^{\dagger} h_{v}\right)_{k_{2}}\right], \\
O_{2} & =\left[\left(\bar{\xi}_{n} W\right)_{u} \frac{\not h \gamma_{5}}{2} \not{P}_{\perp}\left(W^{\dagger} \xi_{n}\right)_{-v}\right]\left[\left(\bar{q}_{s} S\right)_{-k_{1}} \frac{\not h \gamma_{5}}{2}\left(S^{\dagger} h_{v}\right)_{k_{2}}\right], \\
O_{3} & =\left[\left(\bar{\xi}_{n} W\right)_{u} \frac{\not h \gamma_{5}}{2}\left(i g \mathcal{B}_{n}^{\perp}\right)_{-w}\left(W^{\dagger} \xi_{n}\right)_{-v}\right]\left[\left(\bar{q}_{s} S\right)_{-k_{1}} \frac{\not h \gamma_{5}}{2}\left(S^{\dagger} h_{v}\right)_{k_{2}}\right], \\
O_{4} & =\left[\left(\bar{\xi}_{n} W\right)_{u} \frac{\not h \gamma_{5}}{2}\left(W^{\dagger} \xi_{n}\right)_{-v}\right]\left[\left(\bar{q}_{s} S\right)_{-k_{1}}\left(i g \mathcal{B}_{s}^{\perp}\right)_{k_{3}} \frac{\not h \gamma_{5}}{2}\left(S^{\dagger} h_{v}\right)_{k_{2}}\right] .
\end{aligned}
$$

Tree level matching gives the Wilson coefficients 80]

$$
\begin{aligned}
& J_{1}^{\pi}=-\frac{4 \pi \alpha_{s}(\mu)}{9 E^{2}}\left[\frac{1}{v^{2} k_{1}}+\frac{1}{v k_{1}}\right] \\
& J_{2}^{\pi}=-\frac{4 \pi \alpha_{s}(\mu)}{9 E^{2}} \frac{1}{u v^{2} k_{1}^{2}}, \\
& J_{3}^{\pi}=\frac{\pi \alpha_{s}(\mu)}{2 E^{2}}\left[\frac{w}{(v+w)^{2} v k_{1}^{2}}-\frac{7}{9(v+w)^{2} k_{1}^{2}}-\frac{8}{9 v(u+w) k_{1}^{2}}\right], \\
& J_{4}^{\pi}=\frac{\pi \alpha_{s}(\mu)}{2 E^{2}}\left[\frac{k_{3}}{v^{2}\left(k_{1}+k_{3}\right)^{2} k_{1}}-\frac{8 k_{3}}{9 v\left(k_{1}+k_{3}\right)^{2} k_{1}}+\frac{1}{9 v^{2}\left(k_{1}+k_{3}\right)^{2}}\right] .
\end{aligned}
$$

In Eq. (152), the $\left(i g \mathcal{B}_{n}^{\perp}\right)$ was defined below Eq. (140), and $i g \mathcal{B}_{s \perp}^{\mu}=\left[1 / \mathcal{P} S^{\dagger}\left[i n \cdot D_{s}, i D_{s \perp}^{\mu}\right] S\right]$ with $S\left[n \cdot A_{s}\right]$ soft Wilson lines. As in the previous section, we rescaled the delta functions acting on the collinear fields by $\bar{n} \cdot p_{\pi}$ so that the subscripts involve the momentum fractions $u, v, w$, and the delta functions set either $u+v=1$ or $u+v+w=1$. For the soft fields we left the delta functions dimension-full, e.g. $\left(\bar{q}_{s} S\right)_{-k_{1}}=\left(\bar{q}_{s} S\right) \delta\left(k_{1}+\mathcal{P}^{\dagger}\right)$. Note that the vacuum to pion matrix element of the operators $O_{2}^{(P)}$ and $O_{2}$ will contribute. ${ }^{25}$

\footnotetext{
${ }^{25}$ We thank B. Lange for pointing this out.
} 
The factorization in rapidity in SCET $_{\mathrm{II}}$ discussed in section VII indicates that the jet function matching which determines Eq. (153) will give factors of $\alpha_{s}(\mu)$ that are perturbative at the jet scale $\mu^{2} \sim E \Lambda_{\mathrm{QCD}}$, similar to the $\alpha_{s}(\mu)$ in Eq. (148). Therefore we included the $\alpha_{s}(\mu)$ in the $J_{i}^{\pi}$ coefficients, rather than grouping a $g^{2}$ with the operators in Eq. (152). To evaluate the $\langle\pi|\cdots| B\rangle$ matrix elements of the $O_{1,2,3,4}$, we will need Eqs. (139) and (143), the pion matrix element

$$
\begin{aligned}
& \left\langle\pi_{n}(p)\left|\left(\bar{\xi}_{n} W\right)_{u} \frac{\not h \gamma_{5}}{2} \not_{\perp}\left(W^{\dagger} \xi_{n}\right)_{-v}\right| 0\right\rangle \\
& =\left\langle\pi_{n}(p)\left|\frac{(-u n \cdot p)}{2}\left(P_{+}+P_{-}\right)(u, v)-i \epsilon_{\mu \nu}^{\perp} \int d w T_{3}^{\mu \nu}(u-w, v, w)\right| 0\right\rangle \\
& \quad=\frac{i}{2} \bar{n} \cdot p f_{\pi} \mu_{\pi} \delta_{u v} u\left[\phi_{\pi}^{p}(\mu, u, v)+\frac{1}{6} \phi_{\pi}^{\prime \sigma}(\mu, u, v)\right]+i \bar{n} \cdot p f_{3 \pi} \int d w \delta_{u v} \frac{\phi_{3 \pi}(u-w, v, w)}{w}
\end{aligned}
$$

and the $B$ matrix elements

$$
\begin{aligned}
\left\langle 0\left|\left(\bar{q}_{s} S\right)_{-k 1} \frac{\not h h \gamma_{5}}{4}\left(S^{\dagger} h_{v}\right)_{k 2}\right| B\right\rangle & =-i \frac{f_{B} m_{B}}{2} \delta\left(k_{1}+k_{2}-\bar{\Lambda}\right) \phi_{B}^{-}\left(k_{1}, k_{2}\right), \\
\left\langle 0\left|\left(\bar{q}_{s} S\right)_{-k 1} \frac{\not h \gamma_{5}}{2}\left(S^{\dagger} h_{v}\right)_{k 2}\right| B\right\rangle & =i \frac{f_{B} m_{B}}{2} \delta\left(k_{1}+k_{2}-\bar{\Lambda}\right) \phi_{B}^{+}\left(k_{1}, k_{2}\right), \\
\left\langle 0\left|\left(\bar{q}_{s} S\right)_{-k 1}\left(i g B_{s}^{\perp}\right)_{k_{3}} \frac{\not h \gamma_{5}}{2}\left(S^{\dagger} h_{v}\right)_{k 2}\right| B\right\rangle & =i f_{3 B} m_{B} \delta\left(k_{1}+k_{2}+k_{3}-\bar{\Lambda}\right) \phi_{3 B}\left(k_{1}, k_{2}, k_{3}\right) .
\end{aligned}
$$

Here $f_{B} \sim m_{b}^{-1 / 2}$ can be taken to be the decay constant in the heavy quark limit, and our definition for $\phi_{B}^{ \pm}$is that of Ref. [112], while $\phi_{3 B}$ has been studied in Ref. [113] and is proportional to $\tilde{\Psi}_{A}-\tilde{\Psi}_{V}$ from Ref. 114. The momentum conserving delta functions involve the HQET mass of the $B$-state, $\bar{\Lambda}=\lim _{m_{b} \rightarrow \infty}\left(m_{B}-m_{b}\right)$. For the lowest order factorization formula for the soft form factor $\zeta^{B \pi}(E)$, we find

$$
\begin{aligned}
& \zeta^{B \pi}(E)=\frac{f_{\pi} f_{B} m_{B}}{4 E^{2}} \pi \alpha_{s}(\mu) \int_{0}^{1} d u d v d w \int d k_{1} d k_{2}\left\{\frac{4}{9} \delta_{k_{1} k_{2}} \delta_{u v} \frac{(1+v) \phi_{\pi}(u, v)}{\left(v^{2}\right)_{\varnothing}} \frac{\phi_{B}^{-}\left(k_{1}, k_{2}\right)}{\left(k_{1}\right)_{\varnothing}}\right. \\
& +\frac{4 \mu_{\pi}}{9} \delta_{k_{1} k_{2}} \delta_{u v} \frac{\left(\phi_{\pi}^{p}+\frac{1}{6} \phi_{\pi}^{\prime \sigma}\right)(u, v)}{\left(v^{2}\right)_{\varnothing}} \frac{\phi_{B}^{+}\left(k_{1}, k_{2}\right)}{\left(k_{1}^{2}\right)_{\varnothing}}+\frac{f_{3 B}}{f_{B}} \int d k_{3} \delta_{k_{1} k_{2} k_{3}} \delta_{u v}\left[\frac{\phi_{\pi}(u, v)}{\left(v^{2}\right)_{\varnothing}}\right. \\
& \left.\quad \times \phi_{3 B}\left(k_{1}, k_{2}, k_{3}\right) \frac{9 k_{3}+k_{1}}{9\left[\left(k_{1}+k_{3}\right)^{2} k_{1}\right]_{\varnothing}}-\frac{\phi_{\pi}(u, v)}{v_{\varnothing}} \frac{8 k_{3} \phi_{3 B}\left(k_{1}, k_{2}, k_{3}\right)}{9\left[\left(k_{1}+k_{3}\right)^{2} k_{1}\right]_{\varnothing}}\right] \\
& +\frac{f_{3 \pi}}{f_{\pi}} \delta_{k_{1} k_{2}} \delta_{u v w}\left[\frac{\phi_{3 \pi}(u, v, w)}{\left[(v+w)^{2} v\right]_{\varnothing}}-\frac{7 \phi_{3 \pi}(u, v, w)}{9\left[w(v+w)^{2}\right]_{\varnothing}}+\frac{8 \bar{v} \phi_{3 \pi}(u, v, w)}{9\left[v^{2} w(u+w)\right]_{\varnothing}}\right] \frac{\phi_{B}^{+}\left(k_{1}, k_{2}\right)}{\left(k_{1}^{2}\right)_{\varnothing}} \\
& + \text { D-terms }\} .
\end{aligned}
$$

Here $\bar{v}=1-v$, the $k_{i}$-limits are $-\infty<k_{2}<\bar{\Lambda}, 0<k_{1,3}<\infty$ and

$$
\begin{aligned}
\delta_{k_{1} k_{2}} & =\delta\left(\bar{\Lambda}-k_{1}-k_{2}\right), & \delta_{k_{1} k_{2} k_{3}} & =\delta\left(\bar{\Lambda}-k_{1}-k_{2}-k_{3}\right), \\
\delta_{u v} & =\delta(1-u-v), & \delta_{u v w} & =\delta(1-u-v-w) .
\end{aligned}
$$


The "+ D-terms" factor in Eq. (156) indicates that at this order we must also include the renormalized coefficients $D_{i}\left(\mu_{+}, \mu_{-}\right)$for operators like the ones in Eq. (128). In Eq. (156), we have obtained a finite factorization formula for the soft $B \rightarrow \pi$ form factor. The structure of this contribution is very similar to the hard-scattering term in Eq. (148), except for the necessity of the zero-bin subtractions.

To study the $\mu_{ \pm}$dependence in Eq. (156), we can adopt the standard endpoint behavior for the distributions. For simplicity, we can adopt a behavior for $\phi_{3 B}\left(k_{1}, k_{2}, k_{3}\right)$ so that its tree-level integrals also all converge without applying the zero-bin and renormalization. This leaves $\phi_{\pi}(u) \sim u \bar{u}, \phi_{B}^{+}\left(k_{1}\right) \sim k_{1}, \phi_{B}^{-}\left(k_{1}\right) \sim 1$, and $\phi_{3 \pi}(u, v, w) \sim u v w^{2}$. The zero-bin is then required for $\phi_{\pi}(u, v) /\left(v^{2}\right)_{\varnothing}$ given in Eq. (129) and for $\phi_{3 \pi}(u, v, w) /\left[v^{2} w(u+w)\right]_{\varnothing}$ which generate $\ln \left(\mu_{-} / p_{\pi}^{-}\right)=\ln \left(\mu_{-} / 2 E\right)$ terms, and for $\phi_{B}^{+}\left(k_{1}, k_{2}\right) /\left(k_{1}^{2}\right)_{\varnothing}$ and $\phi_{B}^{-}\left(k_{1}, k_{2}\right) /\left(k_{1}\right)_{\varnothing}$. Following the usual procedure, we find that these last two terms each generate a $\ln \left[\mu_{+} /(n \cdot v \bar{\Lambda})\right]$

At higher orders in $\alpha_{s}$, we anticipate that the factorization formula will take the form

$$
\begin{aligned}
\zeta^{B \pi} & =N_{0} \int d u \int d k_{1} \hat{J}_{1}\left(u, k_{1}, E, \mu, \mu^{ \pm}\right) \phi_{\pi}\left(u, \mu, \mu_{-}\right) \phi_{B}^{-}\left(k_{1}, \mu, \mu_{+}\right) \\
& +N_{0} \int d u \int d k_{1} \hat{J}_{2}\left(u, k_{1}, E, \mu, \mu^{ \pm}\right)\left(\phi_{\pi}^{p}+\frac{1}{6} \phi_{\pi}^{\prime \sigma}\right)\left(u, \mu, \mu_{-}\right) \phi_{B}^{+}\left(k_{1}, \mu, \mu_{+}\right) \\
& +N_{0} \int d u d v \int d k_{1} \hat{J}_{3}\left(u, v, k_{1}, E, \mu, \mu^{ \pm}\right) \phi_{3 \pi}\left(u, v, \mu, \mu_{-}\right) \phi_{B}^{+}\left(k_{1}, \mu, \mu_{+}\right) \\
& +N_{0} \int d u \int d k_{1} d k_{2} \hat{J}_{4}\left(u, k_{1}, k_{2}, E, \mu, \mu^{ \pm}\right) \phi_{\pi}\left(u, \mu, \mu_{-}\right) \phi_{3 B}\left(k_{1}, k_{2}, \mu, \mu_{+}\right) .
\end{aligned}
$$

where we have used the $\delta_{k_{1} k_{2}}$ etc. functions to reduce the number of integrations, and we note that the zero-bin subtractions are present on the remaining variables. Here the $\mu_{ \pm}$ dependence in the $\phi_{\pi}$ 's and $\phi_{B}$ 's is a short hand for the $\ln \left(\mu_{-}\right)$and $\ln \left(\mu_{+}\right)$terms that are generated from the $\varnothing$-distribution depending on the structure of the $\hat{J}_{i}$ 's and the endpoint behavior of the distributions. The completeness of the mixed soft-collinear basis of operators $O_{1,2,3,4}$ found in Ref. [80] should guarantee that it is the non-perturbative functions shown in Eq. (158) which will show up at any order in $\alpha_{s}$ in the matching. ${ }^{26}$ One-loop corrections to $T^{(+)}, C_{J}^{(+)}$, and to $J$ are known [2, 21, 115, 116]. One-loop corrections for the $\hat{J}_{i}$ can be computed following the method outlined here.

Since the factorization formula for $B \rightarrow \pi \pi$ involves the same $\zeta^{B \pi}$ and $\zeta_{J}^{B \pi}$ functions,

$$
\begin{aligned}
A(\bar{B} \rightarrow \pi \pi) & =\lambda_{c}^{(f)} A_{c \bar{c}}^{M_{1} M_{2}}+\frac{G_{F} m_{B}^{2}}{\sqrt{2}}\left\{f_{\pi} \zeta^{B \pi} \int_{0}^{1} d u\left[T_{1 \zeta}(u)+T_{2 \zeta}(u)\right] \phi^{\pi}(u)\right. \\
& \left.+f_{\pi} \int_{0}^{1} d u \int_{0}^{1} d z\left[T_{1 J}(u, z)+T_{2 J}(u, z)\right] \phi^{\pi}(u) \zeta_{J}^{B \pi}(z)\right\},
\end{aligned}
$$

the results in this section immediately carry over to that process. In Eq. (159) $\zeta_{(J)}^{B \pi}=$ $\zeta_{(J)}^{B \pi}\left(E=m_{b} / 2\right)$ and we quoted the result from Ref. [110]. Our result in Eq. (156) lends

${ }^{26}$ The results in Eq. $(77,78)$ of Ref. [93], allow for the elimination of $\phi_{\pi}^{p}$ and $\phi_{\pi}^{\prime \sigma}$ in terms of $\phi_{3 \pi}$ if so desired. 
support to the power counting for $B \rightarrow \pi \pi$ used in Ref. [110], where $\zeta^{B \pi}$ and $\zeta_{J}^{B \pi}$ were treated as being parametrically and numerically similar in size. It also indicates that the entire non-leptonic tree amplitude for $B \rightarrow \pi \pi$ can likely be written in terms of individual $B$ and $\pi$ distribution functions. This differs from the BBNS [105, 117 type factorization, where the soft-form factor is taken to be non-perturbative. Our results appear to indicate

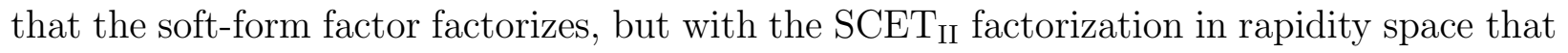
is different from the standard type of factorization formula. A factorization of the form factor in terms of individual $B$ and $\pi$ objects is similar to the result found in the pQCD analysis in Ref. [29, 30], however we do not find $k_{\perp}$ dependent functions. A more detailed study of Eqs. (156) will be reported on in a future publication.

\section{CONCLUSION}

This paper discusses how to formulate effective field theories with multiple fields for the same physical particle, where each field represents a region of momentum space with a different power counting. This allowed for a tiling of all the infrared momentum regions of the theory. The technique was applied to a non-relativistic field theory (NRQCD) and to field theories for energetic particles, including $\mathrm{SCET}_{\mathrm{I}}$ with collinear and ultrasoft quarks and gluons, and $\mathrm{SCET}_{\mathrm{II}}$ with soft and collinear quarks and gluons. Effective theories with multiple fields for the same particle can be formulated using fields with labels that distinguish the different momentum modes. In converting the sum over momentum labels into an integral, one must be careful with the zero-bin, where the momentum label is zero, since this corresponds to a different degree of freedom. Here the proper treatment of the zero-bin was investigated in detail. Sums which do not include the zero-bin are converted to integrals over all space with a zero-bin subtraction. The zero-bin subtraction removes the support of the integrand in the infrared region of momentum space where overlap between different modes could occur, and thus avoids double-counting between different modes in the effective theory.

The zero-bin subtractions give a definition of NRQCD and SCET independent of the choice of UV and IR regulators, allowing the use of regulators other than dimensional regularization if desired. They also solve a number of puzzles encountered in the literature on collinear factorization in QCD and non-relativistic field theories, which are associated with unphysical pinch and endpoint singularities, as well as puzzles in the NRQCD and SCET literature with UV and IR divergences that occur at intermediate length scales.

In NRQCD, the zero-bin subtractions eliminate pinch singularities in box-type graphs by properly distinguishing between potential and soft heavy fermions. This result applies to any non-relativistic effective theory (for example, it also simplifies the resolution of the puzzle discussed in Ref. [118] involving soft pions interacting with nucleons). Zero-bin subtractions also distinguish between soft and ultrasoft gluons in NRQCD. In graphs with soft loops, 
they convert infrared divergences into ultraviolet divergences. Converting an infrared into an ultraviolet divergences in the soft sector corresponds to a rearrangement of degrees of freedom that was known to be necessary in NRQCD, and had been implemented by hand as the "pull-up" procedure [14, 15]. Here a proper treatment of the zero-bin enabled us to derive the pull-up mechanism directly from the effective Lagrangian.

In $\mathrm{SCET}_{\mathrm{I}}$ zero-bin subtractions occur in collinear loops and avoid the overlap with the ultrasoft momentum region. These subtractions remove infrared divergences and induce new divergences in the ultraviolet. Previous results in the SCET literature used anomalous dimensions computed by including infrared divergences in collinear graphs as though they were ultraviolet divergences. This gives (by hand) the correct result for the anomalous dimensions. The zero-bin subtractions allows the computation of anomalous dimensions in the effective theory to be carried out by the standard renormalization procedure, in terms of the counterterms used to cancel ultraviolet divergences, and also ensures that IR divergences of the full theory are properly reproduced independent of the regulator choice. In inclusive decays at large energy, the zero-bin subtraction applies to both virtual loop integrals as well as phase space integrals for real emission as described in section VID. We believe that zerobin subtractions will play a role in the SCET matching calculations for parton showering carried out in Ref. [119, and perhaps also to the subtractions carried out in Ref. [120. They are likely to have implications for the singularities encountered with $k_{\perp}$-distributions with light-like Wilson lines which were discussed in Refs. [31, 32].

In exclusive decays, the zero-bin subtraction gives factorized decay rates and crosssections with finite convolution integrals. Convolution integrals of the perturbatively calculable kernel with hadron wavefunctions are sometimes naively divergent at the endpoints, as is the case for the $\rho-\pi$ form factor, and in factorization of the soft form factor in $B \rightarrow \pi \ell \nu$ decays. The zero-bin subtraction avoids double counting between soft and collinear modes in $\mathrm{SCET}_{\mathrm{II}}$, and this converts the unphysical infrared divergence in convolution integrals into an ultraviolet divergence. These ultraviolet divergences are canceled by operator renormalization. The final convolution integral is finite, determined by a distribution we called $\varnothing$. The $\varnothing$-distribution is a plus-type distribution augmented by additional non-analytic $\ln (E)$ dependence induced by the renormalization. Independent of the choice for the UV and IR regulators, our formulation of $\mathrm{SCET}_{\mathrm{II}}$ has only soft and collinear degrees of freedom and does not have soft-collinear messenger modes [75] as explained in section VIIA 1 and Appendix B. ${ }^{27}$

Thus one gets finite unambiguous formulas for amplitudes in $\mathrm{SCET}_{\mathrm{II}}$, free of endpoint singularities in the convolution integrals. In cases where there would have been a divergent convolution we get a separation of modes in rapidity space with variables $\mu_{ \pm}$. This preserves

27 In Refs. 50, 71, 121 SCET was reformulated in position space to avoid the sums over label momenta. To formulate the zero-bin subtractions in position space one can take the Fourier transform of Eq. (17). 
the power counting and naive factorization in the kinematic variables that would be present if the convolutions were finite. To illustrate this, we derived finite amplitudes for the $\rho-\pi$ form factor at large $Q^{2}$, and for the soft form factor function $\zeta^{B \pi}\left(E_{\pi}\right)$ that appears in $B \rightarrow \pi \ell \bar{\nu}$ and $B \rightarrow \pi \pi$ decays. Without a proper treatment of the zero-bin the convolutions in these factorization formulas would suffer from endpoint singularities. Applying the technique to the soft form factor for $B \rightarrow \pi \pi$ decays allowed us to derive an amplitude for non-leptonic decays that is entirely in terms of $B$ and $\pi$ distribution functions at lowest order.

In previous literature, the field theories, $\mathrm{SCET}_{\mathrm{I}}$ and $\mathrm{SCET}_{\mathrm{II}}$, have provided an algebraic means of deriving factorization formulas at any order in $\Lambda_{\mathrm{QCD}} / Q$, but not the means to guarantee that the manipulations would result in finite factorization formulas. Our work suggests that this will indeed be the case, so that the power expansion of observables can be carried out to any desired order without encountering singularities in convolution integrals. It still remains to carry out the full derivation of an SCET $_{\text {II }}$ factorization formula and explore the renormalization group properties of the resulting amplitudes - tasks which we leave for future work. The zero-bin procedure provides the freedom to tile the infrared of an EFT with suitable degrees of freedom, and makes the connection between the choice of degrees of freedom and the power counting expansion clear. Exploiting this, we converted the question of finding a complete set of degrees of freedom for an EFT at any order in the expansion, to a question that is easier to answer physically, that of identifying the relevant operators that occur at leading order for an observable, and give a proper formulation of the power counting.

\section{Acknowledgments}

We give a special thanks to A. Hoang for discussions on this topic over the years. We also acknowledge conversations with C. Bauer, D. Pirjol, and I. Rothstein. We thank B. Lange, Z. Ligeti, and S. Mantry for comments on the manuscript. This work was supported in part by the U.S. Department of Energy (DOE) under grant DE-FG03-97ER40546, the cooperative research agreement DF-FC02-94ER40818, and by the Office of Nuclear Science. AM would like to thank the Alexander von Humboldt foundation and the Max-Planck-Institut für Physik (Werner-Heisenberg-Institut) for support. I.S. was also supported in part by the DOE Outstanding Junior Investigator program and the Sloan foundation.

\section{APPENDIX A: RAPIDITY CUTOFF LOOP INTEGRALS IN SCET II}

In this section we give some details on the calculation of the integrals required for the SCET II diagrams in section VIIA. This calculations uses dimensional regularization for IR divergences, and cutoffs on a Wick rotated rapidity variable, $\zeta_{k}^{\prime}$, to regulate rapidity effects in the UV as in Eq. (94). The integrals we wish to 
compute are

$$
\begin{aligned}
I_{s} & =\int \frac{\mathrm{d}^{D} k}{(2 \pi)^{D}} \frac{1}{-p^{-} k^{+}+i 0^{+}} \frac{1}{k^{+} k^{-}-\mathbf{k}_{\perp}^{2}+i 0^{+}} \frac{1}{k^{+} k^{-}-k^{-} \ell^{+}-\mathbf{k}_{\perp}^{2}+i 0^{+}}, \\
I_{c} & =\int \frac{\mathrm{d}^{D} k}{(2 \pi)^{D}} \frac{1}{-k^{-} \ell^{+}+i 0^{+}} \frac{1}{k^{+} k^{-}-\mathbf{k}_{\perp}^{2}+i 0^{+}} \frac{1}{k^{+} k^{-}-k^{+} p^{-}-\mathbf{k}_{\perp}^{2}+i 0^{+}} .
\end{aligned}
$$

with $\ell^{+}>0$ and $p^{-}>0$. We use variables $\left\{k^{+}, \zeta_{k}\right\}$ for the soft integral with Wick rotation $\zeta_{k}=i \zeta_{k}^{\prime}$, and $\left\{k^{-}, \zeta_{k}\right\}$ for the collinear integral with $\zeta_{k}=-i \zeta_{k}^{\prime}$, as discussed in section VII A. It is easy to verify for $I_{s}$ and $I_{c}$ that these Wick rotations about the origin do not encounter any poles.

\section{$\underline{\text { Soft Integral in } \mathrm{SCET}_{\mathrm{II}}}$}

Consider the soft integral in Eq. (A1) and perform the $k_{\perp}$ integral:

$$
\begin{aligned}
I_{s} & =\int_{0}^{1} \mathrm{~d} x \int \frac{\mathrm{d}^{D} k}{(2 \pi)^{D}} \frac{1}{-p^{-} k^{+}+i 0^{+}} \frac{1}{\left[\mathbf{k}_{\perp}^{2}+k^{-} \ell^{+} x-k^{+} k^{-}-i 0^{+}\right]^{2}} \\
& =\frac{\Gamma(1+\epsilon)}{8 \pi} \int_{0}^{1} \mathrm{~d} x \int \frac{\mathrm{d} k^{+} \mathrm{d} k^{-}}{(2 \pi)^{2}} \frac{\left[k^{-} \ell^{+} x-k^{+} k^{-}-i 0^{+}\right]^{-1-\epsilon}}{-p^{-} k^{+}+i 0^{+}}
\end{aligned}
$$

Let $k^{-}=\zeta k^{+}$, and note that $d k^{-}=\left|k^{+}\right| d \zeta$ once we integrate $-\infty<k^{+}<\infty$ and $-\infty<\zeta<\infty$. Thus

$$
I_{s}=\frac{\Gamma(1+\epsilon)}{8 \pi p^{-}} \int_{0}^{1} \mathrm{~d} x \int \frac{\mathrm{d} k^{+} \mathrm{d} \zeta}{(2 \pi)^{2}} \frac{\left|k^{+}\right|\left[k^{+} \ell^{+} \zeta x-\left(k^{+}\right)^{2} \zeta-i 0^{+}\right]^{-1-\epsilon}}{-k^{+}+i 0^{+}}
$$

Rescale by a positive constant $k^{+}=\left(\ell^{+} x\right) k^{\prime+}$ and for simplicity rename $k^{+}=k^{+}$. Then rotate $\zeta=i \zeta^{\prime}$, so

$$
\begin{aligned}
I_{s} & =\frac{\Gamma(1+\epsilon)}{8 \pi p^{-}} \int_{0}^{1} \mathrm{~d} x\left(\ell^{+} x\right)^{-1-2 \epsilon} \int \frac{\mathrm{d} k^{+} \mathrm{d} \zeta}{(2 \pi)^{2}} \frac{\left|k^{+}\right|\left[\zeta k^{+}\left(1-k^{+}\right)-i 0^{+}\right]^{-1-\epsilon}}{-k^{+}+i 0^{+}} \\
& =\frac{-\Gamma(1+\epsilon)\left(\ell^{+}\right)^{-2 \epsilon}}{16 \pi \epsilon\left(p^{-} \ell^{+}\right)(2 \pi)^{2}} \int \mathrm{d} k^{+}\left(i \mathrm{~d} \zeta^{\prime}\right) \frac{\left|k^{+}\right|\left[i \zeta^{\prime} k^{+}\left(1-k^{+}\right)-i 0^{+}\right]^{-1-\epsilon}}{-k^{+}+i 0^{+}} .
\end{aligned}
$$

We divide the $k^{+}$integral into $k^{+}>1,0<k^{+}<1$, and $k^{+}<0$. This gives

$$
\begin{aligned}
J\left(\zeta^{\prime}\right) \equiv & \int_{-\infty}^{\infty} \mathrm{d} k^{+} \frac{\left|k^{+}\right|\left[i \zeta^{\prime} k^{+}\left(1-k^{+}\right)\right]^{-1-\epsilon}}{-k^{+}+i 0^{+}} \\
= & -\left(i \zeta^{\prime}\right)^{-1-\epsilon} \int_{0}^{1} \mathrm{~d} k^{+}\left[k^{+}\left(1-k^{+}\right)\right]^{-1-\epsilon}-\left(-i \zeta^{\prime}\right)^{-1-\epsilon} \int_{1}^{\infty} \mathrm{d} k^{+}\left[k^{+}\left(k^{+}-1\right)\right]^{-1-\epsilon} \\
& +\left(-i \zeta^{\prime}\right)^{-1-\epsilon} \int_{-\infty}^{0} \mathrm{~d} k^{+}\left[\left(-k^{+}\right)\left(1-k^{+}\right)\right]^{-1-\epsilon} \\
= & -\left(i \zeta^{\prime}\right)^{-1-\epsilon} \int_{0}^{1} \mathrm{~d} k^{+}\left[k^{+}\left(1-k^{+}\right)\right]^{-1-\epsilon} \\
= & -\left(i \zeta^{\prime}\right)^{-1-\epsilon} \frac{[\Gamma(-\epsilon)]^{2}}{\Gamma(-2 \epsilon)}=\left(i \zeta^{\prime}\right)^{-1-\epsilon}\left[\frac{2}{\epsilon}-\frac{\pi^{2} \epsilon}{3}-4 \zeta_{3} \epsilon^{2}+\ldots\right] .
\end{aligned}
$$

In the second equality note that the change of variables $k^{+} \rightarrow 1-k^{+}$for $k^{+}<0$ causes the second and third terms to cancel. Putting the pieces back together and multiplying by the $\mu^{2 \epsilon}$ this gives

$$
\begin{aligned}
I_{s} & =\frac{-\Gamma(1+\epsilon)}{64 \pi^{3} \epsilon\left(p^{-} \ell^{+}\right)} \frac{\left(\ell^{+}\right)^{-2 \epsilon}}{(\mu)^{-2 \epsilon}}\left[\frac{2}{\epsilon}-\frac{\pi^{2} \epsilon}{3}-4 \zeta_{3} \epsilon^{2}+\ldots\right] i \int_{\zeta_{\min }^{\prime}}^{\zeta_{\max }^{\prime}} d \zeta^{\prime}\left(i \zeta^{\prime}\right)^{-1-\epsilon} \\
& =\frac{\Gamma(1+\epsilon)}{64 \pi^{3} \epsilon^{2}\left(p^{-} \ell^{+}\right)} \frac{\left(\ell^{+}\right)^{-2 \epsilon}}{(\mu)^{-2 \epsilon}}\left[\frac{2}{\epsilon}-\frac{\pi^{2} \epsilon}{3}-4 \zeta_{3} \epsilon^{2}+\ldots\right]\left[\left(i \zeta_{\max }^{\prime}\right)^{-\epsilon}-\left(i \zeta_{\min }^{\prime}\right)^{-\epsilon}\right] .
\end{aligned}
$$


Now set $\zeta_{\min }^{\prime}=-a^{2}$ and $\zeta_{\max }^{\prime}=a^{2}$ and note that $( \pm i)^{-\epsilon}=\exp (\mp i \pi \epsilon / 2)$, so that we have

$$
\begin{aligned}
I_{s} & =\left[\frac{1}{64 \pi^{3}\left(p^{-} \ell^{+}\right)}\right] \frac{\Gamma(1+\epsilon)}{\epsilon} \frac{\left[e^{-i \pi \epsilon / 2}-e^{i \pi \epsilon / 2}\right]}{\epsilon}\left[\frac{2}{\epsilon}-\frac{\pi^{2} \epsilon}{3}-4 \zeta_{3} \epsilon^{2}+\ldots\right] \frac{\left(a \ell^{+}\right)^{-2 \epsilon}}{(\mu)^{-2 \epsilon}} \\
& =\left[\frac{-i \Gamma(1+\epsilon)}{32 \pi^{2}\left(p^{-} \ell^{+}\right)}\right] \frac{1}{\epsilon^{2}}\left[1-\frac{5 \pi^{2} \epsilon^{2}}{24}+\ldots\right] \frac{\left(a \ell^{+}\right)^{-2 \epsilon}}{(\mu)^{-2 \epsilon}} \\
& =\left[\frac{-i}{16 \pi^{2}\left(p^{-} \ell^{+}\right)}\right]\left[\frac{1}{2 \epsilon^{2}}-\frac{1}{\epsilon} \ln \left(\frac{a \ell^{+}}{\mu}\right)+\ln ^{2}\left(\frac{a \ell^{+}}{\mu}\right)-\frac{\pi^{2}}{16}+\ldots\right] .
\end{aligned}
$$

(In the last line when expanding in $\epsilon$ we have multiplied by the $\exp \left(\epsilon \gamma_{E}\right)$ factor to put $\mu$ into the $\overline{\mathrm{MS}}$ scheme, while the necessary $(4 \pi)^{-\epsilon}$ factor was removed already in Eq. (A2).) Eq. (A7) is the result quoted for the soft computation in Eq. (98) in the text.

Collinear integral in SCET $_{I I}$

Lets repeat the computation in the previous section for the collinear integral in Eq. (97). The integral is

$$
\begin{aligned}
I_{c} & =\int_{0}^{1} \mathrm{~d} x \int \frac{\mathrm{d}^{D} k}{(2 \pi)^{D}} \frac{1}{-k^{-} \ell^{+}+i 0^{+}} \frac{1}{\left[\mathbf{k}_{\perp}^{2}+p^{-} k^{+} x-k^{+} k^{-}-i 0^{+}\right]^{2}} \\
& =\frac{\Gamma(1+\epsilon)}{8 \pi} \int_{0}^{1} \mathrm{~d} x \int \frac{\mathrm{d} k^{+} \mathrm{d} k^{-}}{(2 \pi)^{2}} \frac{\left[k^{+} p^{-} x-k^{+} k^{-}-i 0^{+}\right]^{-1-\epsilon}}{-k^{-} \ell^{+}+i 0^{+}}
\end{aligned}
$$

Let $k^{+}=k^{-} / \zeta$ with $d k^{+}=\left|k^{-}\right| d \zeta / \zeta^{2}$ and $-\infty<\zeta<\infty$, so

$$
I_{c}=\frac{\Gamma(1+\epsilon)}{8 \pi \ell^{+}} \int_{0}^{1} \mathrm{~d} x \int \frac{\mathrm{d} k^{+} \mathrm{d} \zeta}{(2 \pi)^{2}} \frac{\left|k^{-}\right|\left[k^{-}\left(p^{-} x-k^{-}\right) / \zeta-i 0^{+}\right]^{-1-\epsilon}}{\zeta^{2}\left(-k^{-}+i 0^{+}\right)}
$$

Rescale by a positive constant $k^{+}=\left(p^{-} x\right) k^{\prime+}$ (for simplicity renaming $k^{\prime+} \rightarrow k^{+}$), and then rotate $\zeta=-i \zeta^{\prime}$,

$$
\begin{aligned}
I_{c} & =\frac{\Gamma(1+\epsilon)}{8 \pi \ell^{+}} \int_{0}^{1} \mathrm{~d} x\left(p^{-} x\right)^{-1-2 \epsilon} \int \frac{\mathrm{d} k^{-} \mathrm{d} \zeta}{(2 \pi)^{2}} \frac{\left|k^{-}\right|\left[k^{-}\left(1-k^{-}\right) / \zeta-i 0^{+}\right]^{-1-\epsilon}}{\zeta^{2}\left(-k^{-}+i 0^{+}\right)} \\
& =\frac{i \Gamma(1+\epsilon)\left(p^{-}\right)^{-2 \epsilon}}{64 \pi^{3} \epsilon\left(p^{-} \ell^{+}\right)} \int d \zeta^{\prime} \int_{-\infty}^{\infty} \mathrm{d} k^{-} \frac{\left|k^{-}\right|\left[i k^{-}\left(1-k^{-}\right) / \zeta^{\prime}-i 0^{+}\right]^{-1-\epsilon}}{\zeta^{\prime 2}\left(-k^{-}+i 0^{+}\right)} \\
& =\frac{\Gamma(1+\epsilon)\left(p^{-}\right)^{-2 \epsilon}}{64 \pi^{3} \epsilon\left(p^{-} \ell^{+}\right)} i \int_{\zeta_{\min }^{\prime}}^{\zeta_{\max }^{\prime}} \frac{d \zeta^{\prime}}{\zeta^{\prime 2}} J\left(1 / \zeta^{\prime}\right)
\end{aligned}
$$

where in the last line we noted that the $k^{-}$integral is identical $J\left(1 / \zeta^{\prime}\right)$ defined via Eq. (A5). Multiplying by $\mu^{2 \epsilon}$ this gives

$$
\begin{aligned}
I_{c} & =\frac{\Gamma(1+\epsilon)}{64 \pi^{3} \epsilon\left(p^{-} \ell^{+}\right)} \frac{\left(p^{-}\right)^{-2 \epsilon}}{(\mu)^{-2 \epsilon}}\left[\frac{2}{\epsilon}-\frac{\pi^{2} \epsilon}{3}-4 \zeta_{3} \epsilon^{2}+\ldots\right] i \int_{\zeta_{\min }^{\prime}}^{\zeta_{\max }^{\prime}} \frac{d \zeta^{\prime}}{\zeta^{\prime 2}}\left(\frac{i}{\zeta^{\prime}}\right)^{-1-\epsilon} \\
& =\frac{\Gamma(1+\epsilon)}{64 \pi^{3} \epsilon^{2}\left(p^{-} \ell^{+}\right)} \frac{\left(p^{-}\right)^{-2 \epsilon}}{(\mu)^{-2 \epsilon}}\left[\frac{2}{\epsilon}-\frac{\pi^{2} \epsilon}{3}-4 \zeta_{3} \epsilon^{2}+\ldots\right]\left[\left(\frac{i}{\zeta_{\max }^{\prime}}\right)^{-\epsilon}-\left(\frac{i}{\zeta_{\min }^{\prime}}\right)^{-\epsilon}\right] .
\end{aligned}
$$

Now we add the contributions from the regions $\zeta^{\prime} \in\left[a^{2}, \infty\right]$ and $\zeta^{\prime} \in\left[-\infty,-a^{2}\right]$ to give

$$
\begin{aligned}
I_{c} & =\left[\frac{1}{64 \pi^{3}\left(p^{-} \ell^{+}\right)}\right] \frac{\Gamma(1+\epsilon)}{\epsilon} \frac{\left[-e^{-i \pi \epsilon / 2}+e^{i \pi \epsilon / 2}\right]}{\epsilon}\left[\frac{2}{\epsilon}-\frac{\pi^{2} \epsilon}{6}-2 \zeta_{3} \epsilon^{2}+\ldots\right] \frac{\left(p^{-} / a\right)^{-2 \epsilon}}{(\mu)^{-2 \epsilon}} \\
& =\left[\frac{-i \Gamma(1+\epsilon)}{32 \pi^{2}\left(p^{-} \ell^{+}\right)}\right] \frac{1}{\epsilon^{2}}\left[1-\frac{5 \pi^{2} \epsilon^{2}}{24}+\ldots\right] \frac{\left(p^{-} / a\right)^{-2 \epsilon}}{(\mu)^{-2 \epsilon}} \\
& =\left[\frac{-i}{16 \pi^{2}\left(p^{-} \ell^{+}\right)}\right]\left[\frac{1}{2 \epsilon^{2}}-\frac{1}{\epsilon} \ln \left(\frac{p^{-}}{a \mu}\right)+\ln ^{2}\left(\frac{p^{-}}{a \mu}\right)-\frac{\pi^{2}}{16}+\ldots\right] .
\end{aligned}
$$

This is the collinear integral result quoted in Eq. (99). A simple way to get this answer is to note that the original collinear integral is identical to the soft integral with the replacements $k^{+} \leftrightarrow k^{-}, p^{-} \leftrightarrow \ell^{+}$, and $a \rightarrow 1 / a$. The answers in Eqs. A7) and (A12) agree with this. 


\section{APPENDIX B: SCET ${ }_{I I}$ LOOPS IN DIM. REG. WITH DIFFERENT IR REGU- LATORS}

In this appendix we repeat the matching computation done in section VII B 1 of a scalar loop integral in SCET $_{\text {II }}$. We use dimensional regularization, but modify the treatment of the IR regulator. The structure of the full theory and effective theory diagrams changes, but again the IR divergences are properly reproduced and the same contribution to the matching coefficient is obtained. The calculation is done for two classes of IR regulators: i) taking three different IR masses, $m_{1}, m_{2}$, and $m_{3}$ rather than just the single mass used in section VII B 1, and ii) with $m_{1} \neq 0, m_{2}=m_{3}=0$, and external momenta offshell, $\ell^{2} \neq 0$ and $p^{2} \neq 0$. Finally, in a part iii) we discuss subtleties related to the $m_{1}=0$ limit of these two cases. For simplicity we leave off the diagram prefactor $i e g^{2} G /\left(p^{-} \ell^{+}\right)$and just quote results for the integrals in this appendix. In all cases $p^{-}>0$ and $\ell^{+}>0$, and $\left(p^{-} \ell^{+}\right)$is the perturbative scale.

\section{i) Three IR Masses, $\mathrm{m}_{1}, \mathrm{~m}_{2}, \mathrm{~m}_{3}$}

The full theory loop is the generalization of Eq. VII B 1) with three IR masses,

$$
\begin{aligned}
I_{\text {full }}^{\text {scalar }} & =\int \frac{d^{D} k}{(2 \pi)^{D}} \frac{1}{\left[(k-\ell)^{2}-m_{2}^{2}+i 0^{+}\right]\left[k^{2}-m_{1}^{2}+i 0^{+}\right]\left[(k-p)^{2}-m_{3}^{2}+i 0^{+}\right]} \\
& =\frac{-i}{16 \pi^{2}\left(p^{-} \ell^{+}\right)}\left[\frac{1}{2} \ln ^{2}\left(\frac{m_{1}^{2}}{p^{-} \ell^{+}}\right)+\operatorname{Li}_{2}\left(1-\frac{m_{2}^{2}}{m_{1}^{2}}\right)+\operatorname{Li}_{2}\left(1-\frac{m_{3}^{2}}{m_{1}^{2}}\right)\right] .
\end{aligned}
$$

In Eq. (B1) factors of the IR regulators, $m_{1}^{2}, m_{2}^{2}$, and $m_{3}^{2}$ appear in all propagators, external momenta are taken onshell, $p^{2}=\ell^{2}=0$, and we have expanded in $m_{i}^{2} /\left(p^{-} \ell^{+}\right)$. The result is valid as long as $\left(p^{-} \ell^{+}\right) \gg m_{i}^{2}$, $\left(p^{-} \ell^{+}\right) m_{1}^{2} \gg m_{2}^{2} m_{3}^{2}$, and can not be used for the case $m_{1}=0$ since it blows up. The result which is valid for $m_{1} \rightarrow 0$ and also reproduces Eq. (B1) is given below in Eq. (B22). The $m_{1}=0$ result is in Eq. (B24).

The LO SCET II currents for dimensional regularization are given in Eq. (105). Using the $m_{1,2,3}$ IR regulators for the scalar and collinear loops in Figs. 15b,c we have

$$
\begin{aligned}
I_{\mathrm{soft}}^{\text {scalar }} & =\sum_{k^{+} \neq 0} \int \frac{d^{D} k_{r}}{(2 \pi)^{D}} \frac{\mu^{2 \epsilon}}{\left[k^{2}-\ell^{+} k^{-}-m_{2}^{2}+i 0^{+}\right]\left[k^{2}-m_{1}^{2}+i 0^{+}\right]\left[-p^{-} k^{+}+i 0^{+}\right]} \frac{\left|k^{+}\right|{ }^{\epsilon}\left|k^{+}-\ell^{+}\right|^{\epsilon}}{\mu_{+}^{2 \epsilon}}, \\
I_{\mathrm{cn}}^{\text {scalar }} & =\sum_{k^{-} \neq 0} \int \frac{d^{D} k_{r}^{\prime}}{(2 \pi)^{D}} \frac{\mu^{2 \epsilon}}{\left[-\ell^{+} k^{-}+i 0^{+}\right]\left[k^{2}-m_{1}^{2}+i 0^{+}\right]\left[k^{2}-p^{-} k^{+}-m_{3}^{2}+i 0^{+}\right]} \frac{\left|k^{-}\right|{ }^{\epsilon}\left|k^{-}-p^{-}\right|^{\epsilon}}{\mu_{-}^{2 \epsilon}} .
\end{aligned}
$$

Note that we keep $m_{2}^{2}$ and $m_{3}^{2}$ only in the propagators that are allowed to become small by the power counting in $\mathrm{SCET}_{\mathrm{II}}$. Eq. (17) tells us that we have zero-bin subtractions for the soft and collinear diagrams which avoid the IR singularities from the $\left[-p^{-} k^{+}\right]$and $\left[-\ell^{+} k^{-}\right]$propagators. The naive integrals and subtraction integrals are

$$
\begin{aligned}
\tilde{I}_{\text {soft }}^{\text {scalar }} & =\int \frac{d^{D} k}{(2 \pi)^{D}} \frac{\mu^{2 \epsilon}}{\left[k^{2}-\ell^{+} k^{-}-m_{2}^{2}+i 0^{+}\right]\left[k^{2}-m_{1}^{2}+i 0^{+}\right]\left[-p^{-} k^{+}+i 0^{+}\right]} \frac{\left|k^{+}\right|{ }^{\epsilon}\left|k^{+}-\ell^{+}\right|^{\epsilon}}{\mu_{+}^{2 \epsilon}}, \\
I_{\text {Osoft }}^{\text {scalar }} & =\int \frac{d^{D} k}{(2 \pi)^{D}} \frac{\mu^{2 \epsilon}}{\left[-\ell^{+} k^{-}+i 0^{+}\right]\left[k^{2}-m_{1}^{2}+i 0^{+}\right]\left[-p^{-} k^{+}+i 0^{+}\right]} \frac{\left|k^{+}\right|{ }^{\epsilon}\left|k^{+}-\ell^{+}\right|^{\epsilon}}{\mu_{+}^{2 \epsilon}}, \\
\tilde{I}_{\mathrm{cn}}^{\text {scalar }} & =\int \frac{d^{D} k}{(2 \pi)^{D}} \frac{\mu^{2 \epsilon}}{\left[-\ell^{+} k^{-}+i 0^{+}\right]\left[k^{2}-m_{1}^{2}+i 0^{+}\right]\left[k^{2}-p^{-} k^{+}-m_{3}^{2}+i 0^{+}\right]} \frac{\left|k^{-}\right|{ }^{\epsilon}\left|k^{-}-p^{-}\right|^{\epsilon}}{\mu_{-}^{2 \epsilon}}, \\
I_{0 \mathrm{cn}}^{\text {scalar }} & =\int \frac{d^{D} k}{(2 \pi)^{D}} \frac{\mu^{2 \epsilon}}{\left[-\ell^{+} k^{-}+i 0^{+}\right]\left[k^{2}-m_{1}^{2}+i 0^{+}\right]\left[-k^{-} k^{+}+i 0^{+}\right]} \frac{\left|k^{-}\right|^{\epsilon}}{\mu_{-}^{2 \epsilon}}
\end{aligned}
$$


To determine the form of the subtraction integrals, we considered the collinear limit of the soft loop momentum in $\tilde{I}_{\text {soft }}^{\text {scalar }}$, and the soft limit of the collinear loop momentum in $\tilde{I}_{\text {cn }}^{\text {scalar }}$. Note that the $m_{2}^{2}$ and $m_{3}^{2}$ dependence is dropped in the subtraction integrals because in these limits $\ell^{+} k^{-} \gg m_{2}^{2}$ and $p^{-} k^{+} \gg m_{3}^{2}$. The UV rapidity regulator factors, $|\cdots|^{\epsilon}$ are not affected by the subtractions (despite the way we are organizing the computation here, one really has an integrand defined with subtractions and then multiplies it by these factors). From Eq. (17) the differences $\tilde{I}_{\text {soft }}^{\text {scalar }}-I_{0 \text { soft }}^{\text {scalar }}$ and $\tilde{I}_{\text {cn }}^{\text {scalar }}-I_{0 \mathrm{cn}}^{\text {scalar }}$ will give the result for $I_{\text {soft }}^{\text {scalar }}$ and $I_{\mathrm{cn}}^{\text {scalar }}$ respectively.

For the soft graph we do the $k^{-}$integral by contours. Due to the pole structure this restricts the $k^{+}$ integration to the region $0<k^{+}<\ell^{+}$. The $k_{\perp}$ integral is then done. For the soft subtraction integral we follow the same procedure which this time leaves the integration region $0<k^{+}<\infty$. We find

$$
\begin{aligned}
\tilde{I}_{\text {soft }}^{\text {scalar }} & =\frac{-i \Gamma(\epsilon) \mu^{2 \epsilon}}{16 \pi^{2}\left(p^{-} \ell^{+}\right)} \int_{0}^{\ell^{+}} \frac{d k^{+}}{k^{+}}\left[m_{1}^{2}\left(1-\frac{k^{+}}{\ell^{+}}\right)+m_{2}^{2} \frac{k^{+}}{\ell^{+}}\right]^{-\epsilon}\left|\frac{k^{+}\left(k^{+}-\ell^{+}\right)}{\mu_{+}{ }^{2}}\right|^{\epsilon}, \\
I_{0 \text { soft }}^{\text {scalar }} & =\frac{-i \Gamma(\epsilon) \mu^{2 \epsilon}}{16 \pi^{2}\left(p^{-} \ell^{+}\right)} \int_{0}^{\infty} \frac{d k^{+}}{k^{+}}\left[m_{1}^{2}\right]^{-\epsilon}\left|\frac{k^{+}\left(k^{+}-\ell^{+}\right)}{\mu_{+}{ }^{2}}\right|^{\epsilon} .
\end{aligned}
$$

The double counting with the collinear integral comes from the $k^{+} \rightarrow 0$ part of the integral, but the divergence from this limit exactly cancels in $I_{\text {soft }}^{\text {scalar }}=\tilde{I}_{\text {soft }}^{\text {scalar }}-I_{0 \text { soft }}^{\text {scalar }}$ as long as $m_{1} \neq 0$. Computing the integrals we find

$$
\begin{aligned}
I_{\mathrm{soft}}^{\mathrm{scalar}}= & \frac{-i \Gamma(\epsilon)}{16 \pi^{2}\left(p^{-} \ell^{+}\right)}\left(\frac{\ell^{+}}{\mu_{+}}\right)^{2 \epsilon}\left\{\int_{0}^{1} d x \frac{|1-x|^{\epsilon}|x|^{\epsilon}}{x}\left[\frac{m_{1}^{2}(1-x)+m_{2}^{2} x}{\mu^{2}}\right]^{-\epsilon}-\int_{0}^{\infty} d x \frac{|1-x|^{\epsilon}|x|^{\epsilon}}{x}\left[\frac{m_{1}^{2}}{\mu^{2}}\right]^{-\epsilon}\right\} \\
= & \frac{-i \Gamma(\epsilon)}{16 \pi^{2}\left(p^{-} \ell^{+}\right)}\left(\frac{\mu^{2}}{m_{1}^{2}}\right)^{\epsilon}\left(\frac{\ell^{+}}{\mu_{+}}\right)^{2 \epsilon}\left\{\frac{\Gamma(\epsilon) \Gamma(1+\epsilon)}{\Gamma(1+2 \epsilon)}{ }^{2} F_{1}\left(\epsilon, \epsilon, 1+2 \epsilon, \frac{m_{1}^{2}-m_{2}^{2}}{m_{1}^{2}}\right)-\frac{\Gamma(\epsilon) \Gamma(1+\epsilon)}{\Gamma(1+2 \epsilon)}-\frac{\Gamma(1+\epsilon) \Gamma(-2 \epsilon)}{\Gamma(1-\epsilon)}\right\} \\
= & \frac{-i}{16 \pi^{2}\left(p^{-} \ell^{+}\right)}\left[\frac{1}{2 \epsilon_{\mathrm{UV}}^{2}}+\frac{1}{\epsilon_{\mathrm{UV}}} \ln \left(\frac{\ell^{+}}{\mu_{+}}\right)-\frac{1}{2 \epsilon_{\mathrm{UV}}} \ln \left(\frac{m_{1}^{2}}{\mu^{2}}\right)+\ln ^{2}\left(\frac{\ell^{+}}{\mu_{+}}\right)+\frac{5 \pi^{2}}{24}\right. \\
& \left.+\frac{1}{4} \ln ^{2}\left(\frac{m_{1}^{2}}{\mu^{2}}\right)-\ln \left(\frac{m_{1}^{2}}{\mu^{2}}\right) \ln \left(\frac{\ell^{+}}{\mu_{+}}\right)+\operatorname{Li}_{2}\left(1-\frac{m_{2}^{2}}{m_{1}^{2}}\right)\right]
\end{aligned}
$$

For the collinear integrals we do the contour integration in $k^{+}$which restricts the remaining integration region in $k^{-}$. For the naive and subtraction integrals we find

$$
\begin{aligned}
\tilde{I}_{\mathrm{cn}}^{\text {scalar }} & =\frac{-i \Gamma(\epsilon) \mu^{2 \epsilon}}{16 \pi^{2}\left(p^{-} \ell^{+}\right)} \int_{0}^{p^{-}} \frac{d k^{-}}{k^{-}}\left[m_{1}^{2}\left(1-\frac{k^{-}}{p^{-}}\right)+m_{3}^{2} \frac{k^{-}}{p^{-}}\right]^{-\epsilon}\left|\frac{k^{-}\left(k^{-}-p^{-}\right)}{\mu_{-}{ }^{2}}\right|^{\epsilon}, \\
I_{0 \mathrm{cn}}^{\text {scalar }} & =\frac{-i \Gamma(\epsilon) \mu^{2 \epsilon}}{16 \pi^{2}\left(p^{-} \ell^{+}\right)} \int_{0}^{\infty} \frac{d k^{-}}{k^{-}}\left[m_{1}^{2}\right]^{-\epsilon}\left[\frac{k^{-}\left(k^{-}-p^{-}\right)}{\mu_{-}{ }^{2}}\right]^{\epsilon} .
\end{aligned}
$$

The subtraction integral cancels the singularity in $\tilde{I}_{\mathrm{cn}}^{\text {scalar }}$ as $k^{-} \rightarrow 0$ as long as $m_{1} \neq 0$. The complete collinear result, $I_{\mathrm{cn}}^{\text {scalar }}=\tilde{I}_{\mathrm{cn}}^{\text {scalar }}-I_{0 \mathrm{cn}}^{\text {scalar }}$, is very similar to the soft result

$$
\begin{gathered}
I_{\mathrm{cn}}^{\mathrm{scalar}=}=\frac{-i \Gamma(\epsilon)}{16 \pi^{2}\left(p^{-} \ell^{+}\right)}\left(\frac{p^{-}}{\mu_{-}}\right)^{2 \epsilon}\left\{\int_{0}^{1} d x \frac{|1-x|^{\epsilon}|x|^{\epsilon}}{x}\left[\frac{m_{1}^{2}(1-x)+m_{3}^{2} x}{\mu^{2}}\right]^{-\epsilon}-\int_{0}^{\infty} d x \frac{|1-x|^{\epsilon}|x|^{\epsilon}}{x}\left[\frac{m_{1}^{2}}{\mu^{2}}\right]^{-\epsilon}\right\} \\
=\frac{-i}{16 \pi^{2}\left(p^{-} \ell^{+}\right)}\left[\frac{1}{2 \epsilon_{\mathrm{UV}}^{2}}+\frac{1}{\epsilon_{\mathrm{UV}}} \ln \left(\frac{p^{-}}{\mu_{-}}\right)-\frac{1}{2 \epsilon_{\mathrm{UV}}} \ln \left(\frac{m_{1}^{2}}{\mu^{2}}\right)+\ln ^{2}\left(\frac{p^{-}}{\mu_{-}}\right)+\frac{5 \pi^{2}}{24}\right. \\
\left.\quad+\frac{1}{4} \ln ^{2}\left(\frac{m_{1}^{2}}{\mu^{2}}\right)-\ln \left(\frac{m_{1}^{2}}{\mu^{2}}\right) \ln \left(\frac{p^{-}}{\mu_{-}}\right)+\operatorname{Li}_{2}\left(1-\frac{m_{3}^{2}}{m_{1}^{2}}\right)\right] .
\end{gathered}
$$

The results in Eqs. (B5) and (B7) have $1 / \epsilon_{\mathrm{UV}} \ln \left(m^{2}\right)$ divergences, which are canceled by the $\phi(0, \mu) / \epsilon$ type counterterms. For this scalar calculation these divergences are canceled by a graph containing the insertion 
of the renormalized currents in Eq. (112) with additional counterterm coefficients for the convolution integral as given in Eq. (113). Contracting the scalar gluon as in Fig. 15b), using the same IR mass regulator, and pulling out the same prefactor as the other diagrams gives

$$
I_{\mathrm{ct}}^{\text {scalar }}=2\left(\frac{1}{2 \epsilon_{\mathrm{UV}}}\right) \frac{i}{16 \pi^{2}\left(p^{-} \ell^{+}\right)}\left[-\ln \left(\frac{m_{1}^{2}}{\mu^{2}}\right)\right] .
$$

Note that dependence on $m_{2,3}$ drops out of the answer for $I_{\mathrm{ct}}^{\text {scalar }}$. Due to our choice of $\delta C^{(0 d, 0 e)}$ this exactly cancels the $1 / \epsilon_{\mathrm{UV}} \ln \left(m_{1}^{2}\right)$ terms in the collinear and soft loops. Adding the soft, collinear, and counterterm graphs we find the full $\mathrm{SCET}_{\mathrm{II}}$ result

$$
\begin{aligned}
I_{\mathrm{soft}+\mathrm{cn}}^{\mathrm{scalar}}= & \frac{-i}{16 \pi^{2}\left(p^{-} \ell^{+}\right)}\left[\frac{1}{2} \ln ^{2}\left(\frac{m_{1}^{2}}{\mu^{2}}\right)-\ln \left(\frac{m_{1}^{2}}{\mu^{2}}\right) \ln \left(\frac{p^{-}}{\mu_{-}}\right)-\ln \left(\frac{m_{1}^{2}}{\mu^{2}}\right) \ln \left(\frac{\ell^{+}}{\mu_{+}}\right)+\operatorname{Li}_{2}\left(1-\frac{m_{2}^{2}}{m_{1}^{2}}\right)+\operatorname{Li}_{2}\left(1-\frac{m_{3}^{2}}{m_{1}^{2}}\right)\right. \\
& \left.+\frac{1}{\epsilon_{\mathrm{UV}}^{2}}+\frac{1}{\epsilon_{\mathrm{UV}}} \ln \left(\frac{p^{-} \ell^{+}}{\mu_{-} \mu_{+}}\right)+\ln ^{2}\left(\frac{p^{-}}{\mu_{-}}\right)+\ln ^{2}\left(\frac{\ell^{+}}{\mu_{+}}\right)+\frac{5 \pi^{2}}{12}\right] \\
= & \frac{-i}{16 \pi^{2}\left(p^{-} \ell^{+}\right)}\left[\frac{1}{2} \ln ^{2}\left(\frac{m_{1}^{2}}{p^{-} \ell^{+}}\right)-\ln \left(\frac{m_{1}^{2}}{\mu^{2}}\right) \ln \left(\frac{\mu^{2}}{\mu_{-} \mu_{+}}\right)+\operatorname{Li}_{2}\left(1-\frac{m_{2}^{2}}{m_{1}^{2}}\right)+\operatorname{Li}_{2}\left(1-\frac{m_{3}^{2}}{m_{1}^{2}}\right)\right. \\
& \left.+\frac{1}{\epsilon_{\mathrm{UV}}^{2}}+\frac{1}{\epsilon_{\mathrm{UV}}} \ln \left(\frac{p^{-} \ell^{+}}{\mu_{-} \mu_{+}}\right)+\ln ^{2}\left(\frac{p^{-}}{\mu_{-}}\right)+\ln ^{2}\left(\frac{\ell^{+}}{\mu_{+}}\right)-\frac{1}{2} \ln ^{2}\left(\frac{p^{-} \ell^{+}}{\mu^{2}}\right)+\frac{5 \pi^{2}}{12}\right] .
\end{aligned}
$$

The effective theory result in Eq. (B9) has UV divergences which are the same as in Eq. (115), and are canceled by a counterterm for the jet function coefficient $J^{(0 a)}$, as given in Eq. (116). The renormalized EFT result is

$$
\begin{aligned}
I_{\text {soft }+\mathrm{cn}}^{\text {scalar }}=\frac{-i}{16 \pi^{2}\left(p^{-} \ell^{+}\right)} & {\left[\frac{1}{2} \ln ^{2}\left(\frac{m_{1}^{2}}{p^{-} \ell^{+}}\right)+\operatorname{Li}_{2}\left(1-\frac{m_{2}^{2}}{m_{1}^{2}}\right)+\operatorname{Li}_{2}\left(1-\frac{m_{3}^{2}}{m_{1}^{2}}\right)-\ln \left(\frac{m_{1}^{2}}{\mu^{2}}\right) \ln \left(\frac{\mu^{2}}{\mu_{-} \mu_{+}}\right)\right.} \\
+ & \left.\ln ^{2}\left(\frac{p^{-}}{\mu_{-}}\right)+\ln ^{2}\left(\frac{\ell^{+}}{\mu_{+}}\right)-\frac{1}{2} \ln ^{2}\left(\frac{p^{-} \ell^{+}}{\mu^{2}}\right)+\frac{5 \pi^{2}}{12}\right] .
\end{aligned}
$$

The first three terms exactly reproduces the IR divergences in the full theory result in Eq. (B1), including the entire functional dependence on the ratios of $m_{i}^{2}$, and the fourth term vanishes since $\mu^{2}=\mu_{+} \mu_{-}$. The difference of the remaining finite terms gives a contribution to the one-loop matching coefficient

$$
\begin{aligned}
I_{\text {match }}^{\text {scalar }} & =\frac{-i}{16 \pi^{2}\left(p^{-} \ell^{+}\right)}\left[\frac{1}{2} \ln ^{2}\left(\frac{p^{-} \ell^{+}}{\mu_{-} \mu_{+}}\right)-\ln ^{2}\left(\frac{p^{-}}{\mu_{-}}\right)-\ln ^{2}\left(\frac{\ell^{+}}{\mu_{+}}\right)-\frac{5 \pi^{2}}{12}\right] \\
& =\frac{-i}{16 \pi^{2}\left(p^{-} \ell^{+}\right)}\left[-\frac{1}{2} \ln ^{2}\left(\frac{p^{-} \mu_{+}}{\mu_{-} \ell^{+}}\right)-\frac{5 \pi^{2}}{12}\right] .
\end{aligned}
$$

This result exactly reproduces the matching coefficient in Eq. (119), as anticipated. In the limit $m_{2,3} \rightarrow 0$ all results go smoothly over to those in section VIIB1.

ii) Offshellness $\mathbf{p}^{2}=-\mathbf{P}^{2} \neq 0, \ell^{2}=-\mathbf{L}^{2} \neq 0$, with $\mathbf{m}_{1} \neq 0$ and $\mathbf{m}_{2,3}=0$

The full theory loop integral is now

$$
\begin{aligned}
I_{\text {full }}^{\text {scalar }} & =\int \frac{d^{D} k}{(2 \pi)^{D}} \frac{1}{\left[(k-\ell)^{2}+i 0^{+}\right]\left[k^{2}-m_{1}^{2}+i 0^{+}\right]\left[(k-p)^{2}+i 0^{+}\right]} \\
& =\frac{-i}{16 \pi^{2}\left(p^{-} \ell^{+}\right)}\left[\frac{1}{2} \ln ^{2}\left(\frac{m_{1}^{2}}{p^{-} \ell^{+}}\right)+\operatorname{Li}_{2}\left(\frac{-L^{2}}{m_{1}^{2}}\right)+\operatorname{Li}_{2}\left(\frac{-P^{2}}{m_{1}^{2}}\right)+\frac{\pi^{2}}{3}\right] .
\end{aligned}
$$

where $\ell^{2}=-L^{2}, p^{2}=-P^{2}$ and we have expanded in $P^{2} /\left(p^{-} \ell^{+}\right), L^{2} /\left(p^{-} \ell^{+}\right)$, and $m_{1}^{2} /\left(p^{-} \ell^{+}\right)$. The result is valid as long as $\left(p^{-} \ell^{+}\right) m_{1}^{2} \gg P^{2} L^{2}$, and so can not be used for the special case $m_{1}=0$. The result for $m_{1} \rightarrow 0$ is discussed below in case iii). 
Using the same IR regulators for the scalar and collinear SCET II loops in Figs. 15b,c we have

$$
\begin{aligned}
I_{\mathrm{soft}}^{\text {scalar }} & =\sum_{k^{+} \neq 0} \int \frac{d^{D} k_{r}}{(2 \pi)^{D}} \frac{\mu^{2 \epsilon}}{\left[(k-\ell)^{2}+i 0^{+}\right]\left[k^{2}-m_{1}^{2}+i 0^{+}\right]\left[-p^{-} k^{+}+i 0^{+}\right]} \frac{\left.\left|k^{+}\right|\right|^{+}\left|k^{+}-\ell^{+}\right|^{\epsilon}}{\mu_{+}^{2 \epsilon}}, \\
I_{\mathrm{cn}}^{\text {scalar }} & =\sum_{k^{-} \neq 0} \int \frac{d^{D} k_{r}^{\prime}}{(2 \pi)^{D}} \frac{\mu^{2 \epsilon}}{\left[-\ell^{+} k^{-}+i 0^{+}\right]\left[k^{2}-m_{1}^{2}+i 0^{+}\right]\left[(k-p)^{2}+i 0^{+}\right]} \frac{\left.\left|k^{-}\right|\right|^{\epsilon}\left|k^{-}-p^{-}\right|^{\epsilon}}{\mu_{-}^{2 \epsilon}} .
\end{aligned}
$$

Note that we keep $\ell^{2}$ and $p^{2}$ only in the propagators that are allowed to become small by the power counting in $\mathrm{SCET}_{\mathrm{II}}$. Eq. (17) tells us that we have zero-bin subtractions for the soft and collinear diagrams and the naive integrals and subtraction integrals are

$$
\begin{aligned}
\tilde{I}_{\mathrm{soft}}^{\text {scalar }} & =\int \frac{d^{D} k}{(2 \pi)^{D}} \frac{\mu^{2 \epsilon}}{\left[(k-\ell)^{2}+i 0^{+}\right]\left[k^{2}-m_{1}^{2}+i 0^{+}\right]\left[-p^{-} k^{+}+i 0^{+}\right]} \frac{\left|k^{+}\right|{ }^{\epsilon}\left|k^{+}-\ell^{+}\right|^{\epsilon}}{\mu_{+}^{2 \epsilon}}, \\
I_{0 \mathrm{soft}}^{\text {scalar }} & =\int \frac{d^{D} k}{(2 \pi)^{D}} \frac{\mu^{2 \epsilon}}{\left[-\ell^{+} k^{-}+i 0^{+}\right]\left[k^{2}-m_{1}^{2}+i 0^{+}\right]\left[-p^{-} k^{+}+i 0^{+}\right]} \frac{\left|k^{+}\right|{ }^{\epsilon}\left|k^{+}-\ell^{+}\right|^{\epsilon}}{\mu_{+}^{2 \epsilon}}, \\
\tilde{I}_{\mathrm{cn}}^{\text {scalar }} & =\int \frac{d^{D} k}{(2 \pi)^{D}} \frac{\mu^{2 \epsilon}}{\left[-\ell^{+} k^{-}+i 0^{+}\right]\left[k^{2}-m_{1}^{2}+i 0^{+}\right]\left[(k-p)^{2}+i 0^{+}\right]} \frac{k^{-}\left|k^{-}-p^{-}\right|^{\epsilon}}{\mu_{-}^{2 \epsilon}}, \\
I_{0 \mathrm{cn}}^{\text {scalar }} & =\int \frac{d^{D} k}{(2 \pi)^{D}} \frac{\left.\left|k^{-}\right|\right|^{\epsilon}\left|k^{-}-p^{-}\right|^{\epsilon}}{\mu_{-}^{2 \epsilon}} .
\end{aligned}
$$

To determine the form of the subtraction integrals, we considered the collinear limit of the soft loop momentum in $\tilde{I}_{\text {soft }}^{\text {scalar }}$, and the soft limit of the collinear loop momentum in $\tilde{I}_{\mathrm{cn}}^{\text {scalar }}$. The $p^{2}$ and $\ell^{2}$ dependence is dropped in the subtraction integrals because in these limits $\ell^{+} k^{-} \gg-\ell^{2}$ and $p^{-} k^{+} \gg-p^{2}$. Note that the subtraction integrals are identical to the case with $m_{1,2,3} \neq 0$. The final results $I_{\text {soft }}^{\text {scalar }}$ and $I_{\text {cn }}^{\text {scalar }}$ are defined by the differences $\tilde{I}_{\text {soft }}^{\text {scalar }}-I_{0 \text { soft }}^{\text {scalar }}$ and $\tilde{I}_{\text {cn }}^{\text {scalar }}-I_{0 \mathrm{cn}}^{\text {scalar }}$ respectively.

To compute the soft graph we work in the frame where $\ell^{\perp}=0$, thus $\ell^{+} \ell^{-}=-L^{2}<0$ with $\ell^{+}>0$, so the offshell momentum is $\ell^{-}=-L^{-}<0$. We do the $k^{-}$integral by contours. Due to the pole structure this restricts the $k^{+}$-integration to the region $0<k^{+}<\ell^{+}$. The $k_{\perp}$ integral is then done. For the soft subtraction integral we follow the same procedure which gives the integration region $0<k^{+}<\infty$. Thus

$$
\begin{aligned}
\tilde{I}_{\text {soft }}^{\text {scalar }} & =\frac{-i \Gamma(\epsilon) \mu^{2 \epsilon}}{16 \pi^{2}\left(p^{-} \ell^{+}\right)} \int_{0}^{\ell^{+}} \frac{d k^{+}}{k^{+}}\left[\left(1-\frac{k^{+}}{\ell^{+}}\right)\left(m_{1}^{2}+k^{+} L^{-}\right)\right]^{-\epsilon}\left|\frac{k^{+}\left(k^{+}-\ell^{+}\right)}{\mu_{+}{ }^{2}}\right|^{\epsilon}, \\
I_{0 \text { soft }}^{\text {scalar }} & =\frac{-i \Gamma(\epsilon) \mu^{2 \epsilon}}{16 \pi^{2}\left(p^{-} \ell^{+}\right)} \int_{0}^{\infty} \frac{d k^{+}}{k^{+}}\left[m_{1}^{2}\right]^{-\epsilon}\left|\frac{k^{+}\left(k^{+}-\ell^{+}\right)}{\mu_{+}{ }^{2}}\right|^{\epsilon} .
\end{aligned}
$$

The double counting with the collinear integral comes from the $k^{+} \rightarrow 0$ part of the integral, but the divergence from this limit exactly cancels in $I_{\text {soft }}^{\text {scalar }}=\tilde{I}_{\text {soft }}^{\text {scalar }}-I_{0 \text { soft }}^{\text {scalar }}$ as long as $m_{1} \neq 0$. Computing the integrals we find

$$
\begin{aligned}
I_{\mathrm{soft}}^{\mathrm{scalar}}= & \frac{-i \Gamma(\epsilon)}{16 \pi^{2}\left(p^{-} \ell^{+}\right)}\left(\frac{\ell^{+}}{\mu_{+}}\right)^{2 \epsilon}\left\{\int_{0}^{1} d x \frac{|1-x|^{\epsilon}|x|^{\epsilon}}{x}\left[\frac{(1-x)\left(m_{1}^{2}+x L^{2}\right)}{\mu^{2}}\right]^{-\epsilon}-\int_{0}^{\infty} d x \frac{|1-x|^{\epsilon}|x|^{\epsilon}}{x}\left[\frac{m_{1}^{2}}{\mu^{2}}\right]^{-\epsilon}\right\} \\
= & \frac{-i \Gamma(\epsilon)}{16 \pi^{2}\left(p^{-} \ell^{+}\right)}\left(\frac{\mu^{2}}{m_{1}^{2}}\right)^{\epsilon}\left(\frac{\ell^{+}}{\mu_{+}}\right)^{2 \epsilon}\left\{\frac{\Gamma(\epsilon)}{\Gamma(1+\epsilon)} F_{1}\left(\epsilon, \epsilon, 1+\epsilon, \frac{-L^{2}}{m_{1}^{2}}\right)-\frac{\Gamma(\epsilon) \Gamma(1+\epsilon)}{\Gamma(1+2 \epsilon)}-\frac{\Gamma(1+\epsilon) \Gamma(-2 \epsilon)}{\Gamma(1-\epsilon)}\right\} \\
= & \frac{-i}{16 \pi^{2}\left(p^{-} \ell^{+}\right)}\left[\frac{1}{2 \epsilon_{\mathrm{UV}}^{2}}+\frac{1}{\epsilon_{\mathrm{UV}}} \ln \left(\frac{\ell^{+}}{\mu_{+}}\right)-\frac{1}{2 \epsilon_{\mathrm{UV}}} \ln \left(\frac{m_{1}^{2}}{\mu^{2}}\right)+\ln ^{2}\left(\frac{\ell^{+}}{\mu_{+}}\right)+\frac{3 \pi^{2}}{8}\right. \\
& \left.+\frac{1}{4} \ln ^{2}\left(\frac{m_{1}^{2}}{\mu^{2}}\right)-\ln \left(\frac{m_{1}^{2}}{\mu^{2}}\right) \ln \left(\frac{\ell^{+}}{\mu_{+}}\right)+\operatorname{Li}_{2}\left(\frac{-L^{2}}{m_{1}^{2}}\right)\right] .
\end{aligned}
$$

For the collinear integrals we take $p^{\perp}=0$, so $p^{-} p^{+}=-P^{2}<0$ with $p^{-}>0$, and it is $p^{+}=-P^{+}<0$ that takes the $p^{2}$ offshell. We do the contour integration in $k^{+}$which restricts the remaining integration region 
in $k^{-}$. For the naive and subtraction integrals we find

$$
\begin{aligned}
\tilde{I}_{\mathrm{cn}}^{\text {scalar }} & =\frac{-i \Gamma(\epsilon) \mu^{2 \epsilon}}{16 \pi^{2}\left(p^{-} \ell^{+}\right)} \int_{0}^{p^{-}} \frac{d k^{-}}{k^{-}}\left[\left(1-\frac{k^{-}}{p^{-}}\right)\left(m_{1}^{2}+k^{-} P^{+}\right)\right]^{-\epsilon}\left|\frac{k^{-}\left(k^{-}-p^{-}\right)}{\mu_{-}{ }^{2}}\right|^{\epsilon} \\
I_{0 \mathrm{cn}}^{\text {scalar }} & =\frac{-i \Gamma(\epsilon) \mu^{2 \epsilon}}{16 \pi^{2}\left(p^{-} \ell^{+}\right)} \int_{0}^{\infty} \frac{d k^{-}}{k^{-}}\left[m_{1}^{2}\right]^{-\epsilon}\left[\frac{k^{-}\left(k^{-}-p^{-}\right)}{\mu_{-}{ }^{2}}\right]^{\epsilon}
\end{aligned}
$$

The complete collinear result, $I_{\mathrm{cn}}^{\text {scalar }}=\tilde{I}_{\mathrm{cn}}^{\text {scalar }}-I_{0 \mathrm{cn}}^{\text {scalar }}$, is very similar to the soft result

$$
\begin{gathered}
I_{\mathrm{cn}}^{\text {scalar }}=\frac{-i \Gamma(\epsilon)}{16 \pi^{2}\left(p^{-} \ell^{+}\right)}\left(\frac{p^{-}}{\mu_{-}}\right)^{2 \epsilon}\left\{\int_{0}^{1} d x \frac{|1-x|^{\epsilon}|x|^{\epsilon}}{x}\left[\frac{(1-x)\left(m_{1}^{2}+x P^{2}\right)}{\mu^{2}}\right]^{-\epsilon}-\int_{0}^{\infty} d x \frac{|1-x|^{\epsilon}|x|^{\epsilon}}{x}\left[\frac{m_{1}^{2}}{\mu^{2}}\right]^{-\epsilon}\right\} \\
=\frac{-i}{16 \pi^{2}\left(p^{-} \ell^{+}\right)}\left[\frac{1}{2 \epsilon_{\mathrm{UV}}^{2}}+\frac{1}{\epsilon_{\mathrm{UV}}} \ln \left(\frac{p^{-}}{\mu_{-}}\right)-\frac{1}{2 \epsilon_{\mathrm{UV}}} \ln \left(\frac{m_{1}^{2}}{\mu^{2}}\right)+\ln ^{2}\left(\frac{p^{-}}{\mu_{-}}\right)+\frac{3 \pi^{2}}{8}\right. \\
\left.\quad+\frac{1}{4} \ln ^{2}\left(\frac{m_{1}^{2}}{\mu^{2}}\right)-\ln \left(\frac{m_{1}^{2}}{\mu^{2}}\right) \ln \left(\frac{p^{-}}{\mu_{-}}\right)+\operatorname{Li}_{2}\left(\frac{-P^{2}}{m_{1}^{2}}\right)\right] .
\end{gathered}
$$

The results in Eqs. (B16) and (B18) have $1 / \epsilon_{\mathrm{UV}} \ln \left(m^{2}\right)$ divergences, which are canceled by the $\phi(0, \mu) / \epsilon$ type counterterms just as in our $m_{1,2,3}$ case. The counterterms and result to be added are the same as in Eq. (B8) and exactly cancels the $1 / \epsilon_{\mathrm{UV}} \ln \left(m^{2}\right)$ terms in the collinear and soft loops. Adding the soft, collinear, and counterterm graphs and simplifying we find the full SCET $_{\text {II }}$ result

$$
\begin{aligned}
I_{\mathrm{soft}+\mathrm{cn}}^{\text {scalar }}= & \frac{-i}{16 \pi^{2}\left(p^{-} \ell^{+}\right)}\left[\frac{1}{2} \ln ^{2}\left(\frac{m_{1}^{2}}{p^{-} \ell^{+}}\right)-\ln \left(\frac{m_{1}^{2}}{\mu^{2}}\right) \ln \left(\frac{\mu^{2}}{\mu_{-} \mu_{+}}\right)+\operatorname{Li}_{2}\left(\frac{-P^{2}}{m_{1}^{2}}\right)+\operatorname{Li}_{2}\left(\frac{-L^{2}}{m_{1}^{2}}\right)\right. \\
& \left.+\frac{1}{\epsilon_{\mathrm{UV}}^{2}}-\frac{1}{\epsilon_{\mathrm{UV}}}+\frac{1}{\epsilon_{\mathrm{UV}}} \ln \left(\frac{p^{-} \ell^{+}}{\mu_{-} \mu_{+}}\right)+\ln ^{2}\left(\frac{p^{-}}{\mu_{-}}\right)+\ln ^{2}\left(\frac{\ell^{+}}{\mu_{+}}\right)-\frac{1}{2} \ln ^{2}\left(\frac{p^{-} \ell^{+}}{\mu^{2}}\right)+\frac{3 \pi^{2}}{4}\right] .
\end{aligned}
$$

The effective theory result in Eq. (B19) has UV divergences which are canceled by the counterterm for the jet function coefficient $J^{(0 a)}$, as already given in Eq. (116). The renormalized EFT result is

$$
\begin{aligned}
I_{\text {soft }+ \text { cn }}^{\text {scalar }}=\frac{-i}{16 \pi^{2}\left(p^{-} \ell^{+}\right)} & {\left[\frac{1}{2} \ln ^{2}\left(\frac{m_{1}^{2}}{p^{-} \ell^{+}}\right)+\operatorname{Li}_{2}\left(\frac{-P^{2}}{m_{1}^{2}}\right)+\operatorname{Li}_{2}\left(\frac{-L^{2}}{m_{1}^{2}}\right)-\ln \left(\frac{m_{1}^{2}}{\mu^{2}}\right) \ln \left(\frac{\mu^{2}}{\mu_{-} \mu_{+}}\right)\right.} \\
+ & \left.\ln ^{2}\left(\frac{p^{-}}{\mu_{-}}\right)+\ln ^{2}\left(\frac{\ell^{+}}{\mu_{+}}\right)-\frac{1}{2} \ln ^{2}\left(\frac{p^{-} \ell^{+}}{\mu^{2}}\right)+\frac{3 \pi^{2}}{4}\right] .
\end{aligned}
$$

The first three terms exactly reproduces the IR divergences in the full theory result in Eq. (B12), including the entire functional dependence on $P^{2} / m_{1}^{2}$ and $L^{2} / m_{1}^{2}$. The fourth term vanishes since $\mu^{2}=\mu_{+} \mu_{-}$. The difference of the remaining finite terms gives a contribution to the one-loop matching coefficient

$$
\begin{aligned}
I_{\text {match }}^{\text {scalar }} & =\frac{-i}{16 \pi^{2}\left(p^{-} \ell^{+}\right)}\left[\frac{1}{2} \ln ^{2}\left(\frac{p^{-} \ell^{+}}{\mu_{-} \mu_{+}}\right)-\ln ^{2}\left(\frac{p^{-}}{\mu_{-}}\right)-\ln ^{2}\left(\frac{\ell^{+}}{\mu_{+}}\right)-\frac{5 \pi^{2}}{12}\right] \\
& =\frac{-i}{16 \pi^{2}\left(p^{-} \ell^{+}\right)}\left[-\frac{1}{2} \ln ^{2}\left(\frac{p^{-} \mu_{+}}{\mu_{-} \ell^{+}}\right)-\frac{5 \pi^{2}}{12}\right] .
\end{aligned}
$$

This result exactly reproduces the matching coefficient in Eqs. (119) and (B11) using a different IR regulator. This is as expected since the full theory was UV finite, and the same UV regulator was used in the SCET calculation. In the limit $L^{2}, P^{2} \rightarrow 0$ all the results go smoothly over to the results presented in section VII B 1 .

\section{iii) The limit $\mathrm{m}_{1} \rightarrow 0$ of cases i) and ii)}

Finally, we discuss the limit $m_{1} \rightarrow 0$ of the IR regulators considered above in cases i) and ii). This is not a smooth limit in either the full or effective theories. In the following we use the notation $Q^{2} \equiv\left(p^{-} \ell^{+}\right)$ as a shorthand for our large perturbative scale. 
We first consider the full theory loop integrals, but in expanding out the IR regulators we keep the first subleading terms in the expansions in cases where the leading term vanishes as $m_{1}^{2} \rightarrow 0$. So for the expansion in $m_{1,2,3}^{2} / Q^{2}$ in case i), we keep subleading $m_{2,3}^{2}$ terms if the leading term is proportional to $m_{1}^{2}$. For the expansion in $m_{1}^{2} / Q^{2}, L^{2} / Q^{2}$, and $P^{2} / Q^{2}$ in case ii) we keep subleading $L^{2}$ and $P^{2}$ terms when the leading term is proportional to $m_{1}^{2}$. For the $m_{1,2,3} \neq 0$ regulator this gives

$$
\begin{aligned}
& I_{\text {full }}^{\text {scalar }}=\frac{-i}{16 \pi^{2}\left(p^{-} \ell^{+}\right)}\{\left\{\frac{1}{2} \ln ^{2}\left[\frac{\xi-i 0^{+}}{Q^{4}}\right]+\operatorname{Li}_{2}\left[\frac{Q^{2}\left(m_{1}^{2}-m_{2}^{2}\right)}{\xi}-i 0^{+}\right]\right. \\
&\left.+\operatorname{Li}_{2}\left[\frac{Q^{2}\left(m_{1}^{2}-m_{3}^{2}\right)}{\xi}-i 0^{+}\right]-\operatorname{Li}_{2}\left[\frac{-\left(m_{1}^{2}-m_{2}^{2}\right)\left(m_{1}^{2}-m_{3}^{2}\right)}{\xi}\right]\right\}, \\
& \xi \equiv Q^{2} m_{1}^{2}-m_{2}^{2} m_{3}^{2},
\end{aligned}
$$

while for the $m_{1}^{2} \neq 0, p^{2}=-P^{2} \neq 0$, and $\ell^{2}=-L^{2} \neq 0$ we have

$$
\begin{aligned}
I_{\text {full }}^{\text {scalar }} & =\frac{-i}{16 \pi^{2}\left(p^{-} \ell^{+}\right)}\left\{\frac{1}{2} \ln ^{2}\left[\frac{\xi-i 0^{+}}{Q^{4}}\right]+\operatorname{Li}_{2}\left[\frac{Q^{2} P^{2}}{-\xi+i 0^{+}}\right]+\operatorname{Li}_{2}\left[\frac{Q^{2} L^{2}}{-\xi+i 0^{+}}\right]+\frac{\pi^{2}}{3}-\operatorname{Li}_{2}\left[\frac{L^{2} P^{2}}{-\xi+i 0^{+}}\right]\right\}, \\
\xi & \equiv Q^{2} m_{1}^{2}-L^{2} P^{2} .
\end{aligned}
$$

From Eq. (B22) we see that as long as $Q^{2} m_{1}^{2} \gg m_{2}^{2} m_{3}^{2}$, as is the case if all the IR masses are the same order in the power counting, then $\xi \rightarrow Q^{2} m_{1}^{2}$, and expanding Eq. (B22) reproduces the result quoted in Eq. (B1). If we set $m_{1}=0$, then the leading $m_{i}^{2} / Q^{2}$ terms in the double log and di-logs vanish, and subleading terms regulate the IR divergences. In this case $\xi \rightarrow-m_{2}^{2} m_{3}^{2}$, and we obtain

$$
I_{\text {full }}^{\text {scalar }}\left(m_{1}=0\right)=\frac{-i}{16 \pi^{2}\left(p^{-} \ell^{+}\right)}\left[\ln \left(\frac{m_{2}^{2}}{Q^{2}}\right) \ln \left(\frac{m_{3}^{2}}{Q^{2}}\right)\right],
$$

We have checked that Eq. (B24) agrees with the result obtained by setting $m_{1}=0$ before evaluating the integral. Since subleading terms are regulating the IR divergences the result depends on the product $\ln \left(m_{2}^{2}\right) \ln \left(m_{3}^{2}\right)$ and thus no longer has a form that can be factorized in a straightforward manner into soft and collinear parts. The situation is very similar for case ii). Expanding Eq. (B23) when $Q^{2} m_{1}^{2} \gg L^{2} P^{2}$ gives $\xi \rightarrow Q^{2} m_{1}^{2}$ and reproduces the result quoted in Eq. (B12). If we set $m_{1}=0$ then the leading term in $\xi$ vanishes and subleading $L^{2}$ and $P^{2}$ terms regulate divergences in the double log and di-logs, with $\xi \rightarrow-L^{2} P^{2}$. In this case

$$
I_{\text {full }}^{\text {scalar }}\left(m_{1}=0\right)=\frac{-i}{16 \pi^{2}\left(p^{-} \ell^{+}\right)}\left[\ln \left(\frac{L^{2}}{Q^{2}}\right) \ln \left(\frac{P^{2}}{Q^{2}}\right)+\frac{\pi^{2}}{3}\right],
$$

To see why this happens we can examine the IR divergences in the full theory integral. Divergences occur for $k \rightarrow p^{-}, k \rightarrow \ell^{+}$, and $k \rightarrow 0$, and the issue with $m_{1} \rightarrow 0$ arises from the $k \rightarrow 0$ case where the propagators carrying the soft and collinear momenta are both singular in opposite light-like directions. The $k \rightarrow 0$ limit of the denominator of the integrand in Eq. (B1) is $\left[-\ell^{+} k^{-}-m_{2}^{2}\right]\left[k^{2}-m_{1}^{2}\right]\left[-p^{-} k^{+}-m_{3}^{2}\right]$ and for Eq. (B12) is $\left[-\ell^{+} k^{-}-L^{2}\right]\left[k^{2}-m_{1}^{2}\right]\left[-p^{-} k^{+}-P^{2}\right]$. The effect of the IR regulators is pictured by the solid curves in Fig. 17, where we show how they shield the integrand from blowing up when we go towards the $k^{-}=0$ or $k^{+}=0$ lines. Without the $m_{1}$ regulator the intersection of the $k^{+}=m_{3}^{2} / p^{-}$and $k^{-}=m_{2}^{2} / \ell^{+}$lines generates unphysical sensitivity to a very small scale $\sim m_{2}^{2} m_{3}^{2} / Q^{2}$. For the case with offshellness it is the intersection of the lines $k^{+}=P^{2} / p^{-}$and $k^{-}=L^{2} / \ell^{+}$generating sensitivity to the very small scale $\sim L^{2} P^{2} / Q^{2}$. The sensitivity to this new small scale was first pointed out in Ref. [75] where the IR regulator $L^{2} \neq 0, P^{2} \neq 0$ was used. The result in Eq. (B25) agrees with the one studied there. In Ref. 775 messenger modes with very small invariant mass were added to the effective theory to account for the dependence on this "messenger scale". However, in QCD the sensitivity to this small messenger scale 


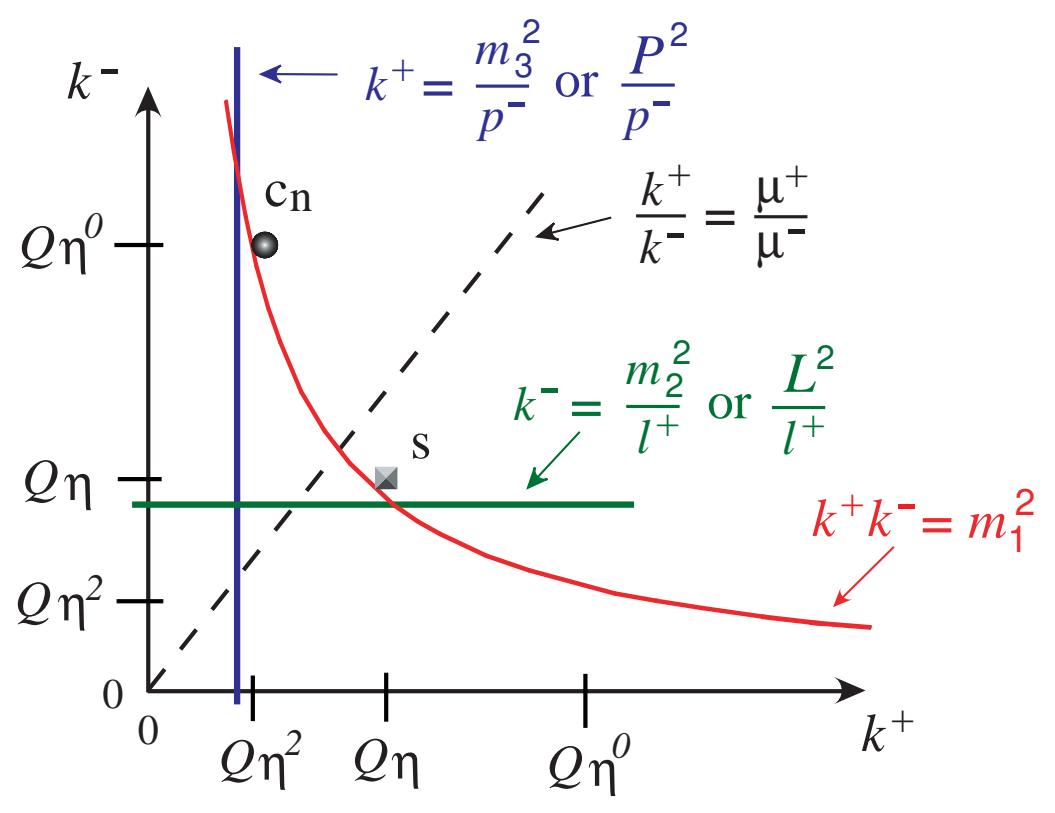

FIG. 17: Regulation of IR divergences in $\mathrm{SCET}_{\mathrm{II}}$ in perturbation theory in the $k^{+}-k^{-}$plane. The $c_{n}$ and $s$ denote the collinear and soft modes respectively, and the dashed $k^{+} / k^{-}=\mu^{+} / \mu^{-}$ line indicates how these modes are distinguished in perturbation theory. For $k^{\mu} \rightarrow 0$ the solid lines indicate at what scales the IR divergences are cutoff by the $m_{1}^{2}, m_{2}^{2}$, and $m_{3}^{2}$ regulators (and also for the $m_{1}^{2}, L^{2}, P^{2}$ regulator choice). Since $p^{-} \gg m_{i}$ the intersection of the $k^{+}=m_{3}^{2} / p^{-}$ and $k^{-}=m_{2}^{2} / \ell^{+}$lines is always below the curve $k^{+} k^{-}=m_{1}^{2}$ (and same for $k^{+}=P^{2} / p^{-}$and $\left.k^{-}=L^{2} / \ell^{+}\right)$. This intersection occurs at the messenger scale.

is unphysical because IR divergences are cutoff at an earlier stage by $\Lambda_{\mathrm{QCD}}$ 85. In perturbation theory with $m_{1} \neq 0$, sensitivity to the messenger scale also never appears. Comparing Fig. 13 with Fig. 17 we see that the $m_{1}^{2} \neq 0$ regulator behaves in a similar manner to $\Lambda_{\mathrm{QCD}}^{2}$. Other IR regulators are also known which remove the unphysical sensitivity to the messenger scale, including analytic regulators [78], and an energy dependent gluon mass 65 .

It is possible to choose IR regulators that complicate the choice of the matching coefficient; $m_{1}=0$ is such a choice. We have seen the complication in the full theory result in Eq. (B22). In the effective theory the choice $m_{1}=0$ also causes problems for $\tilde{I}_{\text {soft }}^{\text {scalar }}-I_{0 \text { soft }}^{\text {scalar }}$ and $\tilde{I}_{\mathrm{cn}}^{\text {scalar }}-I_{0 \mathrm{cn}}^{\text {scalar }}$. In this situation the $k_{\perp}$ integration for the subtraction integral is scaleless, and the subtraction terms do not cancel the problematic $k^{+} \rightarrow 0$ and $p^{-} \rightarrow 0$ regions in the naive integrals. For example the naive and subtraction integrals in Eq. (B4) scale with a different power of $k^{+}$as $k^{+} \rightarrow 0$. The same is true in for Eq. (B15), and for the collinear integrals. If one chooses $m_{1}=0$ some of the IR divergences are regulated at the scale $m_{2}^{2} m_{3}^{2} / Q^{2}$ or $L^{2} P^{2} / Q^{2}$, and so part of the IR regulator is of subleading order in the power counting with our definitions of the soft and collinear modes. This makes it difficult to compute the IR behavior in the effective theory, since one has to sum up a class of subleading terms in the power counting to all orders. We expect a resummation procedure in $\mathrm{SCET}_{\mathrm{II}}$ could be developed to reproduce the matching result in Eq. (119) with $m_{1}=0$ for the IR regulators considered in i) and ii), but we will not attempt it here.

It is worth emphasizing that the matching coefficient, Eqs. (119, B11), which depends on the difference between the full and effective theory results, does not depend on the IR regulator, as we have demonstrated with several different calculations. The matching coefficient is only sensitive to ultraviolet physics. It can be computed by using an IR regulator that is homogeneous in the power counting. We have seen this 
explicitly by computing the matching for arbitrary $m_{1,2,3}$, and for $P^{2}, L^{2}, m_{1}^{2}$ with $m_{1} \neq 0$. The purpose of the perturbation theory Feynman graph computations in the full and effective theories is to compute the matching coefficient Eq. (B4), which is a short distance quantity. The effective theory is not being used to reproduce the IR behavior of the full theory in perturbation theory for arbitrary IR regulators, especially regulators that only become effective at a small scale that is subleading order in the power counting. The

effective theory is being applied to QCD, where the infrared dynamics is nonperturbative, and cutoff at $\Lambda_{\mathrm{QCD}}$. 
[1] C. W. Bauer, S. Fleming, and M. E. Luke, Phys. Rev. D63, 014006 (2001), hep-ph/0005275.

[2] C. W. Bauer, S. Fleming, D. Pirjol, and I. W. Stewart, Phys. Rev. D63, 114020 (2001), hep-ph/0011336.

[3] C. W. Bauer and I. W. Stewart, Phys. Lett. B516, 134 (2001), hep-ph/0107001.

[4] C. W. Bauer, D. Pirjol, and I. W. Stewart, Phys. Rev. D65, 054022 (2002), hep-ph/0109045.

[5] W. E. Caswell and G. P. Lepage, Phys. Lett. B167, 437 (1986).

[6] G. T. Bodwin, E. Braaten, and G. P. Lepage, Phys. Rev. D51, 1125 (1995), hep-ph/9407339.

[7] N. Brambilla, A. Pineda, J. Soto, and A. Vairo, Nucl. Phys. B566, 275 (2000), hep$\mathrm{ph} / 9907240$.

[8] M. E. Luke, A. V. Manohar, and I. Z. Rothstein, Phys. Rev. D61, 074025 (2000), hep$\mathrm{ph} / 9910209$.

[9] A. V. Manohar and I. W. Stewart, Phys. Rev. D62, 074015 (2000), hep-ph/0003032.

[10] A. H. Hoang (2002), hep-ph/0204299.

[11] H. W. Griesshammer (1998), hep-ph/9804251.

[12] N. Brambilla, A. Pineda, J. Soto, and A. Vairo (2004), hep-ph/0410047.

[13] M. Beneke and V. A. Smirnov, Nucl. Phys. B522, 321 (1998), hep-ph/9711391.

[14] A. V. Manohar and I. W. Stewart, Phys. Rev. D63, 054004 (2001), hep-ph/0003107.

[15] A. H. Hoang, A. V. Manohar, and I. W. Stewart, Phys. Rev. D64, 014033 (2001), hep$\mathrm{ph} / 0102257$.

[16] S. Fleming and A. K. Leibovich, Phys. Rev. D67, 074035 (2003), hep-ph/0212094.

[17] C. W. Bauer and A. V. Manohar (2003), hep-ph/0312109.

[18] S. Fleming, A. K. Leibovich, and T. Mehen, Phys. Rev. D68, 094011 (2003), hep-ph/0306139.

[19] A. V. Manohar, Phys. Rev. D68, 114019 (2003), hep-ph/0309176.

[20] S. W. Bosch, B. O. Lange, M. Neubert, and G. Paz (2004), hep-ph/0402094.

[21] R. J. Hill, T. Becher, S. J. Lee, and M. Neubert (2004), hep-ph/0404217.

[22] B. V. Geshkenbein and M. V. Terentev, Phys. Lett. B117, 243 (1982).

[23] V. L. Chernyak and A. R. Zhitnitsky, Phys. Rept. 112, 173 (1984).

[24] R. Akhoury, G. Sterman, and Y. P. Yao, Phys. Rev. D50, 358 (1994).

[25] A. Szczepaniak, E. M. Henley, and S. J. Brodsky, Phys. Lett. B243, 287 (1990).

[26] A. V. Belitsky, X.-d. Ji, and F. Yuan, Phys. Rev. Lett. 91, 092003 (2003).

[27] S. Mantry, D. Pirjol, and I. W. Stewart, Phys. Rev. D68, 114009 (2003), hep-ph/0306254.

[28] M. Beneke, G. Buchalla, M. Neubert, and C. T. Sachrajda, Nucl. Phys. B606, 245 (2001).

[29] Y. Y. Keum, H.-N. Li, and A. I. Sanda, Phys. Rev. D63, 054008 (2001), hep-ph/0004173.

[30] Y.-Y. Keum, H.-n. Li, and A. I. Sanda, Phys. Lett. B504, 6 (2001), hep-ph/0004004.

[31] J. C. Collins, Acta Phys. Polon. B34, 3103 (2003).

[32] X.-d. Ji, J.-p. Ma, and F. Yuan, Phys. Rev. D71, 034005 (2005).

[33] J. C. Collins and D. E. Soper, Nucl. Phys. B193, 381 (1981).

[34] J. C. Collins and D. E. Soper, Nucl. Phys. B194, 445 (1982).

[35] C. W. Bauer, D. Pirjol, and I. W. Stewart, Phys. Rev. D67, 071502 (2003), hep-ph/0211069.

[36] G. Bell and T. Feldmann (2005), hep-ph/0509347.

[37] G. Sterman (2004), hep-ph/0412013.

[38] E. . Mueller, A. H. (1989), singapore: World Scientific (1989) 614 p. (Advanced series on directions in high energy physics, 5). Articles by Collins, Soper, Sterman and by Brodsky 
and Lepage.

[39] A. V. Manohar and M. B. Wise, Cambridge Monogr. Part. Phys. Nucl. Phys. Cosmol. 10, 1 (2000).

[40] A. V. Manohar (1996), hep-ph/9606222.

[41] A. V. Manohar, Phys. Rev. D56, 230 (1997), hep-ph/9701294.

[42] A. V. Manohar and I. W. Stewart, Phys. Rev. D62, 014033 (2000), hep-ph/9912226.

[43] A. V. Manohar and I. W. Stewart, Phys. Rev. Lett. 85, 2248 (2000), hep-ph/0004018.

[44] A. H. Hoang, Phys. Rev. D69, 034009 (2004), hep-ph/0307376.

[45] A. H. Hoang and P. Ruiz-Femenia, Phys. Rev. D73, 014015 (2006), hep-ph/0511102.

[46] A. Pineda and A. Vairo, Phys. Rev. D63, 054007 (2001), hep-ph/0009145.

[47] A. Pineda, Phys. Rev. D65, 074007 (2002), hep-ph/0109117.

[48] A. Pineda, Phys. Rev. D66, 054022 (2002), hep-ph/0110216.

[49] A. A. Penin, A. Pineda, V. A. Smirnov, and M. Steinhauser, Nucl. Phys. B699, 183 (2004), hep-ph/0406175.

[50] R. J. Hill and M. Neubert, Nucl. Phys. B657, 229 (2003), hep-ph/0211018.

[51] A. V. Manohar, J. Soto, and I. W. Stewart, Phys. Lett. B486, 400 (2000), hep-ph/0006096.

[52] A. H. Hoang, A. V. Manohar, I. W. Stewart, and T. Teubner, Phys. Rev. D65, 014014 (2002), hep-ph/0107144.

[53] A. H. Hoang and I. W. Stewart, Phys. Rev. D67, 114020 (2003), hep-ph/0209340.

[54] M. E. Luke and M. J. Savage, Phys. Rev. D57, 413 (1998), hep-ph/9707313.

[55] H. W. Griesshammer, Phys. Rev. D58, 094027 (1998), hep-ph/9712467.

[56] A. Pineda and J. Soto, Nucl. Phys. Proc. Suppl. 64, 428 (1998), hep-ph/9707481.

[57] H. Georgi, Phys. Lett. B240, 447 (1990).

[58] M. E. Luke and A. V. Manohar, Phys. Lett. B286, 348 (1992).

[59] B. Grinstein and I. Z. Rothstein, Phys. Rev. D57, 78 (1998), hep-ph/9703298.

[60] B. A. Kniehl, A. A. Penin, Y. Schroder, V. A. Smirnov, and M. Steinhauser, Phys. Lett. B607, 96 (2005), hep-ph/0412083.

[61] Y. Schroder, Phys. Lett. B447, 321 (1999), hep-ph/9812205.

[62] M. Peter, Nucl. Phys. B501, 471 (1997), hep-ph/9702245.

[63] T. Appelquist, M. Dine, and I. J. Muzinich, Phys. Lett. B69, 231 (1977).

[64] N. Brambilla, A. Pineda, J. Soto, and A. Vairo, Phys. Rev. D60, 091502 (1999), hep$\mathrm{ph} / 9903355$.

[65] C. W. Bauer, M. P. Dorsten, and M. P. Salem, Phys. Rev. D69, 114011 (2004).

[66] C. W. Bauer, S. Fleming, D. Pirjol, I. Z. Rothstein, and I. W. Stewart, Phys. Rev. D66, 014017 (2002), hep-ph/0202088.

[67] J. C. Collins, D. E. Soper, and G. Sterman, Nucl. Phys. B223, 381 (1983).

[68] C. W. Bauer, A. V. Manohar, and M. B. Wise, Phys. Rev. Lett. 91, 122001 (2003), hep$\mathrm{ph} / 0212255$.

[69] C. W. Bauer, C. Lee, A. V. Manohar, and M. B. Wise, Phys. Rev. D70, 034014 (2004), hep-ph/0309278.

[70] C. Lee and G. Sterman (2006), hep-ph/0603066.

[71] M. Beneke, A. P. Chapovsky, M. Diehl, and T. Feldmann, Nucl. Phys. B643, 431 (2002), hep-ph/0206152.

[72] G. Sterman (1995), hep-ph/9606312.

[73] R. K. Ellis, H. Georgi, M. Machacek, H. D. Politzer, and G. G. Ross, Nucl. Phys. B152, 285 (1979). 
[74] J. C. Collins, D. E. Soper, and G. Sterman, Adv. Ser. Direct. High Energy Phys. 5, 1 (1988), hep-ph/0409313.

[75] T. Becher, R. J. Hill, and M. Neubert, Phys. Rev. D69, 054017 (2004), hep-ph/0308122.

[76] G. Sterman (1993), cambridge, UK: Univ. Pr. (1993) 572 p.

[77] T. Becher, R. J. Hill, B. O. Lange, and M. Neubert, Phys. Rev. D69, 034013 (2004), hepph/0309227.

[78] M. Beneke and T. Feldmann, Nucl. Phys. B685, 249 (2004), hep-ph/0311335.

[79] A. V. Manohar, T. Mehen, D. Pirjol, and I. W. Stewart, Phys. Lett. B539, 59 (2002), hep-ph/0204229.

[80] B. O. Lange and M. Neubert, Nucl. Phys. B690, 249 (2004), hep-ph/0311345.

[81] G. P. Korchemsky, D. Pirjol, and T.-M. Yan, Phys. Rev. D61, 114510 (2000).

[82] E. Lunghi, D. Pirjol, and D. Wyler, Nucl. Phys. B649, 349 (2003).

[83] S. W. Bosch, R. J. Hill, B. O. Lange, and M. Neubert, Phys. Rev. D67, 094014 (2003).

[84] S. Descotes-Genon and C. T. Sachrajda, Phys. Lett. B557, 213 (2003).

[85] A. V. Manohar, Phys. Lett. B633, 729 (2006), hep-ph/0512173.

[86] I. Z. Rothstein, private communication (2005).

[87] T. Becher, R. J. Hill, and M. Neubert (2005), hep-ph/0503263.

[88] R. J. Hill, Phys. Rev. D73, 014012 (2006), hep-ph/0505129.

[89] V. A. Smirnov and E. R. Rakhmetov, Theor. Math. Phys. 120, 870 (1999), hep-ph/9812529.

[90] V. A. Smirnov, Springer Tracts Mod. Phys. 177, 1 (2002).

[91] B. O. Lange, A. V. Manohar, and I. W. Stewart (in preparation.).

[92] G. P. Lepage and S. J. Brodsky, Phys. Rev. D22, 2157 (1980).

[93] A. Hardmeier, E. Lunghi, D. Pirjol, and D. Wyler, Nucl. Phys. B682, 150 (2004).

[94] G. Burdman and J. F. Donoghue, Phys. Lett. B280, 287 (1992).

[95] M. B. Wise, Phys. Rev. D45, 2188 (1992).

[96] E. Gulez et al. (2006), hep-lat/0601021.

[97] M. Okamoto et al., Nucl. Phys. Proc. Suppl. 140, 461 (2005), hep-lat/0409116.

[98] G. Burdman and J. F. Donoghue, Phys. Lett. B270, 55 (1991).

[99] J. Charles, A. Le Yaouanc, L. Oliver, O. Pene, and J. C. Raynal, Phys. Rev. D60, 014001 (1999), hep-ph/9812358.

[100] M. J. Dugan and B. Grinstein, Phys. Lett. B255, 583 (1991).

[101] V. M. Belyaev, A. Khodjamirian, and R. Ruckl, Z. Phys. C60, 349 (1993), hep-ph/9305348.

[102] A. Khodjamirian, R. Ruckl, S. Weinzierl, and O. I. Yakovlev, Phys. Lett. B410, 275 (1997), hep-ph/9706303.

[103] E. Bagan, P. Ball, and V. M. Braun, Phys. Lett. B417, 154 (1998), hep-ph/9709243.

[104] M. Beneke and T. Feldmann, Nucl. Phys. B592, 3 (2001), hep-ph/0008255.

[105] M. Beneke, G. Buchalla, M. Neubert, and C. T. Sachrajda, Phys. Rev. Lett. 83, 1914 (1999), hep-ph/9905312.

[106] H. D. Politzer and M. B. Wise, Phys. Lett. B257, 399 (1991).

[107] C. W. Bauer, D. Pirjol, and I. W. Stewart, Phys. Rev. Lett. 87, 201806 (2001), hepph/0107002.

[108] T. Kurimoto, H.-n. Li, and A. I. Sanda, Phys. Rev. D65, 014007 (2002), hep-ph/0105003.

[109] D. Pirjol and I. W. Stewart, Phys. Rev. D67, 094005 (2003), hep-ph/0211251.

[110] C. W. Bauer, D. Pirjol, I. Z. Rothstein, and I. W. Stewart (2004), hep-ph/0401188.

[111] J. Chay and C. Kim, Nucl. Phys. B680, 302 (2004), hep-ph/0301262.

[112] A. G. Grozin and M. Neubert, Phys. Rev. D55, 272 (1997), hep-ph/9607366. 
[113] B. O. Lange (2004), hep-ph/0409277.

[114] H. Kawamura, J. Kodaira, C.-F. Qiao, and K. Tanaka, Phys. Lett. B523, 111 (2001), hep$\mathrm{ph} / 0109181$.

[115] M. Beneke, Y. Kiyo, and D. s. Yang (2004), hep-ph/0402241.

[116] T. Becher and R. J. Hill (2004), hep-ph/0408344.

[117] M. Beneke, G. Buchalla, M. Neubert, and C. T. Sachrajda (2000), hep-ph/0007256.

[118] T. D. Cohen, Phys. Rev. C66, 064003 (2002), nucl-th/0209072.

[119] C. W. Bauer and M. D. Schwartz (2006), hep-ph/0604065.

[120] J. C. Collins and F. Hautmann, JHEP 03, 016 (2001).

[121] M. Beneke and T. Feldmann, Phys. Lett. B553, 267 (2003), hep-ph/0211358. 